Florida International University

FIU Digital Commons

FIU Electronic Theses and Dissertations

University Graduate School

6-28-2019

\title{
Toward a fast and accurate modeling strategy for thermal management in air-cooled data centers
}

\author{
Long Tran Bao Phan \\ Florida International University, Iphan005@fiu.edu
}

Follow this and additional works at: https://digitalcommons.fiu.edu/etd

Part of the Computational Engineering Commons, Computer-Aided Engineering and Design

Commons, Energy Systems Commons, Heat Transfer, Combustion Commons, and the Other Mechanical

Engineering Commons

\section{Recommended Citation}

Phan, Long Tran Bao, "Toward a fast and accurate modeling strategy for thermal management in aircooled data centers" (2019). FIU Electronic Theses and Dissertations. 4243.

https://digitalcommons.fiu.edu/etd/4243

This work is brought to you for free and open access by the University Graduate School at FIU Digital Commons. It has been accepted for inclusion in FIU Electronic Theses and Dissertations by an authorized administrator of FIU Digital Commons. For more information, please contact dcc@fiu.edu. 


\section{FLORIDA INTERNATIONAL UNIVERSITY}

Miami, Florida

\section{TOWARD A FAST AND ACCURATE MODELING STRATEGY FOR THERMAL MANAGEMENT IN AIR-COOLED DATA CENTERS}

A dissertation submitted in partial fulfillment of

the requirements for the degree of

DOCTOR OF PHILOSOPHY

in

\section{MECHANICAL ENGINEERING}

by

Long Tran Bao Phan 
To: Dean John L. Volakis

College of Engineering and Computing

This dissertation, written by Long Tran Bao Phan, and entitled Toward a Fast and Accurate Modeling Strategy for Thermal Management in Air-Cooled Data Centers, having been approved in respect to style and intellectual content, is referred to you for judgment.

We have read this dissertation and recommend that it be approved.

Xiaobing Liu

Shaolei Ren

Arindam Chowdhury

Yiding Cao

George Stevo Dulikravich

Cheng-Xian Lin, Major Professor

Date of Defense: June 28, 2019

The dissertation of Long Tran Bao Phan is approved.

Dean John L. Volakis College of Engineering and Computing

Andrés G. Gil Vice President for Research and Economic Development and Dean of the University Graduate School

Florida International University, 2019 
(C) Copyright 2019 by Long Tran Bao Phan

All rights reserved. 


\section{DEDICATION}

Dedication of this work is given to my parents. Without their patience, understanding, support, and most of all love, the completion of this work would not have been possible. 


\section{ACKNOWLEDGMENTS}

I hereby would like to express my gratitude and appreciation to my major professor Dr. Cheng-Xian Lin who is kind and generous in supporting me every step of the way since I was an undergrad at the University of Tennessee in Knoxville, Tennessee. Dr. Lin not only advised me academically but also provided me many beautiful advices, encouragements, and continuous financial support in difficult time. I cannot thank Dr. Lin enough for providing an opportunity to do research with him.

I would to give thanks to my parents for the unconditional love and life-time support especially my mother who courageously let me be independent on my own feet and always have strong belief in me.

I would like to thank all of my committee members, especially, Professor Dulikravich and Professor Cao for their wonderful assistance in many useful graduate courses for my research, and your guidance for one of my research topics.

I am also thankful for the support of all the staff at the FIU graduate school and Mechanical \& Materials Department in various forms of support including graduate assistantship, scholarship, and fellowships.

I really appreciate the help and support of all my colleagues, especially, Soheil Soleimanikutanaei and Beichao Hu.

Lastly, I would like to acknowledge the generous optimization software support from Professor Carlo Poloni, Founder of ESTECO s.p.a. 
ABSTRACT OF THE DISSERTATION

TOWARD A FAST AND ACCURATE MODELING STRATEGY FOR THERMAL MANAGEMENT IN AIR-COOLED DATA CENTERS

by

\title{
Long Tran Bao Phan.
}

Florida International University, 2019

\author{
Miami, Florida \\ Professor Cheng-Xian Lin, Major Professor
}

Computational fluid dynamics (CFD) has become a popular tool compared to experimental measurement for thermal management in data centers. However, it is very time-consuming and resource-intensive when used to model large-scale data centers and may not be ready for real-time thermal monitoring. In this dissertation, the two main goals are first to develop rapid flow simulation methods to reduce the computing time while maintaining good accuracy, and second, to develop a whole building energy simulation (BES) strategy for data center modeling. To achieve this end, hybrid modeling and model training approaches are employed for rapid flow simulation, and a multi-zone model is proposed for BES. In the scope of hybrid modeling, two methods are proposed, i.e., the hybrid zero/two-equation turbulence model utilizing the zone partitioning technique and a combination of turbulence and floor tile models for the development of the composite performance index. It shows that the zero-equation coupled with either body force and modified body force tile models have the best potential in reducing the computing time, while preserving reasonable accuracy. The hybrid zero/two-equation method cuts down the computing time in half compared to the traditional practice of using only the two-equation model. In the scope of 
model training based approach, the reduced order method via proper orthogonal decomposition (POD) and response surface methodology (RSM) are comprehensively studied for data center modeling. Both methods can quickly reconstruct the data center thermal profile and retain good accuracy. The RSM method especially shows numerous advantages in several optimization studies of data centers. Whether it is for the tile selection to control the server rack temperature difference or impacting the decision for the input design parameters in the early stage of data center infrastructure design, RSM can replace costly experiments and time-consuming and resource-intensive CFD simulations. Finally, for the whole BES study, the proposed multi-zone model is found to be much more effective compared to the commonly used single zone model. The location factor plays an important role in deciding whether some of boundary conditions are affecting the cooling electricity consumption. In addition, the effect of supply temperature and volumetric flow rate have significant effects on the energy consumption. 


\section{TABLE OF CONTENTS}

CHAPTER

PAGE

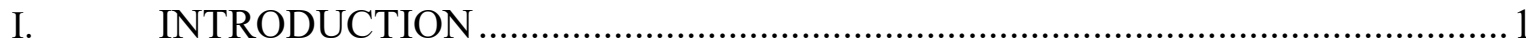

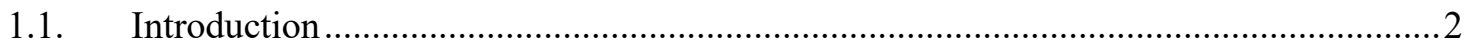

1.2. Background and Problem Definition .........................................................................

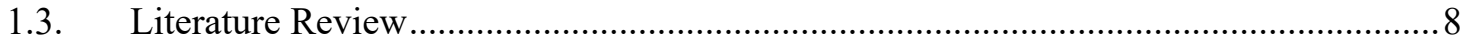

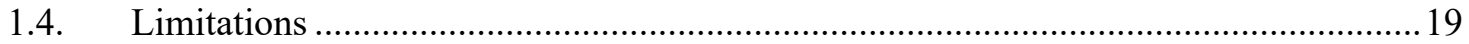

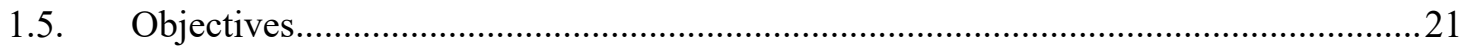

II. COMPUTATIONAL FLUID DYNAMICS MODELING …..............................22

2.1. Hybrid Zero-Equation \& Two-Equation Turbulence Models Coupling ........................24

2.2. Evaluation of Turbulence and Tile Modeling …………..............................................38

2.3. Composite Performance Index...............................................................................65

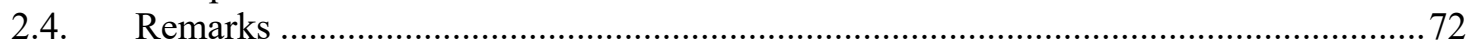

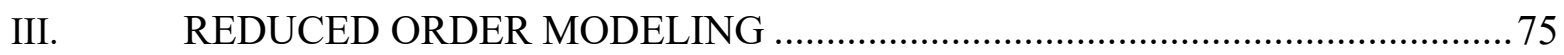

3.1. Proper Orthogonal Decomposition (POD) Method for Data Center Study ...................76

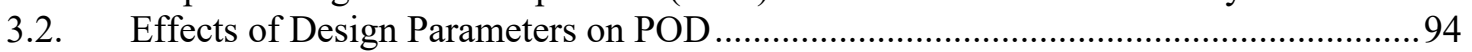

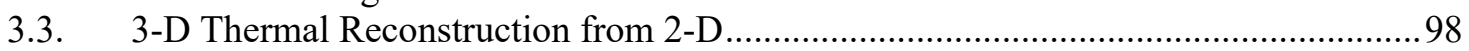

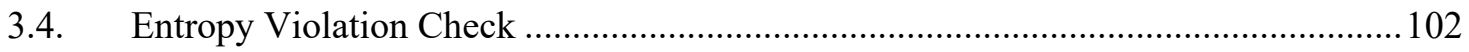

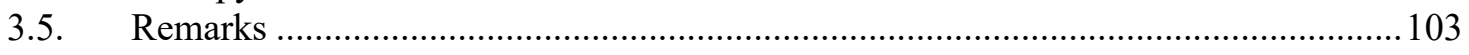

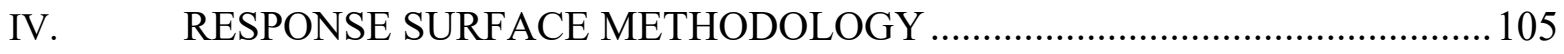

4.1. Response Surface Methodology (RSM) ………………....................................... 106

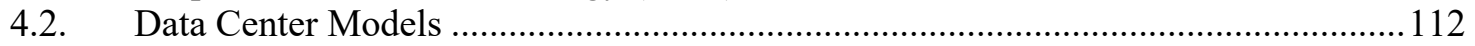

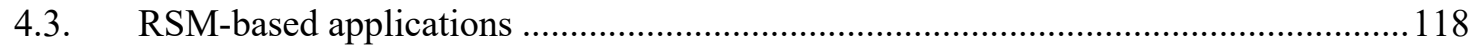

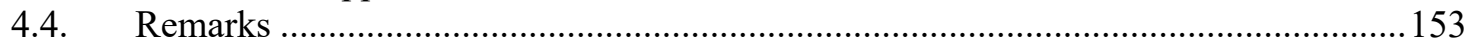

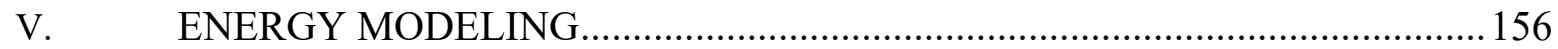

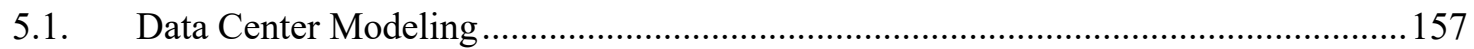

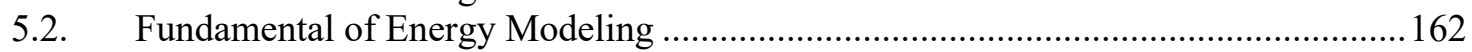

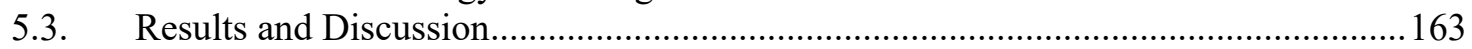

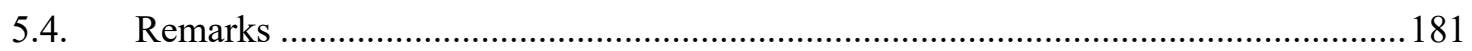

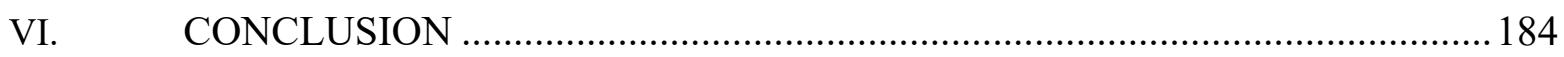

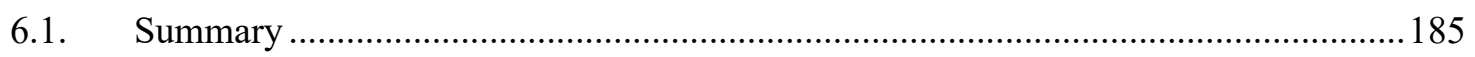

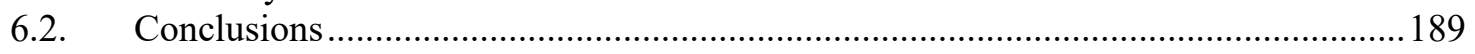

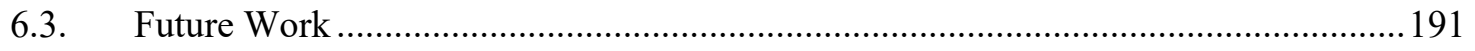

LIST OF REFERENCES ........................................................................... 192

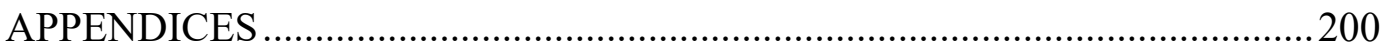

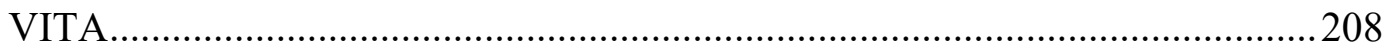




\section{LIST OF TABLES}

TABLE

PAGE

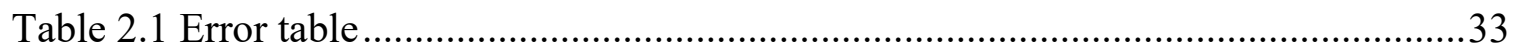

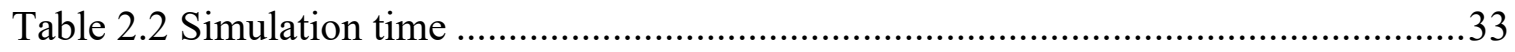

Table 2.3 The effect of inlet mass flow rate on viscous to non-viscous ratio ....................33

Table 2.4 The RSME of coupled method and standard k- $\varepsilon$ compared to Nielsen's data ...38

Table 2.4 Simulation time for different mesh sizes.................................................. 42

Table 2.5 Error assessment for different turbulence models at three flow ratios ..............66

Table 2.6 Error assessment for different tile models at three flow ratios........................67

Table 2.7 Non-dimensional index in terms of time and accuracy ................................. 70

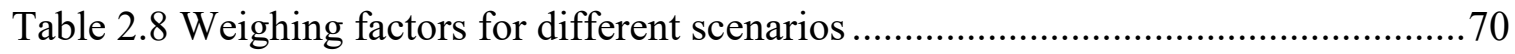

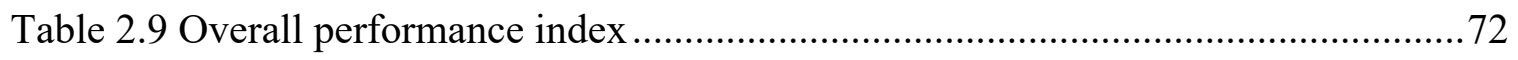

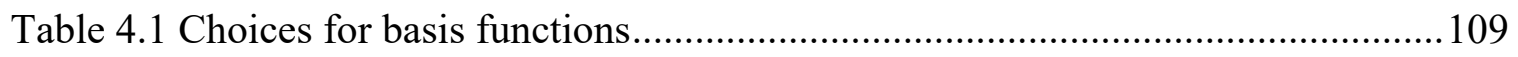

Table 4.2. Attributes of the genetic algorithm used during the optimization process ..... 117

Table 4.3. Performance measurement among different RBF models............................ 121

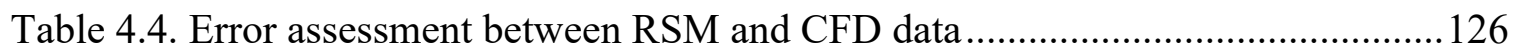

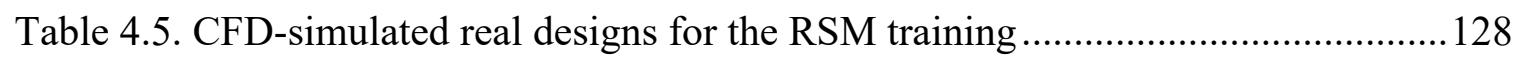

Table 4.6. Performance measurement among different RBF models for $\Delta \mathrm{T}$ output....... 128

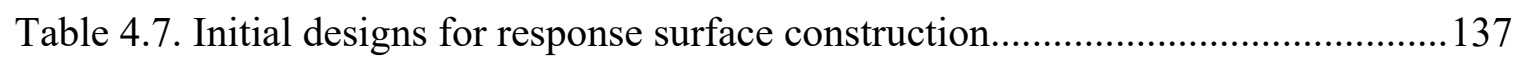

Table 4.8. RBF performance assessment for 4 different stages ................................. 139

Table 4.9. Targeted tile mass flow rate distribution at different shapes........................ 145

Table 4.10. Total time comparison between CFD and RSM methods.......................... 153

Table 5.1 Various surface exposure conditions of the data center model ..................... 159 
Table 5.2 Prescribed construction set for the current data center model........................159

Table 5.3 Multi-zone and single zone comparison in Miami and Chicago ..................... 166

Table 5.4 Comparison between two methods with CFD for adiabatic condition ...........166

Table A.1 Turbulence model governing equations and model constants......................200 


\section{LIST OF FIGURES}

FIGURE

PAGE

Fig. 1.1 A typical open-air data center with hot/cold aisle configuration ........................ 4

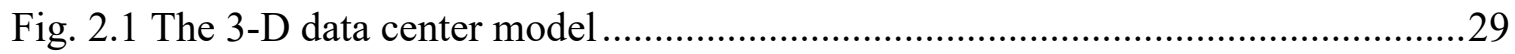

Fig. 2.2 Viscous and non-viscous regions formation .............................................. 30

Fig. 2.3 Whole domain comparison for different turbulence models............................32

Fig. 2.4 Coupled viscous and non-viscous domains temperature profile.........................34

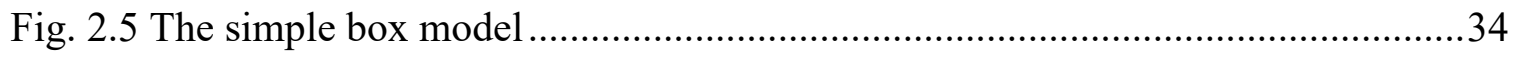

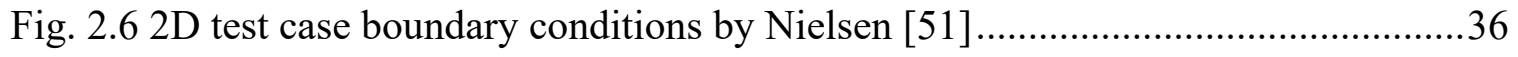

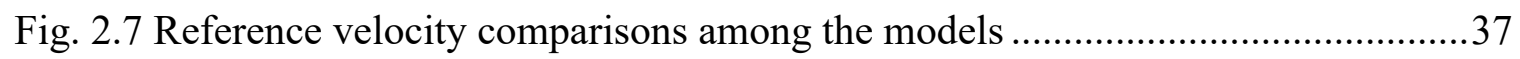

Fig. 2.8 Server rack model: a) geometrical dimensions b) boundary conditions.............40

Fig. 2.9 Mesh representations at various cell sizes ................................................. 41

Fig. 2.10 Velocities along the probing line for different mesh sizes.............................42

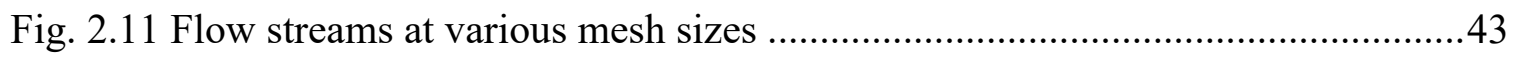

Fig. 2.12 One server rack model: detailed vs. simplified .........................................44

Fig. 2.13 Velocity after tile with and without tile model .........................................55

Fig. 2.14 Velocity streamlines after tile at three different flow ratios............................56

Fig. 2.15 Velocity contour for various tile models at three different flow rate ratios .......58

Fig. 2.16 Velocity after tile for different tile models at $20 \%$ flow rate ratio....................59

Fig. 2.17 Velocity after tile for different tile models at $60 \%$ flow rate ratio...................60

Fig. 2.18 Velocity after tile for different tile models at $100 \%$ flow rate ratio.................61

Fig. 2.19 After-tile velocities using BF tile model at different airflow rate ratios ............63

Fig. 2.20 After-tile velocities using MBF tile model at different airflow rate ratios ........64 
Fig. 2.21 After-tile velocities using measured velocity at different airflow rate ratios.....65

Fig. 2.22 Average time per iteration for different (a) turbulence models, (b) tile models 68

Fig. 3.1 Application of sweeping method a three-dimensional geometry........................82

Fig. 3.2 Hot and cold aisle practice in the current model .............................................. 83

Fig. 3.3 Cumulative energy percentage captured in each POD model............................ 88

Fig. 3.4 POD coefficients of different modes for four different test cases.......................89

Fig. 3.5 Mean temperature vs. modes..................................................................... 90

Fig. 3.6 Two- and three-parameter observations comparison at various design inputs ....91

Fig. 3.7 Average temperature difference between POD-CFD in the XZ plane................92

Fig. 3.8 Comparison among 2-observation POD, 3-observation POD, and CFD results .93

Fig. 3.9 Temperature variation across cold (left) and hot (right) aisles for case 1 ..........95

Fig. 3.10 CFD vs. POD, inlet temperature effect for case $1-3$ (mid plane)..................96

Fig. 3.11 CFD vs. POD, mass flow rate effect for case 8 - 10 (mid plane)....................97

Fig. 3.12 CFD vs. POD, server load effect for case 11 - 13 (mid plane)........................98

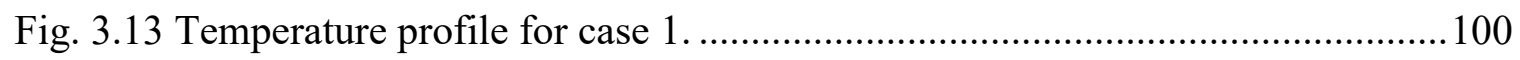

Fig. 3.14 Temperature profile comparison at location $\mathrm{Y}=1.5 \mathrm{~m}$ for different $\mathrm{d}^{*} \ldots \ldots \ldots 101$

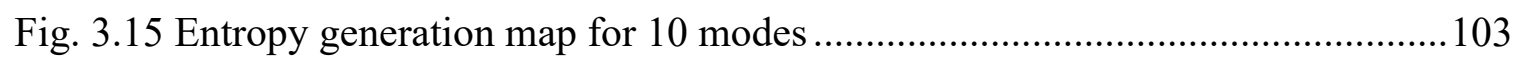

Fig. 4.1 The influence of shape parameter $\mathrm{cj}$ on multiquadrics RBF [18] ................... 110

Fig. 4.2. CFD-based RSM used in two applications for a data center model .................112

Fig. 4.3. A simplified data center overview floor plan with floor tiles shown...............114

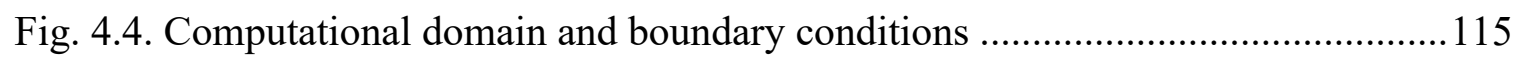

Fig. 4.5. Velocities along the probing line for different mesh sizes ........................... 117

Fig. 4.6. Random samples of 5\%, 10\%, and $20 \%$ of the original dataset ..................... 120 
Fig. 4.7. Distance chart comparison among 4 different RBF models

Fig. 4.8. Residual chart for Polyharmonic Spline

Fig. 4.9.RSM visualization for T vs. xz coordinates for only $20 \%$ training points 123

Fig. 4.10. Temperature comparison between response surface case and CFD. 125

Fig. 4.11. Distance chart comparison among 5 different RBF models 129

Fig. 4.12. RSM visualization for $\Delta \mathrm{T}$ versus different design parameters inputs 131

Fig. 4.13. The effect of each design parameter on the $\Delta \mathrm{T}$.

Fig. 4.14. Pareto designs 134

Fig. 4.15. Validation for the five selected Pareto designs 135

Fig. 4.16. Distance chart between real designs by CFD and virtual designs by RSM .... 138

Fig. 4.17. 3D response surface exploration for multiquadrics in the $1^{\text {st }}$ stage (modeFrontier)

Fig. 4.18. Optimum tile porosity combinations at different stages (modeFrontier)........ 141

Fig. 4.19. Design validation via stages.....

Fig. 4.20. Tile porosity for best-validated designs at each stage.

Fig. 4.21. Tile mass flow rate comparison 144

Fig. 4.22. Targeted shape for different tile mass flow rate distribution 145

Fig. 4.23. Tile porosity for best-validated designs at each stage 147

Fig. 4.24. Tile mass flow rate comparison 148

Fig. 4.25. Tile porosity for best-validated designs at each stage. 149

Fig. 4.26. Tile mass flow rate comparison 150

Fig. 4.27. Tile porosity for best-validated designs at each stage. 151

Fig. 4.28. Tile mass flow rate comparison 152

Fig. 5.1 Multi-Zone Data Center Model. 158 
Fig. 5.2 Single-Zone Data Center Model 164

Fig. 5.3 Hong's paper verification 165

Fig. 5.4 CFD and Energy Plus validation for the multi-zone model approach 167

Fig. 5.5 Monthly cooling electricity consumption for single zone and multi zone 167

Fig. 5.6 Monthly cooling electricity consumption between Miami and Chicago 168

Fig. 5.7 Zone mean air temperature at different zones..... 169

Fig. 5.8 Miami monthly temperature profile at different zones 171

Fig. 5.9 Chicago monthly temperature profile at different zones...... 171

Fig. 5.10 Winter design day for cooling electricity consumption. 172

Fig. 5.11 Summer design day for cooling electricity consumption..................................172

Fig. 5.12 Annual cooling load at different boundary conditions.....................................174

Fig. 5.13 Annual total load at different boundary conditions.........................................174

Fig. 5.14 Miami winter design day temperature profile ..............................................175

Fig. 5.15 Miami summer design day temperature profile ……………........................176

Fig. 5.16 The effect of supply temperature on energy consumption................................178

Fig. 5.17 The effect of volumetric flow rate on energy consumption .............................180

Fig. B.1 Optimal designs sort out at different stages .....................................................202

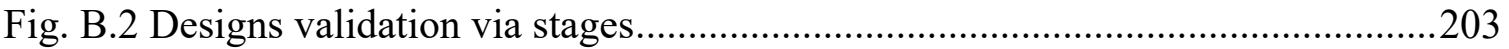

Fig. B.3 Optimal designs sort out at different stages ..................................................204

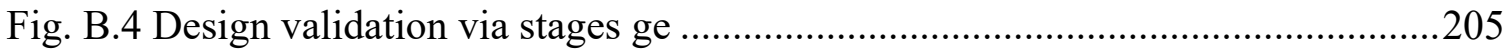

Fig. B.5 Optimal designs sort out at different stages ..................................................206

Fig. B.6 Design validation via stages …………….....................................................20 


\section{NOMENCLATURE}

\begin{tabular}{|c|c|}
\hline$\forall$ & Volume $\left[\mathrm{m}^{3}\right]$ \\
\hline$\nabla p$ & Pressure gradient \\
\hline$\overline{T^{\prime}}$ & Turbulent fluctuation temperature $\left[{ }^{\circ} \mathrm{C}\right]$ \\
\hline$\widehat{\dot{m}}$ & Predicted tile mass flow rate $[\mathrm{kg} / \mathrm{s}]$ \\
\hline$\overline{\dot{m}}$ & Average tile mass flow rate $[\mathrm{kg} / \mathrm{s}]$ \\
\hline $\bar{u}_{i}(\mathrm{~m} / \mathrm{s})$ & Average velocity vector \\
\hline$\|\cdot\|$ & The $\mathrm{L}^{2}$ norm \\
\hline$\overline{\mathrm{T}}$ & Average fluctuation Temperature $\left[{ }^{\circ} \mathrm{C}\right]$ \\
\hline$C_{T}$ & Sensible heat capacity multiplier \\
\hline$C_{p}$ & Zone air specific heat $[\mathrm{J} / \mathrm{g}-\mathrm{K}]$ \\
\hline$a_{m}^{(k)}$ & POD coefficient for snapshot number $k$, and the mode number $m$ \\
\hline$\dot{m}$ & Mass flow rate $[\mathrm{kg} / \mathrm{s}]$ \\
\hline$\hat{y}$ & Predicted value \\
\hline $\bar{y}$ & Mean value \\
\hline$\dot{M}$ & Objective function \\
\hline$\Delta y(m)$ & The total length in the y direction \\
\hline$A$ & Area of surface $\left[\mathrm{m}^{2}\right]$ \\
\hline $\boldsymbol{A}$ & Eigenvector \\
\hline$c$ & Shape parameter \\
\hline$C$ & Constant \\
\hline$C$ & Correlation matrix \\
\hline
\end{tabular}




\begin{tabular}{|c|c|}
\hline p & Air specific heat transfer capacity $\left[\mathrm{kJ} / \mathrm{kg}-{ }^{\circ} \mathrm{C}\right]$ \\
\hline$C_{z}$ & Air capacitance $\left[\mathrm{J} / \mathrm{m}^{3}-\mathrm{K}\right]$ \\
\hline$d$ & Distance $[\mathrm{m}]$ \\
\hline$D$ & Molecular diffusion term \\
\hline $\boldsymbol{E}$ & POD energy \\
\hline$e$ & Energy fraction \\
\hline$f$ & Function or air flow ratio \\
\hline$F$ & Production by system rotation or porosity \\
\hline$g$ & Gravitational acceleration $\left[\mathrm{m} / \mathrm{s}^{2}\right]$ \\
\hline G & Generation/Production term \\
\hline$h$ & Convective heat transfer coefficient $\left[\mathrm{W} / \mathrm{m}^{2} \mathrm{~K}\right]$ \\
\hline$k$ & Thermal conductivity $\left[\mathrm{W} / \mathrm{m} .{ }^{\circ} \mathrm{C}\right]$ or turbulence kinetic energy $\left[\mathrm{m}^{2} / \mathrm{s}^{2}\right]$ \\
\hline K & Pressure loss factor or total number of snapshots \\
\hline$L$ & Number of variables \\
\hline$M$ & Total number of POD mode \\
\hline$n$ & $\mathrm{~N}$ number of values or number of the primary selected planes \\
\hline$N$ & Number of enclosure surfaces \\
\hline$P$ & Number of input variables or points or stress production \\
\hline$p$ & Pressure $[\mathrm{Pa}]$ \\
\hline Q & Power $[\mathrm{W}]$ \\
\hline$q$ & Heat flux $\left[\mathrm{W} / \mathrm{m}^{2}\right]$ \\
\hline$r$ & Distance $[\mathrm{m}]$ \\
\hline$R^{2}$ & R-squared \\
\hline
\end{tabular}




\begin{tabular}{|c|c|}
\hline$S$ & Source term \\
\hline$S(\boldsymbol{P})$ & Series of points \\
\hline$S T D$ & Standard deviation \\
\hline$T$ & Tile porosity $[\%]$ \\
\hline$T$ & Temperature $\left[{ }^{\circ} \mathrm{C}\right.$ or $\left.\mathrm{K}\right]$ \\
\hline$V$ & Velocity $[\mathrm{m} / \mathrm{s}]$ \\
\hline$W$ & Weighing factor \\
\hline$x$ & $\mathrm{X}$ coordinate \\
\hline$y$ & Y coordinate or observed value \\
\hline$Y$ & Destruction term \\
\hline$y^{+}$ & Dimensionless wall distance \\
\hline \multirow[t]{2}{*}{$\boldsymbol{W}$} & Weight matrix \\
\hline & SUBSCRIPTS \\
\hline$\infty$ & Outside air \\
\hline$a$ & Accuracy-related \\
\hline$b$ & Baseline \\
\hline$B F$ & Body force \\
\hline desired & Desired temperature \\
\hline$i$ & $i$ th index \\
\hline in & Incoming tile velocity \\
\hline $\inf$ & Infiltration, surrounding air \\
\hline$j$ & $j$ th index \\
\hline$k$ & Turbulence kinetic energy \\
\hline
\end{tabular}




\begin{tabular}{|c|c|}
\hline$k$ & Snapshot number \\
\hline$L$ & $L^{\text {th }}$ index or molecular \\
\hline load & Zone load \\
\hline$m$ & Turbulence model or POD mode number or mean average \\
\hline$M B F$ & Modified body force \\
\hline neck & Vena contracta \\
\hline$p$ & Cutoff value \\
\hline pore & Pore tile velocity \\
\hline sup & Supply temperature \\
\hline sys & System \\
\hline$t$ & Turbulence or time-related \\
\hline tile & Tile-related \\
\hline$y$ & $Y$-direction \\
\hline$z$ & Zone \\
\hline $1-2$ & Between surface 1 and 2 \\
\hline $2-3$ & Between surface 2 and 3 \\
\hline $3-4$ & Between surface 3 and 4 \\
\hline & GREEK SYMBOLS \\
\hline$v$ & Kinematic viscosity $\left[\mathrm{m}^{2} / \mathrm{s}\right]$ \\
\hline$\alpha$ & Thermal diffusivity $\left[\mathrm{m}^{2} / \mathrm{s}\right]$ or coefficient $\alpha$ \\
\hline$\beta$ & Volumetric thermal expansion $[1 / \mathrm{K}]$ or coefficient $\beta$ \\
\hline$\delta$ & Partial derivative \\
\hline$\varepsilon$ & Rate of dissipation $\left[\mathrm{m}^{2} / \mathrm{s}^{3}\right]$ \\
\hline
\end{tabular}




$\begin{array}{ll}\eta & \text { Fraction of the time step } \\ \lambda & \text { Eigenvalue } \\ \mu & \text { Fluid dynamic viscosity }[\mathrm{kg} /(\mathrm{m} \cdot \mathrm{s})] \\ \xi & \text { Approximation function } \\ \rho & \text { Fluid density }\left[\mathrm{kg} / \mathrm{m}^{3}\right] \\ \sigma & \text { Prandtl number or Stephan Boltzmann constant } \\ \tau & \text { Reynold stress tensor } \\ \phi & \text { Pressure strain or POD mode } \\ \Xi & \text { Approximation function matrix } \\ \boldsymbol{\Phi} & \text { Basis function matrix }\end{array}$

\section{ABBREVIATIONS}

2D Two dimensional

3D Three dimensional

ASHRAE

American Society of Heating, Refrigerating and Air-Conditioning

Engineers

CFD Computational fluid dynamics

cfm Cubic feet per minute

CRAC Computer room air conditioner

$\mathrm{CRAH} \quad$ Computer room air handler

$\mathrm{ft} \quad$ Feet

in Inch

IT Information technology 


$\begin{array}{cl}\text { PIV } & \text { Particle image velocimetry } \\ \text { POD } & \text { Proper orthogonal decomposition } \\ \text { PUE } & \text { Power usage effectiveness } \\ \text { RMSE } & \text { Root mean square error } \\ \text { ROM } & \text { Reduced order model } \\ \text { RSM } & \text { Response surface methodology } \\ \text { SHI } & \text { Supply heat index } \\ \text { U } & \text { Rack unit }\end{array}$


CHAPTER I INTRODUCTION 


\subsection{Introduction}

A data center is a facility where information technology (IT) equipment such as computer servers and storage systems are hosted and to be used for telecommunications or many other purposes. The rapid development in IT in recent decades has offered people much better computing power and productivity. Almost all businesses, universities, media, governments, and many other sectors of the economy rely on the IT services provided from many data centers around the world. A data center's infrastructure includes many components, such as uninterruptible power supply (UPS) systems, and environment control systems (e.g., chiller plant, hot and cold pipeline, computer room air conditioner/handler, fire suppression system), and surveillance cameras. Since many sectors of the economy rely on the health of their computer servers, even a small downtime period could cause financial damages to their businesses. In addition, a network interruption is deemed a serious threat to one country's national security. Therefore, data centers are considered mission-critical facilities and constantly powered by redundant or backup power supply to ensure all online activities remain active.

A data center's energy consumption is typically huge compared to the energy use of other building types such as an office building. In fact, it was reported that a data center can consume up to 15 - 100 times more energy than a standard office building [5]. In addition, due to the constant power demand for a smooth uninterrupted operation, data center energy consumption is substantial in the total power consumption in the U.S. According to a latest report published in 2016, data centers in the U.S. consumed an estimated 70 billion $\mathrm{kWh}$ 
in 2014, representing about $1.8 \%$ of total U.S. electricity consumption [63]. A rapid development in information technology (IT) in recent decades has offered people with much better computing power and productivity, and yet demands higher power density in data centers. Indeed, in a report back in 2005 [29], ASHRAE estimated a heat load production footprint within 22 years (1992 to 2014) for a tenfold increase in power density.

There have been extensive efforts put forth to keep the energy consumption in data centers at a slower growth. These efforts include improved managements and to incorporate best practices in data centers. Other efforts such as virtualization and consolidation, which are expected to continue to generate energy savings and reduce power footprint. Finally, an energy efficiency trend study in 2016 shows that small to medium businesses start shifting their IT services and relying much on cloud computing, storage, and network services from trusted companies such as Amazon Web Services or Microsoft Azure [63]. These tech giants have the financial power and technology to integrate hyper-scale data center, and apply best practices in IT infrastructure management, which all helps reducing the energy consumption in U.S. data centers. Nonetheless, when data centers become larger in scale, the issue becomes much more critical in terms of thermal management.

A typical data center design is shown in Fig. I.1. Server racks are cabinets filled with rackmounted server at various heights measured in rack unit "U". One rack unit is equal to 1.75 inches. A standard server rack has an overall dimension of 24 inches wide by 39 inches deep by 78 inches tall. A fully filled rack can take up to $42 \mathrm{U}$ in height for a combination of various servers. 


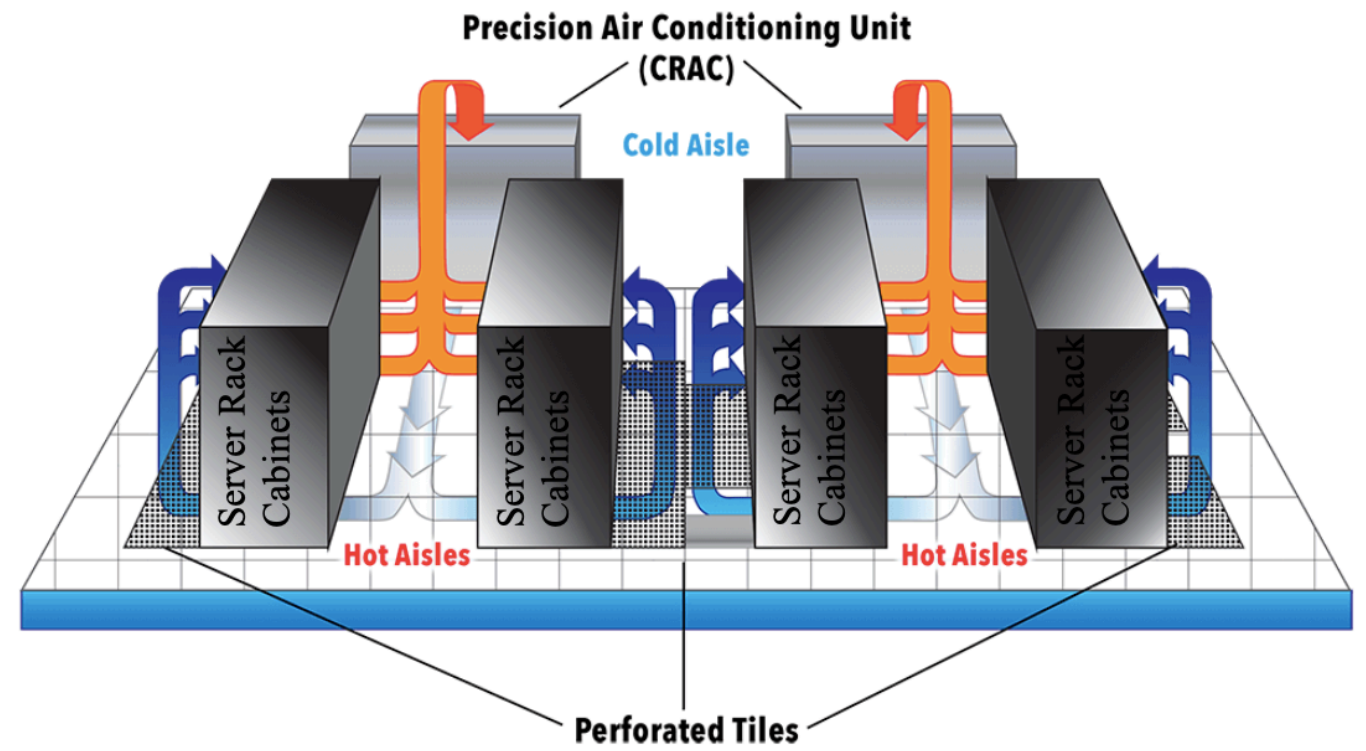

Fig. I.1 A typical open-air data center with hot/cold aisle configuration (Source: Severn Group)

For data center energy efficiency rating, power usage effectiveness (PUE) indicates the energy use in a data center. It is defined as the ratio of the total power entering a data center to the power used by the IT equipment (e.g., severs, storages, networks). Therefore, as the quotient decreases to unity, it shows that most of the energy is efficiently used for useful work in the IT equipment rather than in facility management such as cooling power and other overheads. In order to have PUE close to 1, data center thermal management needs to be improved in many aspects to bring down the energy consumption and thus drive efficiency.

As the thermal loads keep increasing to meet the high demand for IT services, it requires not only best practices in the design of data center infrastructures but also sound strategies for implementing different wise thermal solutions to bring data center's PUE close to unity. To that end, it requires modeling guidance for better thermal management of data center 
models. One of the computer-aided engineering tools that has been widely used in the research community for modeling such data center model is the Computational Fluid Dynamics (CFD) codes. These simulation programs are used in every step from design phase to operation phase, e.g., thermal monitoring and control in data center facilities.

\subsection{Background and Problem Definition}

During the past decades, data center energy use has become much more efficient thanks to the best-practice design adoption from the industry. In a real large-scale data center, thousands of server racks are arranged in rows in such a way that their front sides face each other to form cold aisles and the back sides are opposite to each other to form hot aisles. In cold aisles, perforated floor tiles are set up in front of the server racks to provide cold air from a typical two-foot deep raised floor plenum to cool hot servers. The structure of the underfloor plenum is supported either by round or square stanchions. Source of cold air is provided from computer room air-conditioners (CRAC) units for small office-size data centers or computer room air handlers (CRAH) units for large-scale data center facilities.

Optionally, hot air from the hot aisles is directed into the ceiling plenum and then back to the top of the CRAH unit. However, a majority of data centers are designed without the ceiling plenum allowing hot air to flow freely into the CRAH's top as seen in Fig. I.1. This open aisle configuration is prone to the hot/cold air mixing problem. When hot air recirculates into the cold aisle, it causes server hardware damage due to overheating (hot spots). This problem is even magnified in large-scale data centers having very long rows of server racks. The two ends of rack rows tend to receive less cold air and suffer much 
damage from hot spots. Moreover, the upper level of server racks is more likely to receive the hot air recirculation from the top of the racks causing cooling to be much less effective in this region. On the other hand, excessive cold-air provision from the underfloor plenum also causes the recirculation into hot aisles and results in a waste of energy utilization. The direction of hot and cold air recirculation is characterized by the tile-to-rack airflow ratio which is discussed detail in in Chapter 2.

Airflow through floor tiles plays an important role in cooling hot servers and also has a direct impact on the hot/cold air recirculation. The airflow rates coming out of the perforated floor tiles often experience non-uniformity with uniform floor tiles deployment. This problem is caused by the pressure drop when air moves through a clutter of things in in the underfloor plenum, e.g., stanchions, cables, pipes, and more. VanGilder and Schmidt [70] also addressed this issue and studied the root cause in the designs of various factors such as data center layouts, CRAH layouts, plenum obstructions, floor tile types, plenum depth, and many others. They concluded that perforated tile type and the presence of plenum obstructions have the greatest potential influence on airflow uniformity. In fact, these two major sources indicate the vital role of pressure loss in regulating the tile airflow distribution. Therefore, by controlling the pressure loss, one can obtain a nicely uniform distribution through perforated tiles. This is attainable either by adjusting the tile porosity or having the plenum obstruction arranged in a certain way to achieve the airflow uniformity. A novel methodology is proposed and discussed in Chapter 4 to optimize different sets of mixed-porosity floor tiles to achieve the desired airflow uniformity. In 
addition, the inverse design is also studied in this chapter allowing various airflow rates of choice to fit any supply air demand scenarios corresponding to various thermal loadings.

Data center designs nowadays rely much on the CFD tool to study various component features, e.g., floor tiles, server cabinets, underfloor plenum, CRAH units. Indeed, CFD is an excellent numerical tool for predicting the airflow and temperature profile inside data centers. It is relatively inexpensive when compared to costly experimental testing of data centers. Therefore, it is usually preferred in the early designing stages of new-built energyefficient data centers to avoid any future alterations due to bad designs. Besides, retrofitting old data centers by utilizing a CFD tool can also be a good option to lower the overall cost for business owners. Although CFD is a convenient and accurate analysis method for offline thermal management of data centers, the substantial amount of computing time and resource required are very large. This makes it very difficult or even unfeasible for generating thousands of design parameters in an optimization process. In the last few decades, the IT service increase has caused a huge surge in the load density of many data centers. This creates problems for cooling servers in existing data centers, and even a seasonal weather change could also impact the thermal management. The fluctuation in server loading during a normal service day is very common due to various reasons such as server traffic, server idling, server blanking-out, etc. and the cool air supply from a CRAH unit may not adequately meet the cooling requirement of computing servers. Indeed, for such online thermal assessments of data centers, a relatively slow and resource-intensive CFD tool becomes problematic. The need for a faster yet accurate methodology is indeed important for modeling data centers. It serves as the foundation for dynamic control and 
monitoring of modern data centers in today high demand of IT services, which has no room for signal delays or system shutdown due to the thermal issue.

\subsection{Literature Review}

Energy and thermal management in data centers is still an underlying problem in terms of both effectiveness and efficiency. Over the years, a great deal of efforts has been put into manipulating and improving the energy consumption. However, the enhancements are still limited, though a number of methods and strategies have been proposed by several researchers of various institutions. In this section, some related work on data center research regarding the computational methodologies used, the experimental testing efforts, and data center components modeling in predicting thermal and velocity profiles are of main focus.

\subsubsection{Computational Studies}

With the popularity of computational tools, data center performance improvements are more and more reliant on computer-based modeling and simulations, due to their cost effectiveness and quick turnaround time as compared to physical experiments or data collection. Computational fluid dynamics (CFD) are widely used as a powerful and convenient tool to study many components of data centers such as perforated tiles, server racks, underfloor plenums, etc. By solving Navier-Stokes equations of mass, momentum, and energy along with suitable numerical schemes, the airflow velocity, and thermal 
profiles of data center models can be resolved. These models give good predictions and useful insights while eliminating the expensive cost of performing experiments.

Sharma et al [62] defined a set of non-dimensional parameters and develop the heat indices based on CFD modeling of example data centers. They proposed supply and return heat indices (SHI and RHI) to address the hot and cold air mixing issues previously mentioned. The SHI is defined as the sensible heat gained ratio between the cold aisle and hot aisle. Lower values of SHI suggest a less mixing of warm and cold airstreams in the cold aisle. In contrast, the RHI is an index that measures the mixing level of returned warm air with supply cold air and is defined as the ratio of heat removal by the cooling system to the sensible heat gained at the rack exit. Higher values of RHI are desired because they show signs of less mixing of supply air with the warm returned air streams. Based on this study, some key conclusions for maximizing the performance in data centers are to avoid hot air recirculation into cold aisles, supply cold air mixed with return hot air, and short circuit of cold air back to the CRAC/CRAH's inlet.

In the early years, CFD was used to model high heat load data centers such as the work of Patel et al. [54]. In their study, a CFD model of a prototype data center constructed at Hewlett Packard in Richardson, TX was studied and validated. The study showed that CFD model was useful in detecting hot spots of the data center prototype model with 2 hot and 2 cold aisles, as well as predicting the supply inlet temperatures of the overhead air distribution system. However, the server racks were modeled as cuboids with volumetric 
heat source and flow rate specification, which still shows large error in the range of $11-$ $17 \%$ in average temperature.

The static provisioning of cooling system is only suitable for a well-defined and stable heat load specification. In reality, the thermal loading is constantly changing depending upon the demands. Moreover, in case of the cooling system failure, it creates even a much more thermal imbalance. In the attempt to resolve this thermal issue, Sharma et al. [61] used CFD to model a typical $2 \mathrm{hot} / 2$ cold aisle data center and provided two strategies in terms of power balancing, i.e., row-wise and regional thermal management. In the row-wise thermal management, they provided a power redistribution within rows of racks to tackle the hot spot issue normally occurred at the end of the aisles due to hot air recirculation. The regional thermal management was proposed to mitigate the thermal imbalance during the event of the cooling system failure. Overall, CFD was the vital tool to guide their research toward a dynamic thermal management strategy for data centers.

As for small-scale data center modeling, Iyengar et al. [36] constructed a small $50 \mathrm{ft} . \times 18 \mathrm{ft}$ $\times 10 \mathrm{ft}$ with only one standard 2-meter server rack. The power load was relatively high with $23 \mathrm{~kW}$, and the server was cooled by the underfloor air distribution unit. They used CFD tool to conduct the study and compare with the experimental data. The experimental part of the study was extensive spatial mapping of the temperature through measurement. The study showed a large discrepancy of $4^{\circ} \mathrm{C}$ in root mean square error (RMSE) and an average absolute temperature $3^{\circ} \mathrm{C}$. The reason was due to the lack of modeling strategies and the use of a very simple model to represent the server simulators. Following the work of 
Iyengar, Zhang et al. [86] proposed three different levels of rack modeling detail to study the same data center model. In addition, they studied a few of turbulence models, e.g., standard $k-\varepsilon$, L-VEL algebraic, Capped L-VEL in their CFD models. However, they failed to improve the error presented in Iyengar's study. They concluded that rack modeling detail had little influence on predicted temperatures. Uncertainties in room leakage and choice of turbulence had modest impact, and room size did not influence much on the temperature results. Finally, on the third attempt of correcting the thermal problem posed by Iyengar, Abdelmaksoud et al. [3] realized the importance of tile modeling and thermal buoyancy force. By incorporating different combinations of tile and buoyance force models, they claimed an improvement of $2^{\circ} \mathrm{C}$ in overall RMSE for the combination of buoyancy force and floor tile slot models. Their CFD analysis adopted the standard k- $\varepsilon$ turbulence model without any further justifications.

Most of the air flow in data center CFD modeling is considered turbulence flow. Past studies have presumably relied on the standard $k-\varepsilon$ turbulence model [3], [36], [14], [8], [9], [7], [81], [70]. This turbulence model is widely accepted in the industry due to its numerous advantages. Some of these include robust, good convergence, computationally cheap, and etc. Zhang et al. [86] suggested the insignificance of turbulence models on the thermal profile of the data center model they studied. However, their study only limited on only three turbulence models, i.e., standard $k-\varepsilon$, L-VEL algebraic, and Capped L-VEL, and did not extensively cover other more representative models. Therefore, in Chapter 2, a list of additional turbulence models is extensively studied and compared with one another. In 
addition, these turbulence models are proposed to be combined with various floor tile models in search for an optimum combination depending on user's modeling purposes.

\subsubsection{Data Center Component Models}

From previous literature review section, it can be seen that CFD tool is so powerful in studying multi-scale data center to predict the thermal and velocity profiles for various infrastructure designs and thermal management purposes. However, whole-scale data center CFD modeling is often relied on very simplistic models for data center component features such as cuboid model for the server rack. Though these models give a good approximation, important thermal aspects cannot be captured more accurately. Detailed studies of these features, on the other hand, are numerically prohibited due to high resolution of mesh size for capturing small features such as floor tile's tiny pores deployed in raised floor above the slab. Therefore, in recent years, researchers have focused on developing more simplified models for data centers features including server racks, floor tiles, floor plenum, computer room air handling (CRAH) unit to reduce the need for detailed modeling which tends to be very time-consuming.

Tile modeling is a very important aspect when it comes to modeling the airflow and the mixing level of hot and cold air streams in a data center. Tile modeling receives much attentions and has been improving over a decade. One of the early proposed tile models is the open tile model used in [1], [2], [3], [36]. This tile model assumes the airflow passes through the entire tile area (fully open) or a partial tile area (single open). Though it is the simplest way to model perforate floor tiles, it usually under predicts in the case of fully 
open tile model or over predicts in the case of single opening tile model. Because the pressure loss through perforated tiles is one of the main concerns in tile modeling, porous jump $(P J)$ model [8], [7], [9] was proposed to introduce the pressure loss while keeping the tile model as much simple as a 2-D porous jump. With pressure loss treatment alone cannot correct the exact velocity magnitude in the cold aisle, Abdelmaksoud et al. [2] proposed and validated different tile models and also provided improved tile models such as body force (BF) model, and multiple openings tile model. The idea is to add a momentum enhancement on top of the $P J$ model to resolve the momentum deficit in previous tile models. These models showed good agreements with experimental data when applied in lab-scale data centers [1], [3]. Arghode et al. [8], [7], [9] followed up with Abdelmaksoud's study by introducing a modified body force (MBF) model and provided a detailed analysis for obtaining the momentum source region dimensions and other associated input variables for the both $B F$ and $M B F$ models. The $B F$ and $M B F$ arguably resolve the flow physics of air above perforated tiles. Arghode et al [9] compared different tile models and showed good fit for airflow patterns in the rack front's region, especially with the $M B F$ model. However, they did not explicitly show the velocity magnitudes or quantify the modeling errors as compared to the experimental results shown in [41].

As for rack modeling, server racks were represented with volumetric heat blocks with prescribed air flow rates by some researchers [36], [54], [61]. Zhang et al [86] studied three different rack models based on the level of rack details and concluded that rack modeling detail has little influence on predicted temperatures. They also introduced the concept of black-box model (BBM) for rack modeling. Zhai et al. [81] later examined more carefully 
the BBM by paying attention to the translation of temperatures and velocity values from front plate to rear plate of a sever rack. They also compared this BBM to an interim model called open-box model and obtained an improved result. Both models are designed to be simple and require minimal user inputs, therefore, are suitable for CFD modeling.

When deploying a set of uniform floor tiles with the same porosity, non-uniformities in tile airflow are often encountered. The level of airflow uniformity depends on many factors, e.g., the raised floor depth, the size of a data center, or the distance from the CRAC/CRAH to the layout position of perforated tiles. Since tile airflow has a direct impact on server racks, the need for controlling the floor tile airflow distribution is of importance in data centers. For better understanding of the cause of non-uniformity in floor tile airflow, VanGilder and Schmidt [70] studied airflow uniformity through perforated tiles in 240 CFD data center models. They concluded that perforated tile type and the presence of plenum obstructions had the greatest potential influence on airflow uniformity. Type of perforated tiles used such as ones with different porosities and plenum obstruction such as cables, stanchions, pipes, etc. account for the pressure loss across the raised-floor plenum. In fact, these two major sources indicate the vital role of pressure loss in regulating the tile airflow distribution. Therefore, by controlling the pressure loss, one can obtain a nicely uniform distribution through perforated tiles. This is attainable either by adjusting the tile porosity or having the plenum obstruction arranged in a certain way to achieve the airflow uniformity. For better modeling of the plenum obstructions, VanGilder et al. [70] studied a compact stanchion model by introducing the loss coefficient which can reduce the computational overhead and is easy to specify in CFD tools. Fulpagaree et al. [26] studied 
the effect of plenum chamber obstructions and concluded that up to $80 \%$ in air flow rate decrease and up to $2.5^{\circ} \mathrm{C}$ increase in temperature are caused by such obstructions. The situation involving plenum obstruction is usually very complicated and not easy to control due to the layout of the plenum construction, e.g., piping systems, cables, stanchions, etc. Perhaps, a more manageable way to deal with the airflow non-uniformity is through tile selection and arrangement.

\subsubsection{Faster CFD Methods}

Recently, some faster CFD methodologies have been extensively studied for reducing simulation time and utilizing less computing resource. In a simplified approach, inviscid and potential modeling [32], [45], [16], [72], [73] in which no turbulence model is specified provides an exact result for irrational inviscid flow. However, these models are just an approximation and normally fail to represent a more complex flow, especially in the regions where strong vorticity effects are of dominance. Some other researchers endorse coarse grid used in CFD method for indoor air environment studies such as Wang et al. [75], [76]. However, these studies need to be further justified due to the concerns of its accuracy. Other attempts to reduce the computing time of the CFD simulation include the innovative fast CFD method developed by Zou et al. [37], [88]. In recent year, reduced order modeling (ROM) of data centers has constantly attracted many researchers because of its capability to reduce a more complex heat transfer (HT)/CFD model to a computationally inexpensive surrogate model for time saving. Among these ROMs, proper orthogonal decomposition (POD) method using snapshots has gained popularity in many new studies related to data centers with good accuracy while saving much computing time. 
Although POD method has extensively used in other fields under various names, it is relatively new when it comes to data center research. By investigating different design parameters, Samadiani and Joshi [58], [59], [60] were able to use POD to reconstruct the airflow and temperature fields inside a data center model. Li et al [46] couple POD model with genetic algorithm for an optimization study of a ventilation system inside an office. Many design objectives are sought to be minimized including fan power, $\mathrm{CO}_{2}$ concentration, cooling energy requirement. However, only one single objective function is prescribed by aggregating and weighting indices, which is not recommended in multiobjective due to its unreliability and inconsistency. Though POD method reduces the time effort for traditional HT/CFD simulation with good accuracy, there still exists some weaknesses. For instance, the method normally requires large number of sensors input to construct for its basis. In addition, it is very sensitive to new changes of a data center's design input and/or geometry, which demands new observations to be in place. The accuracy totally depends on the quality of CFD observations.

Response surface methodology (RSM) is in essence a multi-dimensional data fitting method used to approximate empirical data. RSM relates several independent input variables to an output known as a response. The process is relatively fast and computationally efficient, therefore, RSM is usually employed during an optimization process to generate multiple virtual designs without the need for all of the high-cost computational analyses. RSM was first introduced by Box and Wilson [10] back in 1951. RSM has recently gained popularity in many applications including sheet metal forming [35], chemical composition of new superalloys [22], and improved RSM for faster 
engineering application approximations [74], [50]. RSM can provide useful accurate information while using less computational resource. In recent studies, Colaço \& Dulikravich have developed the RSM-based hybrid optimizer capable of interpolate both linear and non-linear functions in multi-dimensional spaces with up to 500 dimensions [19], [17], [20]. They also performed an in-depth comparison of various deterministic and stochastic optimization techniques, as well as RSM techniques in complex high dimensionality problems [18]. These studies are particularly useful for many inverse design engineering applications with highly accurate and small computing time. Among the methods for constructing an RSM model, radial basis function (RBF) is more preferable as a basis function to construct response surfaces due to its high accuracy, yet less mathematical computing effort. The use of RBFs followed by collocation is a technique first proposed by Kansa [39] after the work of Hardy [30] on multivariate approximation. RSM does not require a large data set to train the response surface. In fact, it can work with a sparse data set to interpolate more extra points (virtual data points). One advantage while using RSM is the ability to extrapolate information outside the domain of interest within less than $5 \%$ of the original dataset [56]. In regard to data center or building research, RSM has not extensively been explored. Phan and Lin [55] attempted to use RSM to optimize various design input parameters, as well reconstructed the airflow and temperature fields of a data center model [56]. The latter research is potentially useful for real-time thermal monitoring of data centers. 


\subsubsection{Energy Modeling}

In the data center modeling and simulation, computational fluid dynamics (CFD) programs can be used as analytical tools to obtain detailed thermal/fluid transport information about the data center, such as air velocity and temperature distributions, thermal comfort, as well as indoor air quality. Building energy simulation (BES) programs, on the other hand, can be used to obtain information on the overall performance of the whole building, as wells as providing information on the heating, ventilating and air conditioning (HVAC) system sizing, e.g., cooling/heating loads, energy consumption on an hourly or sub-hourly basis for a certain period of time (days, months, weeks, or years). Although CFD programs are able to provide three-dimensional thermal fields, their limitations in practical applications are still present in terms of meshing, running time, and computational resources, particularly for transient problems. The BES programs, though giving less detailed results inside the building, can provide quick insights into the overall building energy behavior with change of time [15], [85], [82], [83], [84]. A literature survey shows that although building energy simulation can be used to estimate annual energy consumption of any building, its application in data centers has been very limited in open literature. Hong et al. [33] compared the HVAC simulations between EnergyPlus and DOE-2.2 for a data center. They found that annual cooling electricity consumption calculated from both programs were reasonably matched within a range of $-0.4 \%$ to $8.6 \%$. In another study, Pan et al. [53] utilized EnergyPlus to compare the energy saving options of two office buildings housing IT equipment in Shanghai, China with two building models. They have found that the proposed design options can save energy up to $27 \%$ from China Code building and $21 \%$ from AHSRAE budget building. 


\subsection{Limitations}

Although CFD is a convenient and accurate analysis method for offline thermal management of data centers, the substantial amount of computing time and resource required are very large. This makes it very difficult or even unfeasible for generating thousands of design parameters in an optimization process. In the last few decades, IT service increase causes a huge surge in load density of many data centers. This creates problems for cooling servers in existing data centers, and even a seasonal weather change could also impact the thermal management. Indeed, the fluctuation in server loading during a normal service day is very common due to various reasons such as server traffic, server idling, server blanking-out, etc. and the cool air supply from a CRAH unit may not adequately meet the cooling requirement of computing servers. In order to tackle these issues, the need for a more responsive real-time cooling system is of paramount importance. Indeed, for such online thermal assessments of data centers, relatively slow and resource-intensive CFD tool becomes problematic.

However, none of these existing studies stated specifically why a turbulence model is particularly preferred for data center research or provided proven comparisons with other turbulence models. Although there are some available turbulence model studies for indoor air environment research such as the one in [87], different turbulence models have not been exclusively studied in data center research. Data centers are much different from standard offices. They have considerably high thermal density and active regions like cold aisles and racks with strong flow streams. Therefore, adopting only standard $k-\varepsilon$ turbulence model needs a clear justification to validate the outcome of such model. 
All of the tile models studied in the literature are to eliminate the need for detailed modeling of the perforated structures, thus, allowing larger data center layouts to be studied and significantly reducing the computational cost. The $B F$ and $M B F$ models arguably resolve the flow physics of air above perforated tiles. Arghode et al [9] compared different tile models and showed good fit for airflow patterns in the rack front's region, especially with the $M B F$ model. However, they did not explicitly show the velocity magnitudes or quantify the modeling errors as compared to the experimental results shown in [41]. In addition, a performance index for tile models needs to be developed to evaluate the fidelity of each tile model. This performance index should be analyzed concurrently with the best turbulence model studied. In other words, a combination of best turbulence model and tile model should be assessed via some form of performance index for the purpose of accurate and efficient CFD modeling for data centers.

All of the existing studies regarding the building energy modeling have adopted the well mixed single-zone approach, without considering the existence of hot and cold aisles in the data center. Considering the importance of modern data centers, it is obvious that more studies on data center's performance are required using an improved data center modeling approach. 


\subsection{Objectives}

Within the scope of developing new methods for accelerating the computing speed and maintaining a reasonable accuracy for data center modeling, the objectives of this dissertation work can be outlined as follows:

- The first objective is to couple the zero-equation and the two-equation turbulence models by partitioning the computational domain into viscous and non-viscous zones. (Chapter 2, section 2.1)

- The second objective is to assess various turbulence models along with data center feature models in search of the best combination to be applied in data center modeling. (Chapter 2, section 2.2). As a result, a composite performance index will be developed to guide the appropriate method selection based on different modeling purposes (Chapter 2, section 2.3).

- The third objective is to study reduced order modeling exclusively for data centers. Proper Orthogonal Decomposition (POD) is used to propose a 2D-based modeling method for a 3D data center model construction. The effects of multiple design parameters on the POD method, including inlet air mass flow rate, rack heat load, and inlet air temperature are also studied to assess the fidelity of the POD model (Chapter 3).

- The fourth objective is to study response surface methodology (RSM) for the first time in thermal management of data centers. Due to its rapid running time, RSM can be used to accommodate the optimization of multiple design parameters where thousands of virtual design scenarios need to be generated. In addition, RSM can 
also be applied to reconstruct the airflow and temperature fields quickly, which is potentially useful for real-time thermal monitoring of data centers. Multiple applications of RSM for data centers are carefully studied including data center thermal profile reconstruction, minimization of both the temperature difference across server racks and the maximum temperature within a data center model, and mixed floor tile selection (Chapter 4).

- The final objective is to propose a new multi-zone modeling strategy to study the energy consumption of a typical hot/cold aisle data center model. A parametric study is conducted to study the effects of five different surface boundary conditions on the cooling electricity consumption and the zone mean air temperature in the data center in two typical U.S. climates. In addition, the effects of both supply temperature and volumetric flow rate of the supply side are also investigated. 
CHAPTER II COMPUTATIONAL FLUID DYNAMICS MODELING 


\subsection{Hybrid Zero-Equation \& Two-Equation Turbulence Models Coupling}

In this section, we will explore an alternative strategy within the similar blending ideas from the coupled viscous and non-viscous domains. The "non-viscous domain" referred to in this section is the region where there is insignificant and less turbulent flow characteristics. Such region can be modeled using simpler models such as potential flow, inviscid flow, etc. In this study, we use the zero-equation model in these low to non-viscous regions in order to avoid the drawbacks of inviscid modeling with a similar technique that divides the computational domain in two regions, i.e., viscous and non-viscous regions. The zero-equation model has certain benefits in indoor airflow modeling because it uses much less computer memory and the computing speed is at least 10 times faster, compared with the $k-\varepsilon$ model when grid size substantially reduced [13]. However, we cannot totally rely on the zero equation alone in a whole domain simulation due to some drawbacks. For instance, estimation of the mixing length is difficult and depends on each problem, and it is completely incapable of describing flows with separation or circulation, which is an important feature in data centers. In the viscous region, the standard $k-\varepsilon$ turbulence model will be used, which will be coupled with the zero-equation model. Detailed step-by-step procedures on how to divide such regions are discussed. In addition, a parametric study is carried out to study the effect of inlet mass flow rate on the volume ratio of the two regions using a simple box model. 


\subsubsection{Methodologies}

\section{a. Overall Coupled Viscous and Non-Viscous Solution Method}

The overall solution method is divided into four major steps. In the first step, the standard $k$ - $\varepsilon$ turbulence model with a coarse mesh is used for solving the whole domain of the data center model. The result from this preliminary run sets up a basis for the viscous and nonviscous region division technique. The vorticity magnitude is thereby calculated and set as an indicator for viscous domain detection. In the second step, the viscous, interzone, and non-viscous regions are created based upon the vorticity magnitude level. The detailed partitioning technique is discussed later in the section. In the third step, after the viscous and non-viscous domains have been identified, the first run of viscous region is performed using the standard $k-\varepsilon$ turbulence model. The boundary conditions for the viscous run are then obtained from the preliminary run in the first step. In the fourth step, the non-viscous run is started with the boundary conditions supplied from the viscous run in the second step. In the subsequent runs, the viscous and non-viscous domains are alternately calculated until a convergence point is reached. The boundary conditions are passed back and forth from viscous to non-viscous and vice versa via the interzone. Convergence is reached when there is no change in the overall temperature profile or is a minimal value compared to the prescribed tolerance. The whole calculation process is then stopped, and the results are ready to be analyzed. 


\section{b. Whole Domain Numerical Solution}

The fundamental Reynolds average Navier-Stokes (RANS) equations (eq. (II.1) - (II.3)) for viscous flows are solved. The turbulence models that are present in this section include standard $k-\varepsilon$ [44], realizable $k-\varepsilon$ [64], Spalart-Allmaras [68], and zero-equation [13] models. Initially, all of the turbulence models are simulated independently and compared among one another for accuracy and speed assessment before being chosen for the coupled viscous and non-viscous solution method.

$$
\begin{aligned}
& \frac{\partial}{\partial x_{i}}\left(\bar{u}_{i}\right)=0 \\
& \rho \frac{\partial}{\partial t}\left(\bar{u}_{i}\right)+\rho \frac{\partial}{\partial x_{j}}\left(\bar{u}_{i} \bar{u}_{j}\right)=-\frac{\partial \bar{P}}{\partial x_{i}}+\mu \frac{\partial^{2} \bar{u}_{i}}{\partial x_{j} x_{j}}+\frac{\partial \tau_{i j}}{\partial x_{j}}-\beta \rho\left(\bar{T}-T_{o}\right) g_{i} \\
& \text { where, } \tau_{i j}=-\rho_{o} \overline{u_{\imath}^{\prime} u_{\jmath}^{\prime}}=\left|\begin{array}{lll}
\overline{u^{\prime 2}} & \overline{u^{\prime} v^{\prime}} & \overline{u^{\prime} w^{\prime}} \\
\overline{u^{\prime} v^{\prime}} & \frac{v^{\prime 2}}{u^{\prime} w^{\prime}} & \frac{\overline{v^{\prime} w^{\prime}}}{v^{\prime} w^{\prime}}
\end{array}\right| \text { and } \beta=-\frac{1}{\rho}\left(\frac{\partial \rho}{\partial T}\right)_{p} \\
& \frac{\partial \bar{T}}{\partial t}+\frac{\partial \overline{u_{l}} \bar{T}}{\partial x_{i}}=\alpha \frac{\partial^{2} \bar{T}}{\partial x_{i} x_{i}}+\frac{\partial \overline{u_{l}^{\prime} T^{\prime}}}{\partial x_{i}} \\
& \text { where, } \alpha=\frac{k}{\rho C_{p}}, \overline{u_{l}^{\prime} T^{\prime}}=-\alpha_{t} \frac{\partial \bar{T}}{\partial x_{i}}, \alpha_{t}=\frac{v_{t}}{\sigma_{t}}\left(0.7<\sigma_{t}<0.9\right)
\end{aligned}
$$

The standard $k-\varepsilon$ turbulence model is used in the viscous zone and as a baseline model for comparisons with other models. In this model, two additional equations, i.e., $k$-equation (eq. (II.4)) and $\varepsilon$ equation (eq. (II.5)) are also solved to obtain the turbulence viscosity as seen in eq. (II.6).

$$
\begin{gathered}
\frac{\partial}{\partial t}(\rho k)+\frac{\partial}{\partial x_{i}}\left(\rho k \bar{u}_{i}\right)=\frac{\partial}{\partial x_{j}}\left[\left(\mu+\frac{\mu_{t}}{\sigma_{k}}\right) \frac{\partial k}{\partial x_{j}}\right]+G_{k}+G_{b}-\rho \varepsilon \\
\frac{\partial}{\partial t}(\rho \varepsilon)+\frac{\partial}{\partial x_{i}}\left(\rho \varepsilon \bar{u}_{i}\right)=\frac{\partial}{\partial x_{j}}\left[\left(\mu+\frac{\mu_{t}}{\sigma_{\varepsilon}}\right) \frac{\partial \varepsilon}{\partial x_{j}}\right]+C_{1 \varepsilon} \frac{\varepsilon}{k}\left(G_{k}+C_{3 \varepsilon} G_{b}\right)-C_{2 \varepsilon} \rho \frac{\varepsilon^{2}}{k} \\
\mu_{t}=\rho C_{\mu} \frac{k^{2}}{\varepsilon}
\end{gathered}
$$


where, $G_{k}=-\rho \overline{u_{\imath}^{\prime} u_{\jmath}^{\prime}} \frac{\partial \bar{u}_{j}}{\partial x_{i}}$ representing the production of turbulence kinetic energy. $G_{b}=$ $\beta g_{i} \frac{\mu_{t}}{P r_{t}} \frac{\partial \bar{T}}{\partial x_{i}}$ representing buoyancy turbulence generation term. $C_{1 \varepsilon}, C_{2 \varepsilon}$, and $C_{3 \varepsilon}$ are constants. $\sigma_{k}$, and $\sigma_{\varepsilon}$ are the turbulence Prandtl numbers.

The following empirical turbulence model constants were used $\mathrm{C}_{\mu}=0.09, \mathrm{C}_{1 \varepsilon}=1.44, \mathrm{C}_{2 \varepsilon}=$ $1.92, \mathrm{C}_{3 \varepsilon}=0.09$. Standard wall functions were used in this turbulence model. The phase coupled SIMPLE algorithm and least squares cell based were used for the velocity-pressure coupling and the gradients in spatial discretization, respectively. For momentum, turbulent kinetic energy, and turbulent dissipation rate equations, the first order upwind schemes were used. Numerical computations were carried out using a popular commercial CFD package [6].

The zero-equation is particularly used in the non-viscous region for a faster solution time. In this model, there is no PDE that describes the transport of the turbulent stresses and fluxes. A simple algebraic relation is used to close the problem. Based on the mixing length theory, which is the length over which there is high interaction of vortices in a turbulent flow field, dimensional analysis was used to show that:

$$
v_{t}=\frac{\mu_{t}}{\rho} \sim l u=l_{m}^{2}\left(\frac{d U}{d y}\right)
$$

where $l_{m}$ is the length scale and usually determined experimentally for basic geometries. For boundary layers, we assume the followings [24],

$$
l_{m}=\kappa y \text { for } y<\delta \text { and } l_{m}=\kappa_{o} \delta \text { for } y \geq \delta
$$

where $\delta$ is the boundary layer thickness, $y$ is the wall distance, $\kappa$ is the von Karman constant $(0.41), \kappa_{o}=0.09$ 


$$
k_{t}=\mu_{t} \frac{c_{p}}{P r_{t}}
$$

Eqs. (II.7) - (II.9), are then used in the laminar-turbulent analogy and then back into the original RANS equations.

\subsubsection{Coupled Viscous and Non-Viscous Calculation}

\section{a. Data Center Model}

The data center model as seen in Fig. II.1 was adopted from the one that has been used by previous studies [61], [4], [48], [49]. The dimensions of the data center are $45.9^{\prime} \times 32.8^{\prime} \times$ $11.5^{\prime}(14 \mathrm{~m} \times 10 \mathrm{~m} \times 3.5 \mathrm{~m})$. The data center has a total of 1120 server blades which are

distributed into four rows of rack unit. Each row consists of seven $40 \mathrm{U}$ racks (1U equals $45 \mathrm{~mm}$ ). Power dissipation per rack is specified as $3.66 \mathrm{~kW}$. There are four CRAC (computer room air conditioner) units placed in the middle of four different walls with a cooling capacity of 100 tons per CRAC. Each CRAC provides about 9,132 CFM to the pressurized 2-foot raised floor plenum. Each server receives 32.5 CFM of cool air from the fully open restrictive perforated floor tiles. The data center was configured using the current best practice of hot and cold aisles. The server racks are placed in such a way that their back sides are facing each other to form hot aisles, while the front sides, which receive cooling air from the low-flow perforated floor tiles, form cold aisles. 


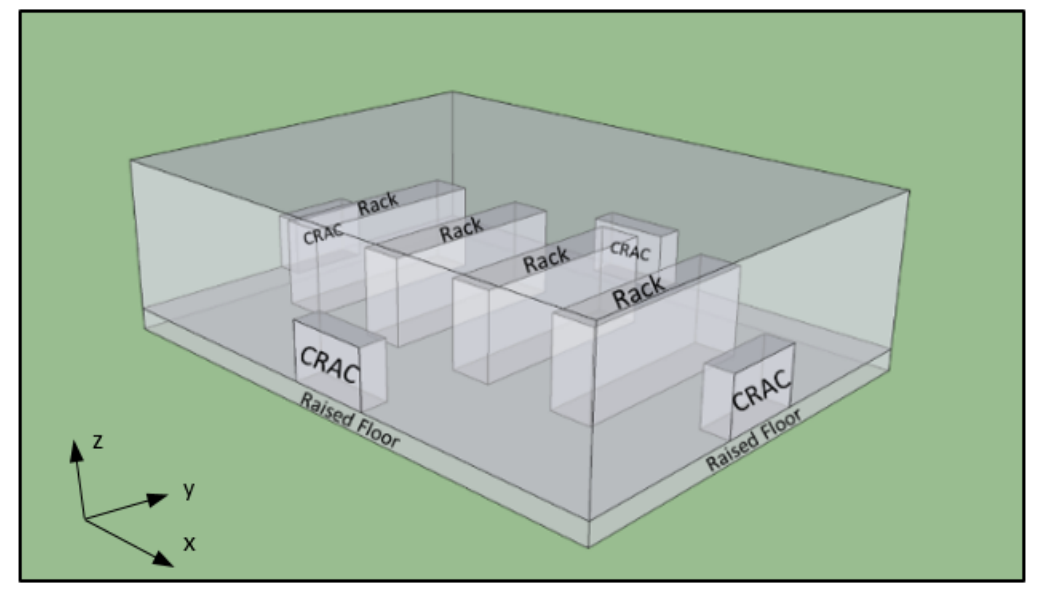

Fig. II.1 The 3-D data center model

4 CRAC (Computer room air-conditioner) units, 4 rows of server racks

\section{b. Partitioning Process}

The partitioning procedure starts up with a preliminary run that used a medium mesh and the standard $k-\varepsilon$ turbulence model. Upon completion, the vorticity magnitude $(|\overrightarrow{\boldsymbol{\omega}}|)$ was calculated throughout the entire computational domain. It was then split into two bounded range as follows,

$$
\left\{\begin{array}{cc}
\text { Viscous domain, } & \text { if }|\vec{\omega}|>1.5 \\
\text { Non-viscous domain, } & \text { if }|\vec{\omega}| \leq 1.5
\end{array}\right.
$$

In order to detect viscous regions, an algorithm was developed to sweep through the 3-D domain of the data center model to search for matching conditions prescribed above. The sweeping process consists of two steps. Both $x$ and $y$ directions were involved in the search. As we moved along the $x$-direction of the data center, the algorithm marked the areas where the vorticity magnitude is greater than 1.5 and stored in the memory. Another constraint is that the algorithm only picked up the nearby grid cells and neglected the scattered ones. This ensures a continuously connected viscous region in the end. Likewise, in the $y$ - 
direction, the sweeping algorithm was processed in the same manner. The result is a connected viscous region throughout the entire domain. After the viscous region has been generated, the next steps were to create the interzone and non-viscous regions. The viscous region was moved inward as a small distance equal to $0.1 \mathrm{~m}$ on the vertical surfaces and $0.2 \mathrm{~m}$ on the top horizontal surface near the ceiling as seen in Fig. II.2b.

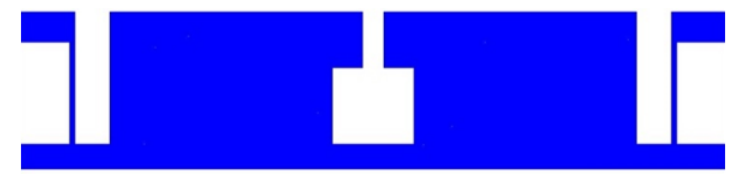

a) Viscous zone + interzone

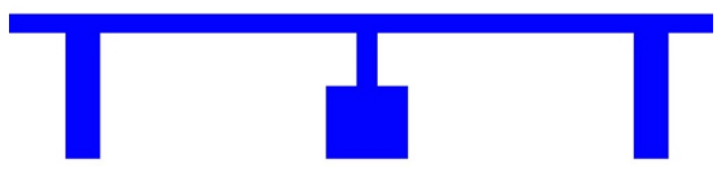

c) Non-viscous zone

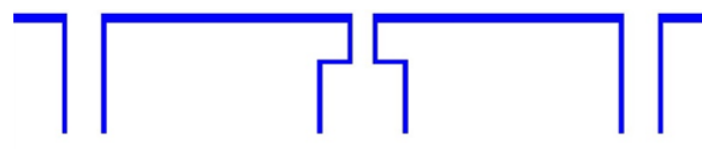

b) Interzone

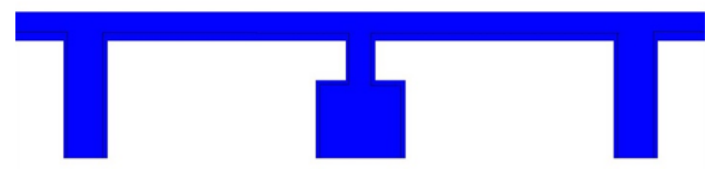

d) Non-viscous zone + interzone

Fig. II.2 Viscous and non-viscous regions formation viscous domain $=$ viscous zone + interzone; non-viscous domain $=$ non-viscous zone + interzone

The viscous zone combined with the interzone forms the viscous domain, and the nonviscous zone combined with the interzone forms the non-viscous domain. The interzone serves as an intermediate zone, which is used to pass on the boundary conditions between both viscous and non-viscous domains.

\section{c. Grid Sizing}

Initially, the mesh was generated with a medium mesh size for the entire computational domain with a total of 422,320 grid cells. The standard $k-\varepsilon$ turbulence model was used 
for preliminary simulation to obtain the vorticity magnitude level. The vorticity magnitude level is serves as a basis for zone partitioning. The reasons to use this model is that it is proven with reasonable accuracy. It is very robust, economical, thus, popular for flows that have complex geometries and heat transfer. It is suitable for applications that have relatively small pressure gradients, and data center applications generally conform to this assumption.

In the whole domain calculation, the mesh independence study was carried out. It was observed that 1,553,231 grid cells led to a very small further change in maximum temperature. Therefore, the mesh with 1,553,231 grid cells is considered fine enough and used for the numerical simulation study. In the coupled viscous and non-viscous solution method, the mesh independence study shows the 853,023 grid cells for viscous domain and 237,426 grid cells for non-viscous domain. All of these mesh sizes were optimally studied for the current data center model. In fact, the mesh size of the non-viscous domain is about 3.5 times fewer grid cells compared to the viscous domain.

\subsubsection{Results and Discussion}

\section{a. Whole Domain Results}

The standard $k-\varepsilon$ turbulence model with all of its high fidelity was chosen as the baseline case to be compared with other turbulence models. Fig. II.3 shows the temperature profiles of three other different turbulence models, i.e., the realizable $k-\varepsilon$ (Fig. II.3b), the SpalartAllmaras (Fig. II.3c), and the zero-equation models (Fig. II.3d). The temperature profile is 
sectioned at the middle plane in the y direction.

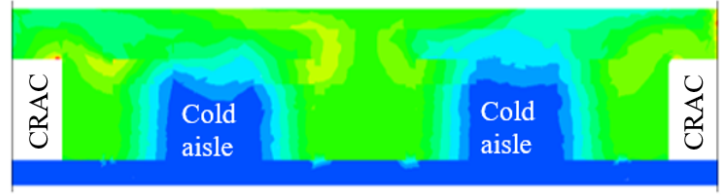

a) Standard $k-\varepsilon$ (baseline)

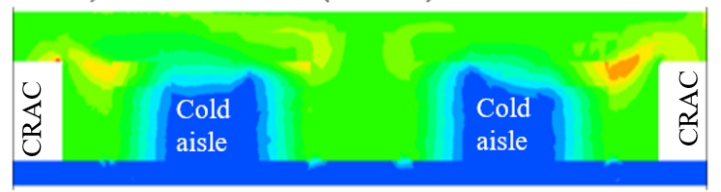

b) Spalart-Allmaras

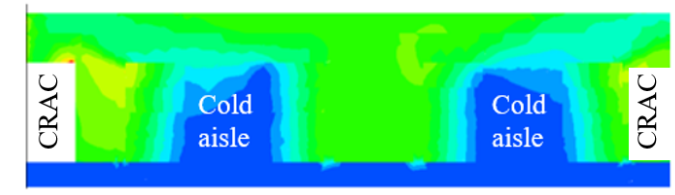

a) Realizable $k-\varepsilon$

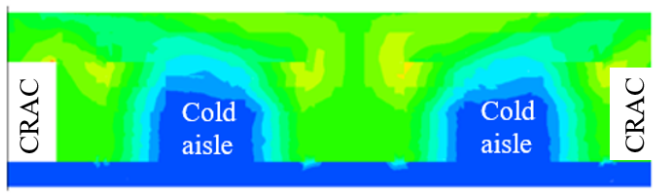

c) Zero-equation

Fig. II.3 Whole domain comparison for different turbulence models

It can be seen from Fig. II.3 that the zero-equation temperature profile most resembles the baseline case. The realizable $k$ - $\varepsilon$ and Spalart-Allmaras models spot some high-temperature areas, and therefore, result in larger differences. Table II. 1 clearly shows the absolute mean temperature difference defined by the average of the differences between other turbulence models compared to standard $k-\varepsilon$ model, and root mean square (RSM) difference. The zero-equation model has the smallest absolute mean temperature difference, and almost ties with the realizable $k-\varepsilon$ model. The $S$ - $A$ one-equation model experiences the largest temperature differences.

All the cases were run using the same personal Dell computer in our lab. The computer has $4 \mathrm{~GB}$ of memory, 4 cores with a clock speed of $3.30 \mathrm{GHz}$. The simulation time per 100 iterations and total solve time for all the cases run is presented in Table II.2. With the introduction of the hybrid method, one can see a significant reduction in computing time per hundred iterations. Indeed, it is seen that the hybrid method is approxmiate 2.17 times faster than the standard $k$ - $\varepsilon$, and about 1.91 times faster than the zero-equation model alone. 
The smaller mesh size in the non-viscous region along with the full mesh size of the viscous region makes up the total mesh size of the hybrid method which is approximately 1 million grid cells. In the case of the whole domain calculation, it is about 1.5 millions grid cells. The difference in mesh size accounts for the major reduction in computing time when comparing the hybrid method with the whole domain calculation.

Table II.1 Error table

\begin{tabular}{ccc}
\hline \multirow{2}{*}{ Other Turbulence Models } & Standard $\boldsymbol{k}-\boldsymbol{\varepsilon}$ Model \\
\cline { 2 - 3 } & Absolute Mean Temperature Difference (K) & RMSE (K) \\
\hline \hline Realizable $k-\varepsilon$ & 0.250 & 0.531 \\
Spalart - Allmaras & 0.373 & 0.782 \\
Zero - equation & 0.234 & 0.578 \\
\hline
\end{tabular}

Table II.2 Simulation time

\begin{tabular}{ccc}
\hline Turbulence Models & $\begin{array}{c}\text { Simulation Time (minutes) Per 100 } \\
\text { iterations }\end{array}$ & $\begin{array}{c}\text { Total Simulation Time } \\
\text { (hours) }\end{array}$ \\
\hline \hline Standard $k-\varepsilon$ (baseline) & $\sim 18.7$ & $\sim 6$ \\
Realizable $k-\varepsilon$ & $\sim 18.8$ & $\sim 4.8$ \\
Spalart - Allmaras & $\sim 19.3$ & $\sim 4$ \\
Zero - equation & $\sim 16.4$ & $\sim 2.45$ \\
Hybrid (Zero equation - & $\sim 8.58$ & $\sim 3.57$ \\
Standard $k-\varepsilon$ ) & & \\
\hline
\end{tabular}

Table II.3 The effect of inlet mass flow rate on viscous to non-viscous ratio

\begin{tabular}{cccc}
\hline $\begin{array}{c}\text { Inlet Mass Flow Rate } \\
(\mathbf{k g} / \mathbf{s})\end{array}$ & $\begin{array}{c}\text { Viscous Zone } \\
\text { Volume }\left(\mathbf{m}^{\mathbf{3}}\right)\end{array}$ & $\begin{array}{c}\text { Non-viscous Zone } \\
\text { Volume }\left(\mathbf{m}^{\mathbf{3}}\right)\end{array}$ & $\begin{array}{c}\text { Viscous to Non-Viscous } \\
\text { Ratio }\end{array}$ \\
\hline \hline 1 & 0 & 490 & 0 \\
3 & 1.92 & 488.1 & 0.004 \\
6 & 15.6 & 474.4 & 0.033 \\
9 & 54.6 & 435.4 & 0.1225 \\
12 & 113.2 & 376.8 & 0.300 \\
\hline
\end{tabular}




\section{b. Zone Partitioning Results}

Fig. II.4 shows the temperature profile of the middle plane of the partitioned viscous and non-viscous domains. The final combined picture (Fig. II.4c) of the zone partitioning solution is compared with the whole domain calculation using standard $k-\varepsilon$. (Fig. II.4d). The partitioning case over predicts the temperature in some areas, especially at the inlet to the CRAC unit and the region near the ceiling of the cold aisles.

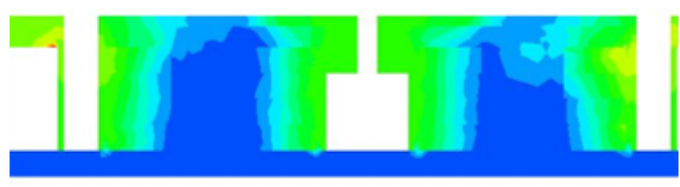

a) Viscous domain

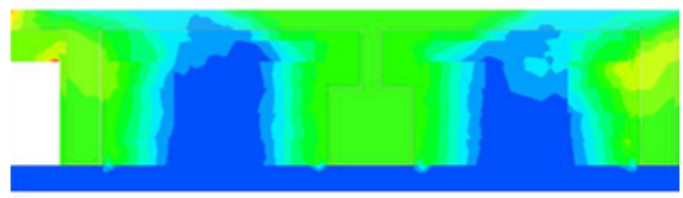

c) Combined viscous and non-viscous domains

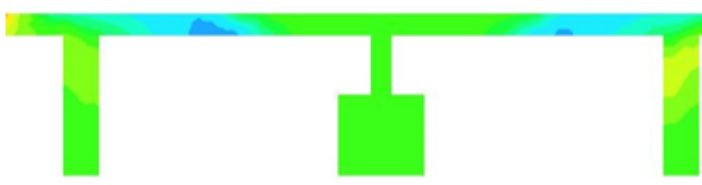

b) Non-viscous domain

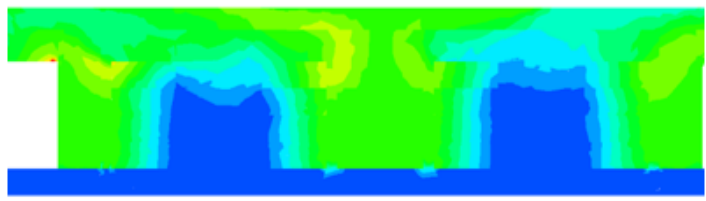

d) Whole viscous domain (baseline)

$\mathrm{T}\left({ }^{\circ} \mathrm{K}\right)$

290291292293294295296297298299300301302

Fig. II.4 Coupled viscous and non-viscous domains temperature profile

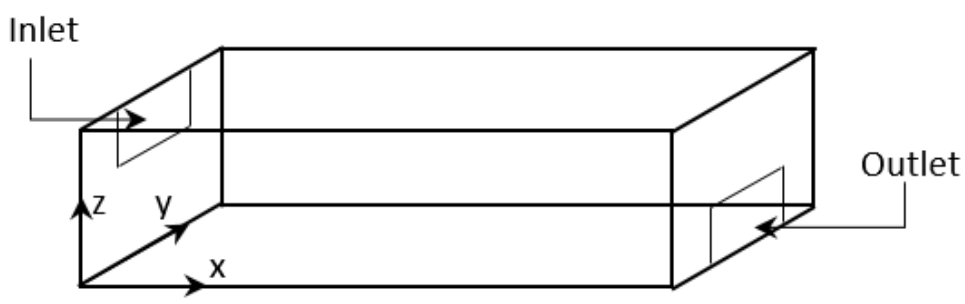

Fig. II.5 The simple box model 
The overall simulation time for combined viscous and non-viscous domains solution takes approximately 3.6 hours which is almost half the time of the whole domain calculation ( 6 hours).

\section{c. Limits of the Coupled Method}

In the partitioning method, the ratio of viscous to non-viscous domains has a significant impact on the speed of the entire simulation process. In fact, more volume of non-viscous over viscous domains offers a faster overall simulation time. Here, we investigate the effect of inlet velocity of the CRAC unit on the ratio of these two regions. In order to obtain this ratio, a simple box model is introduced. It is basically a rectangular box having the same size as the data center model with one inlet and one outlet as seen in Fig. II.5. The standard $k-\varepsilon$ turbulence model with the boundary conditions, i.e., mass flow inlet (inlet) and exhaust (outlet) are used.

Table II.3 shows the effects of different inlet mass flow rate on the viscous to non-viscous ratio. As we can see, the higher inlet mass flow rate results in an increased viscous - nonviscous ratio. No viscous zone associates with a mass flow of $1.0 \mathrm{~kg} / \mathrm{s}$, and the ratio is almost one-third as the highest mass flow rate observed. The closer to unity the ratio is, the less non-viscous region volume we have. Therefore, this suggests that the partitioning method will maximize the computational time at lower flow rate since more non-viscous regions present. As the ratio increases, the method is less attractive with growing viscous region size. 


\section{d. Verification of the Method Against a Two-Dimensional Test Case}

In this section, the coupled method is tested against a two-dimensional test case performed by Nielsen [51]. The geometry of the test room is presented in Fig. II.6. This test case can be used to test different two-dimensional CFD codes in the IEA Annex 20 work. The boundary conditions show that the model has one inlet where forced air is moved through an entrance near the ceiling of the chamber, whereas the outlet is located at the bottom near the floor of the opposite wall.

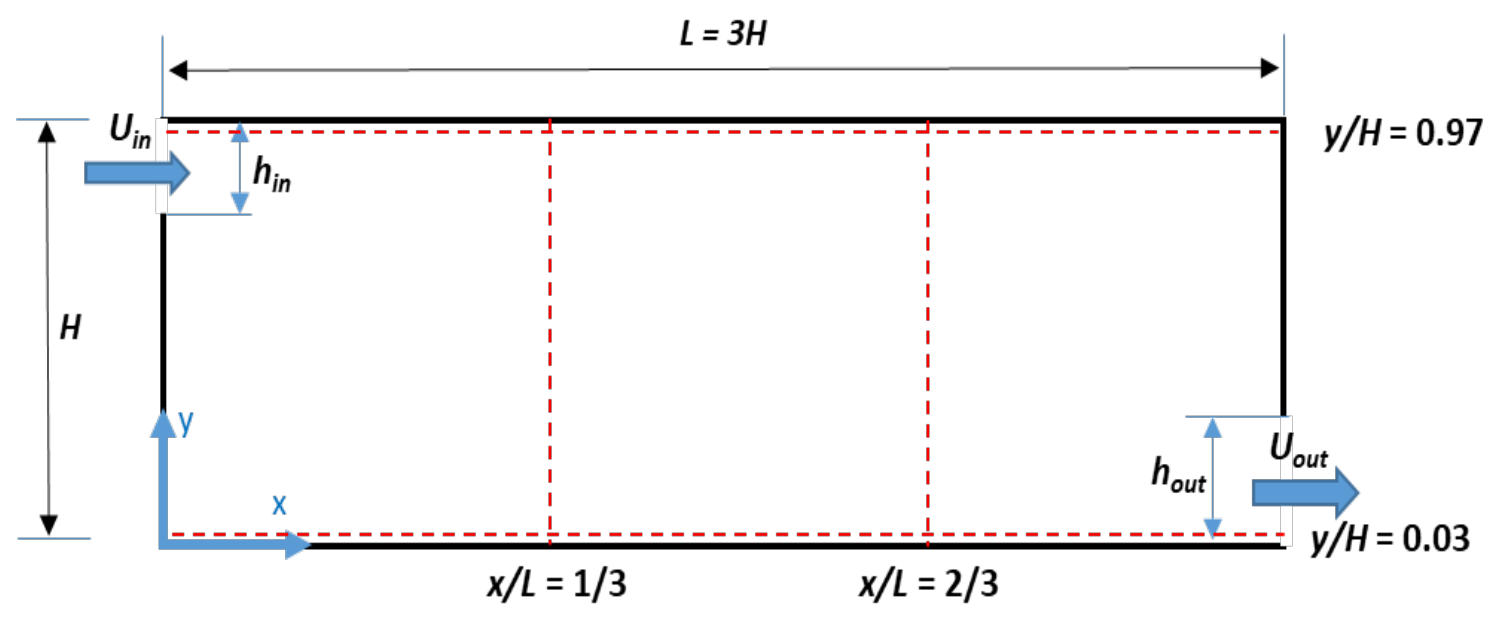

Fig. II.6 2D test case boundary conditions by Nielsen [51]

The total length $(L)$ of the chamber is equal three times the height $(H)$. There are totally four lines of measurement located at $x / L=1 / 3, x / L=2 / 3, y / H=0.03$, and $y / H=0.97$. All are represented as dotted lines in Fig. II.6.

The coupled method was carried out along with whole domain simulation using standard $k-\varepsilon$ turbulence model. The results of both methods are compared to the experimental results 
from four measuring lines outlined above. Fig. II.7 shows the comparison of the two methods against the experimental data by Nielsen [ref]. Overall, the coupled method can represent the trend of the experimental data although the whole domain simulation using standard $k-\varepsilon$ still shows superiority especially in the vertical testing regions.

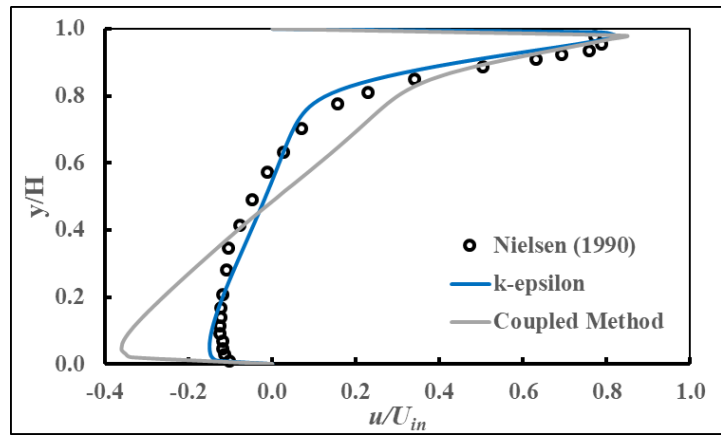

a) $x / L=1 / 3$

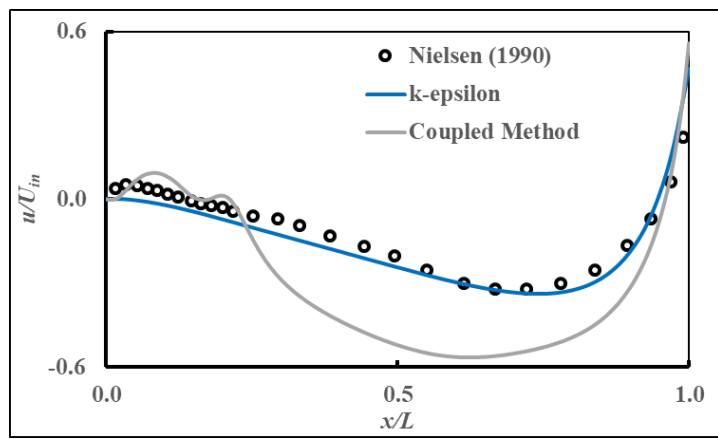

c) $\mathrm{y} / \mathrm{H}=0.03$

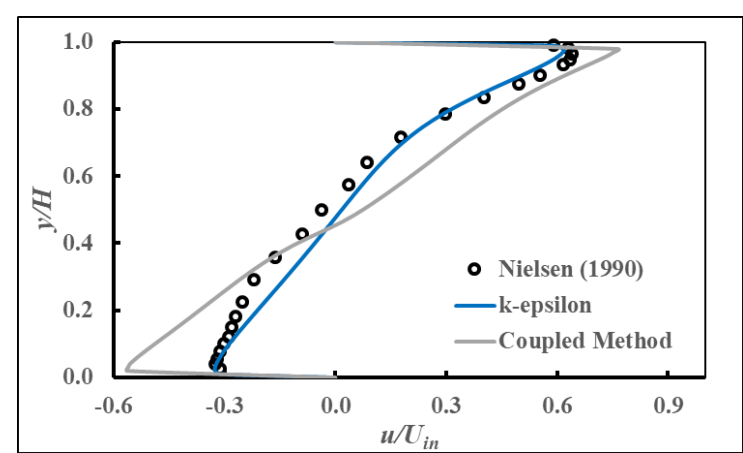

b) $x / L=2 / 3$

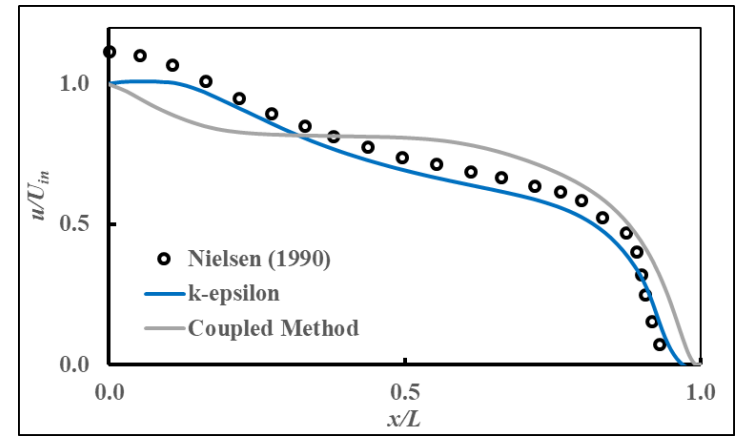

d) $\mathrm{y} / \mathrm{H}=0.97$

Fig. II.7 Reference velocity comparisons among the models

The actual root mean square error in Table II.5 indicates the errors associated with the two methods when compared against the experimental data. On average, the coupled method has about three times larger in error compared to the whole domain simulation method, however, it still shows very good match especially the ceiling region $\mathrm{y} / \mathrm{H}=0.97$ with only 
about half of the error compared to the whole domain simulation. Therefore, it is still a good potential to use the coupled method due to its great time reduction. Part of the errors quantified here is also due to the lack of better algorithm to divide the viscous and nonviscous regions more accurately. If this is done properly, the error for coupled method is expected to be less.

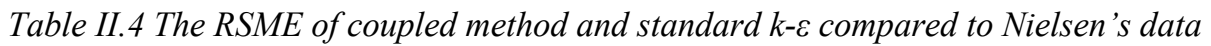

\begin{tabular}{|c|c|c|}
\hline \multicolumn{3}{|c|}{ RMSE $\left(\boldsymbol{U} / \boldsymbol{U}_{\text {in }}\right)$} \\
\hline & Coupled Method & Standard $k-\varepsilon$ \\
\hline$x / L=3$ & 0.14 & 0.06 \\
\hline$x / L=2 / 3$ & 0.15 & 0.04 \\
\hline$y / H=0.03$ & 0.17 & 0.05 \\
\hline$y / h=0.97$ & 0.17 & 0.09 \\
\hline
\end{tabular}

\subsection{Evaluation of Turbulence and Tile Modeling}

In this section, a detailed validation study of ten turbulence models is investigated, i.e., Chen-Xu zero equation [13], Spalart-Allmaras one equation [68], standard $k-\varepsilon$ [44], realizable $k-\varepsilon$ [64], RNG $k-\varepsilon$ [56], low Reynolds number $k-\varepsilon$ (LRN k-E) [43], standard $k-\omega$

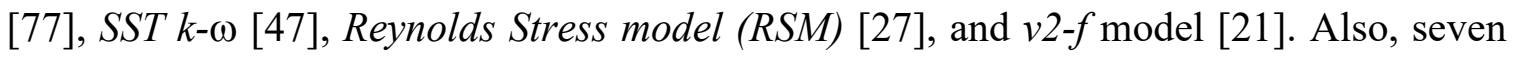
tile models are studied and compared, i.e., detailed [8], single opening, fully opening [36], [1], [2], [3] porous media, porous jump [8], [7], [9] body force [1], [2], [3] and modified body models [8], [7], [9]. Porous media is the newly added tile model along with zeroequation, one-equation, $L R N k-\varepsilon$, standard $k-\omega, S S T k-\omega, R S M$, and $v 2-f$ turbulence models that will be investigated for the first time in this study. The models at three tile-to-server airflow ratios, $20 \%, 60 \%$, and $100 \%$, are assessed, and recommendations on tile and turbulence models are discussed. A sensitivity study of different tile and turbulence model 
combinations is carefully inspected. Finally, an overall performance index is proposed for each turbulence and title model relative to the baseline model. Top models are recommended based on the targeting criteria in computing time and accuracy.

\subsubsection{CFD Modeling}

\section{a. Experimental Model Description}

The single-server rack's physical model in a data center as seen in Fig. II.8 has been investigated by previous experimental studies [41], [9]. The test data provides a comprehensive velocity information suited for the evaluation study of the current study. Arghode et al. [9] have studied this server rack model by comparing various tile models and validate them against the experimental result. Only one server rack in a data center laboratory is studied. The standard $42 \mathrm{U}$ server rack is filled with four $10 \mathrm{U}$ rack-mounted server simulators. Each server simulator model has a dimension of 24 in. $(60.96 \mathrm{~cm})$ wide, 18 in. $(45.72 \mathrm{~cm})$ high, and 27.125 in. $(69 \mathrm{~cm})$ deep. There is a 6 in. $(15.24 \mathrm{~cm})$ gap at the foot of the server rack. There are 4 fans mounted on a plate located $10 \mathrm{in} .(25.4 \mathrm{~cm})$ away from the server front of each server. The fans are cylindrical with a depth of 2 in. $(5.1 \mathrm{~cm})$. The perforated tile and the dampers underneath have a distance of $1.5 \mathrm{in} .(3.81 \mathrm{~cm})$ away from each other. Both contribute to an equivalent porosity of $31 \%$, whereas the server inlet which consists of plastic grilles has an equivalent porosity of $33 \%$. The raised floor is relatively deep ( $3 \mathrm{ft}$ or $0.9 \mathrm{~m}$ ) compared to a 2 -foot raised floor of industry standard. The probing line where measured velocity points are taken place is also shown in Fig. II.8 

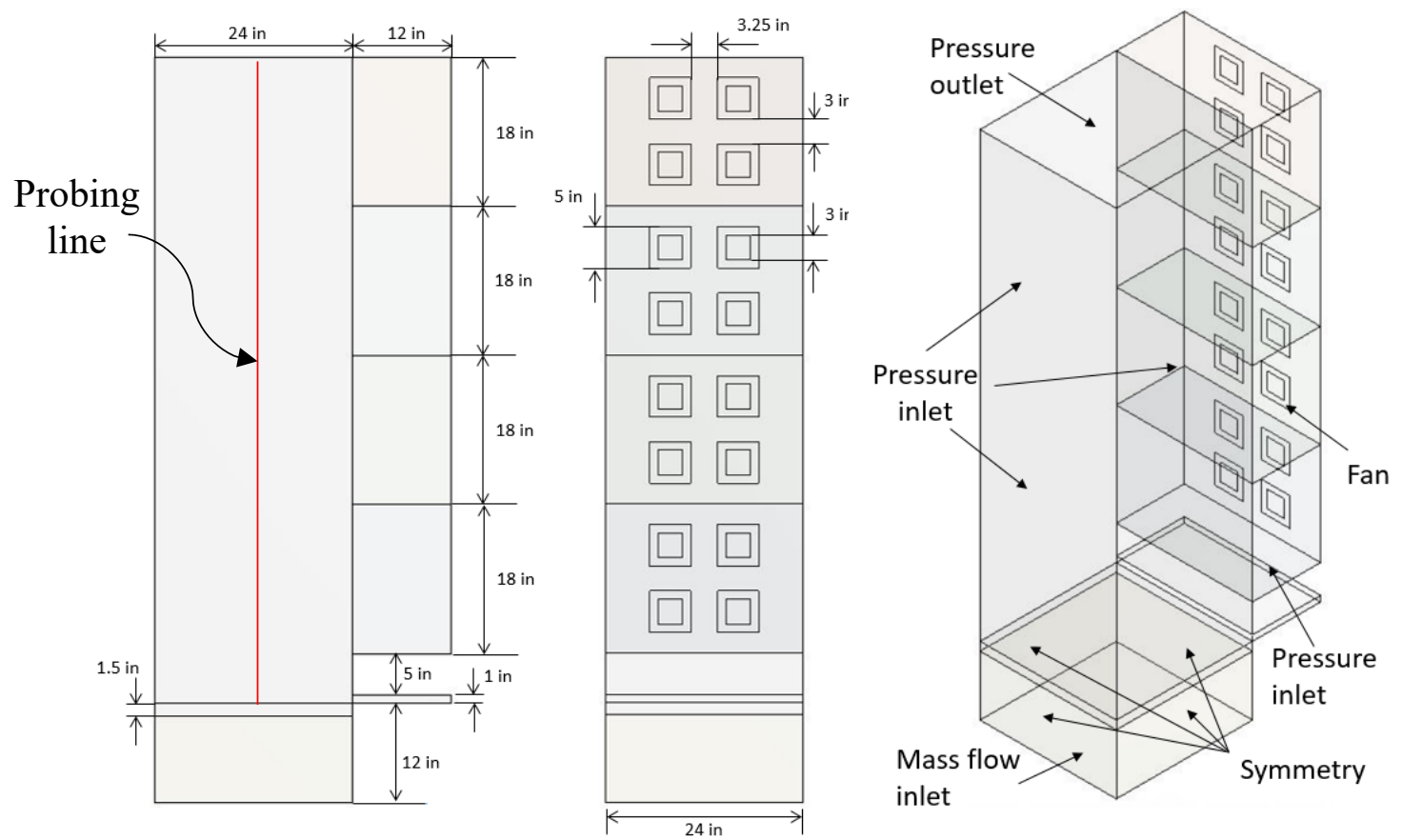

Fig. II.8 Server rack model: a) geometrical dimensions b) boundary conditions.

\section{b. Computational Model}

The computational fluid dynamics domain of the current server model is shown in Fig. II.8b. The room air positioned on top of the tile and in front of the rack inlet is included in the model. Three faces of the room air are specified as pressure inlet, and only the top surface is specified as pressure outlet. Since the server simulator is modeled inside a data center lab along with other real server racks and only one server rack is considered in this study, the symmetry condition is imposed at the sides of the raised floor. In addition, only a third of the raised floor ( $12 \mathrm{in}$ or $30.48 \mathrm{~cm}$ deep) is modeled due to its fairly deep aspect. Hence, the bottom surface of the raised floor is specified as mass flow inlet normal to the surface without any directional flows. In addition, only a portion inside the servers are modeled. That is, only the spaces between the rack inlet and the fan plate are considered in the computational domain. Although the fans are cylindrical, their modeling is a 
representation of square fan hubs for simplicity. The fan boundary condition is specified as exhaust fans. The little gap between the floor and the foot of the server rack is specified as pressure inlet.

Since the server rack model consists of mostly orthogonal features, it is therefore meshed with a cut-cell method where hexahedral mesh is dominant. Grid independence study is performed to ensure for an accurate result yet less computational effort. Initially, the rack model is meshed with a coarse mesh which results in approximately 125,000 cells, the mesh size is gradually increased to see how it affects the velocity profile as seen in Fig. II.9. The mesh regions near the perforated tile, server rack inlet grilles, and exhaust fans are enhanced with respect to a higher mesh density due to their important physics.

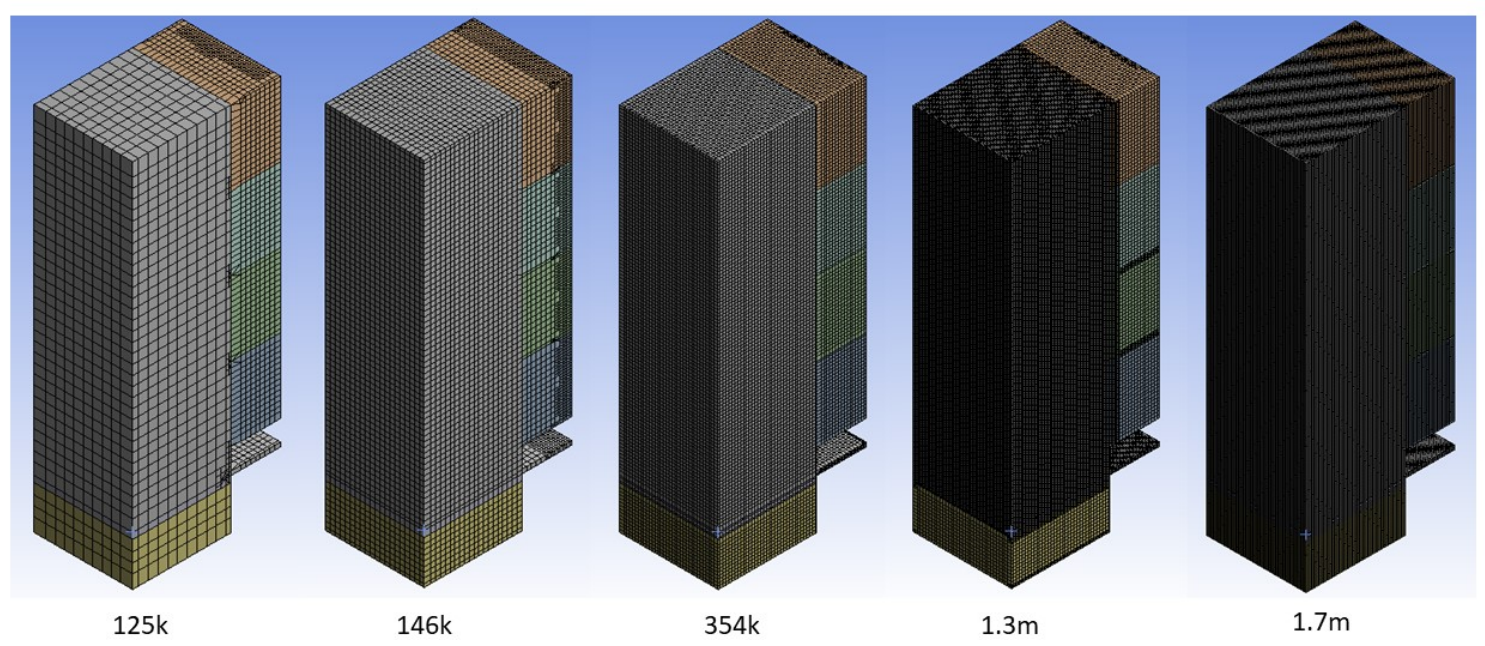

Fig. II.9 Mesh representations at various cell sizes

It is finally optimized at 1.3 million cells because further increase in mesh size shows little 
change in the after-tile air flow velocities yet adding more computational cost. The typical velocities along the probing line located at the center of the perforated tile for different mesh sizes are shown in Fig. II.10. The 1.3-million-cell line is almost overlapping the 1.7million-cell line. A similar trend is also reflected when examining the flow streams at the middle plane right in front of the server rack inlet. The last two final mesh sizes show almost identical flow streams when comparing side by side with others. (See Fig. II.11).

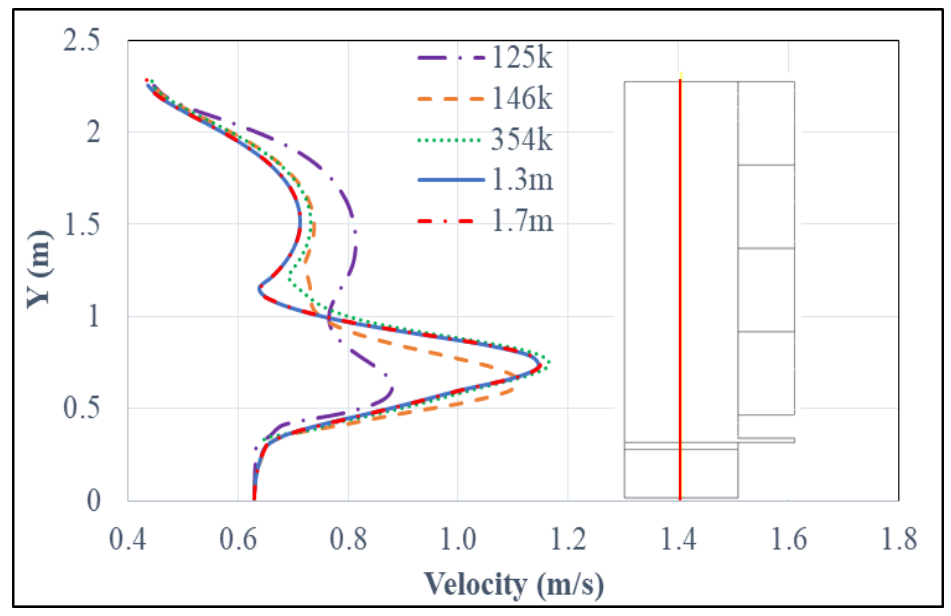

Fig. II.10 Velocities along the probing line for different mesh sizes

Table II.5 Simulation time for different mesh sizes

\begin{tabular}{|c|c|c|c|c|}
\hline $\begin{array}{c}\text { Mesh } \\
\text { Size }\end{array}$ & $\begin{array}{c}\text { Time per } \\
\text { Iteration } \\
\text { (seconds) }\end{array}$ & $\begin{array}{c}\text { Number of } \\
\text { Iterations }\end{array}$ & $\begin{array}{c}\text { Total Wall Clock } \\
\text { Time (seconds) }\end{array}$ & $\begin{array}{c}\text { Total Wall Clock } \\
\text { Time (minutes) }\end{array}$ \\
\hline $125 \mathrm{k}$ & 0.266 & 996 & 265.289 & 4.42 \\
\hline $146 \mathrm{k}$ & 0.332 & 2217 & 735.299 & 12.3 \\
\hline $354 \mathrm{k}$ & 0.810 & 4363 & 3535.132 & 58.9 \\
\hline $1.3 \mathrm{~m}$ & 3.181 & 3503 & 11142.102 & 185.7 \\
\hline $1.7 \mathrm{~m}$ & 4.217 & 4348 & 18337.653 & 305.6 \\
\hline
\end{tabular}


The analyses were run using 7 cores from a personal Dell computer Intel ${ }^{\circledR}$ Core $^{\mathrm{TM}} \mathrm{i} 7-4770$ CPU at $3.4 \mathrm{GHz}$ with $32 \mathrm{~GB}$ of memory.

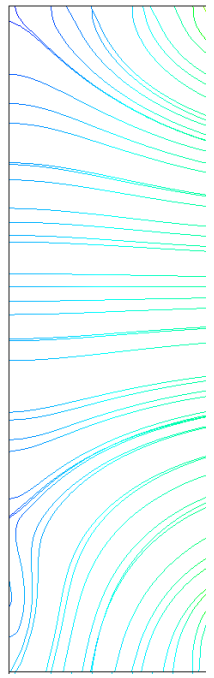

$125 \mathrm{k}$

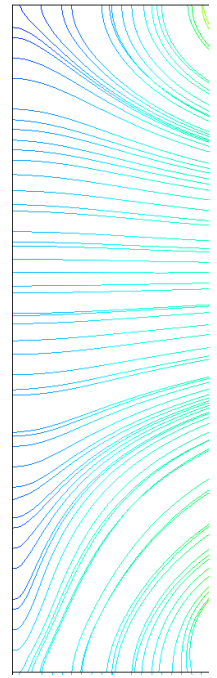

$146 \mathrm{k}$

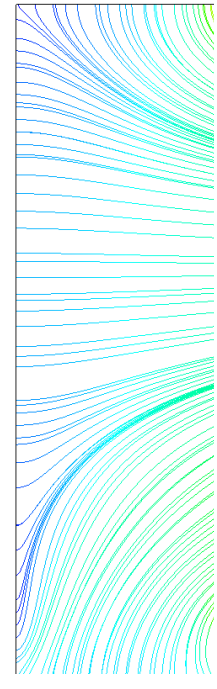

$354 \mathrm{k}$

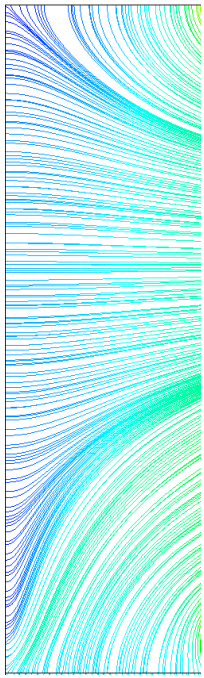

$1.3 \mathrm{~m}$

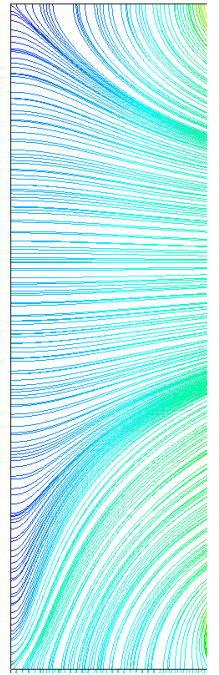

$1.7 \mathrm{~m}$

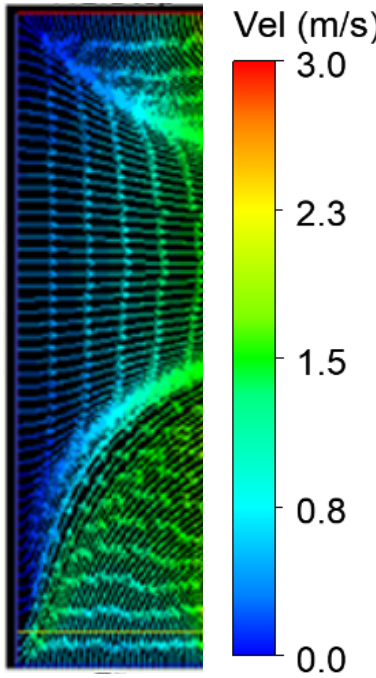

Exp.

Fig. II.11 Flow streams at various mesh sizes

\section{c. Numerical Model}

The convergence criteria are set to be less than $10^{-4}$ in residuals for all computed variables. all computed variables. In some cases where set criteria cannot be reached, the quantity of interests are monitored to stabilize to a constant value within less than $1 \%$ of a difference for a convergence to be reached. The gradients in spatial discretization are selected as least square cell based. The pressure-velocity coupling scheme used is the SIMPLE algorithm. Turbulent kinetic energy and turbulent dissipation rate equations utilize the first order 
upwind scheme, while the momentum equation is used with the second order upwind scheme.

\subsubsection{Tile Modeling}

\section{a. Detailed Model}

In this model, every feature is modeled explicitly, i.e., the tile pores, linear grilles at the inlet of the server rack, the tile damper as seen in Fig. II. 12 a. All features are modeled as orthogonal shapes. The detailed modeled is then simulated with appropriate boundary conditions that is outlined in Sect. 3.2.1b.

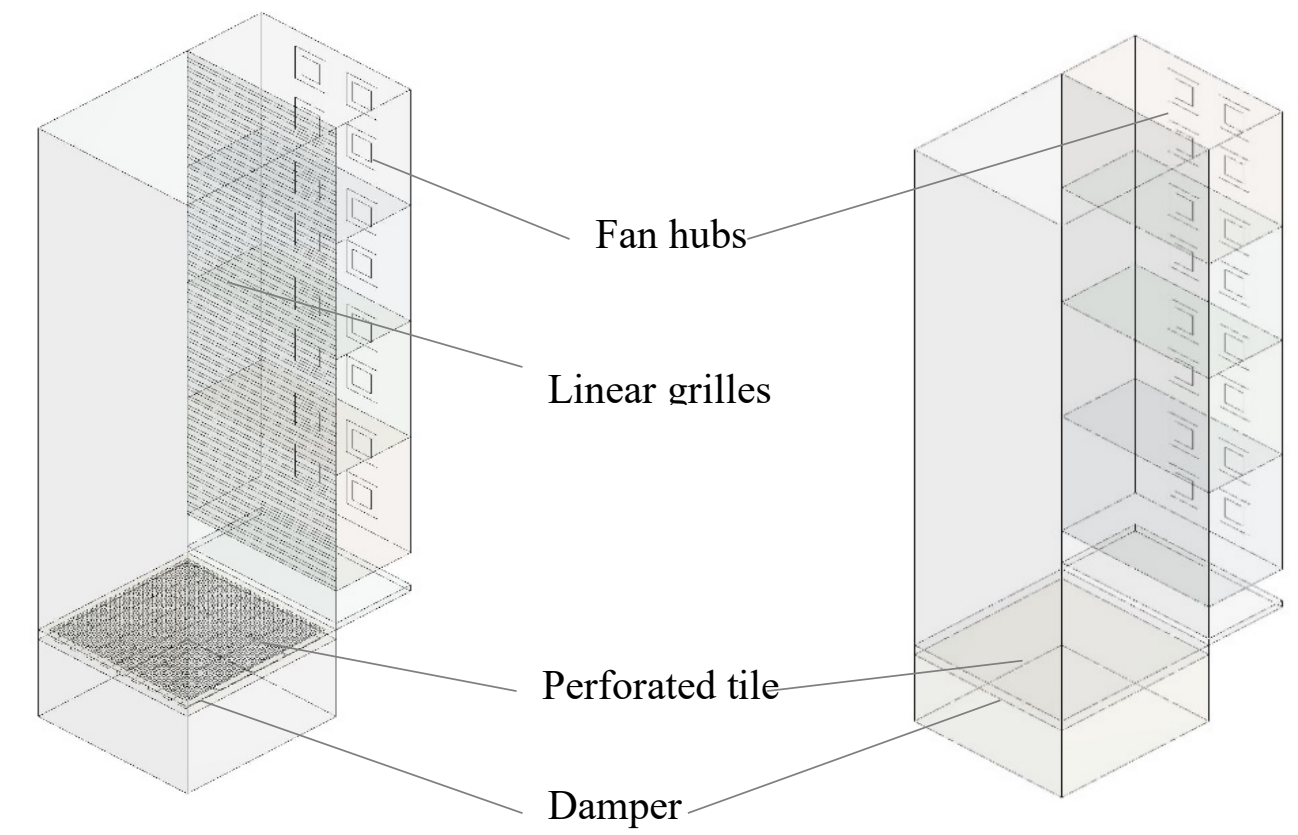
a) Detailed geometry
b) Simplified geometry

Fig. II.12 One server rack model: detailed vs. simplified 


\section{b. Single Opening}

In this model, the objective is to find the flow area through a simple formulation as outlined in Eq. (II.11). The actual flow area $\left(A_{\text {flow }}\right)$ is calculated by multiplying the tile area $\left(A_{\text {tile }}\right)$ which is typically $4 \mathrm{ft}^{2}$ with the effective porosity $\left(F_{e f f}\right)$. The challenge part is to tune the effective porosity so that it matches the experimental data. The search range for the effective porosity is between the actual perforation and unity $\left(F \leq F_{\text {eff }} \leq 1\right)$. Sometimes it takes many trials and errors to optimize the most suitable effective porosity.

$$
A_{\text {flow }}=F_{\text {eff }} \times A_{\text {tile }}
$$

\section{c. Fully Open}

The fully open model is essentially derived from the single opening model by taking the effective porosity equal to unity $\left(F_{\text {eff }}=1.0\right)$. The flow area is now the actual tile area. This model is a very crude tile model and often effective for tiles with high perforation $(F>$ $50 \%)$. However, it becomes a problem when dealing with tiles with low perforation $(F<$ $50 \%)[2]$.

\section{d. Porous Jump}

In the PJ model, a step pressure loss across the tiles are specified. This pressure loss is calculated using Eq. (II.12), while the pressure loss factor can be referred to Eq. (II.13). 


$$
\begin{gathered}
\Delta p=K \frac{1}{2} \rho V_{i n}^{2} \\
K=\frac{1}{F^{2}}\left(\left(\frac{1-F}{2}\right)^{2}+(1-F)\right)^{2}
\end{gathered}
$$

$K=$ pressure loss factor

$F=$ porosity

$V_{\text {in }}=$ velocity before the tiles

$\Delta p=$ pressure drop across tiles

$\rho=$ air density

The PJ model only specifies a step pressure loss and has little effects on the downstream velocity after tiles. In reality, the airflow through tiles also experiences an air momentum boost after flowing through the pores. Because the PJ model does not capture this feature, a BF model is introduced to account for this momentum increase.

\section{e. Porous Media}

The porous media model is a three-dimensional version of the one-dimensional porous jump model. In the porous media model, a momentum source term is added to the standard fluid flow equations. The viscous and inertial loss terms are the two parts of this source term. When modeling a perforated plate, the permeability term is normally eliminated and only the inertial loss term is used, yielding the following simplified form of the porous media equation (Eq. (II.14)).

$$
\nabla p=-\sum_{j=1}^{3} C_{2 i j}\left(\frac{1}{2} \rho v_{j}|v|\right)
$$


$\nabla p=$ pressure gradient

$C_{2 i j}=$ prescribed matrix, becomes $C_{2}$ (inertial resistance factor) in the case of simple homogeneous porous media

$\rho=$ air density

$|v|=$ magnitude of the velocity

\section{f. Body Force Model}

In the BF model, a Y-momentum source is specified above the tile surface to account for the acceleration of air after passing through tile pores. The Y-direction is normal to the surface of the tiles. Eq. (II.15) - (II.17) show the formulation of this momentum source.

$$
\begin{gathered}
S_{y}=\frac{\Delta M}{\forall} \\
\Delta M=\rho A_{\text {tile }} V_{\text {in }}\left(V_{\text {pore }}-V_{\text {in }}\right) \\
V_{\text {pore }}=\frac{V_{\text {in }}}{F}
\end{gathered}
$$

$S_{y}=$ momentum source in the y-direction, $\Delta M=$ momentum gain across tiles, $A_{\text {tile }}=$ tile area, $V_{\text {in }}=$ velocity before the tiles, $V_{\text {pore }}=$ pore velocity after the tiles, $F=$ porosity In this BF model, volume of the momentum source region $(\forall)$ is located right above the tiles [1], [2], [3], [8]. The height of the momentum source region is taken as $0.0102 \mathrm{~m}$ (4 in.), while the base area matches the tile area which is $0.61 \times 0.61 \mathrm{~m}(2 \mathrm{ft} . \times 2 \mathrm{ft}$.). Step pressure loss across the tiles can be specified similar to the PJ model. 


\section{g. Modified Body Force Model}

There is a suspect that even higher momentum along the flow path from tile surface exists. Therefore, a modified body force (MBF) model is created to correct this momentum deficit [8]. In the MBF model, the Y-momentum source is specified above the tile surface similar to the BF model, however, the velocity at the vena contracta Eq. (II.20) is used instead of the pore velocity in the model. This gives a higher $\mathrm{Y}$-momentum flow rate as compared to the BF model. The formulation of this modified momentum source can be referred in Eq. (II.18) - (II.20).

$$
\begin{gathered}
S_{y}=\frac{\Delta M}{\forall} \\
\Delta M=\rho A_{\text {tile }} V_{\text {in }}\left(V_{\text {neck }}-V_{\text {in }}\right) \\
V_{\text {neck }}=V_{\text {in }}(K+1)^{\frac{1}{2}}
\end{gathered}
$$

$V_{\text {neck }}=$ the vena contracta velocity after the tiles, $V_{\text {in }}=$ velocity before the tiles, $K=$ pressure loss factor, $S_{y}=$ momentum source in the y-direction, $\Delta M=$ momentum gain across tiles, $A_{\text {tile }}=$ tile area. With the use of a higher velocity in the model, the momentum source now has an even more profound effect in the downstream of the flow stream. The base area of the momentum source region is taken as the tile area $0.61 \times 0.61 \mathrm{~m}(2 \mathrm{ft} . \mathrm{x} 2 \mathrm{ft}$. $)$, while the height of the momentum source is taken as $0.0102 \mathrm{~m}$ (4 in.). Also, the step pressure is also maintained as the same as specified in the PJ model. 


\subsubsection{RANS Turbulence Modeling}

\section{a. Zero Equation Model}

The zero equation consists of a simple algebraic equation for turbulence viscosity and no other partial different equations is used. It was originally developed by Prandtl back in 1925. Although the model does not strongly hold in many diverse applications and prone to large errors with complex geometries, it is useful in some carefully calibrated models of specific types of flow. When it comes to indoor air environments, $\mathrm{Chen}$ and $\mathrm{Xu}$ [13] has modified the zero equation to better adapt to the characteristics of enclosure settings. The algebraic formulation is derived to express turbulent viscosity as follows,

$$
v_{t}=0.3874 U L
$$

where $U$ is the local mean and $L$ is the nearest wall distance. The proposed empirical constant is 0.03874 for different flows. The model has been extensively validated by many different parties including the original authors, Chen and $\mathrm{Xu}$. The model has been used for

simulating airflows in different indoor environments with acceptable accuracy and significant time reduction. Therefore, it is included in this data center study to assess and evaluate with other advanced turbulence models for data centers.

\section{b. One-Equation Model}

The one-equation model by Spalart-Allmaras (S-A) [68] differs from other one-equation models by directly solving for the kinematic eddy viscosity, $\tilde{v}$, 


$$
\frac{\partial}{\partial t}(\rho \tilde{v})+\frac{\partial}{\partial x_{i}}\left(\rho \tilde{v} u_{i}\right)=G_{v}+\frac{1}{\sigma_{\tilde{v}}}\left[\frac{\partial}{\partial x_{j}}\left\{(\mu+\rho \tilde{v}) \frac{\partial \tilde{v}}{\partial x_{j}}\right\}+C_{b 2} \rho\left(\frac{\partial \tilde{v}}{\partial x_{j}}\right)^{2}\right]-Y_{v}+S_{\tilde{v}}
$$

The S-A model is advantageous for various grids of any structures since its solution is solved locally at each point and independent of other neighboring points. Although the SA model was designed for wall-bounded flows and boundary layers applications subjected to adverse pressure gradients, it is also reliable in the indoor environment simulation compared to other one-equation models [57].

\section{c. Standard $k-\varepsilon$ Model}

Standard $k-\varepsilon$ model proposed by Launder and Spalding [44] is the most prevalent in the $k$ $\varepsilon$ two-equation model family. The two transport equations for kinetic energy $(k)$ and dissipation rate $(\varepsilon)$ are shown below.

$$
\begin{gathered}
\frac{\partial}{\partial t}(\rho k)+\frac{\partial}{\partial x_{i}}\left(\rho k u_{i}\right)=\frac{\partial}{\partial x_{j}}\left[\left(\mu+\frac{\mu_{t}}{\sigma_{k}}\right) \frac{\partial k}{\partial x_{j}}\right]+G_{k}+G_{b}-\rho \varepsilon-Y_{M}+S_{k} \\
\frac{\partial}{\partial t}(\rho \varepsilon)+\frac{\partial}{\partial x_{i}}\left(\rho \varepsilon u_{i}\right)=\frac{\partial}{\partial x_{j}}\left[\left(\mu+\frac{\mu_{t}}{\sigma_{\varepsilon}}\right) \frac{\partial \varepsilon}{\partial x_{j}}\right]+C_{1 \varepsilon} \frac{\varepsilon}{k}\left(G_{k}+C_{3 \varepsilon} G_{b}\right)-C_{2 \varepsilon} \rho \frac{\varepsilon^{2}}{k}+S_{\varepsilon}
\end{gathered}
$$

This turbulence model is widely accepted in the industry because of numerous validations.

Therefore, it is popularly used to simulate indoor environments. Many researchers from the literature is reportedly used this turbulence for data center studies. [8] [7], [9], [14], [36], [70], [86], [81]. 


\section{d. RNG $k-\varepsilon$ Model}

The $R N G k-\varepsilon$ model proposed by Yakhot and Orszag [56] was derived following the renormalization group $(R N G)$ theory. The two transport equations are as follows,

$$
\begin{gathered}
\frac{\partial}{\partial t}(\rho k)+\frac{\partial}{\partial x_{i}}\left(\rho k u_{i}\right)=\frac{\partial}{\partial x_{j}}\left[\alpha_{k} \mu_{e f f} \frac{\partial k}{\partial x_{j}}\right]+G_{k}+G_{b}-\rho \varepsilon-Y_{M}+S_{k} \\
\frac{\partial}{\partial t}(\rho \varepsilon)+\frac{\partial}{\partial x_{i}}\left(\rho \varepsilon u_{i}\right)=\frac{\partial}{\partial x_{j}}\left[\alpha_{\varepsilon} \mu_{e f f} \frac{\partial \varepsilon}{\partial x_{j}}\right]+C_{1 \varepsilon} \frac{\varepsilon}{k}\left(G_{k}+C_{3 \varepsilon} G_{b}\right)-C_{2 \varepsilon} \rho \frac{\varepsilon^{2}}{k}-R_{\varepsilon}+S_{\varepsilon}
\end{gathered}
$$

The numerous refinements of the $R N G k-\varepsilon$ over the standard $k-\varepsilon$ make the model good for high streamline curvature and strain rate, transitional flows, and wall heat and mass transfer. Details of each term notation for Eqs. (II.25), (II.26) can be found in Appendix A Table A.1.

\section{e. Realizable k- $\varepsilon$ Model}

The model realizable $k-\varepsilon$ by Shih et al [64] adds a new modified model equation for dissipation $(\varepsilon)$ (Eq. (II.28)). The two transport equations for the model are as follows,

$$
\begin{gathered}
\frac{\partial}{\partial t}(\rho k)+\frac{\partial}{\partial x_{i}}\left(\rho k u_{i}\right)=\frac{\partial}{\partial x_{j}}\left[\left(\mu+\frac{\mu_{t}}{\sigma_{k}}\right) \frac{\partial k}{\partial x_{j}}\right]+G_{k}+G_{b}-\rho \varepsilon-Y_{M}+S_{k} \\
\frac{\partial}{\partial t}(\rho \varepsilon)+\frac{\partial}{\partial x_{i}}\left(\rho \varepsilon u_{i}\right)=\frac{\partial}{\partial x_{j}}\left[\left(\mu+\frac{\mu_{t}}{\sigma_{\varepsilon}}\right) \frac{\partial \varepsilon}{\partial x_{j}}\right]+\rho C_{1} S \varepsilon-\rho C_{2} \frac{\varepsilon^{2}}{k+\sqrt{v \varepsilon}}+C_{1 \varepsilon} \frac{\varepsilon}{k} C_{3 \varepsilon} G_{b}+S_{\varepsilon}
\end{gathered}
$$

With these modifications, the realizable model shows the better performance compared to all $k-\varepsilon$ models for several applications of separated flows and complex secondary flows. The details of each term notation for Eqs. (II.27), (II.28) can be found in Appendix A Table A.1. 


\section{f. Low Reynolds Number $k-\varepsilon$ Model}

Low Reynolds number $(L R N)$ turbulence models are good remedies in for near-wall treatment. These models normally require a very fine grid near the walls, which demands a higher computing time. Although there are many $L R N$ models have been proposed, they are of similar forms. The $L R N k-\varepsilon$ model proposed by Launder and Sharma [43] is the one chosen in this study. The two transport equations for this model are revealed in Eqs. (II.29), (II.30).

$$
\begin{gathered}
\frac{\partial}{\partial t}(\rho k)+\frac{\partial}{\partial i}\left(\rho k u_{i}\right)=\frac{\partial}{\partial x_{j}}\left[\left(\mu+\frac{\mu_{t}}{\sigma_{k}}\right) \frac{\partial k}{\partial x_{j}}\right]+G_{k}+G_{b}-\rho \varepsilon-Y_{M}+D+S_{k} \\
\frac{\partial}{\partial t}(\rho \varepsilon)+\frac{\partial}{\partial x_{i}}\left(\rho \varepsilon u_{i}\right)=\frac{\partial}{\partial x_{j}}\left[\left(\mu+\frac{\mu_{t}}{\sigma_{\varepsilon}}\right) \frac{\partial \varepsilon}{\partial x_{j}}\right]+\frac{C_{\varepsilon 1}^{*} G_{k} \varepsilon}{k}-\frac{C_{\varepsilon 2}^{*} \rho \varepsilon^{2}}{k}+E+S_{\varepsilon}
\end{gathered}
$$

Although $L R N$ models are useful in some specific situations where information near-wall regions are significant, they remain costly in terms of computing time and may not improve model accuracy very much. Therefore, their applications on indoor simulations are still limited due to the lack of applicability.

\section{g. Standard $k$ - $\omega$ Model}

The standard $k-\omega$ model is originally proposed by Wilcox [77]. The standard $k-\omega$ model is established based on two model transport equations, i.e., the turbulence kinetic energy

(k) and the specific dissipation rate $(\omega)$ which is the ratio of $\varepsilon$ to $k$. The two transport equations for $k$ and $\omega$ are shown in Eqs. (II.31), (II.32),

$$
\frac{\partial}{\partial t}(\rho k)+\frac{\partial}{\partial x_{i}}\left(\rho k u_{i}\right)=\frac{\partial}{\partial x_{j}}\left[\Gamma_{k} \frac{\partial k}{\partial x_{j}}\right]+G_{k}-Y_{k}+S_{k}
$$




$$
\frac{\partial}{\partial t}(\rho \omega)+\frac{\partial}{\partial x_{i}}\left(\rho \omega u_{i}\right)=\frac{\partial}{\partial x_{j}}\left[\Gamma_{\omega} \frac{\partial \omega}{\partial x_{j}}\right]+G_{\omega}-Y_{\omega}+S_{\omega}
$$

To improve the accuracy of the $k-\omega$ model, production terms are normally added to both the $k$ and $\omega$ equations, which shows advantages for predicting free shear flows. The $k-\omega$ model has recently been used to predict the indoor air environment simulations.

\section{h. SST $k-\omega$ Model}

The main problem with the Wilcox model [77] is its well-known strong sensitivity to freestream conditions. Menter [47] developed the SST $k$ - $\omega$ model by using a blending function to effectively use in the near-wall region with the freestream in the far field. The two transport equations of the SST $k$ - $\omega$ model are shown in Eqs. (II.33), (II.34).

$$
\begin{gathered}
\frac{\partial}{\partial t}(\rho k)+\frac{\partial}{\partial x_{i}}\left(\rho k u_{i}\right)=\frac{\partial}{\partial x_{j}}\left[\Gamma_{k} \frac{\partial k}{\partial x_{j}}\right]+G_{k}-Y_{k}+S_{k} \\
\frac{\partial}{\partial t}(\rho \omega)+\frac{\partial}{\partial x_{i}}\left(\rho \omega u_{i}\right)=\frac{\partial}{\partial x_{j}}\left[\Gamma_{\omega} \frac{\partial \omega}{\partial x_{j}}\right]+G_{\omega}-Y_{\omega}+D_{\omega}+S_{\omega}
\end{gathered}
$$

The flexibility of the SST $k$ - $\omega$ model in the near-wall and far-field regions shows good accuracy and reliability for a wider range of flows compared to the standard $k-\omega$ model. One of these flows is the adverse pressure gradient flows.

\section{i. v2-f Model}

The model $(V 2 F)$, based on Durbin's $k-\varepsilon-v^{2}$ model [21] incorporates near-wall turbulence anisotropy and non-local pressure-strain effects added to the standard $k-\varepsilon$ model. The 
model has four transport equations for the turbulence kinetic energy $(k)$, its dissipation rate $(\varepsilon)$, a velocity variance scale $\left(\overline{v^{2}}\right)$, and an elliptic relaxation function $(f)$.

$$
\begin{gathered}
\frac{\partial}{\partial t}(\rho k)+\frac{\partial}{\partial x_{i}}\left(\rho k u_{i}\right)=P-\rho \varepsilon+\frac{\partial}{\partial x_{j}}\left[\left(\mu+\frac{\mu_{t}}{\sigma_{k}}\right) \frac{\partial k}{\partial x_{j}}\right]+S_{k} \\
\frac{\partial}{\partial t}(\rho \varepsilon)+\frac{\partial}{\partial x_{i}}\left(\rho \varepsilon u_{i}\right)=\frac{C_{\varepsilon 1}^{\prime} P-C_{2 \varepsilon} \rho \varepsilon}{T}+\frac{\partial}{\partial x_{j}}\left[\left(\mu+\frac{\mu_{t}}{\sigma_{\varepsilon}}\right) \frac{\partial \varepsilon}{\partial x_{j}}\right]+S_{\varepsilon} \\
\frac{\partial}{\partial t}\left(\rho \overline{v^{2}}\right)+\frac{\partial}{\partial x_{i}}\left(\rho \overline{v^{2}} u_{i}\right)=\rho k f-6 \rho \overline{v^{2}} \frac{\varepsilon}{k}+\frac{\partial}{\partial x_{j}}\left[\left(\mu+\frac{\mu_{t}}{\sigma_{k}}\right) \frac{\partial \overline{v^{2}}}{\partial x_{j}}\right]+S_{\overline{v^{2}}} \\
f-L^{2} \frac{\partial^{2} f}{\partial x_{j}^{2}}=\left(C_{1}-1\right) \frac{\frac{2}{3}-\frac{\overline{v^{2}}}{k}}{T}+C_{2} \frac{P}{\rho k}+\frac{\frac{5 \overline{v^{2}}}{T}}{T}+S_{f}
\end{gathered}
$$

The model is a general low-Reynolds number turbulence model that is valid all the way up to solid walls, and therefore does not need to make use of wall functions. However, the $V 2 F$ model has not been well tested and evaluated much in the indoor environment simulations and might encounter numerical stiffness problem in some segregate solution procedures.

\section{j. Reynold Stress Model}

The Reynolds stress model (RSM) is one of the most detailed Reynolds-averaged NavierStokes (RANS) model. The $R S M$ directly solves totally seven transport equations for the Reynolds stresses with an equation for the dissipation rate. Details of each term can be referred in Appendix A

Table A.1.

$$
\frac{\partial}{\partial t}\left(\rho \overline{u_{\imath}^{\prime} u_{j}^{\prime}}\right)+\frac{\partial}{\partial x_{k}}\left(\rho u_{k} \overline{u_{\imath}^{\prime} u_{\jmath}^{\prime}}\right)=D_{T, i j}+D_{L, i j}+P_{i j}+G_{i j}+\phi_{i j}-\varepsilon_{i j}+F_{i j}+S
$$

Although $R S M$ has greater potential to give accurate predictions for complex flows, the accuracy of the RSM is not always superior compared to simpler models and not to mention 
the additional computational expense. Therefore, its applications in indoor air environment simulations remain a careful consideration.

\subsubsection{Results and Discussion}

\section{a. Tile Model Study}

Tile modeling in data center is very important because it reduces the need for detailed modeling of tiny features such as tile pores. Fig. II. 13 shows with and without a $B F$ tile model configuration. With the tile model, the air coming out of the perforated tile is corrected to its actual magnitude. The after-tile velocity, however, failed to match the nearfield measured velocity right after tile. This could be because of insufficient grids to resolve the region near the perforated tile. In fact, it is very hard to deploy such super fine grids at the near-field region after the perforated tile while maintaining a sufficient mesh in other areas. In addition, the four-inch body force region above the tile is only effective outside this region which sees the slow response to the momentum enhancer. Study by Abdelmaksoud et al. [1] also addresses this issue.

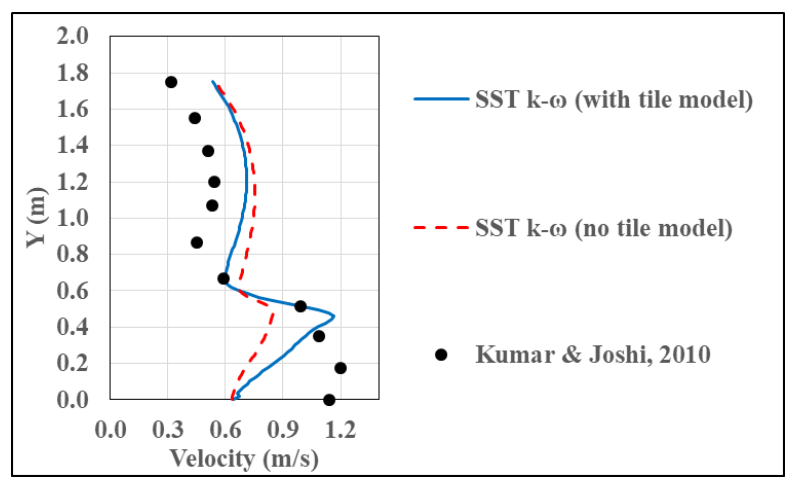

Fig. II.13 Velocity after tile with and without tile model 
Fig. II.14 shows the streamlines of all studied tile models and PIV experiments (EXP) at three different levels of tile-to-server flow rates, i.e., $20 \%$ (low), $60 \%$ (medium), and $100 \%$ (high). In low flow ratio, most of the tile models except the single opening tile model capture the trend of the experimental data. The SO tile model, when using the actual porosity of $31 \%$, shows a dramatic increase in velocity due to a small equivalent tile area specified. Therefore, it completely fails to represent the data in all three scenarios of flow. All tile models start showing clear deviations in medium and high flow ratios.

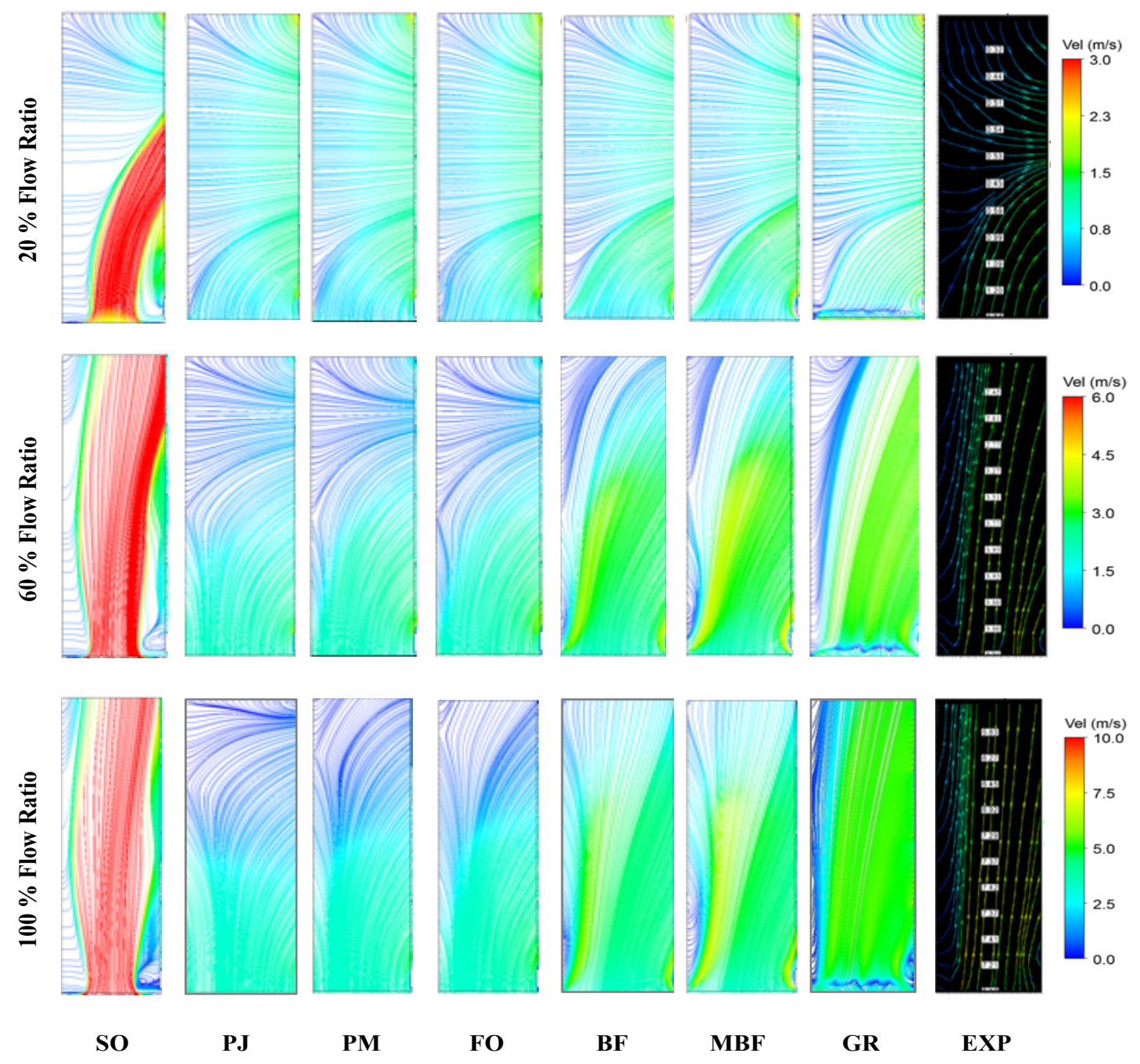

Fig. II.14 Velocity streamlines after tile at three different flow ratios 
On the low end, the $P J$ model, by specifying a pressure jump across the tile surface, poorly predicts the after-tile velocity development, and also fails to represent the far-field velocity resulting in a short rise clearly seen in medium and high airflow ratios. The $P M$ model shows a slight improvement over the $P J$ model due to its three-dimensional nature, yet they are very similar in their features when assuming the air flow is directed perpendicular to the tile surface. The $F O$ model further improved the result, yet still under-predicts it, and again this is expected because of the low perforation tile used.

On the high end, the $B F$ model improves the flow prediction by introducing a momentum source. This enhancement overcomes the momentum deficit in the far-field velocity resulting in a much resolved velocity profile when compared to the experimental data. The $M B F$ tile model further reinforces the flow by adding even a larger magnitude due to its formulation of using vena-contracta velocity instead of the pore velocity. Finally, the most accurate tile model is the geometrical resolution $(G R)$ shown in Fig. II.14; however, it is also the most time- consuming model due to its detailing of all the model features. 

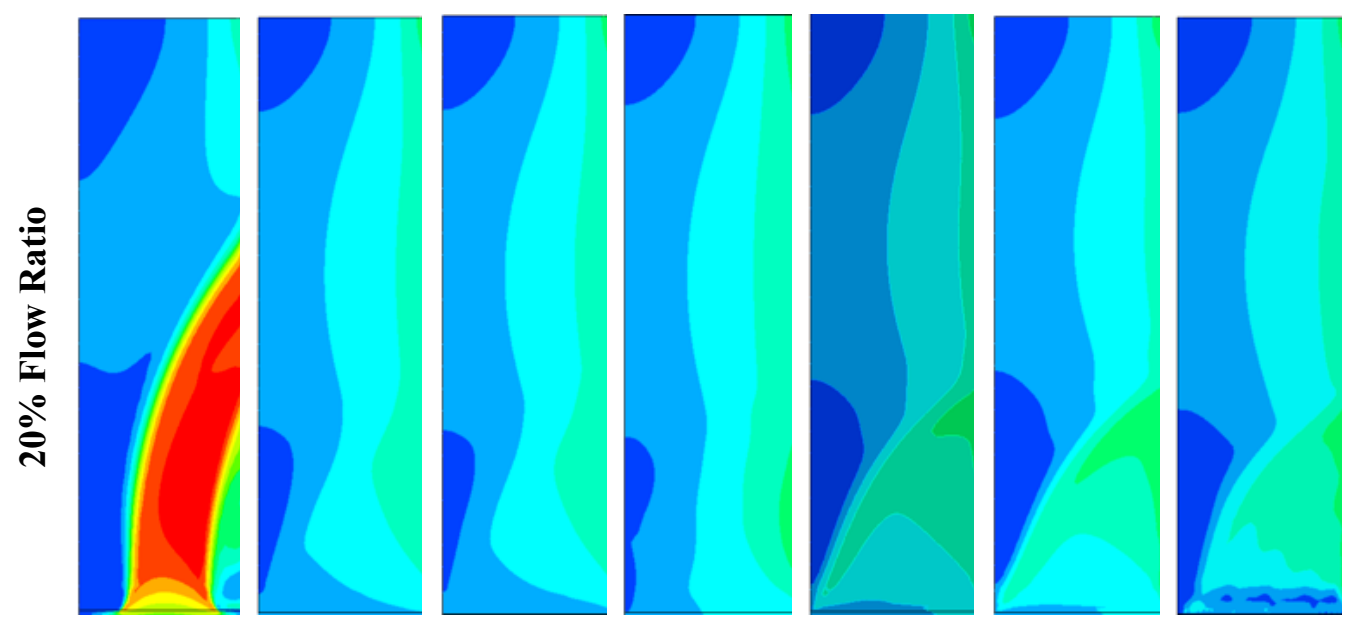

Vel (m/
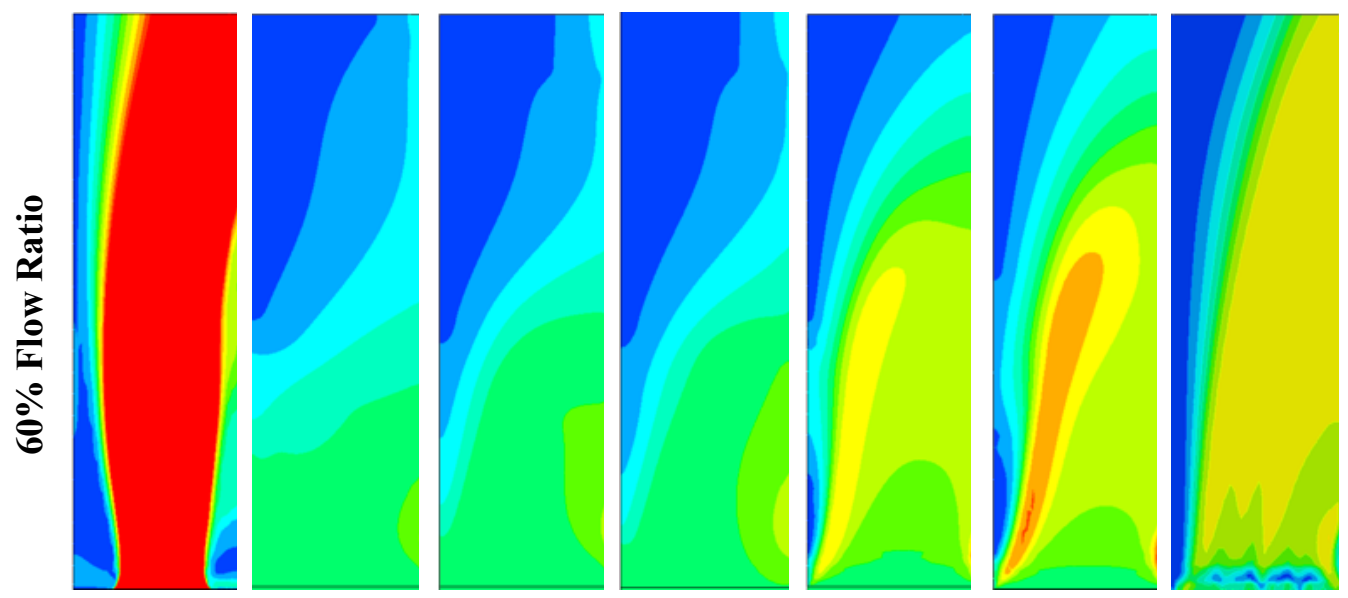

Vel $(\mathrm{m} / \mathrm{s}$

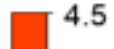

4.0
3.6

- 3.1

2.7

2.3

1.8

1.3

0.9

0.4

0.0
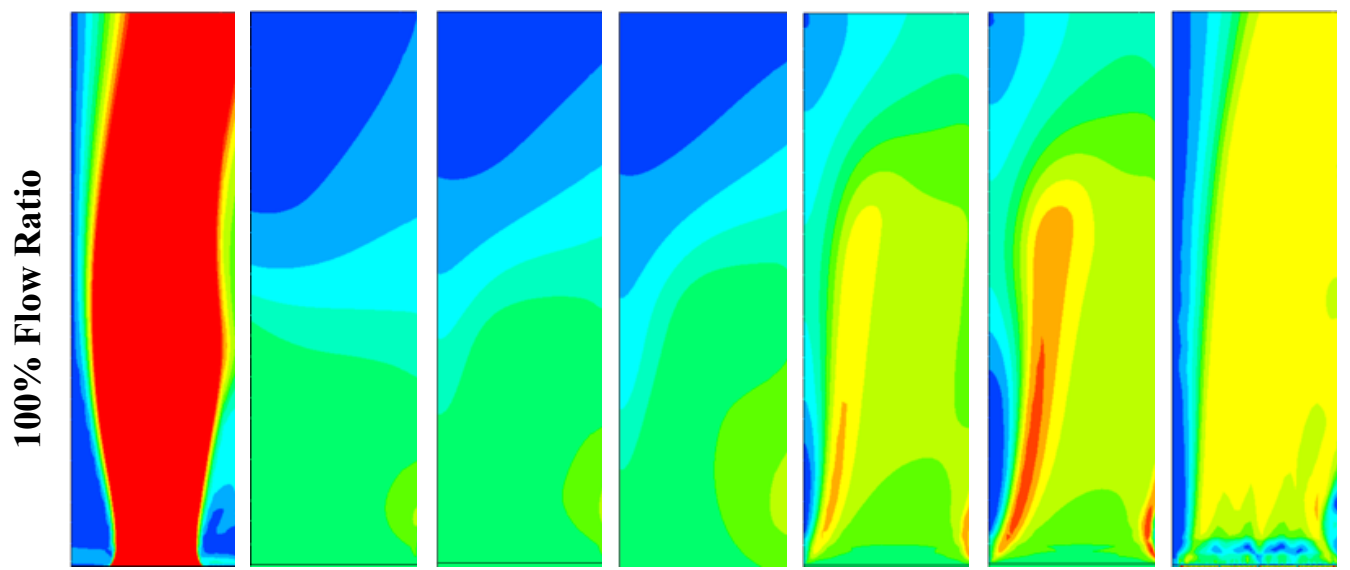

Vel $(\mathrm{m} / \mathrm{s}$

7.0
6.3

5.6

$-4.9$

4.2

3.5

2.8

2.1

1.4

0.7

SO

PJ

PM

FO

BF

MBF

GR

Fig. II.15 Velocity contour for various tile models at three different flow rate ratios 
Fig. II. 15 shows the velocity contours of all tile models. Apart from the single opening tile model, the intensity of the jet velocity is practically improved through simplified tile models, i.e., from $P J$ to $M B F$ models. The $G R$ model contour shows a more realistic resolution of the velocity profile, although there are some deviations of near-field velocity contour after tile due to the insufficient mesh density. As the flow ratio in- creases, the cold jet height also varies in height. The low flow ratio case causes hot air recirculation at top of the server rack, while high flow ratio case allows the cold air plume to reach the entire height of the server rack for sufficient rack cooling.

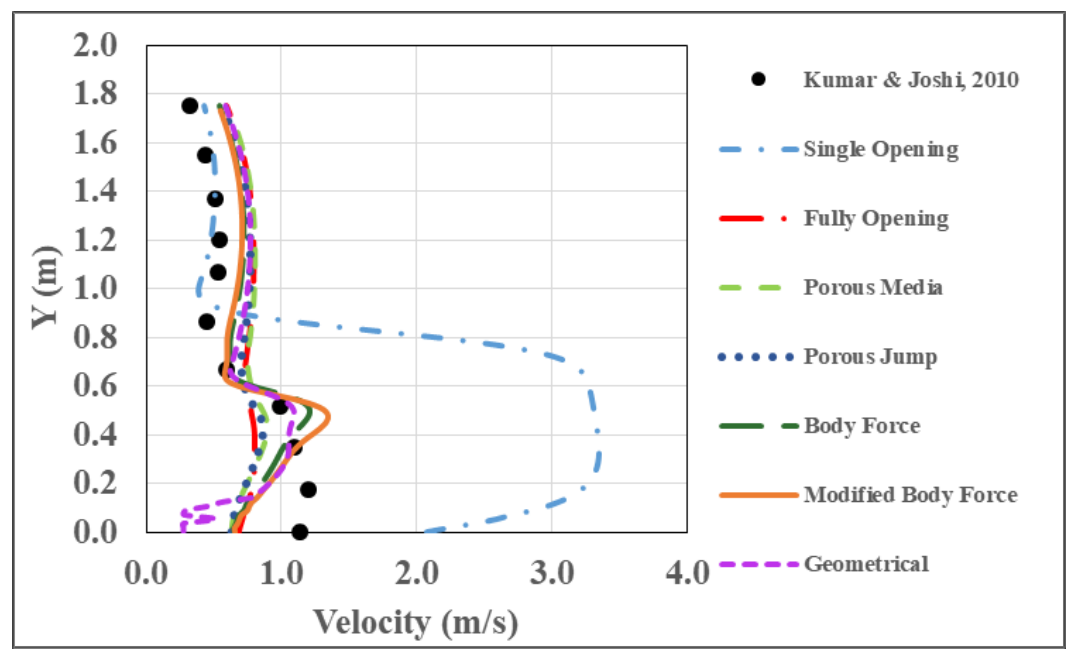

Fig. II. 16 Velocity after tile for different tile models at $20 \%$ flow rate ratio

Fig. II.16 - Fig. II. 18 present the after-tile velocity along the probing line as shown in Fig. II.8 and Fig. II.10. However, instead of directing from the bottom of the flow domain, the lines are plotted from the tile surface all the way to about $1.8 \mathrm{~m}(5.9 \mathrm{ft})$ in height in front of the server rack. In general, the result shows different qualities of tile models, i.e., low and high performance tile models. The results hint that the $P J, P M, F O$, and $S O$ models poorly predict the velocity profile in all three flow ratios, while the $B F$ and $M B F$ models 
agree better. These two models show some differences at a low flow ratio, and distinct changes at medium and high flow ratios with the $M B F$ model showing a better agreement with the data.

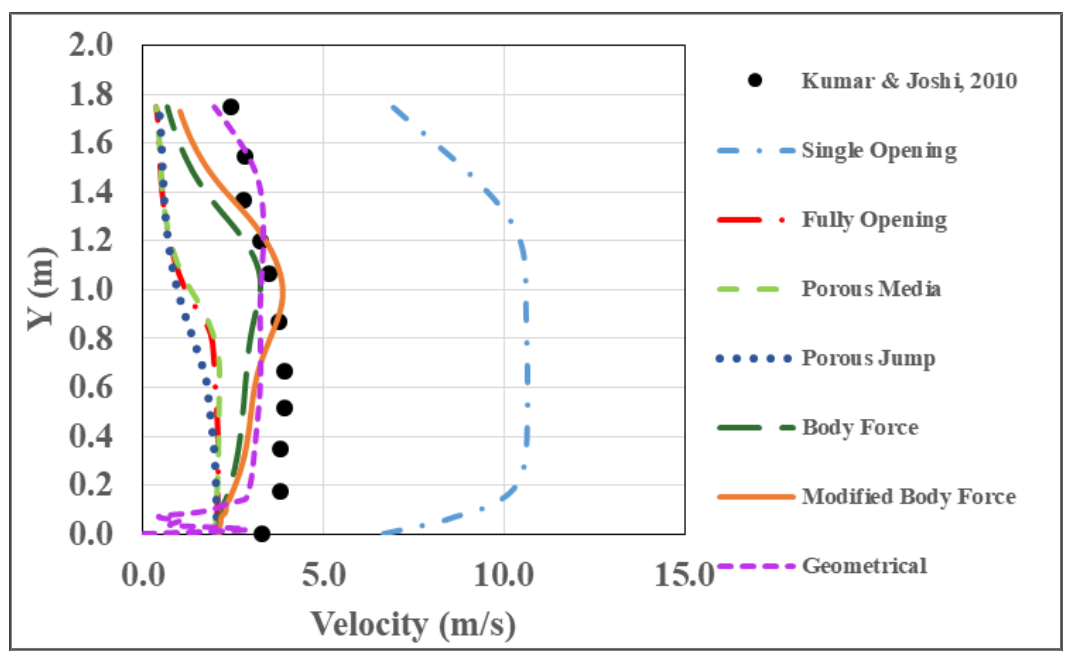

Fig. II.17 Velocity after tile for different tile models at $60 \%$ flow rate ratio

At a low airflow ratio (20\%) as in Fig. II.16, all of the tile models generally capture the trend very well, however, fail to match the upper air level (above $0.61 \mathrm{~m}$ ). There are two possibilities that cause these discrepancies. First, the server rack is situated in a whole data center with adjacent racks and perforated tiles, so there exists an influence of the strong air jet coming from the adjacent tiles. Although the fans in other server racks can be turned off, there is no control on the perforated tiles since they all share the same raised floor plenum. Because of this issue, the experimental data obtained at the upper level might get affected. The difficulty was also reported in [41] where the authors were well aware of this situation. Second, because of the lack of knowing how much of the adjacent air resistance applying on the domain of interest, an appropriate pressure coefficient is not precisely 
known prior to the simulation. Rather, it has been assumed to take the value of $p_{\text {coeff }}=10$ during the simulation process. A thorough characterization of this pressure coefficient is needed during experimental testing to better guide the simulation.

In the medium flow ratio $(60 \%)$ as in Fig. II. 17 , the cold jet experiences less resistance from the hot air recirculation at the top of the server rack. Therefore, it climbs to about $1.0 \mathrm{~m}$ (3.3 ft) and starts to bend a little after losing its initial momentum. While the $S O$ model exaggerates the result, other tile models under-predict the data. $F O, P M$, and $P J$ models fail to match the data, while both $B F$ and $M B F$ models show lower discrepancies compared to the experimental data with the latter shows better agreement. The $G R$ model advances in capturing the trend of the cold jet. However, in the near-field location right above the tile surface, $G R$ model result alters around the starting velocity. This is due to the fact that the poor resolution of the mesh around the small tile pores. A substantial increase in grid size resolves this issue, but it results in a much larger computing time.

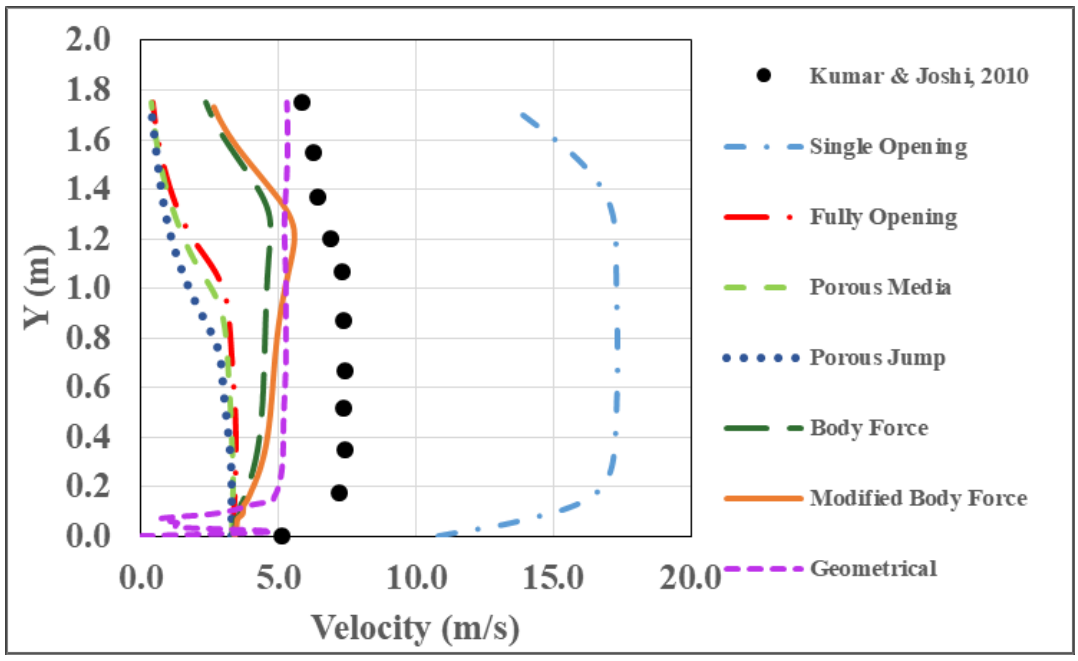

Fig. II. 18 Velocity after tile for different tile models at $100 \%$ flow rate ratio 
In the high flow ratio (100\%) as in Fig. II. 18 where the supply cold air and server flow rates are equal, a strong jet is observed coming out of the tile. All tile models under-predict the magnitude of the air jet velocity, although the $G R$ model has the smallest error. The single opening model, al- though capturing the trend very well, still over predicts the data. Among the simplified tile models, $M B F$ model shows better agreement. As mentioned before, the $G R$ model encounters the same issue at the foot of the air jet due to its insufficient resolution for small tile pores.

\section{b. Turbulence Model Study}

Turbulence models have not been extensively evaluated in data center research. The standard $k$ - $\varepsilon$ turbulence model is widely adopted for data center study [8], [7], [9], [14], [36], [70], [86], [81]. Without justifications and validations, it is very hard to justify whether the standard $k-\varepsilon$ model is the best choice for data center CFD simulation. In the current study, the authors extend the analysis of Reynolds Average Navier-Stokes (RANS) turbulence models to 10 models ranging from zero-equation to seven-equation model. After an evaluation of all available tile models in the previous section, re- commendations are made for more advanced tile models, i.e., $B F$ and $M B F$ models.

First, by utilizing the $B F$ model for tile modeling, we are going to evaluate different turbulence models. Fig. II. 19 shows the velocity profiles at different airflow rate ratios. The results shown in this figure is from the probing line located at the center of the tile 
stretching upward to about $1.75 \mathrm{~m}(5.74 \mathrm{ft})$. At low flow ratio $(20 \%)$, there are not distinct differences among the turbulence models, except for $L R N k$ - $\omega$ and zero-equation models. At medium (60\%) and high (100\%) flow ratios, the turbulence models start to deviate from one another especially at the upper level. Four turbulence models, including $L R N k-\omega, S S T$ $k$ - $\omega$, and realizable $k-\varepsilon$, and standard $k-\varepsilon$ show a better agreement compared to the rest. The zero-equation, though deviating much at high flow ratio, shows the best trend in following the experimental data. In general, all turbulence models under-predict the experimental data.

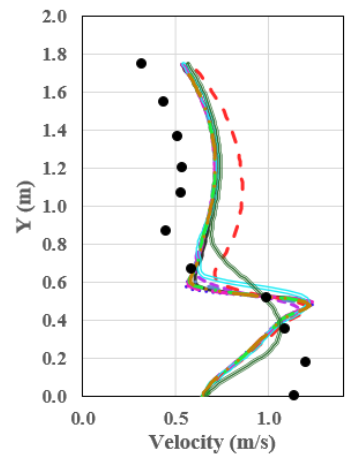

a) $20 \%$

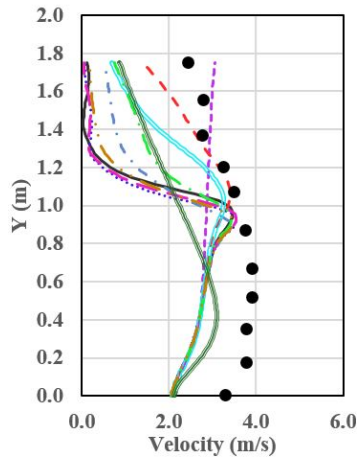

b) $60 \%$

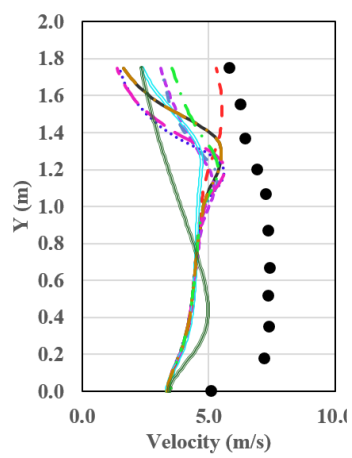

c) $100 \%$

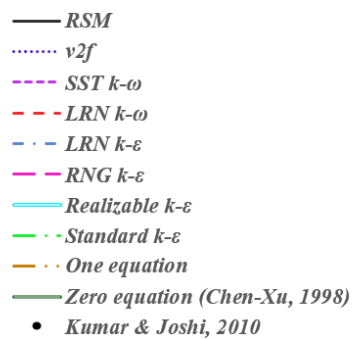

- Kumar \& Joshi, 2010

Fig. II.19 After-tile velocities using BF tile model at different airflow rate ratios

Next, $M B F$ tile model is also tested against different turbulence models. The results are shown in Fig. II.20. Similar to the $B F$ model's results, at low flow ratio (20\%), the velocity difference is minimal except for $L R N \mathrm{k}-\omega$ and zero-equation models. In the medium $(60 \%)$ and high (100\%) flow ratios, the four turbulence models that consistently stand out are also the ones mentioned previously in the $B F$ tile model, i.e., $L R N k-\omega, S S T k$ - $\omega$, realizable $k$ $\varepsilon$, and standard $k-\varepsilon$. These models show a better agreement compared to other turbulence 
models. It is also worthwhile to notice the zero-equation performance which shows an equivalent or better accuracy when compared to the standard $k-\varepsilon$ in all flow ratio scenarios. Again, all turbulence models under-predict the experimental data in all scenarios.

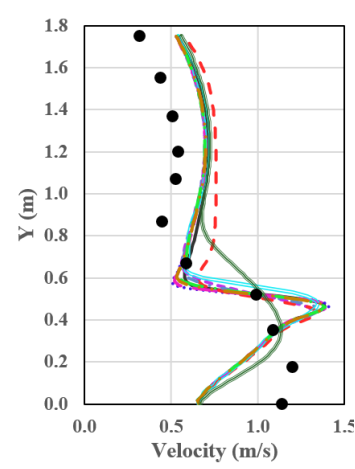

a) $20 \%$

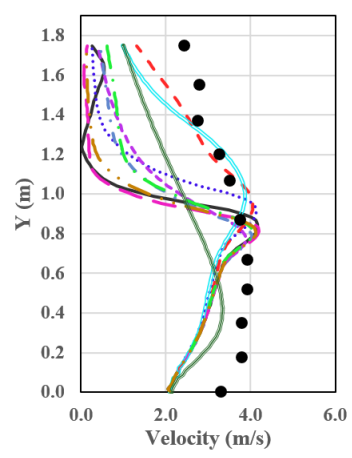

b) $60 \%$

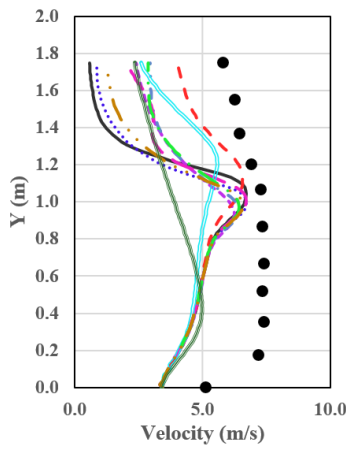

c) $100 \%$

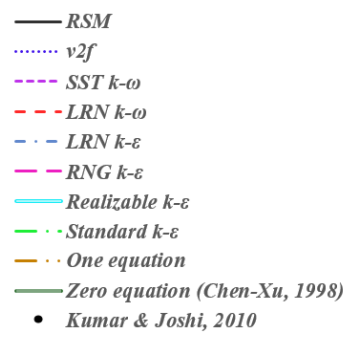

- Kumar \& Joshi, 2010

Fig. II.20 After-tile velocities using MBF tile model at different airflow rate ratios

It is of curiosity to apply the exact measured velocities coming right off the tile surface in all flow ratios. Fig. II.21 shows the results of such applications. In this figure, the velocity profiles generally agree better at the upper level of the tested data than at the bottom level. When an exact tile surface velocity is applied, the turbulence models tends to perform almost the same except for zero-equation model. The airflow resistance uncertainty of the adjacent tiles might account for the mismatch between the simulation result and data, in addition to the challenges from turbulence modeling. If a test is carried out on the quantification of this airflow resistance, it certainly helps to guide the simulation to a more favorable outcome. 


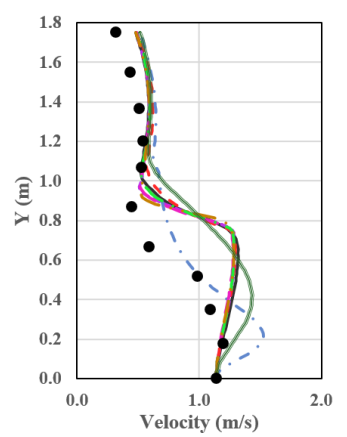

a) $20 \%$

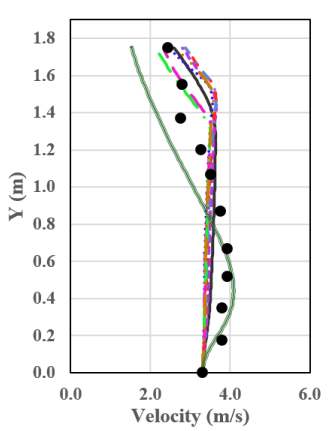

b) $60 \%$

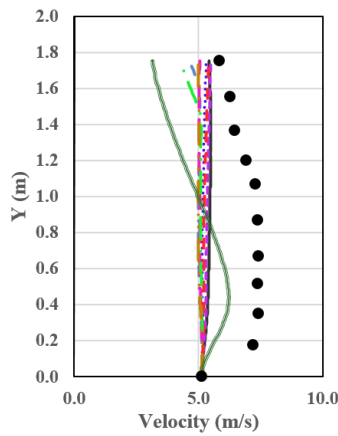

c) $100 \%$

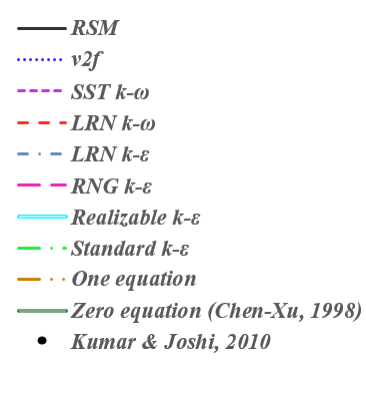

Fig. II.21 After-tile velocities using measured velocity at different airflow rate ratios

The next section carefully quantifies the errors of the studied models for a better insight at these criteria. In addition, recommendations on turbulence models aimed at different purposes are then suggested.

\subsection{Composite Performance Index}

\subsubsection{Error analysis}

This section takes a closer look at the error quantifications and explore the capabilities of the turbulence models through error analysis. Table II.6 shows the quantified root mean square error $(R M S E)$ between the predicted results and experimental data along the probing line as seen in Eq. (II.40).

$$
R M S E=\sqrt{\frac{\sum_{i=1}^{N}\left(\hat{y}_{i}-y_{i}\right)^{2}}{n}}
$$

where $\hat{y}_{i}$ is the simulation values and $y_{i}$ is the experimental data, $n$ represents 11 data points tested in the experimental results. The standard $k-\varepsilon$ model is taken as the baseline case to 
be compared with other turbulence models. Table II.6 shows both the absolute RMSE and the relative error with respect to the baseline standard $k$ - $\varepsilon$ scenario. When the $B F$ tile model is utilized, SST $k-\omega$ and $L R N k-\omega$ have major improvements over the baseline case, while the realizable $k-\varepsilon$ also does well in the medium flow and only worsen off by $3 \%$ and $10 \%$ in the low and high flow scenarios, respectively. One notable point is that at low and medium flow ratios, the zero-equation model also yield a relatively good result compared to the baseline standard $k-\varepsilon$.

Table II.6 Error assessment for different turbulence models at three flow ratios

\begin{tabular}{|c|c|c|c|c|c|c|c|c|c|c|}
\hline $\begin{array}{c}\text { Air Flow } \\
\text { Ratio }\end{array}$ & $\begin{array}{c}\text { Standard } \\
k-\varepsilon\end{array}$ & $\begin{array}{c}R N G \\
k-\varepsilon\end{array}$ & $\begin{array}{c}\text { Realizable } \\
k-\varepsilon\end{array}$ & $\underset{k-\varepsilon}{L R N}$ & $\begin{array}{c}L R N \\
k-\omega\end{array}$ & $\begin{array}{l}S S T \\
k-\omega\end{array}$ & $V 2 F$ & $R S M$ & $S-A$ & $\begin{array}{l}\text { Zero } \\
\text { Eqn }\end{array}$ \\
\hline \multicolumn{11}{|c|}{ Root mean square error (BF model) } \\
\hline$f_{1}$ & 0.239 & 0.241 & 0.247 & 0.324 & 0.299 & 0.237 & 0.249 & 0.241 & 0.238 & 0.245 \\
\hline$f_{2}$ & 1.286 & 1.834 & 1.150 & 1.524 & 0.935 & 0.926 & 1.857 & 1.794 & 1.737 & 1.265 \\
\hline$f_{3}$ & 2.540 & 2.988 & 2.806 & 2.659 & 2.388 & 2.473 & 3.006 & 2.808 & 2.883 & 3.065 \\
\hline \multicolumn{11}{|c|}{ Relative error with respect to the baseline standard $k-\varepsilon$ (BF model) } \\
\hline$f_{1}$ & $0 \%$ & $1 \%$ & $3 \%$ & $36 \%$ & $25 \%$ & $-1 \%$ & $4 \%$ & $1 \%$ & $0 \%$ & $3 \%$ \\
\hline$f_{2}$ & $0 \%$ & $43 \%$ & $-11 \%$ & $19 \%$ & $-27 \%$ & $-28 \%$ & $44 \%$ & $40 \%$ & $35 \%$ & $-2 \%$ \\
\hline$f_{3}$ & $0 \%$ & $18 \%$ & $10 \%$ & $5 \%$ & $-6 \%$ & $-3 \%$ & $18 \%$ & $11 \%$ & $14 \%$ & $21 \%$ \\
\hline \multicolumn{11}{|c|}{ Root mean square error (MBF model) } \\
\hline$f_{1}$ & 0.222 & 0.222 & 0.240 & 0.221 & 0.258 & 0.220 & 0.222 & 0.230 & 0.222 & 0.237 \\
\hline$f_{2}$ & 1.599 & 1.972 & 0.920 & 1.574 & 0.808 & 1.484 & 1.961 & 1.915 & 1.873 & 1.123 \\
\hline$f_{3}$ & 2.525 & 2.707 & 2.456 & 2.556 & 2.033 & 2.654 & 3.330 & 3.372 & 3.129 & 2.825 \\
\hline \multicolumn{11}{|c|}{ Relative error with respect to the baseline standard $k-\varepsilon$ (MBF model) } \\
\hline$f_{1}$ & $0 \%$ & $0 \%$ & $8 \%$ & $0 \%$ & $16 \%$ & $-1 \%$ & $0 \%$ & $4 \%$ & $0 \%$ & $7 \%$ \\
\hline$f_{2}$ & $0 \%$ & $23 \%$ & $-42 \%$ & $-2 \%$ & $-49 \%$ & $-7 \%$ & $23 \%$ & $20 \%$ & $17 \%$ & $-30 \%$ \\
\hline$f_{3}$ & $0 \%$ & $7 \%$ & $-3 \%$ & $1 \%$ & $-19 \%$ & $5 \%$ & $32 \%$ & $34 \%$ & $24 \%$ & $12 \%$ \\
\hline
\end{tabular}


When $M B F$ tile model is used, four outstanding turbulence models include realizable $k$ - $\varepsilon$, $L R N k-\varepsilon, L R N k-\omega$, and $S S T k-\omega$. The $L R N k-\omega$ shows $50 \%, 19 \%$ improvement in the medium and high flow ratios, respectively, although it is worsen by $16 \%$ at the low flow condition. The realizable $k-\varepsilon$ shows improvement of $42 \%$ and $3 \%$ in the medium and high flow relative to the standard $k-\varepsilon$, while it is worse off by $8 \%$ in the low flow scenario. The other two models, i.e., $L R N k-\varepsilon$ and $S S T k$ - $\omega$, either improve or worsen by a marginal figure. Finally, it is very interesting that the simplest zero-equation model also does very well compared to the baseline model, standard $k-\varepsilon$.

Table II.7 Error assessment for different tile models at three flow ratios

\begin{tabular}{|c|c|c|c|c|c|c|c|}
\hline Air Flow Ratio & SO & $\boldsymbol{P J}$ & $\boldsymbol{P M}$ & $\boldsymbol{F O}$ & $\boldsymbol{B F}$ & MBF & GR \\
\hline \multicolumn{7}{|c|}{ Root mean square error } \\
\hline$f_{\boldsymbol{1}}$ & 1.474 & 0.297 & 0.284 & 0.28 & 0.247 & 0.24 & 0.223 \\
\hline $\boldsymbol{f}_{2}$ & 6.551 & 2.168 & 2.077 & 2.124 & 1.15 & 0.92 & 0.546 \\
\hline $\boldsymbol{f}_{3}$ & 9.667 & 4.883 & 4.816 & 4.621 & 2.806 & 2.456 & 1.852 \\
\hline \multicolumn{7}{|c|}{ Relative error with respect to the baseline Single Opening (SO) } \\
\hline $\boldsymbol{f}_{\boldsymbol{1}}$ & $0 \%$ & $-80 \%$ & $-81 \%$ & $-81 \%$ & $-83 \%$ & $-84 \%$ & $-85 \%$ \\
\hline $\boldsymbol{f}_{2}$ & $0 \%$ & $-67 \%$ & $-68 \%$ & $-68 \%$ & $-82 \%$ & $-86 \%$ & $-92 \%$ \\
\hline$f_{3}$ & $0 \%$ & $-49 \%$ & $-50 \%$ & $-52 \%$ & $-71 \%$ & $-75 \%$ & $-81 \%$ \\
\hline
\end{tabular}

On different tile models evaluation, similar assessment using the standard turbulence model is also made of the errors at the probing line for each tile model (see Table II.7). As expected, the level of accuracy increases as the further development of more advanced models, such as $B F$ and $M B F$ tile models, are considered. The single opening, due to the use of exact porosity, results in a worst scenario in all studied flow rate ratios. The $P M$ model slightly improves the result, while the $F O$ model remains in a close tie with it. In the upper-level tile models, $B F$ and $M B F$ show better accuracy. The $G R$ model delivers the 
least error (shown as largest negative numbers in percentage in the table) compared to other models.

\subsubsection{Computing Time Evaluation}

The computing time is also considered in evaluating the turbulence models used in this study. Since the total computational depends on many factors such as a fine-tuned underrelaxation factor, a better metric for comparison maybe the time per iteration. In order to have a more consistent determination of this, a calculated time per iteration from hundred iterations is considered. As expected, the simulation time increases as the number of equations solved in each turbulence model increases. The least time required is for the zeroequation with only $2.546 \mathrm{~s}$, while it is $5.071 \mathrm{~s}$ for the seven-equation $R S M$ model. The steady increase in average time per iteration is plotted in Fig. II.22a.

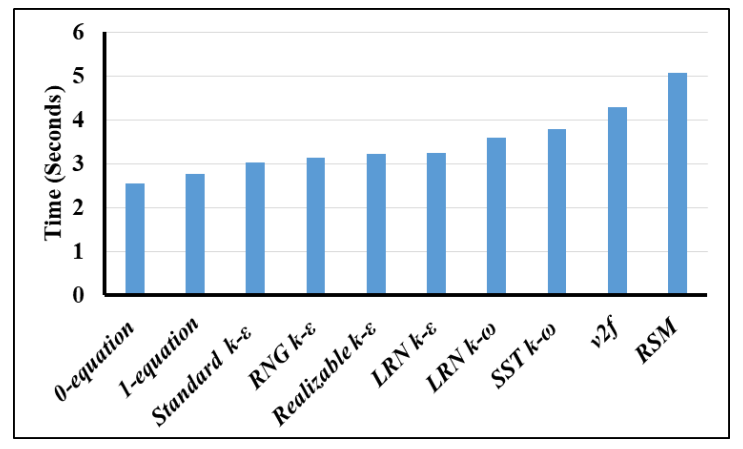

a)

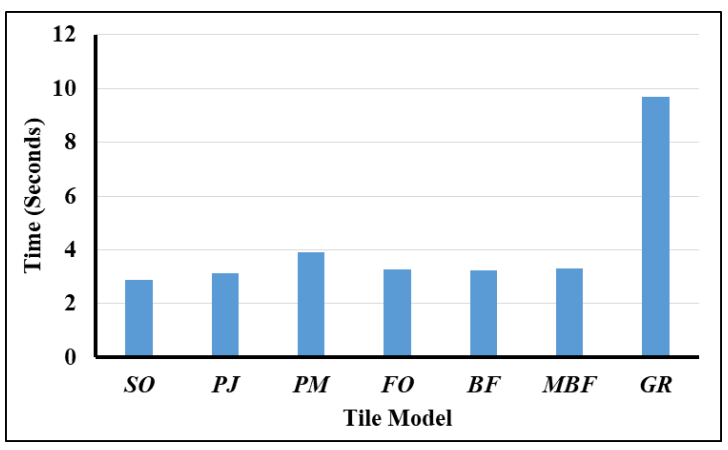

b)

Fig. II.22 Average time per iteration for different (a) turbulence models, (b) tile models

As for tile model, Fig. II.22b shows that the geometrical resolution model is the most timeconsuming model, and single opening yields least time with $9.701 \mathrm{~s}$ and $2.865 \mathrm{~s}$, respectively. $B F$ and $M B F$ models are very close, with the latter slightly higher. 


\subsubsection{Composite Performance Index}

The simulation results discussed in terms of time and accuracy for different tile and turbulence models are carefully examined in the previous sections. It is necessary to look for a recommendation within the scope of the study. Baseline cases for tile and turbulence model assessment are identified as single opening, and standard $k-\varepsilon$, respectively. In terms of time assessment, a non-dimensional time ratio of the average time per iteration divided by the baseline value is calculated as seen in Eq. (II.41). As for the accuracy, a collective average non-dimensional number is defined in Eq. (II.42) to evaluate the overall accuracy performance for each turbulence model. The idea is to calculate the average relative error over the baseline case of all the flow ratio scenarios.

$$
\begin{gathered}
t_{r, m}^{*}=\frac{t_{m}}{t_{b}} \\
a_{r, m}^{*}=\left[\frac{\sum_{f=1}^{n}\left(\frac{a_{f}}{a_{f, b}}\right)}{n}\right]_{m}
\end{gathered}
$$

$\boldsymbol{a}_{\boldsymbol{r}, \boldsymbol{m}}^{*}=$ dimensionless relative average errors, $\boldsymbol{t}_{\boldsymbol{r}, \boldsymbol{m}}^{*}=$ dimensionless relative average time, $m$ $=$ turbulence model, $n=$ number of cases studied, $f=$ flow ratio, $b=$ baseline

The calculated results for Eqs. (II.41) and (3.32 are presented in Table II.8. In this table, essentially the baseline standard $k-\varepsilon$ and single opening both receive an index of 1.0 for both $t_{r}$ and $a_{r}$, and all other turbulence and tile models have either a value greater or less than one. For both metrics, values greater than one signify a worse case compared to the baseline, and less than one suggests an improvement over the baseline case. 
Table II.8 Non-dimensional index in terms of time and accuracy

\begin{tabular}{|c|c|c|c|c|c|c|c|c|c|c|}
\hline \multicolumn{11}{|c|}{ For turbulence assessment } \\
\hline & $\begin{array}{c}\text { Standard } \\
k-\varepsilon\end{array}$ & $\begin{array}{c}R N G \\
k-\varepsilon\end{array}$ & $\underset{k-\varepsilon}{R e a l i z a b l e}$ & $\begin{array}{c}L R N \\
k-\varepsilon\end{array}$ & $\begin{array}{c}L R N \\
k-\omega\end{array}$ & $\begin{array}{l}S S T \\
k-\omega\end{array}$ & $V 2 F$ & $R S M$ & $S-\boldsymbol{A}$ & Zero Eqn \\
\hline $\boldsymbol{t}_{r}^{*}$ & 1.000 & 1.033 & 1.065 & 1.073 & 1.183 & 1.248 & 1.414 & 1.671 & 0.914 & 0.839 \\
\hline$a_{r}^{*}$ & 1.000 & 1.153 & 0.944 & 1.097 & 0.898 & 0.943 & 1.202 & 1.180 & 1.149 & 1.017 \\
\hline \multicolumn{11}{|c|}{ For tile assessment } \\
\hline & SO & $P J$ & \multicolumn{2}{|c|}{$P M$} & FO & & $B F$ & \multicolumn{2}{|c|}{$M B F$} & $G R$ \\
\hline$t_{r}^{*}$ & 1.000 & 1.094 & \multicolumn{2}{|c|}{1.362} & 1.142 & & 1.128 & \multicolumn{2}{|c|}{1.159} & 3.386 \\
\hline$a_{r}^{*}$ & 1.000 & 0.346 & \multicolumn{2}{|c|}{0.336} & 0.331 & & 0.211 & \multicolumn{2}{|c|}{0.186} & 0.142 \\
\hline
\end{tabular}

Depending on the purpose of the user, one might need a quick simulation for an estimation or prediction, while the other focuses on the accuracy of the simulation results. Three scenarios are created in Table II.9, i.e., the accuracy-targeting models (ATMS), the timetargeting models (TTMs), and well-balanced models $(W B M s)$. The time $(W \mathrm{t})$ and accuracy $(\mathrm{Wa}$ ) weighing factors are specified depending upon the goal of each scenario. For example, the $W t$ and $W a$ both receive a value of 0.5 in the well-balanced model scenario.

Table II.9 Weighing factors for different scenarios

\begin{tabular}{|c|c|c|}
\hline Scenarios & Time weighing factor & Accuracy weighing factor \\
& $\left(W_{t}\right)$ & 0.9 \\
\hline Accuracy-targeting models (ATMs) & 0.1 & 0.1 \\
\hline Time-targeting models (TTMs) & 0.9 & 0.5 \\
\hline Well-balanced models (WBMs) & 0.5 & \\
\hline
\end{tabular}


Finally, the overall performance index $(P I)$ for each turbulence and tile model based on the respective scenarios are evaluated as in Table II.10 based on the formulation specified in Eq. (II.43). Technically, a lower PI indicates a more desired model. As for turbulence model assessment in regard to the $A T M s$, three turbulence models that yield a better performance than the baseline standard $k-\varepsilon$ are identified as realizable $k-\varepsilon, L R N k-\omega$, and SST $k-\omega$. These models show an index less than one after an overall performance is assessed. Meanwhile, the zero-equation model also yields an equivalent index compared to the baseline, which signifies the attention for this much simpler model. For the TTMs, two favorable models are identified as the zero-equation and the one-equation models. While the zero-equation has a performance index less than one, the one-equation model has an index close to the baseline standard $k-\varepsilon$. Finally, in the WBMs, only the zeroequation stands out as a candidate to advance over the baseline with an index of 0.928 . All other models are either worse or equivalent to the baseline case.

$$
P I=W_{t} t_{r}^{*}+W_{a} a_{r}^{*}
$$

As for tile model assessment, for all two out of three objectives, i.e., $A T M$ and $W B M$, it is clear that $B F$ and $M B F$ models are doing better than any other tile models with lowest desired PI. In the TTM objective, there are no appreciable time differences among the tile models, except for $P M$ and $G R$ models. The $G R$ model is generally not desired due to its highly time-consuming characteristic and is also reflected through the PI in Table II.10. The rest of tile models are not as good as the $B F$ and $M B F$ models with higher PIs. Therefore, it makes sense to use these two best tile models for the turbulence model study in the earlier section of this study. 
Finally, it is necessary to identify a good combination of tile and turbulence model for current work. After a comprehensive assessment of both tile and turbulence models, it is recommended that for time-targeting and well-balance models assessment, the zeroequation turbulence model, coupled with either a body force or modified body tile model yields a good outcome. When considering accuracy-targeting model, the zero-equation coupled with $B F$ or $M B F$ models yields an equivalent performance compared to standard $k-\varepsilon$.

Table II.10 Overall performance index

\begin{tabular}{|c|c|c|c|c|c|c|c|c|c|c|}
\hline \multicolumn{11}{|c|}{ Turbulence model assessment } \\
\hline & $\begin{array}{c}\text { Standard } \\
k-\varepsilon\end{array}$ & $\begin{array}{r}R N G \\
k-\varepsilon\end{array}$ & $\begin{array}{c}\text { Realizable } \\
\qquad-\varepsilon\end{array}$ & $\begin{array}{l}L R N \\
k-\varepsilon\end{array}$ & $\begin{array}{l}L R N \\
k-\omega\end{array}$ & $\begin{array}{l}S S T \\
k-\omega\end{array}$ & $V 2 F$ & $R S M$ & $S-A$ & $\begin{array}{l}\text { Zero } \\
\text { Eqn }\end{array}$ \\
\hline ATM & 1.000 & 1.141 & 0.956 & 1.095 & 0.927 & 0.974 & 1.223 & 1.229 & 1.126 & 0.999 \\
\hline TTM & 1.000 & 1.045 & 1.053 & 1.075 & 1.155 & 1.218 & 1.393 & 1.622 & 0.938 & 0.857 \\
\hline$W B M$ & 1.000 & 1.093 & 1.005 & 1.085 & 1.041 & 1.096 & 1.308 & 1.426 & 1.032 & 0.928 \\
\hline \multicolumn{11}{|c|}{ Tile model assessment } \\
\hline & SO & & $P J$ & $P M$ & $F O$ & & $B F$ & $M B F$ & & $G R$ \\
\hline$A T M$ & 1.000 & & 0.421 & 0.439 & 0.412 & & 0.303 & 0.283 & & 0.466 \\
\hline$T T M$ & 1.000 & & 1.019 & 1.259 & 1.061 & & 1.036 & 1.062 & & 3.062 \\
\hline$W B M$ & 1.000 & & 0.720 & 0.849 & 0.736 & & 0.670 & 0.672 & & 1.764 \\
\hline
\end{tabular}

\subsection{Remarks}

In the first part of the chapter, a coupled viscous and non-viscous domain solution method has been used to couple a zero-equation model and the standard $k$ - $\varepsilon$ model for application in a data center model. A detailed procedure and criteria on how to separate the whole 
domain into sub-domains are discussed. A complete numerical solution on different turbulence models was carried out, and the choice of zero-equation model over Euler equations in the non-viscous domain has certain benefits on the current data center model. In the coupled numerical solution, the viscous domain was simulated with the standard $k$ $\varepsilon$ turbulence model, while zero-equation was utilized in the non-viscous domain. Within the investigated parameter ranges, the result of the coupling method is reasonably close to the baseline case, while consumes only half of the simulation time used in the baseline case. However, the computational time reduction by the hybrid turbulence model method might be geometry-specific or application-specific, which needs further investigation to confirm.

In the second part of the chapter, a comprehensive study of tile models is carried out and validated against the available experimental data. Among the tile models studied, two advanced tile models, the body force and modified body force models, show good agreement with the tested data, while keeping the simulation time reasonable by avoiding the exhaustive detail of tile modeling. Ten turbulence models in the RANS family are then carefully evaluated based on the two suggested good tile models. In addition, three levels of flow ratios between the tile air supply and the server air flow are also considered to investigate the diverse turbulence models within a reasonably wide range.

By emphasizing on the time and accuracy of each turbulence model, a detailed assessment based on the proposed overall composite performance index of tile and turbulence models is carried out to identify the suitable models for different application purposes. Three types 
of proposed models, i.e., accuracy-targeting, time-targeting, and well-balanced models are out- lined based on proper weighing factors. Although for the accuracy-targeting models, realizable $k-\varepsilon, L R N k-\omega$, and $S S T k-\omega$ turbulence models stand out as preferable ones, the zero-equation model is also worth noticing with an equivalent performance with the baseline standard $k$ - $\varepsilon$. Also the zero-equation model shows better results compared to standard $k-\varepsilon$ model when time-targeting and well-balanced models are considered.

In a similar way, when assessing different tile models, both accuracy and time are the main concerns. It is concluded that the body force and modified body force models yield good accuracy while consuming almost the same amount of computational time compared to other tile models, except for the geometrical resolution and porous media models. Finally, the best combination recommended for the current study to yield the most favorable outcome is the use of zero-equation turbulence model with either body force or modified body force tile model. 
CHAPTER III REDUCED ORDER MODELING 


\subsection{Proper Orthogonal Decomposition (POD) Method for Data Center Study}

In this chapter, thermal modeling of data center is carried out using a POD method based on CFD data. Compared to existing works, the scope includes: (1) A model data center with different configurations is used for the computational study. This model data center $\left(\mathrm{L} \times \mathrm{W} \times \mathrm{H}=45.9^{\prime} \times 32.8^{\prime} \times 11.5^{\prime}\right)$ is a well-representative of a real data center, which has three hot aisles and two cold aisles, and four CRAH units placed at four opposite walls. It is different from the study of Samadiani and Joshi [58], [59], [60] where only two CRAH units are placed at the end of the rack rows against the walls. (2) The effects of the number of parameters used for the POD basis function on the accuracy of the thermal modeling of data centers are investigated. The key differences between the current study and previous works also lie upon the number of input parameter used to construct the POD basis function. In the current study, we apply the POD method to obtain the temperature profile of a new representative data center model using three parameters, i.e., the rack heat load, mass flow rate, and an additional parameter which is the inlet temperature. (3) An interpolation technique of constructing 3-D picture of the temperature profiles using 2-D POD simulation is introduced for data center modeling at different conditions. Most of the previous studies that use POD method on data centers focus on 2-D temperature and/or velocity distribution POD representation with the variation of server load and velocity at the inlet for their studied cases. The 2D-based modeling method for a 3D data center model construction is carried out to study the effects of multiple design parameters including inlet air mass flow rate $(5.5 \mathrm{~kg} / \mathrm{s}-7.3 \mathrm{~kg} / \mathrm{s})$, rack heat load $(9 \mathrm{~kW}-18 \mathrm{~kW}$ per rack), and also a new parameter which is also important in data center thermal management, i.e., rack inlet 
air temperature $\left(12.0{ }^{\circ} \mathrm{C}-13.2{ }^{\circ} \mathrm{C}\right)$. Case studies are generated from these parameter combinations. Sensitivity analysis of the effects of each design parameter on the temperature is done to assess the fidelity of the POD model as compared to the CFD model.

\subsubsection{Methodologies}

\section{a. Fundamentals of Proper Orthogonal Decomposition}

The Proper Orthogonal Decomposition (POD), depending on different fields and applications, is also called the discrete Karhunen-Loève transform in signal processing, the Hotelling transform in multivariate quality control, singular value decomposition of $\boldsymbol{X}$, eigenvalue decomposition of $\boldsymbol{X}^{T} \boldsymbol{X}$ in linear algebra, just to name a few. This is a reduced order modeling technique that finds applications in computationally processing large amount of high-dimensional data with the aim of obtaining low-dimensioning descriptions that capture much of the phenomena of interest. It is a powerful tool that can be used to numerically predict the temperature and flow fields much faster as compared to conventional full-field CFD/HT modeling.

In the application of thermal/fluid system, the method of snapshots introduced by Sirovich [65] is usually used to reduce the calculation effort. The fundamental idea of using this

method is to decompose a set of temperature profile $T^{(k)}=T_{i, j}^{(k)}$ or velocities distribution $V^{(k)}=(u, v)_{i, j}^{(k)}$, where $i, j$ is the index of the grid points in the temperature or velocities 
distributions, $k$ is the snapshot index, into a linear combination of $M$ spatial basis functions (POD modes, $\left.\phi_{m}\right)$ and the corresponding coefficients $a_{m}^{(k)}$

$$
T^{(k)}=\sum_{m=1}^{M} a_{m}^{(k)} \phi_{m}
$$

One restriction to this method is that the basis functions must be orthonormal to each other. The goal of using POD technique is to find a sequence of orthonormal basis functions $\phi_{m}$ (POD modes) representing the 'coherent structures' such that the error of the orthogonal projection is minimized

$$
\sum_{k=1}^{K}\left\|T^{(k)}-\sum_{m=1}^{M} a_{m}^{(k)} \phi_{m}\right\|^{2} \rightarrow \text { minimized }
$$

subject to $\left(\phi_{i}, \phi_{j}\right)=\delta_{i j}=\left\{\begin{array}{l}1 \text { if } i=j \\ 0 \text { if } i \neq j\end{array}\right.$

where $\|\cdot\|$ denotes the $\mathrm{L}^{2}$ norm.

This minimization effort leads to the eigenvalue problem, where the functions $\phi$ are the eigenfunctions. The idea of the snapshot POD is not to solve the eigenvalue problem to obtain the functions $\phi$, but to write the basis function, $\phi$, depending on the given snapshots:

$$
\phi=\sum_{k=1}^{M} a^{(k)} T^{(k)}
$$

The eigenvalue problem is described as,

$$
C A=\lambda A
$$

where $A=\left(a_{1}, a_{2}, \ldots, a_{M}\right)^{T}$ are the eigenvectors corresponding to the eigenvalues $\lambda=$ $\left(\lambda_{1}, \lambda_{2}, \ldots, \lambda_{M}\right), C$ is called the correlation matrix (which is an $m \times m$-matrix) with elements $c_{i j}=\frac{1}{M}\left(q_{i}, q_{j}\right)$, and $(\because)$ denotes an inner product. Thus, the problem is further 
reduced to an $m$ dimensional eigenvalue problem. While an approximation to any desired accuracy in eq. (III.1) can be always be obtained if $M$ can be chosen large enough, we may like to choose a cutoff value $P<M$ to further represent approximately the total "energy" contained in the $m$ POD modes. By keeping only the first $P$ eigenfunctions, the temperature field can be expressed as

$$
T^{(k)} \approx \sum_{p=1}^{P} a_{p}^{(k)} \phi_{p}
$$

One way to increase the accuracy of the approximation is the representation of the temperature field in the summation of the mean average temperature $\left(T_{m}\right)$ and their fluctuating values calculated from the POD method.

$$
T^{(k)}=T_{m}+\sum_{1}^{p} a_{p}^{(k)} \phi_{p}
$$

The POD energy obtained from the first $P$ modes is defined as

$$
E=\sum_{i=1}^{P} \lambda_{i}
$$

where $\lambda_{i}$ is the eigenvalue. The energy fraction, $e$, from the first $P$ modes is, thus, defined as the ratio of the first $P$ modes over the entire $M$ modes. If one wants the error in the POD basis to be less than some prescribed tolerance $\delta$, then the value of $P$ is chosen to be the smallest integer such that the total energy fraction from the first $P$ modes is greater than $(1-\delta)$

$$
e=\frac{\sum_{i=1}^{P} \lambda_{i}}{\sum_{i=1}^{M} \lambda_{i}} \geq 1-\delta
$$

In order to apply the POD based method to certain high-order models with different design parameters, the Galerkin projection is applied to project the state-space model onto the 
POD modes $\left(\left\{\varphi_{n}\right\}\right)$ which is previously calculated. The computation of such process is summarized in the following algorithm.

Step 1: Generate the observations using CFD/HT simulation.

Step 2: Solve the eigenvalue problem eq. (III.4) to get the eigenvalues and their corresponding eigenfunctions (or POD basis functions).

Step 3: Calculate the POD coefficients by using Galerkin projection.

Step 4: Generate the POD temperature/velocities fields as in eq. (III.5).

\section{b. Three-Dimensional Approach}

For three-dimensional representation of the temperature field, we propose that the aforementioned POD process is applied on selected planes. The observational snapshots were first extracted from CFD simulation to construct the POD basis for each of these planes, and then the case study was run to obtain the meaningful results. The compilation of several planes at certain critical locations allows to retrieve missing information. That is, one can interpolate temperature as well as velocity information sandwiched between two simulated planes. The formulation for mid-plane interpolation, assuming 2-D POD surfaces are constructed in the XZ plane, can be referred to eq. (III.9). This method significantly reduces the computational effort in reconstructing the 3-D temperature and velocity distributions of the data center model.

$$
T_{i, j}^{(k)}=\left(T_{i, j}^{(k)}\right)_{n}+\frac{\left(y-y_{n}\right)+\left[\left(T_{i, j}^{(k)}\right)_{n+1}-\left(T_{i, j}^{(k)}\right)_{n}\right]}{y_{n+1}-y_{n}}
$$

where $n=\frac{\Delta y}{d}+1$

The aggregate number of planes for 3-D visualization is thus calculated as 
$n($ original $)+n-1($ interpolated $)=2 n-1$.

However, if the distance between two selected planes is large, the temperature difference between $\mathrm{CFD} / \mathrm{HT}$ and POD results will be significant. In contrast, if the distance is too small then more selected planes need to be simulated for the 3D construction of the data center model.

In the real case scenario, each of these selected surfaces could become probing plane where temperature sensors or flow meters are placed. The data points obtained from such planes are further analyzed using POD method to find a sequence of orthonormal basis functions (POD modes) representing the 'coherent structures.' Probing planes can be designed such that sensors are placed on movable carts that aligned on the same plane. The carts will simultaneously move from one side of the computer room to the other side to complete the 3-D picture of the temperature field. Only certain planes are selected to visualize the temperature field in the data center room. In some other scenarios, one can use only a line of sensors and sweep rough a certain direction to complete one plane of information at a time, then move on to the next plane and keep scanning the domain plane by plane. This method tremendously reduces the number of utilized sensors since only a line of sensors is used. However, it is less accurate as compared to a plane of sensors scanning the entire domain. The order of data acquisition in the line of sensors method can be summarized in these following steps.

Step 1: Obtain an $N-S$ line in the $x-y$ plane

Step 2: Move to the next line in the direction of $E-W$ to complete the plane of information 
Step 3: Move to the next plane in the $B-T$ direction or $z$ direction to complete scanning the entire domain

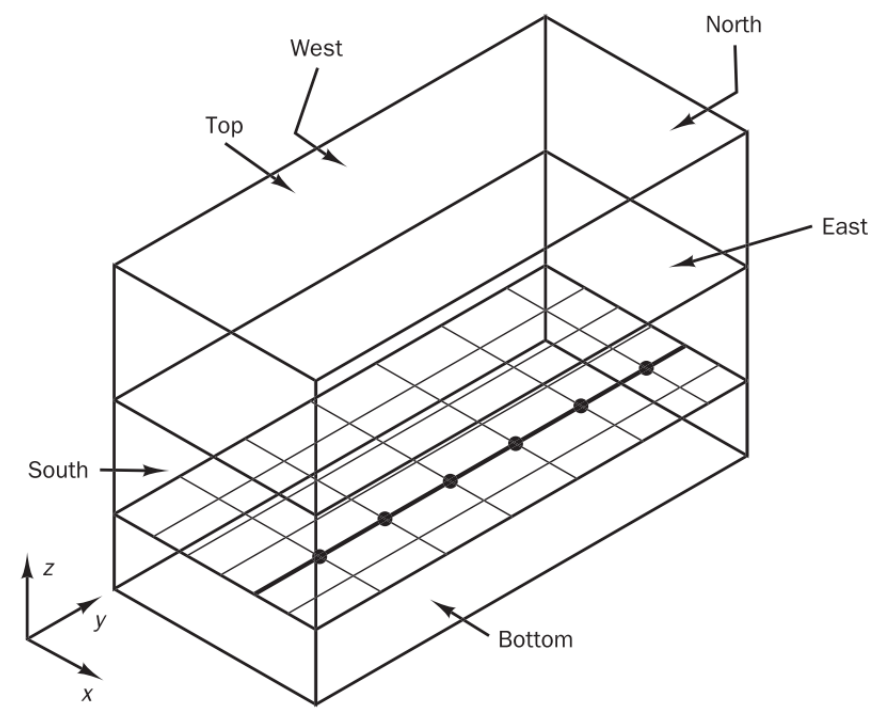

Fig. III.1 Application of sweeping method a three-dimensional geometry

\subsubsection{Data Center Modeling}

\section{a. Data Center Description}

In this study, the data center model was adopted from the one that has been mentioned in Chapter 3 (See Fig. II.1). Each server releases heat ranging from $0.9 \mathrm{~kW}-3 \mathrm{~kW}$ depending on different cases. There are four CRAC units placed in the middle of four different walls with a cooling capacity of 100 tons per CRAC. Each CRAC provides about 9,137 CFM 12,626 CFM to the pressurized 2-foot raised floor plenum. Each server receives from 32.5 to $45.1 \mathrm{CFM}$ of cool air from the fully open perforated floor tiles. The data center was configured using the current best practice of hot and cold aisles (Fig. III.2). The server racks 
are placed in such a way that their back sides are facing each other to form hot aisles, while the front sides, which receive cooling air from the perforated floor tiles, form cold aisles.

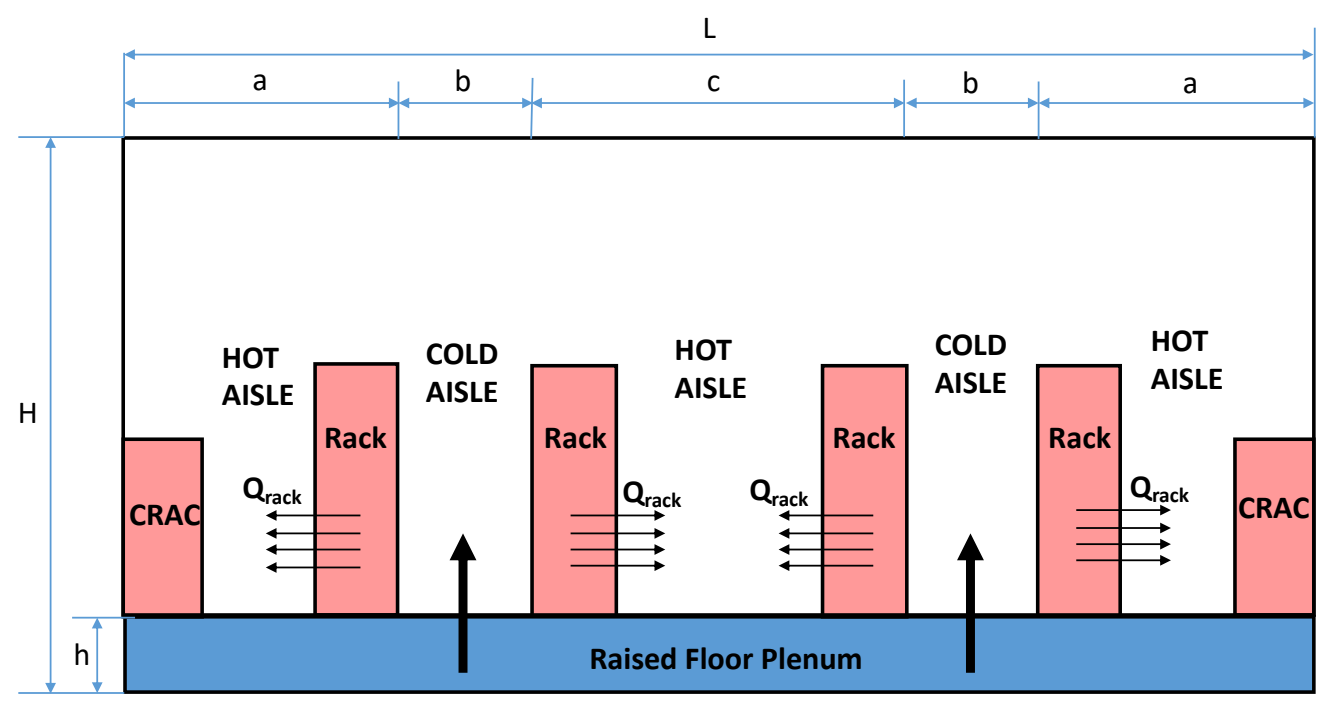

Fig. III. 2 Hot and cold aisle practice in the current model

As for the purpose of constructing POD based reduced order model of temperature field, the CRAC unit is modeled as a constant mass flow inlet and exhaust fan exit, discharging the cooling air into the raised floor plenum at temperature varied from $11.8^{\circ} \mathrm{C}$ to $15.8^{\circ} \mathrm{C}$. The return hot air to the CRAC is assumed to be constant of $27^{\circ} \mathrm{C}$ with a target constant mass flow of $6 \mathrm{~kg} / \mathrm{s}$. Also, the rack heat loads and CRAC air mass flow rate are specified to change between $900 \mathrm{~W}$ and $3000 \mathrm{~W}$, and $5.5 \mathrm{~kg} / \mathrm{s}$.

\section{b. CFD/HT Modeling}

Initially, the mesh independence study was carried out with an ultimate 615,696 grid cells led to a very small further change in maximum temperature. Therefore, the mesh with 
616,696 grid cells is considered fine enough and used for the numerical simulation study. Reynold average Navier-Stokes (RANS) model was considered for the current CFD simulation. The continuity, momentum, and energy equations are presented in eq. (III.10) - (III.13) with the assumption of incompressible, constant-property flow, and negligible radiation.

$$
\begin{aligned}
& \frac{\partial}{\partial x_{i}}\left(\bar{u}_{i}\right)=0 \\
& \rho \frac{\partial}{\partial t}\left(\bar{u}_{i}\right)+\rho \frac{\partial}{\partial x_{j}}\left(\bar{u}_{i} \bar{u}_{j}\right)=-\frac{\partial \bar{P}}{\partial x_{i}}+\mu \frac{\partial^{2} \bar{u}_{i}}{\partial x_{j} x_{j}}+\frac{\partial \tau_{i j}}{\partial x_{j}} \\
& \text { where, } \tau_{i j}=-\rho_{o} \overline{u_{\imath}^{\prime} u_{j}^{\prime}}=\left|\begin{array}{lll}
\overline{u^{\prime 2}} & \overline{u^{\prime} v^{\prime}} & \overline{u^{\prime} w^{\prime}} \\
\overline{u^{\prime} w^{\prime}} & \overline{v^{\prime 2} w^{\prime}} & \overline{v^{\prime} w^{\prime}} \\
\overline{w^{\prime 2}}
\end{array}\right| \\
& \frac{\partial \bar{T}}{\partial t}+\frac{\partial \bar{u}_{\imath} \bar{T}}{\partial x_{i}}=\alpha \frac{\partial^{2} \bar{T}}{\partial x_{i} x_{i}}+\frac{\partial \overline{u_{l}^{\prime} T^{\prime}}}{\partial x_{i}} \\
& \text { Where, } \alpha=\frac{k}{\rho C_{p}} \text {, and } \overline{u_{l}^{\prime} T^{\prime}}=-\alpha_{t} \frac{\partial \bar{T}}{\partial x_{i}} \text { and } \alpha_{t}=\frac{v_{t}}{\sigma_{t}}\left(0.7<\sigma_{t}<0.9\right) \\
& \frac{\partial \bar{T}}{\partial t}+\frac{\partial \bar{u}_{\iota} \bar{T}}{\partial x_{i}}=\alpha \frac{\partial^{2} \bar{T}}{\partial x_{i} x_{i}}-\alpha_{t} \frac{\partial^{2} \bar{T}}{\partial x_{i} x_{i}}
\end{aligned}
$$

The instantaneous temperature (T) consists of the average temperature $(\bar{T})$ and turbulent temperature $\left(\overline{T^{\prime}}\right)$ terms follows the constitutive law for the heat flux vector (Fourier's law). The thermal diffusivity $(\alpha)$ is calculated as a constant value, while the turbulent thermal diffusivity $\left(\alpha_{t}\right)$ is calculated based on the turbulent kinematic viscosity $\left(v_{t}\right)$ and turbulent Prandtl number $\left(\sigma_{t}\right)$. Next, we will choose a turbulent model for the calculations of these two parameters. For standard $k-\varepsilon$ turbulence model, $\sigma_{t}$ is a user-specified constant values between 0.7 and 0.9 . The turbulent kinematic viscosity $\left(v_{t}\right)$ is calculated based off eq. (16). When selecting the turbulence mode for this study, the standard $k-\varepsilon$ turbulence model, as 
shown in eq. (III.14) - (III.16), was selected for CFD modeling simulation to obtain observational cases as a basis for POD, as well as the case study for 15 different temperature fields. Its reasonable accuracy has been well-tested in [3], [8], [7], [9], [14], [36], [70], [86]. Because of its robustness and economy, the standard $k$ - $\varepsilon$ turbulence model is popular for flows that have complex geometries and heat transfer as compared to other turbulence models. In general, data centers normally have relatively small pressure gradient and this particularly best suits the current turbulence model. The following turbulence model constants were used $C \mu=0.09, C_{1 \varepsilon}=0.09, C_{2 \varepsilon}=1.92, \operatorname{TKE} \operatorname{Pr}=1.0$.

$$
\begin{gathered}
\frac{\partial}{\partial t}(\rho k)+\frac{\partial}{\partial x_{i}}\left(\rho k \bar{u}_{i}\right)=\frac{\partial}{\partial x_{j}}\left[\left(\mu+\frac{\mu_{t}}{\sigma_{k}}\right) \frac{\partial k}{\partial x_{j}}\right]+G_{k}+G_{b}-\rho \varepsilon \\
\frac{\partial}{\partial t}(\rho \varepsilon)+\frac{\partial}{\partial x_{i}}\left(\rho \varepsilon \bar{u}_{i}\right)=\frac{\partial}{\partial x_{j}}\left[\left(\mu+\frac{\mu_{t}}{\sigma_{\varepsilon}}\right) \frac{\partial \varepsilon}{\partial x_{j}}\right]+C_{1 \varepsilon} \frac{\varepsilon}{k}\left(G_{k}+C_{3 \varepsilon} G_{b}\right)-C_{2 \varepsilon} \rho \frac{\varepsilon^{2}}{k} \\
\mu_{t}=\rho C_{\mu} \frac{k^{2}}{\varepsilon}
\end{gathered}
$$

where $G_{k}=-\rho \overline{u_{\imath}^{\prime} u_{\jmath}^{\prime}} \frac{\partial \bar{u}_{j}}{\partial x_{i}}$ representing the production of turbulence kinetic energy.

$G_{b}=\beta g_{i} \frac{\mu_{t}}{P r_{t}} \frac{\partial \bar{T}}{\partial x_{i}}$ representing the buoyancy turbulence generation term.

$C_{1 \varepsilon}, C_{2 \varepsilon}$, and $C_{3 \varepsilon}$ are constants

$\sigma_{k}$, and $\sigma_{\varepsilon}$ are the turbulence Prandtl numbers.

The model was simulated with the standard wall function. The phase coupled SIMPLE algorithm was used for the velocity-pressure coupling. Least squares cell based was used for gradient in spatial discretization. Momentum, volume fraction, turbulent kinetic energy, and turbulent dissipation rate equations were solved by using first order upwind scheme. 


\section{c. POD Modeling}

The procedure for running POD reduced order modeling is carried out by the procedure at the end of section 4.1.1a. Following the completion of CFD simulation, POD reduced order modeling is then constructed. First, the observations/snapshots are extracted from the CFD full simulation results by the combination of either two or three different parameters that influence the characteristics of air flow and temperature field. In Table 3.1, two parameters including the inlet air mass flow rate and the server rack heat dissipation are varied to form the POD basis for the case studies. In order to compare the accuracy of the POD basis functions, we constructed a second table that consists one more parameter to consider, i.e., inlet air temperature. Table 3.2 shows 20 observations laid out with the combinations of three design parameters. These observations serve as a POD basis for the case studies where each parameter is investigated separately to understand their influences.

Next, the parametric study is carried out. For case $1-5$, rack inlet temperature ranges from $12.0^{\circ} \mathrm{C}$ to $13.2^{\circ} \mathrm{C}$, while air mass flow rate and rack heat dissipation are kept constant. For case $6-10$, air mass flow rate is changed from $6.5 \mathrm{~kg} / \mathrm{s}$ to $7.3 \mathrm{~kg} / \mathrm{s}$, while other parameters are held constant. For case $11-15$, the rack heat dissipation is changed from $900 \mathrm{~W}$ to $1800 \mathrm{~W}$, and the other parameters are unchanged. Table 3.3 shows the details of such arrangement. The effect of each parameter is discussed in the next section. 


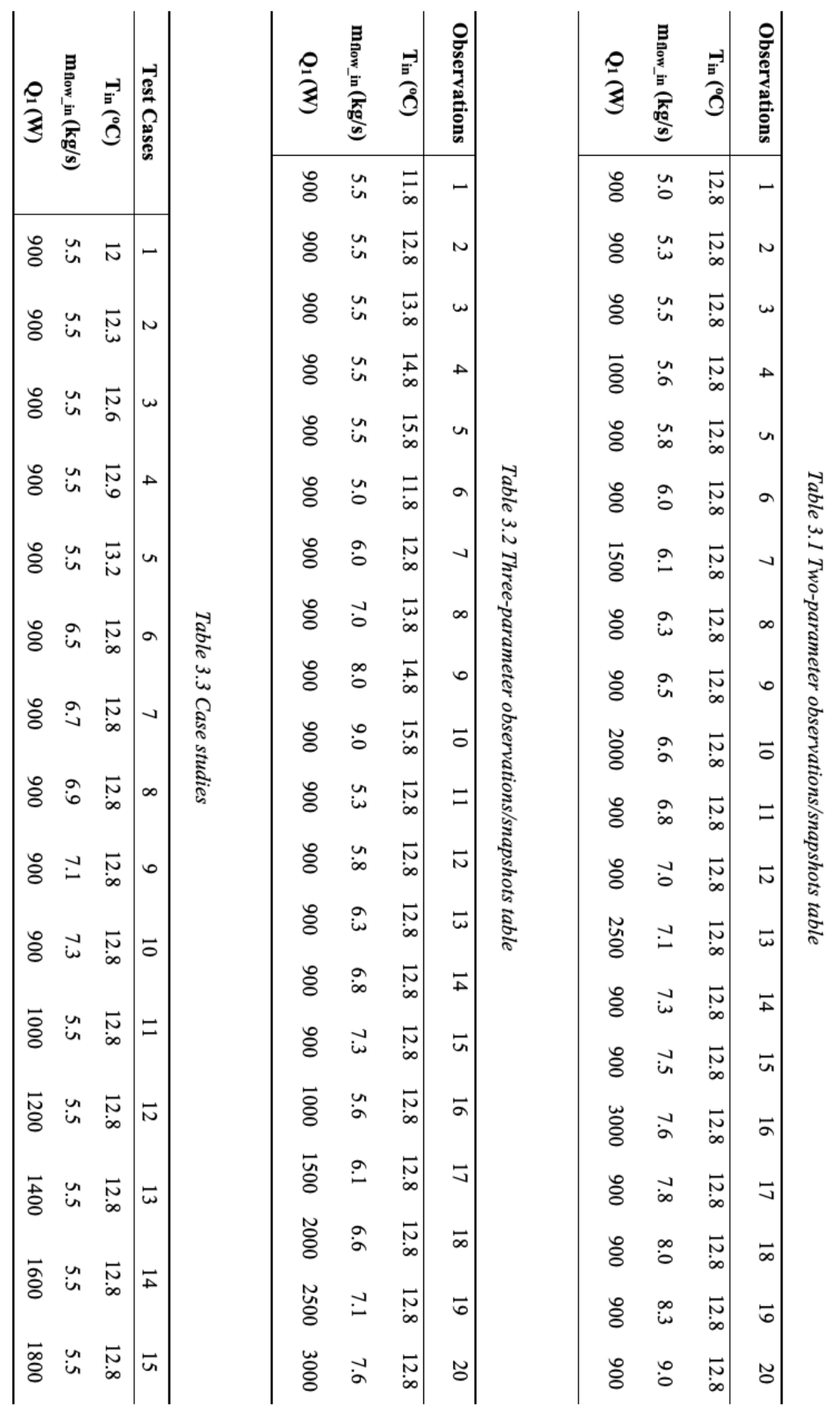


The energy captured by each POD mode in the system is proportional to the relevant eigenvalue. The cumulative energy percentage captured by each POD mode is plotted against the mode number in Fig. III.3. It is seen that the first six modes capture $99.9 \%$ of the energy and the principal eigenfunction contributes $98 \%$ of the total. This reveals that with just a few POD modes the coherent structure can be established.

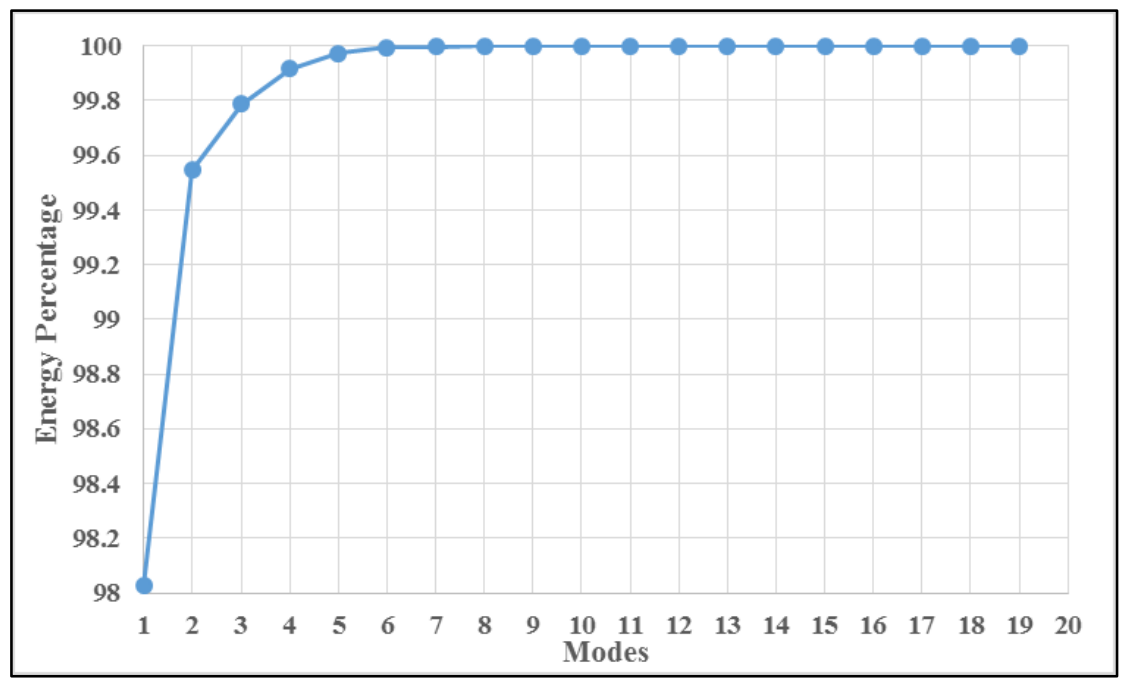

Fig. III.3 Cumulative energy percentage captured in each POD model

One of the advantages of using POD reduced order modeling is the dominant coefficients that capture most of the energetic features of the temperature field. By looking at the plot of POD coefficients versus modes, one can see the behavior of the POD coefficients during the change in POD modes. Indeed, fifteen cases as seen in Fig. III.4 have shown dramatic decrease after about first 10 modes. Apparently, it is shown that a larger data set can be reconstructed coherently with only a few modes. This is advantageous in boosting the processing time when it comes to real time sensor data. Indeed, the POD method is effectively used to gather sensor data and gives quick insights of the current situation in 
the data center by cutting off unnecessary trailing modes without concerning of losing much accuracy.

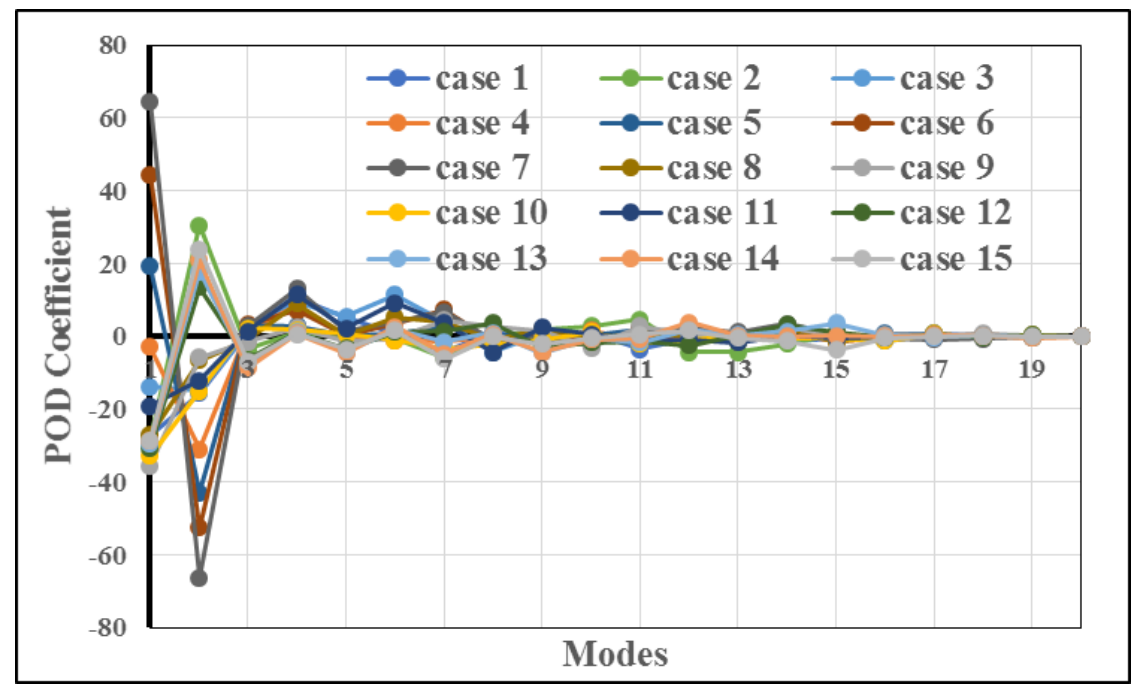

Fig. III.4 POD coefficients of different modes for four different test cases

The POD method is compared with the full CFD solutions for the accuracy assessment.

Fig. III.5 shows the mean temperature difference of fifteen cases. The mean temperature difference is defined as the average of the absolute values of the temperature difference between POD and full numerical predictions for all the points. The temperature difference converges after about first 8 modes for almost all cases. The mean temperature errors rapidly stabilize within the first three modes. Cases $6-10$ show a more rapid decrease with higher mean temperature errors. Recall that cases $6-10$ are associated with the change in inlet mass flow rate. This reveals the solution related to the inlet mass flow rate condition tends to stabilize faster and has a more profound effect on the mean error as compared to the other two conditions. 


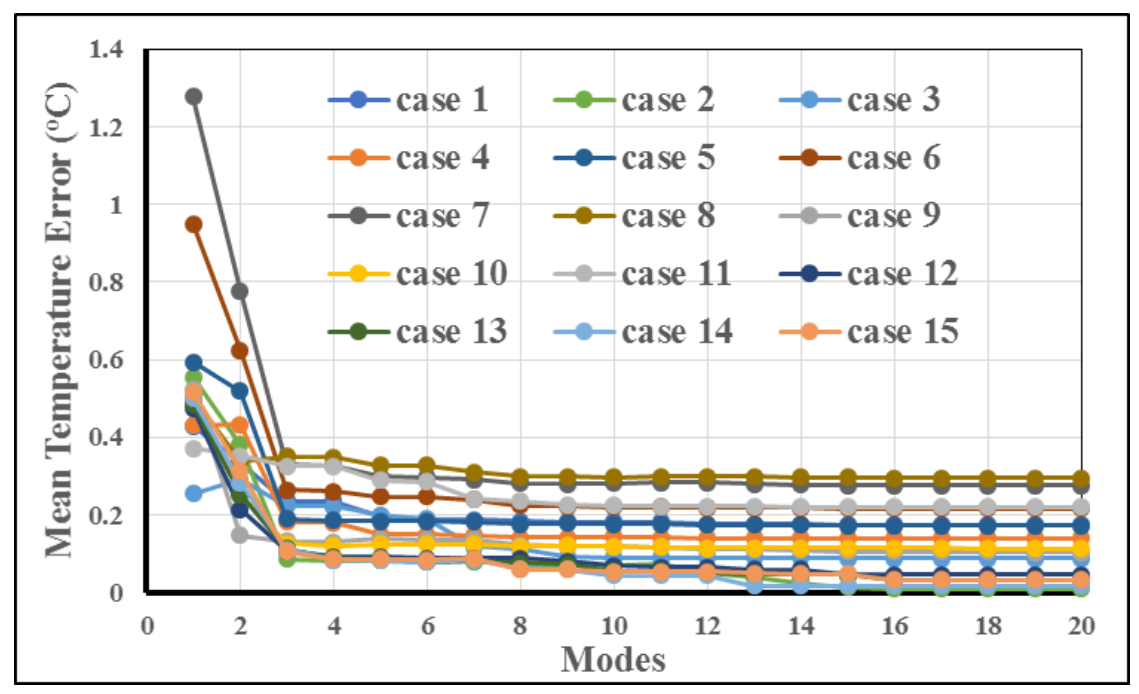

Fig. III.5 Mean temperature vs. modes

The effect of rack inlet temperature (cases $1-5$ ), inlet mass flow rate (cases $6-10$ ), and server heat load (cases $11-15$ ) are respectively observed in Fig. III.6. The mean temperature differences between CFD and POD for all the cases shown from the middle plane $(\mathrm{Y}=5 \mathrm{~m})$ also distinguish the difference between two- and three-parameter observations. The offset in temperature difference between these two observations scenarios goes up to $1.75^{\circ} \mathrm{C}$ at some cases. In particular, the rack inlet temperature tends to produce no change in temperature difference for the two-parameter scenario, while it is slightly increased for the three-parameter scenario. Both inlet mass flow rate and server heat load conditions generate a similar trend for both scenarios. As for the mass flow inlet condition, it is interesting to observe the temperature difference surges at a lower mass flow rates and settles flat at higher flows. The temperature difference is a steady increase in the case of server heat load condition. Overall, the three-parameter observation gives a smaller temperature difference in all the cases as compared to the two-parameter observation. This is an interesting finding regarding the effect of the number of 
observational parameters involved in the construction of the POD basis functions. The second conclusion seen from Fig. III.6 is that there are almost no changes or minimal changes in the temperature difference in the case of rack inlet temperature variation, whereas it is a steady increase in the case of server heat load rises. The inlet mass flow rate condition, in contrast, peaks at lower and settles at higher flow rates.

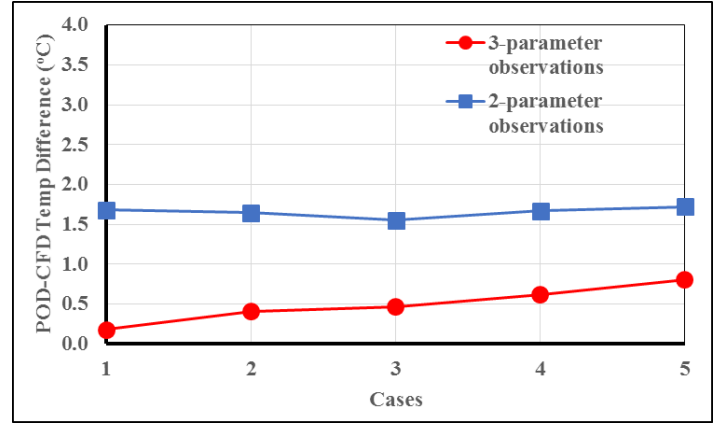

a) Rack inlet temperature,

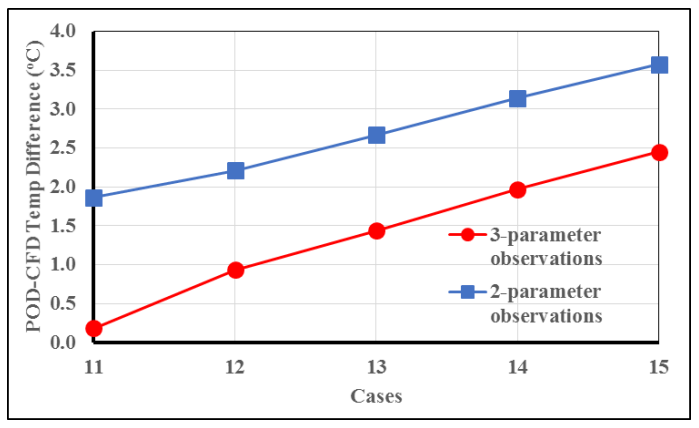

c) Server heat load

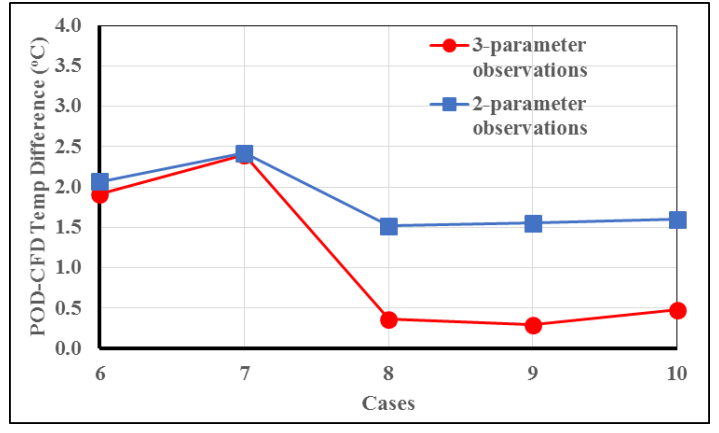

b) Mass flow rate

Fig. III.6 Two- and three-parameter observations comparison at various design inputs

From Fig. III.6, one can see that the temperature difference between POD and CFD magnifies when the design condition supplied to the data center is insufficient which results in a higher air temperature profile in the data center. In fact, in the effect of mass flow rate, keeping server heat load and rack inlet air temperature constant and decreasing the mass flow rate, the discrepancy between POD and CFD tends to be higher as seen in the cases 6 and 7. Once the mass flow rate is sufficiently provided, the temperature difference drops 
below $1.0^{\circ} \mathrm{C}$. The same observation can be concluded in the effect of server heat load and rack inlet air temperature. As each of these parameters increases while keeping other parameters constant, it results in a higher temperature difference between CFD and POD because of the fact that the original temperature profile is very high. As long as we keep the design condition within a reasonable zone, the discrepancy between CFD and POD stays low. This observation can be seen in these cases 1,2,3 (effect of rack inlet air temperature), 11, 12 (effect of server heat load), 8, 9, 10 (effect of mass flow rate) from Fig. III.6.

Next, we will see how temperature difference varies along the $y$ direction in Fig. III.7. The temperature difference is typically high in the region near the end of the server rack rows $(\mathrm{Y}=1 \mathrm{~m}$ to $\mathrm{Y}=3 \mathrm{~m}$ ), which is normally vulnerable to hot spots due to insufficient cooling. Since the air temperature surrounding the server racks has a direct effect to the servers, a high quality of the result is required. Therefore, the lower temperature difference in these areas means a more accurate temperature profile obtained from the POD method.
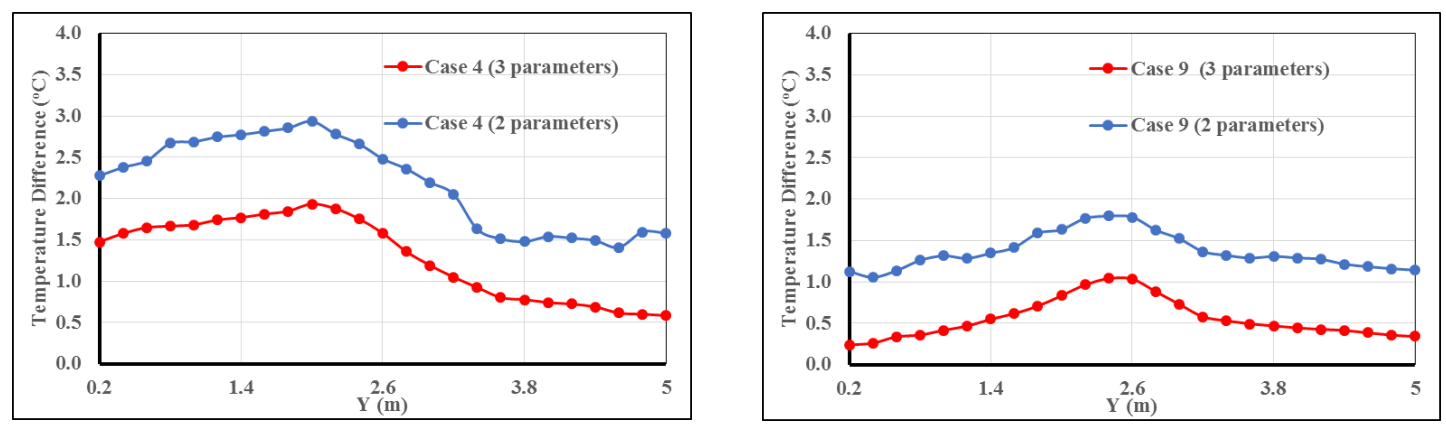

Fig. III.7 Average temperature difference between POD-CFD in the XZ plane 
Fig. III.8 shows the temperature profile among 2-observation POD, 3-observation POD, and CFD results for three representative cases $(4,5$, and 15$)$. It is clear that the 3observation results in both cases show a closer match with CFD results, which confirms the discussion in Fig. III.7.
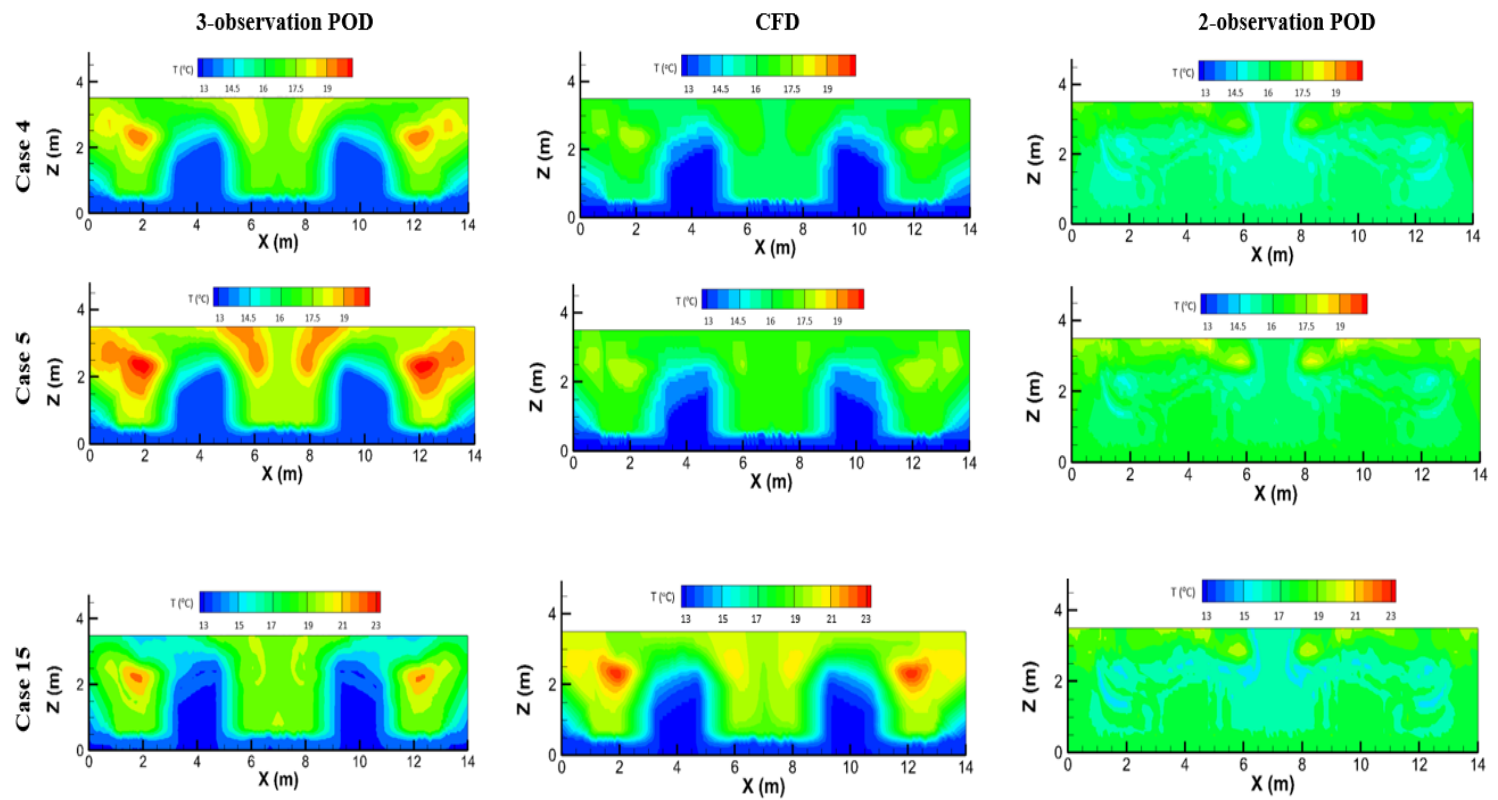

Fig. III.8 Comparison among 2-observation POD, 3-observation POD, and CFD results

Fig. III.9 shows the temperature profile of case 1 across one cold aisle and one hot aisle. Three scenarios are compared, i.e., the two-, three- observation, and the CFD cases. Three elevations of rack height, i.e. near bottom $(Z=0.65 \mathrm{~m})$, middle $(Z=1.60 \mathrm{~m})$, and near top $(\mathrm{Z}=2.55 \mathrm{~m})$, as previously indicated in Fig. III.2 are plotted. It is shown that the temperatures calculated from the three-observation basis functions agree with the original CFD case's results, while results that calculated from the two-observation basis functions deviates significantly, and in some locations, it can reach up to 3 centigrade. In the cold aisle, the air flow near the bottom and middle sections of the rack are almost identical, and 
it begins to fluctuate as it moves to the top of rack. Because of the strong cold jet from the perforated tiles in the cold aisle, the temperature profile is nearly constant at lower region of the cold aisle. However, at the top of the rack, cold air is mixed with hot air recirculated from hot aisle, which caused the temperature fluctuation. In the hot aisle, the air flow fluctuation occurs near the bottom and top of the rack height. Meanwhile, the air flow middle section of the rack is quite uniform. As a result, the temperatures observed in the corresponding sections tend to bear the same characteristics.

\subsection{Effects of Design Parameters on POD}

\subsubsection{Effect of Rack Inlet Air Temperature (Cases 1 - 5)}

Fig. III.10 shows the effect of rack inlet air temperature effect at the mid plane $(Y=5 \mathrm{~m})$. Only cases $1-3$ are shown due to limited space. Cold air plumes are clearly seen in these cases and gradually thinning out as the rack inlet air temperature increases. However, hot aisles are noticeably magnifying in the middle and on the two sides of the data center. The temperature difference between POD and CFD is, therefore, increasing, especially case 3. The temperature difference is proportional to the increase in the rack inlet air temperature. The increase with regard to Fig. III.6 is of linear relationship in the case of three-parameter observation scenario. The increase in rack inlet temperature from Table 3.2 is $0.3^{\circ} \mathrm{C}$ for each case. The sensitivity analysis suggests that for every $0.3^{\circ} \mathrm{C}$ of rack inlet air 
Cold

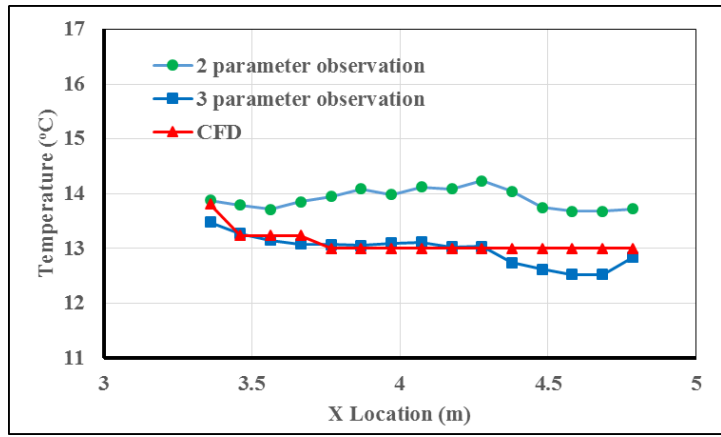

Top $(Z=2.55 \mathrm{~m})$

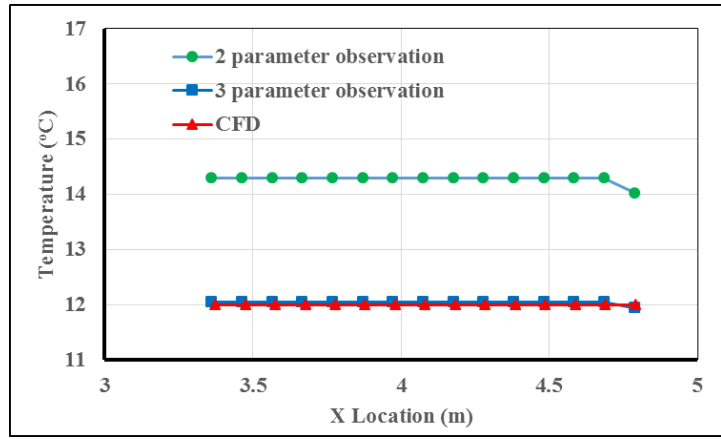

Middle $(\mathrm{Z}=1.60 \mathrm{~m})$

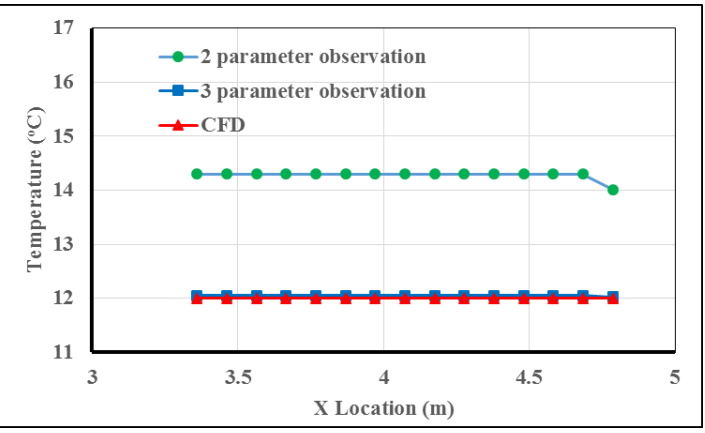

Bottom $(\mathrm{Z}=0.65 \mathrm{~m})$
Hot

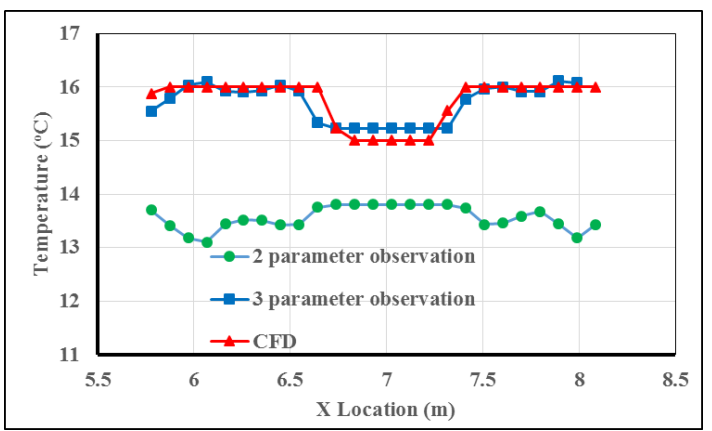

Top $(\mathrm{Z}=2.55 \mathrm{~m})$

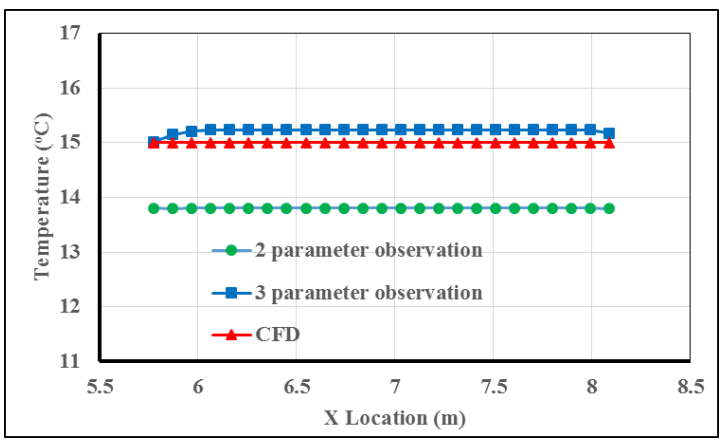

Middle $(\mathrm{Z}=1.60 \mathrm{~m})$

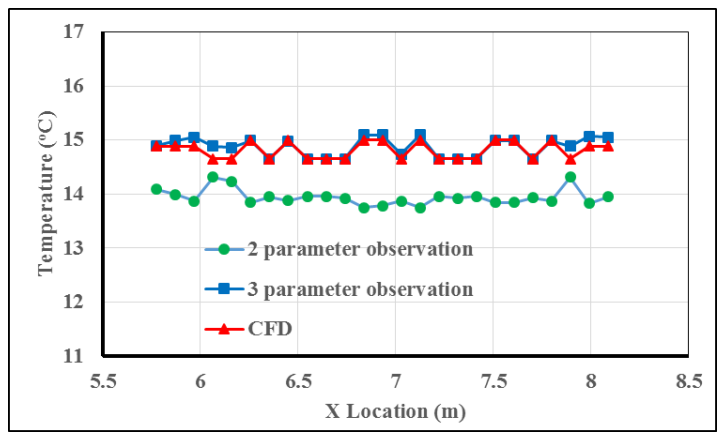

Bottom $(Z=0.65 \mathrm{~m})$

Fig. III.9 Temperature variation across cold (left) and hot (right) aisles for case 1

temperature increase, the temperature difference between POD and CFD is about $0.125^{\circ} \mathrm{C}$ rise. In all 3 cases, the cold aisles are almost unchanged in temperature. This is possibly 
because of the mesh density is much finer in these cold areas than anywhere else. The information is well retained and less likely to lose its quality as the POD process is performed.
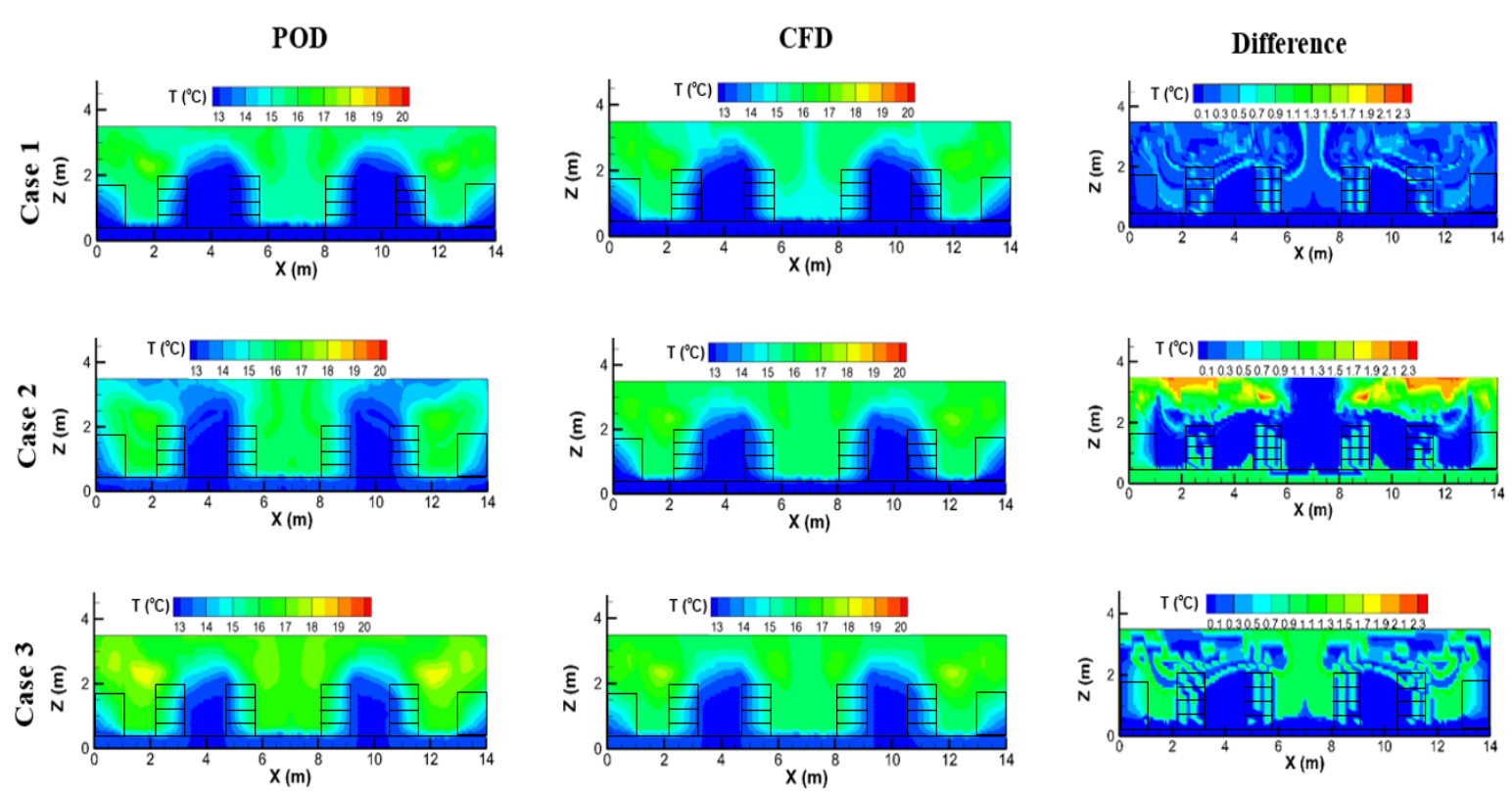

Fig. III. 10 CFD vs. POD, inlet temperature effect for case 1 - 3 (mid plane).

\subsubsection{Effect of Inlet Mass Flow Rate (Cases 6 - 10)}

Fig. III.11 shows the effect of mass flow rate on the temperature profile in the mid plane.

For the cases $8-10$, POD results tend to over predict the temperature obtained by CFD results. Indeed, the temperature difference rises as the mass flow rate increases. Although all 5 cases are not presented, from Fig. III.6, one can see that higher temperature difference is found in lower mass flow rate cases (cases $6 \& 7$ ). The difference is much profound in the ceiling area where return hot air rises. Unlike the effect of rack inlet air temperature, 
the underfloor plenum and cold aisles temperature obtained from POD method is quite different from CFD results. Case 9 with a mass flow rate of $7.1 \mathrm{~kg} / \mathrm{s}$ has the lowest temperature difference as compared to other cases. Since the temperature difference is fluctuating against the mass flow rate, sensitivity study is hardly carried out.
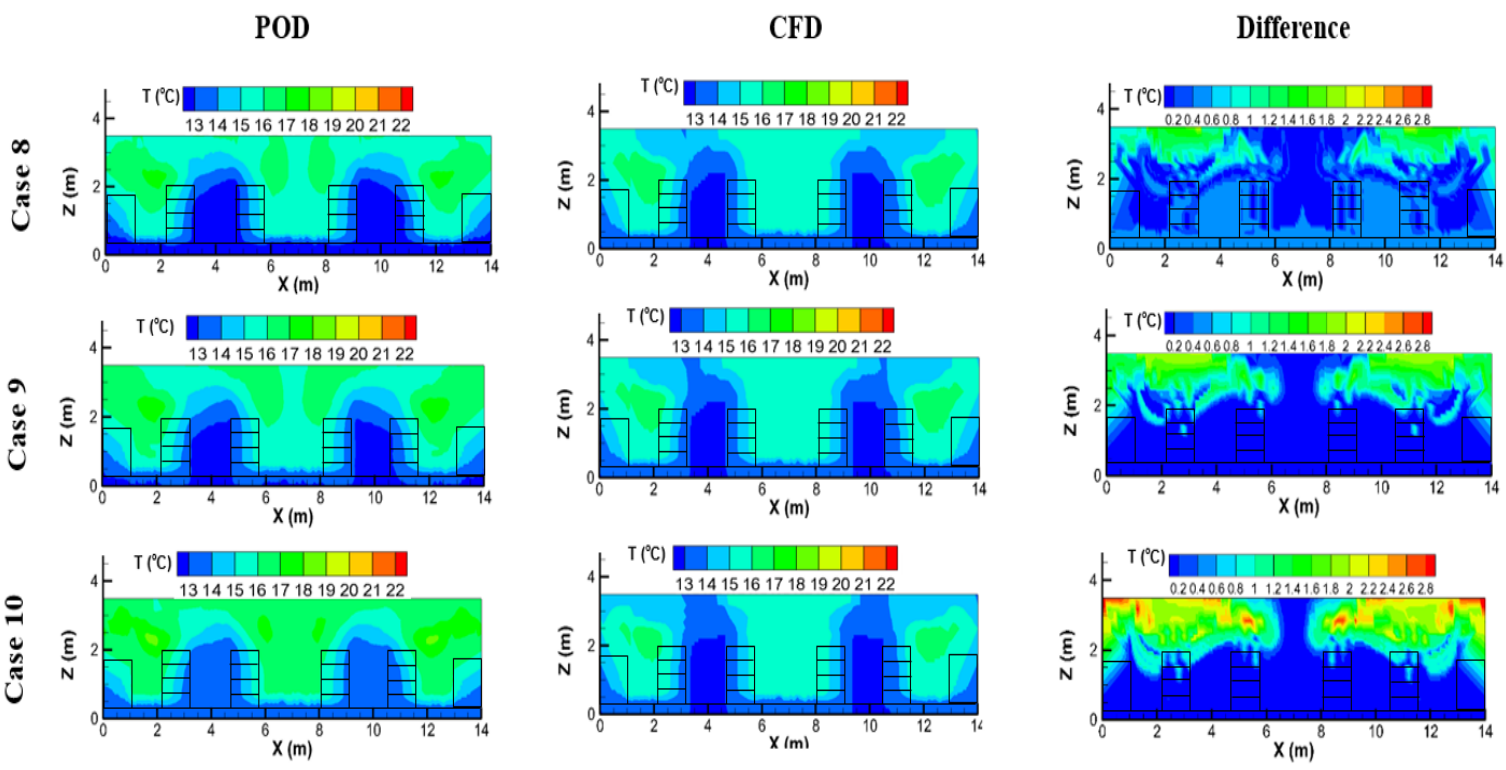

Fig. III.11 CFD vs. POD, mass flow rate effect for case 8 - 10 (mid plane).

\subsubsection{Effect of Server Heat Load (Cases 11 - 15)}

Fig. III.12 shows the effect of server load on the temperature profile in the mid plane. Only cases $11-13$ are shown due to limited space. The temperature difference is proportional to the increase in the server heat load. The increase with regard to Fig. III.6 is of linear relationship. The sensitivity analysis suggests that for every $1000 \mathrm{~W}$ of server heat load increase, the temperature difference between POD and CFD is about $0.5^{\circ} \mathrm{C}$ rise. Similar to 
the effect of mass flow rate, the difference is more profound in the region near the ceiling where hot air rises. The cold air plume remains unchanged throughout the parametric study as also observed in the effect of rack inlet air temperature cases.
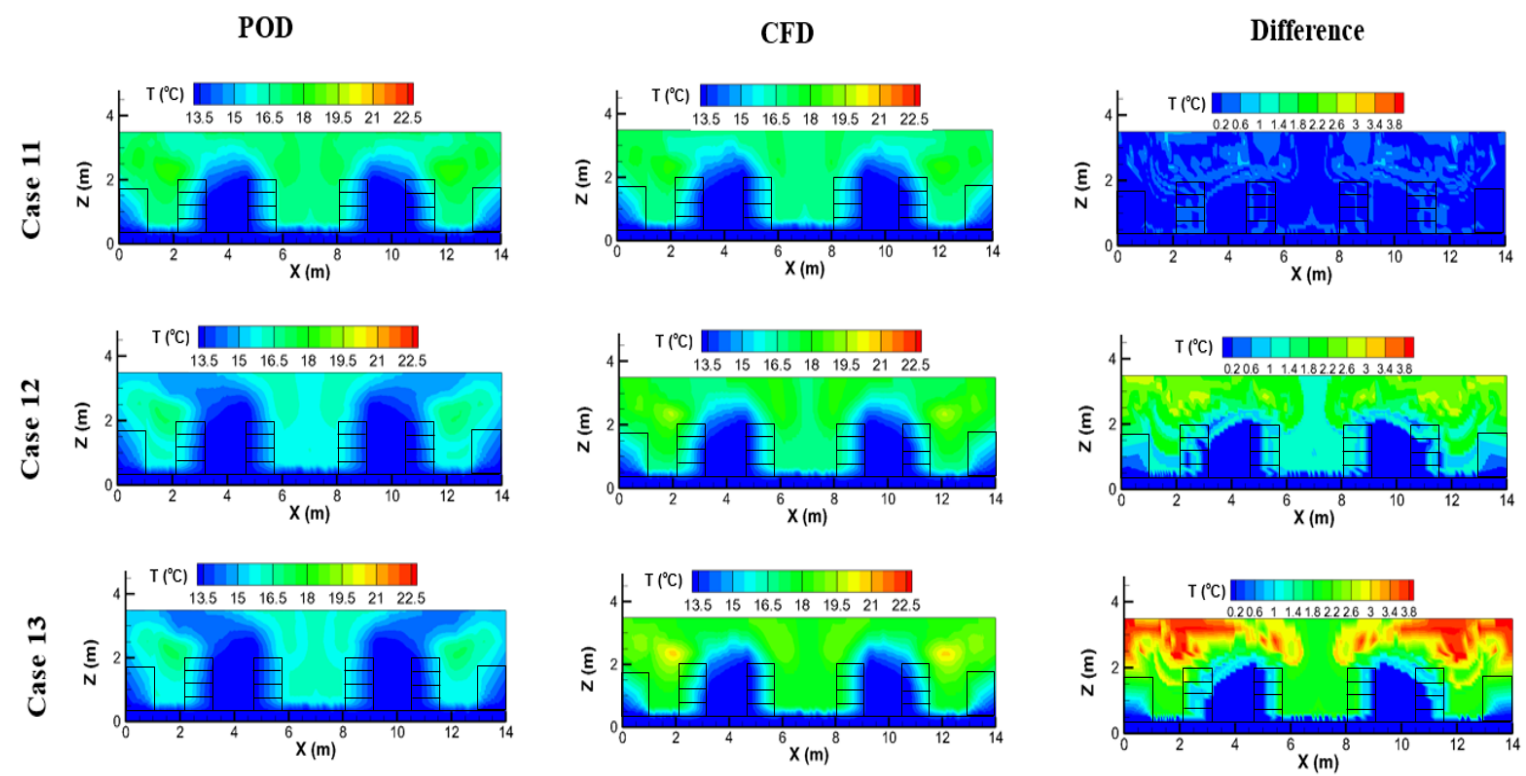

Fig. III.12 CFD vs. POD, server load effect for case $11-13$ (mid plane).

\subsection{3-D Thermal Reconstruction from 2-D}

Possible selected slides in the data center model helps to construct a 3D picture of the temperature profile. For the sake of method demonstration, three primary planes are presented. Since the geometry is symmetrical, only half domain is considered. The first selected plane is the mid plane parallel with the $\mathrm{XZ}$ surface at location $\mathrm{Y}=5 \mathrm{~m}$. This is the plane that has been analyzed throughout the current study. The second plane is right at the end of the server racks at location $\mathrm{Y}=2 \mathrm{~m}$., and the last plane is right at the face of one of the CRAC units. The hotspots are intense at the corners of the server racks facing hot aisles. 
As seen in Fig. III.13, the results from POD method are very close with the one obtained from CFD simulation.

Apparently, with POD method, potential hotspots can be detected with much less computational effort as compared to CFD simulation. In fact, for each CFD test case, it takes about 20 minutes to run, while it only takes about 2 seconds to process using POD method. During a design process, several test cases will be run to optimize the system with the most desirable design parameters. If we consider 100 more test cases will be run, then it takes about 1 day and 9 hours to complete in CFD and only 3 minutes in POD. That is 600 times faster if using POD method over CFD simulation. This makes POD method a perfect fit for real-time control management. In a real-life situation, a set of temperature sensors can be placed in a same surface feeding input data into a control box processed using POD method to give insights on temperature profile at the plane in question. The process continues at the surface moves across a dimension of the room to collect all the necessary information to form a 3D picture of the temperature profile of the data center. Control strategies need to be planned out to minimize the number of sensors and also the determination of sensor location. 


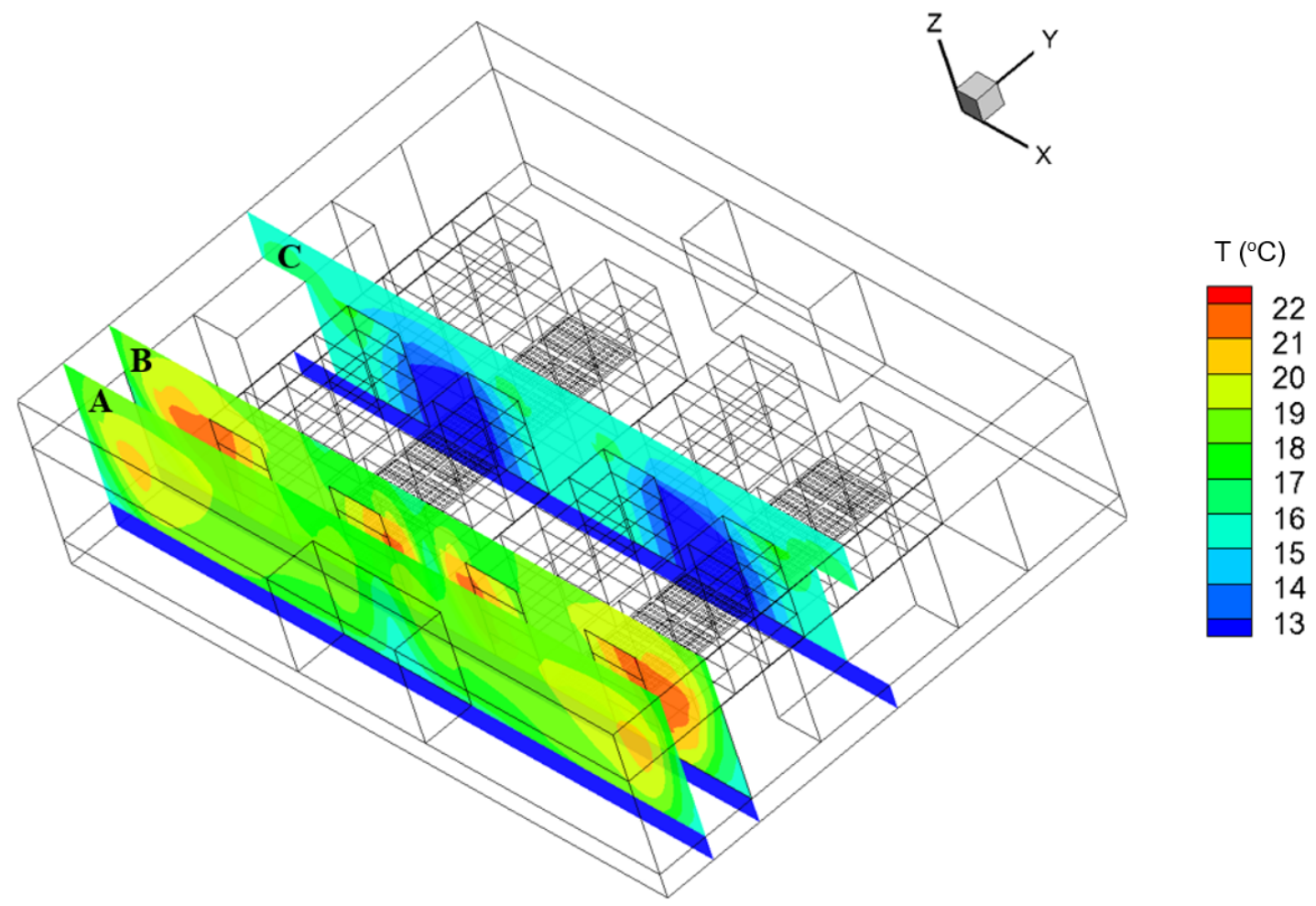

a) 3D layout

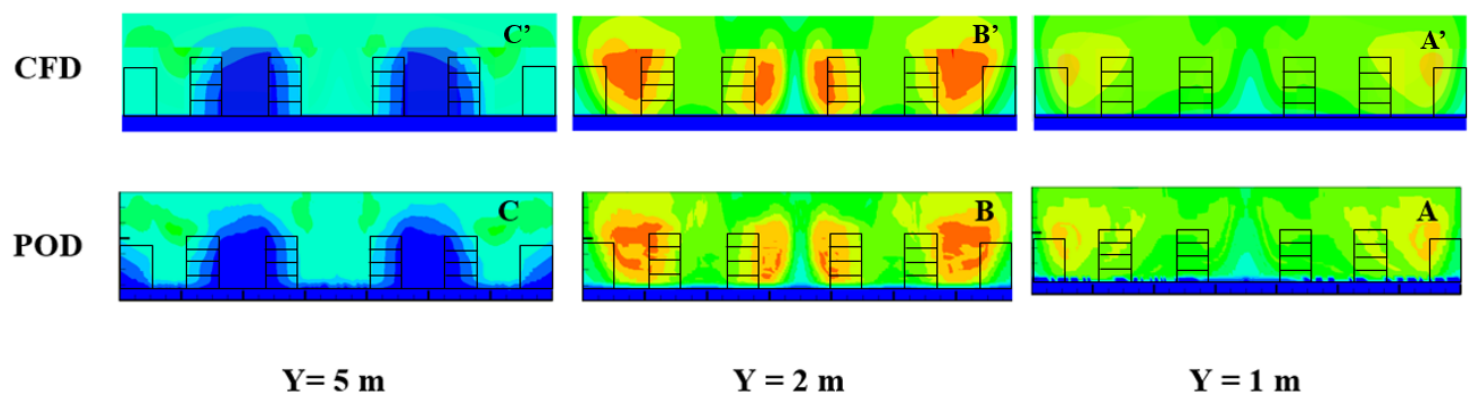

c) Temperature profile at different slices

Fig. III.13 Temperature profile for case 1. 


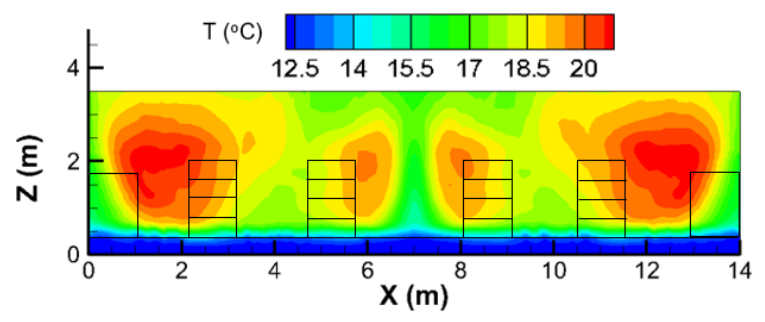

a) POD interpolation, $\mathrm{d}^{*}=0.1$

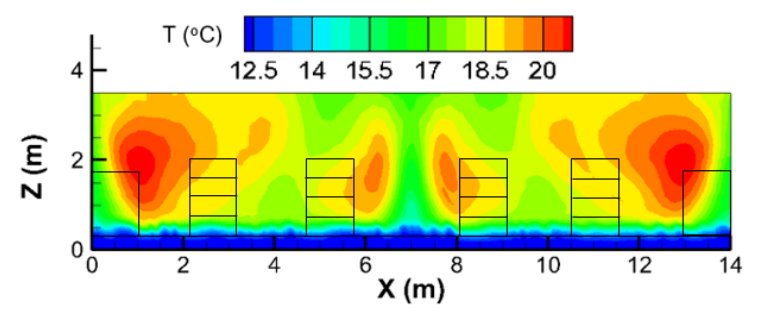

b) POD interpolation, $\mathrm{d}^{*}=0.02$

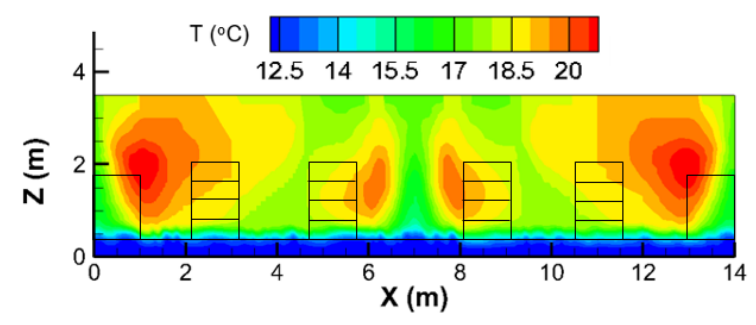

c) CFD

Fig. III.14 Temperature profile comparison at location $Y=1.5 \mathrm{~m}$ for different $d^{*}$

In order to test the accuracy of the interpolation technique for the POD model, temperature profile was investigated at location $\mathrm{Y}=1.5 \mathrm{~m}$. The plane locates at this location is called the target plane. Two-dimensional sections are generated along the $\mathrm{Y}$-axis direction. In the sensitivity analysis, a non-dimensional distance parameter $\left(\mathrm{d}^{*}\right)$, which is defined as the ratio of the distance between two selected (simulated) planes that contain the target plane to the entire width $(10 \mathrm{~m})$ of the data center model, is introduced. Two different values of $d^{*}(0.1$ and 0.02$)$ are studied in Fig. III.14 showing the comparison between the POD interpolation and the CFD data. For $\mathrm{d}^{*}=0.1$, the results are acceptable, though large discrepancies in some areas such as hot aisles, ceiling, and floor plenum are still profound. The maximum temperature difference is found to be $5.23^{\circ} \mathrm{C}(42.95 \%)$, while the minimum and average values are $3.2 \times 10^{-4 \circ} \mathrm{C}(0.00 \%)$ and $0.325^{\circ} \mathrm{C}(1.95 \%)$, respectively. Because of the large gap between plane A \& B, the discrepancy is generally large in some regions. 
As for $\mathrm{d}^{*}=0.02$, the accuracy is significantly improved since the distance to target plane is substantially narrowed. The maximum, average, and minimum temperature difference are drastically reduced to $0.565^{\circ} \mathrm{C}(3.29 \%), 0.165^{\circ} \mathrm{C}(0.97 \%)$, and $4.8 \times 10^{-5}{ }^{\circ} \mathrm{C}(0.00 \%)$, respectively. However, since the distance between two selected planes is inversely proportional to the number of planes to be simulated for a 3D reconstruction, a tradeoff between the number of simulated planes and the accuracy is clearly recognized. For instance, $\mathrm{d}^{*}=0.1$ needs totally 11 simulated planes, while $\mathrm{d}^{*}=0.02$ needs 51 planes. The choice of $\mathrm{d}^{*}$, thus, depends greatly on the compromise between the accuracy and speed of the simulation.

\subsection{Entropy Violation Check}

POD is known to have problems with satisfying locally second law of thermodynamics depending on the number of terms in the POD expansion [67]. For a sanity check, an entropy generation map is generated corresponding to 10 terms at a test location $\mathrm{Y}=2.0 \mathrm{~m}$. This location is situated right at the very end of the server rack rows. If the entropy generation is a non-negative quantity, the method shows not violation to the second law of thermodynamics; however, if it is negative, it has violated the law. It can be seen from Fig. III.15a, the original CFD has no entropy violation. However, when using POD to reconstruct the thermal profile of the data center, some violations can be spotted as seen in Fig. III.15b. These violations are mostly located in near the ceiling and not in the critical areas surrounding the server racks, other regions show no violation. Overall, the thermal 
reconstruction of the data center using POD is acceptable since there are not many cells violated the second law of thermodynamics.

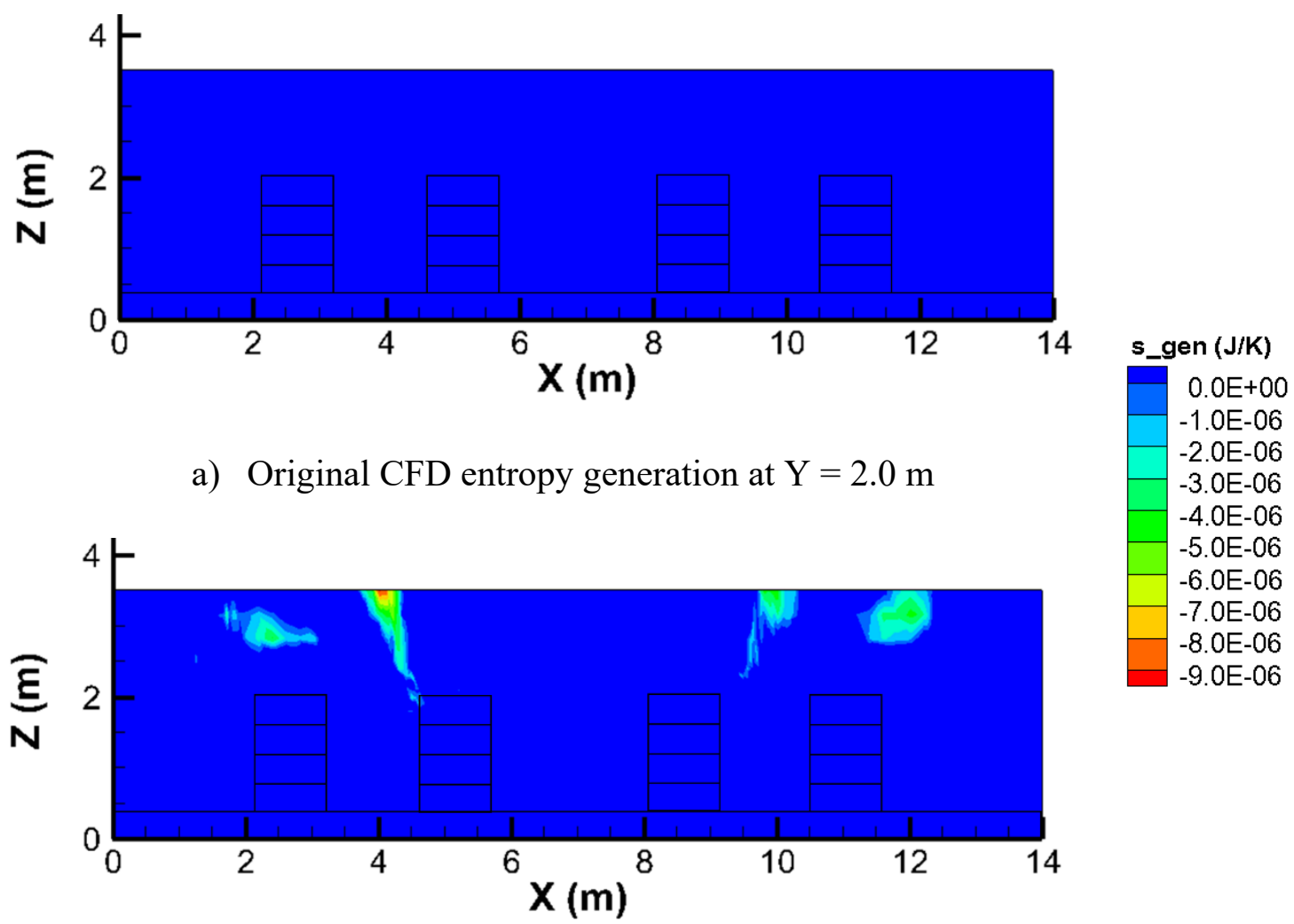

b) POD entropy generation for 10 modes at $\mathrm{Y}=2.0 \mathrm{~m}$

Fig. III.15 Entropy generation map for 10 modes

\subsection{Remarks}

In this chapter, proper orthogonal decomposition (POD) is presented as a new method that can be adopted for real-time convective system applications such as data centers. Fifteen test cases are tested against three different design parameters including rack inlet air temperature, inlet air mass flow rate, and server heat load. Moreover, the influence of the 
number of design parameter on POD basis functions is also considered. The conclusions of the current chapter are summarized as follows,

- Reduced order modeling methods are capable of drastically reducing the computational time of a normal full numerical solution from CFD/HT method. In the current study, it is found that POD is 600 times faster as compared to CFD/HT simulation

- The effect of each parameter shows the limitations of the POD method in the reconstruction of CFD model. There are almost no changes or minimal changes in the temperature difference in the case of rack inlet temperature variation, whereas it is a steady increase in the case of server heat load rises. The inlet mass flow rate condition, in contrast, peaks at lower and settles at higher flow rates.

- The three-parameter observation gives a smaller temperature difference in all the cases studied as compared to the two-parameter observation. This is an interesting finding regarding the effect of the number of observational parameters involved in the construction of the POD basis function.

- In addition, a 3-D thermal profile is created from selected primary planes and further information can be obtained by utilizing the interpolation method. A tradeoff between accuracy and number of primary planes is present for the current POD method. 
CHAPTER IV RESPONSE SURFACE METHODOLOGY 


\subsection{Response Surface Methodology (RSM)}

RSM has not been applied in a complex heat transfer system such as data centers. Therefore, it is of great interest for the authors to study the use of RSM for thermal management of data centers. Due to its rapid running time, RSM can be used to accommodate the optimization of multiple design parameters where thousands of virtual design scenarios need to be generated. In addition, RSM can also be applied to reconstruct the airflow and temperature fields quickly, which is potentially useful for real-time thermal monitoring of data centers. Four possible applications of RSM for data centers are introduced in this chapter. The first application focuses on finding an interpolation function that can be used to reconstruct the thermal profile within a data center model. Three different percentages of the original CFD data points $(5 \%, 10 \%$, and $20 \%)$ are compared with each other for training the response surface model in to get the overall temperature profile of a data center model. In second application, RSM model is applied with the goal of minimizing both the temperature difference of across the server racks and the maximum temperature within a data center model. The optimization process allows to seek the best design parameters that satisfy both of the said objectives. In the third application, the RSM approach is demonstrated with the objective of seeking the best tile porosity arrangement that can create a uniform airflow distribution through perforated tiles. In the fourth application, a prescribed airflow distribution of choice is obtained through the inverse design optimization, and three different scenarios including linear, parabolic, and sinusoidal tile airflow distributions are optimized with success. This could be particularly useful for modern data centers, which commonly undergo different non-uniform thermal loadings within server racks. In fact, as more advanced tiles with greater flexibility in 
porosity control are currently in use, the study could allow best practice in regard to perforated tiles placement for a more energy-efficient data center.

\subsubsection{Fundamentals of RSM}

Response surface methodology often starts with a database of training designs to construct for a response surface that fits through these designs by using an algorithm to guess the value of the unknown function on the basis of an assumption such as regularity, physical meaning, and statistical variability. RSM estimates the combination of the input parameters yielding an optimal response through fast-running approximation of the simulation process.

Response surface training is based upon a complete dataset, e.g., an evaluated design of experiment (DOE) information, experimental data, simulated results, etc. to create an approximation of the response in the design space. In this study, high-fidelity HT/CFD simulation data was used for training the response surface which was used later in the optimization procedure. Response surfaces are advantageous in problems where limited information is provided. However, the results obtained from these hyper-surfaces may be inaccurate if the design space exploration is poor and if an unsuitable method is chosen. Normally, the higher the number of training designs, the more accurate will be the RSM model. Furthermore, the design dispersion in the design space should be as uniform as possible for training a response surface. 


\subsubsection{Radial Basis Function (RBF)}

Some of the optimization techniques that may require thousands of objective functions calculation, and the objective functions may require hours or days to complete. Therefore, if one relies on traditional mesh-based methods, the calculations will be prohibited due to the cost of time required. The need for a simplified model or a metamodel that represent the original problem is indeed necessary. For instance, by using kernel interpolation/approximation technique, response surface methods which are based on linear and non-linear regression and other variants of the least square technique can be used to reduce a significant amount of time for those problems. These methods are often regarded as mesh-free methods.

One of the most popular mesh-free kernel approximation techniques is the one that uses radial basis functions (RBFs). Initially, RBFs were developed for multivariate data and function interpolation. It was found that RBFs were able to construct an interpolation scheme with favorable properties such as high efficiency, good quality and capability of dealing with scattered data, especially for higher dimension problems.

The radial basis functions typically have the following form

$$
S(\boldsymbol{P}) \cong \xi(\boldsymbol{x})=\sum_{i=1}^{N} w_{i} \phi(r)
$$

where $P=\left\{P_{1}, \ldots, P_{i}, \ldots, P_{L}\right)$ and $S(\boldsymbol{P})$ is known for a series of points $\boldsymbol{P}$. The approximating function $\xi(\boldsymbol{x})$ is represented as a sum of $\boldsymbol{N}$ radial basis functions, $\phi(r)$, where $r=\left|\boldsymbol{x}-\boldsymbol{x}_{\boldsymbol{i}}\right|$. Each RBF is associated with a different center $\boldsymbol{x}_{\boldsymbol{i}}$, and weighted by an appropriate coefficient, $w_{i}$. The weights $w_{i}$ can be computed by using the matrix methods of linear 
least squares. Since the values of $\boldsymbol{P}$ points are known, they can be substituted and eq. (IV.1) is now rewritten in the matrix form.

$$
\Phi W=\Xi
$$

where unknowns are the weight matrix $\boldsymbol{W}$, and $\boldsymbol{\Phi} \& \boldsymbol{\Xi}$ are the basis and approximation function matrices, respectively. Once all the weights are known, they can be put back into eq. (IV.1) to obtain the approximate function $\xi(x)$. In order to solve for the weight matrix, $\boldsymbol{W}$, from eq. (IV.2), matrix $\boldsymbol{\Phi}$ needs to be inverted as shown in eq.(IV.3).

$$
W=\Phi^{-1} \Xi
$$

In multi-dimensional problems, $r$ is the distance between two points. RBFs are special functions that have a finite value for $r=0$, and tends to zero as $r$ goes to infinity. These functions are called globally supported. Some of the globally supported RBFs used in this chapter are shown in eqns. (IV.4) - (IV.7) of Table IV.1. The shape parameter $c_{j}$ is kept constant as $1 / \mathrm{N}$. It is used to control the smoothness of the RBF. Fig. IV.1 shows the influence on its choice for the multiquadrics RBF. An increase in c results in a smoother curve for RBF.

Table IV.1 Choices for basis functions

\begin{tabular}{|c|c|c|}
\hline \multicolumn{1}{|c|}{ Multiquadrics } & $\phi\left(\left|x-x_{i}\right|\right)=\sqrt{\left(x-x_{i}\right)^{2}+c_{i}^{2}}$ & (IV.4) \\
\hline Inverse Multiquadrics & $\phi\left(\left|x-x_{i}\right|\right)=\frac{1}{\sqrt{\left(x-x_{i}\right)^{2}+c_{i}^{2}}}$ & (IV.5) \\
\hline Gaussian & $\phi\left(\left|x-x_{i}\right|\right)=e^{-c\left(x-x_{i}\right)^{2}}$ & (IV.6) \\
\hline Polyharmonic splines & $\phi\left(\left|x-x_{i}\right|\right)=\left\{\begin{array}{l}\left(x-x_{i}\right)^{2 n} \log \left(x-x_{i}\right), n \geq 1, \text { in 2D } \\
\left(x-x_{i}\right)^{2 n-1}, n \geq 1,\end{array}\right.$ & in 3D \\
\hline
\end{tabular}




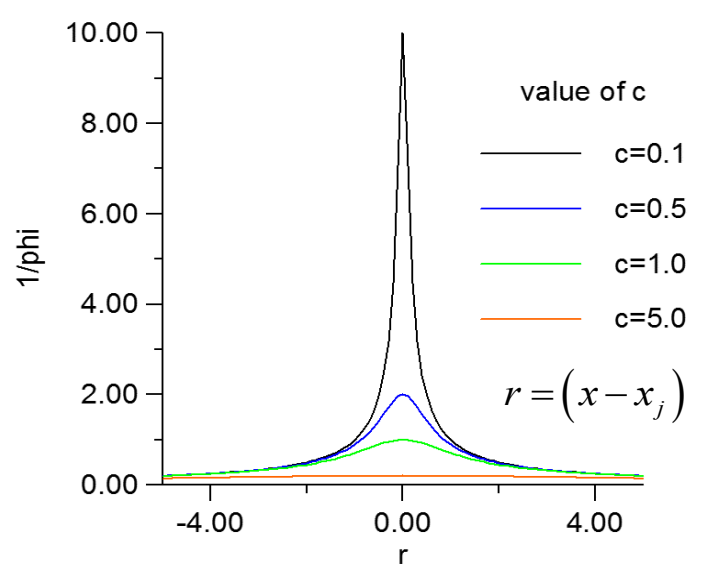

Fig. IV.1 The influence of shape parameter $c_{j}$ on multiquadrics RBF [18]

\subsubsection{RSM Performance Criteria}

RSM accuracy depends on several factors such as the complexity of the variations of the solution, the number of points in the original DOE and the choice of the type of RSM. Since an RSM model is typically obtained through an approximation process, it is very important to assess its quality with respect to the available real designs for reasonable accuracy. Multiple RSMs for the same output variable should be created and compared against one another before choosing the best one. If the quality is unsatisfactory, the

process should be restarted, and further experiments should be made. In any case the described procedure should be iterated several times in order to obtain the best possible results. To provide a more complete picture of metamodel accuracy, three different metrics are often used: $R$-square, Relative Average Absolute Error (RAAE), and Relative Maximum Absolute Error (RMAE) [38]. 
R Square

$$
R^{2}=1-\frac{\sum_{i=1}^{n}\left(y_{i}-\hat{y}_{i}\right)^{2}}{\sum_{i=1}^{n}\left(y_{i}-\bar{y}\right)}=1-\frac{M S E}{\text { variance }}
$$

where, $\hat{y}_{i}$ is the corresponding predicted value for the observed value $y_{i} ; \bar{y}$ is the mean of the observed values. A larger value of $R$-square indicates a more accurate metamodel.

Relative Average Absolute Error

$$
R A A E=\frac{\sum_{i=1}^{n}\left|y_{i}-\hat{y}_{i}\right|}{n * S T D}
$$

where $S T D$ stands for standard deviation. RAAE shows the relative average absolute error calculated for $n$ values. The smaller the value of $R A A E$, the more accurate the metamodel.

Relative Maximum Absolute Error

$$
R M A E=\frac{\max \left(\left|y_{1}-\hat{y}_{1}\right|,\left|y_{2}-\hat{y}_{2}\right|, \ldots,\left|y_{n}-\hat{y}_{n}\right|\right)}{S T D}
$$

Large $R M A E$ indicates large error in one region of the design space even though the overall accuracy indicated by $R$-square and $R A A E$ can be very good. Therefore, a small $R M A E$ is preferred. However, since this metric cannot show the overall performance in the design space, it is not as important as $R$-square and $R A A E$. 


\subsection{Data Center Models}

\subsubsection{Data Center Model 1}

In this section, the data center model was adopted from the one that has been used by previous studies, [4], [48], [49], [61]. Refer to Fig. II.1 and Fig. III.2 for the schematic of the model and the hot and cold aisle configuration, respectively. The detailed descriptions of this model are outlined in section 3.1.2.a of Chapter 3. The numerical method used can be referred section 3.1.1.b. The first two RSM applications utilize this data center model for the study. The first application demonstrates how to use RSM for reconstructing the thermal profile, and the second application focuses on the multi-objective optimization for optimal design input of a data center model. The whole process of the CFD-based RSM modeling process is shown in the following flowchart.

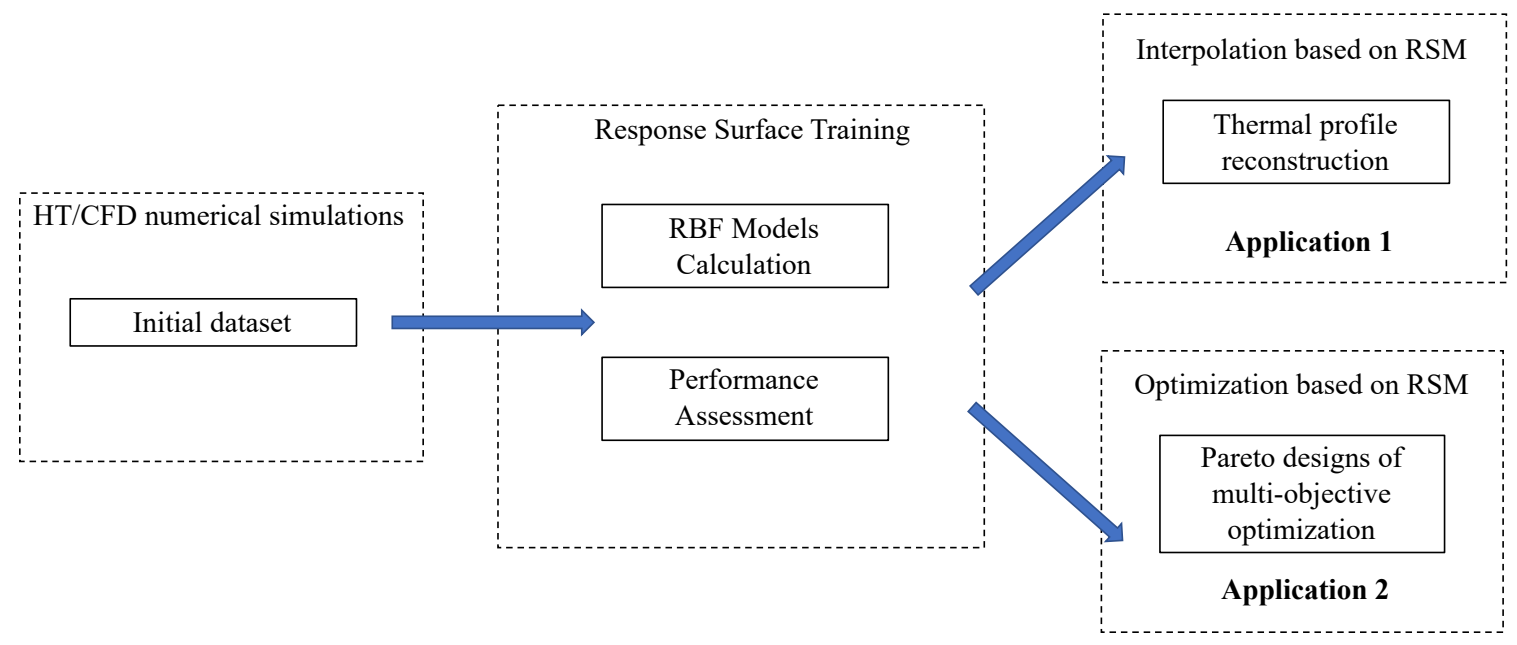

Fig. IV.2. CFD-based RSM used in two applications for a data center model 


\subsubsection{Data Center Model 2}

\section{a. Descriptions}

The data center model used in this study is modeled after the Data Center Laboratory (DCL) at Georgia Tech which also used in previous studies, [8], [9], [41]. The dimension of the data center model in $\mathrm{L} \times \mathrm{W} \times \mathrm{H}$ is $28.5 \mathrm{ft} . \times 21.3 \mathrm{ft} . \times 8.5 \mathrm{ft} .(8.7 \mathrm{~m} \times 6.2 \mathrm{~m} \times 2.6$ m). Three CRAH units placed in the room extend from the floor all the way to the ceiling. CRAH $1 \& 2$ are down-flow units while CRAH 3 is the only up-flow unit. However, in this study, only CRAH 1 was utilized, and other two were blocked off. Two additional units with an equal height of $5.68 \mathrm{ft} .(1.73 \mathrm{~m})$ placed in the room are the power distribution unit (PDU) and the coolant delivery unit (CDU). The standard 42U server rack is filled with four $10 \mathrm{U}$ rack-mounted server simulators. Each server simulator model has a dimension of 24 in. $(60.96 \mathrm{~cm})$ wide, 18 in. $(45.72 \mathrm{~cm})$ high, and $27.125 \mathrm{in} .(69 \mathrm{~cm})$ deep. There is a 6 in. $(15.24 \mathrm{~cm})$ gap at the foot of the server rack.

The server racks are arranged in such a way that the front-door sides facing each other form a cold aisle. This cold aisle is where the two rows of standard $2 \times 2 \mathrm{ft}^{2}$ perforated tiles are positioned to supply cooled air to the server racks. The back sides of the server racks exhaust heat from active servers by internal fans to form hot aisles as seen in Fig. IV.3. The underfloor plenum has a height of $3 \mathrm{ft}$. (0.91), which is relatively deep compared a regular 2-foot raised floor industry standard. The ceiling plenum height is $3 \mathrm{ft} .(0.91 \mathrm{~m})$. The ceiling vents are located along the walls of the hot aisle sides (refer to Fig. IV.4). 
$28.5 \mathrm{ft} .(8.7 \mathrm{~m})$

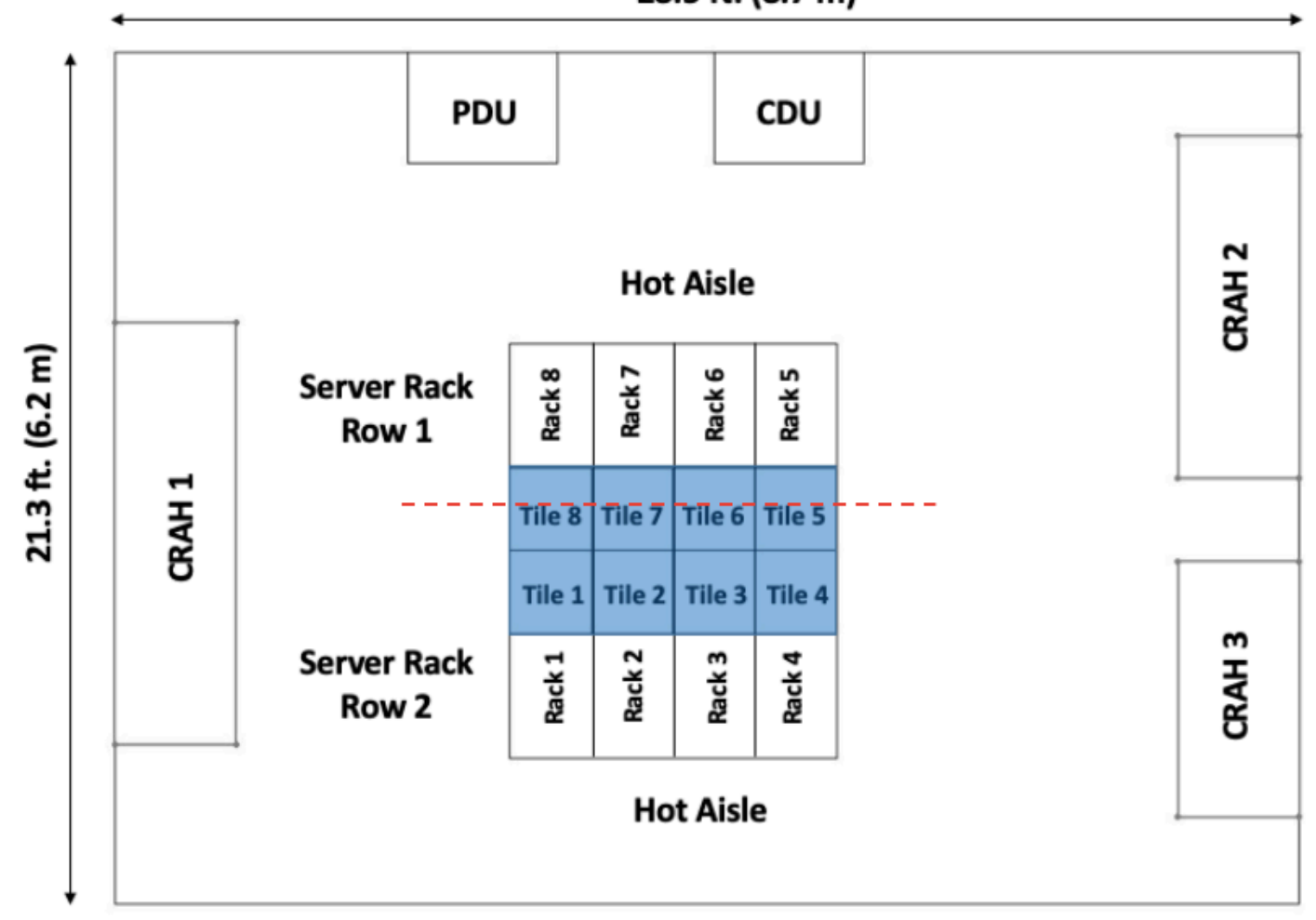

Fig. IV.3. A simplified data center overview floor plan with floor tiles shown

\section{b. Computational Model}

The computational domain of the current server model is shown in Fig. IV.4. The downflow CRAH unit number one located right in the middle of the cold aisle was modeled as a black box, i.e., only mass flow inlet was specified at the bottom surface of the CRAH. The other two CRAH units were not considered in this study, and therefore, only serves as space fillers. The supply airflow from CRAH unit number one was assumed to be normal to the surface of discharge. The ceiling vents were treated as pressure outlets. Two rows of server racks with a total of 32 server simulators with each server was modeled as a black box. Since there are total 4 server racks in each row and 4 server simulators in each rack, both 
the front and back sides of each row were divided in 16 smaller windows representing 16 server simulator cabinets. Server rack thermal load is not considered in this study because only airflow modeling is investigated. Due to the nature of a black box model, thirty-two small surfaces facing the cold aisle were treated as outflow, while 32 exhaust surfaces facing the hot aisles were modeled as inflow. The perforated floor tile flow model used was the modified body force model [8] with a six-inch enhanced momentum region above all eight perforated tiles. This tile model has extensively been validated with high fidelity. The three-foot raised floor plenum was included in the domain without any stanchions, pipes, or cables explicitly modeled, while the ceiling plenum was disregarded.

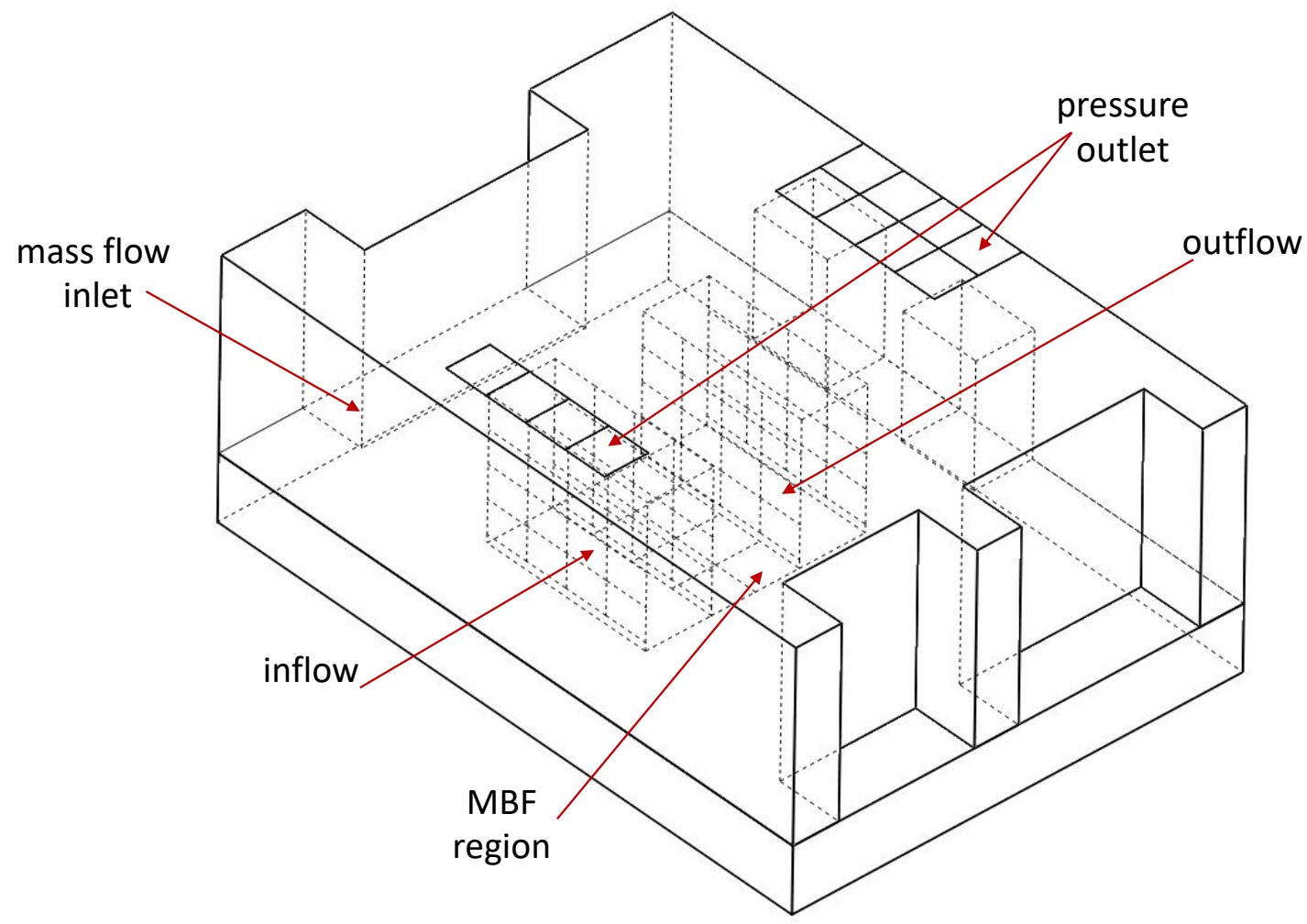

Fig. IV.4. Computational domain and boundary conditions 


\section{c. Numerical Model}

Standard $k$ - $\varepsilon$ turbulence model [44] was utilized in the numerical process using a commercial code [6]. Majority of the meshes having $y+$ greater than 30 , and enhanced wall treatment was deployed to ensure numerical stability for a wider range of mesh. The convergence criteria were set to be less than $10^{-4}$ in residuals for all computed variables. In some cases where set criteria could not be reached, the quantity of interests were monitored to stabilize to a constant value within less than $1 \%$ of a difference for a convergence to be reached. Since only airflow simulation is performed, the energy equation was not solved in this study. The gradients in spatial discretization were selected as least square cell based. The pressure-velocity coupling scheme used was the SIMPLE algorithm. Turbulent kinetic energy and turbulent dissipation rate equations were solved by the first order upwind scheme, while momentum equation was used with the second order upwind scheme.

Since the server rack model consisted of mostly orthogonal features, it was therefore meshed with a cut-cell method where hexahedral mesh is dominant. Grid independence study was performed to ensure an accurate result yet less computing time. The typical velocities along the probing line located at 6 feet above the centerline of the perforated tiles 5 through 8 (refer to Fig. IV.3) for different mesh sizes are shown in Fig. IV.5. It is concluded that at 1.7 million cells, the integrity of the tile velocity is preserved, and a further increase in grid size would not justify more computing time. 


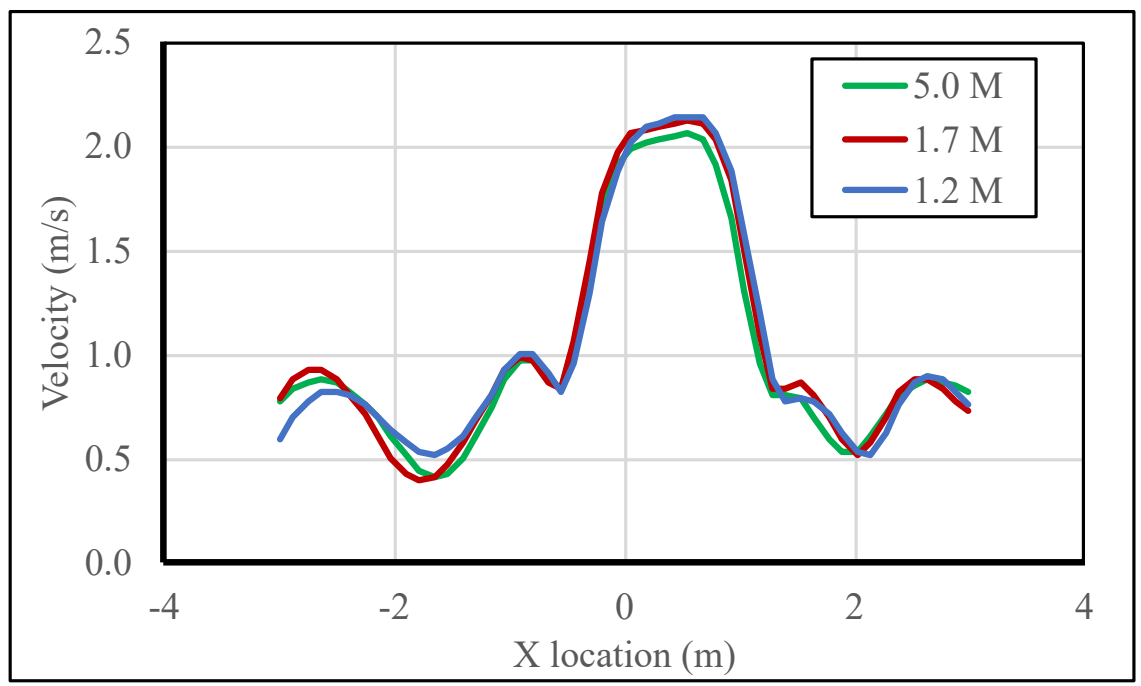

Fig. IV.5. Velocities along the probing line for different mesh sizes

Table IV.2. Attributes of the genetic algorithm used during the optimization process

\begin{tabular}{|l|c|}
\hline Number of design variables & 8 \\
\hline Initial population size & 45 \\
\hline Number of generations & 100 \\
\hline Probability of Mutation & $1.5 \%$ \\
\hline Probability of Elitism & $3 \%$ \\
\hline Probability of directional crossover & $50 \%$ \\
\hline
\end{tabular}

\section{d. Tile Airflow Uniformity and Inverse Design Optimization}

ModeFrontier optimization program [25] was used for demonstrating the RSM method. The optimization was carried out utilizing the genetic algorithms (GAs) based entirely on RSM for calculation of the generations. GAs are based on the principles of natural genetics and natural selection. It is suitable for the indoor environment of building optimization since it is a gradient-free stochastic optimization method. Compared with other stochastic 
search methods, GAs have the features of robustness and effectiveness even in noisy environments [46]. The basic elements of natural genetics - reproduction, crossover, and mutation - are implemented during numerical optimization. Table IV.2 summarizes all of the attributes considered during a GA process.

The general form of the objective function is shown in eq. (IV.11).

$$
\dot{\boldsymbol{M}}(\boldsymbol{T}) ; \boldsymbol{T}=\left\{T_{1}, T_{2}, \cdots, T_{L}\right\}
$$

where $T_{1}, T_{2}, \cdots, T_{L}$ are the tile porosities, which can be modified in order to find the minimum value of the function $\dot{\boldsymbol{M}}$.

$$
\dot{\boldsymbol{M}}= \begin{cases}\sqrt{\frac{\sum_{i=1}^{N}\left(\hat{\dot{m}}_{i}-\overline{\dot{m}}\right)^{2}}{n},} & \text { uniformity design } \\ \sqrt{\frac{\sum_{i=1}^{N}\left(\hat{\dot{m}}_{i}-\dot{m}_{i}\right)^{2}}{n},} & \text { inverse design }\end{cases}
$$

In the tile airflow uniformity design optimization, the objective function $(\dot{\boldsymbol{M}})$ is essentially the standard deviation between the predicted tile mass flow rates by RSM, $\widehat{\dot{m}}_{i}$, and the average value, $\overline{\dot{m}}$. In the inverse design optimization, the objective function $(\dot{\boldsymbol{M}})$ is the root mean square error between the predicted tile mass flow rates by RSM, $\widehat{\dot{m}}_{i}$, and the CFD values, $\dot{m}_{i}$. In both cases, $n$ represents the number of tiles.

\subsection{RSM-based applications}

\subsubsection{Application 1: Data Center Model Reconstruction Using RSM}

In this section, we explore how CFD-based RSM helps in reconstructing the thermal profile for a much smaller original dataset compared to the full HT/CFD results. Extendedly, this is particularly helpful when applied to a sensor-based RSM in the event of real-time 
thermal monitoring of a data center. By utilizing an adequate number of sensors, one can approximate a function based on RSM that can interpolate other thermal points within a data center

\section{a. RBF-based response surface training}

The response surface is trained using the training points obtained from coordinates of each cell within the domain. In real life situation, these coordinates point to the locations of sensor temperature probes. The more training points or sensor locations the more accurate the trained response surface is. However, sometimes it is very expensive to afford many data points in experiments. Another requirement is the input parameters space is uniformly explored in the range of variations of the input parameters to obtain a more reliable response surface. Here, the input parameters will be $\mathrm{x}, \mathrm{z}$ coordinates, and the output (response) parameter is temperature. The data table including both inputs and outputs is split into two categories (training \& validation) by a prescribed percentage.

In this application, we investigate three different scenarios of training data points, namely, $5 \%$ ( 237 points), $10 \%$ (473 points), and 20\% (945 points) of the original data table (4723 points). Basically, a seed algorithm to randomly sample the data points is used. Seed is a positive integer number (from 0 to 1000) used as the starting point for generating the sequence of random numbers. The same seed reproduces exactly the same sequence. If the seed value is 0 , the sequence is automatically seeded to a value based on the current time. 
The sequence is then used to select the training points. Fig. IV.6 shows how uniformly the points are distributed in the design space for response surface training.

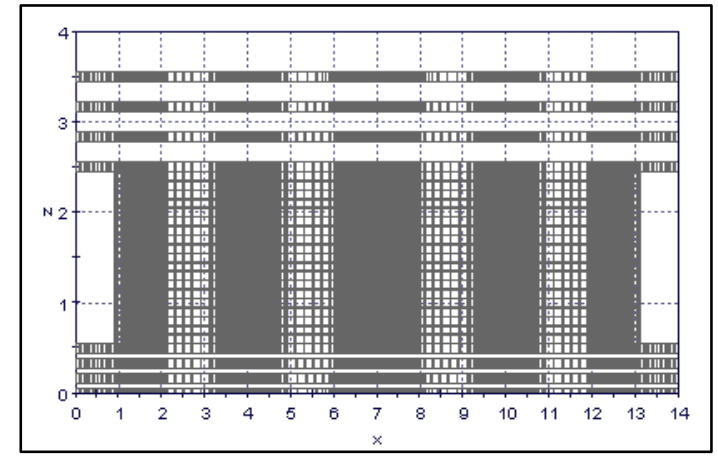

a) Original data points (4723)

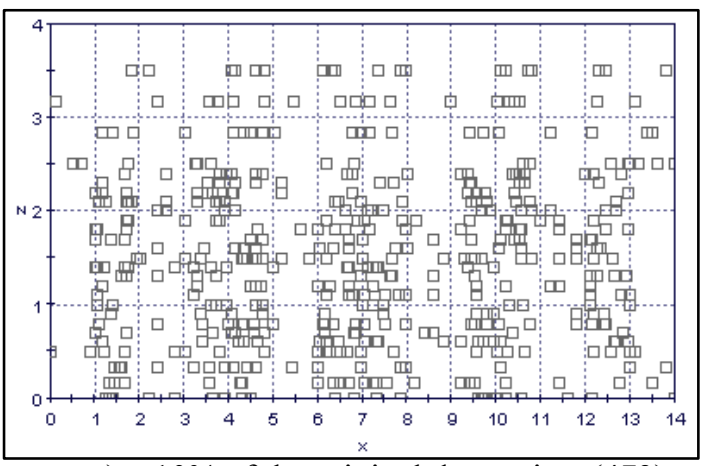

c) $10 \%$ of the original data points (473)

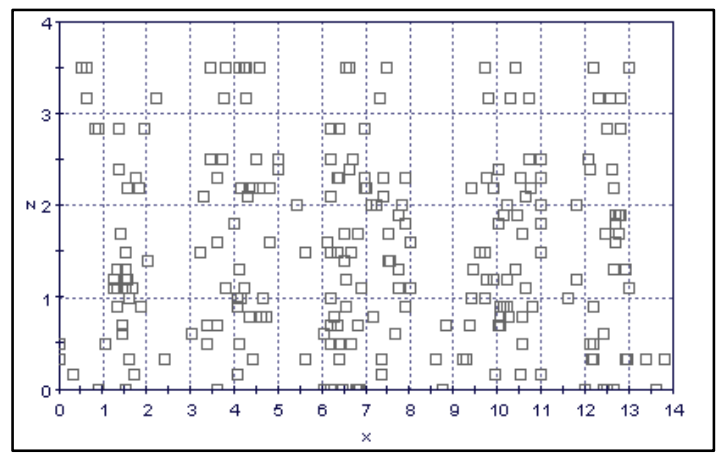

b) $5 \%$ of the original data points (237)

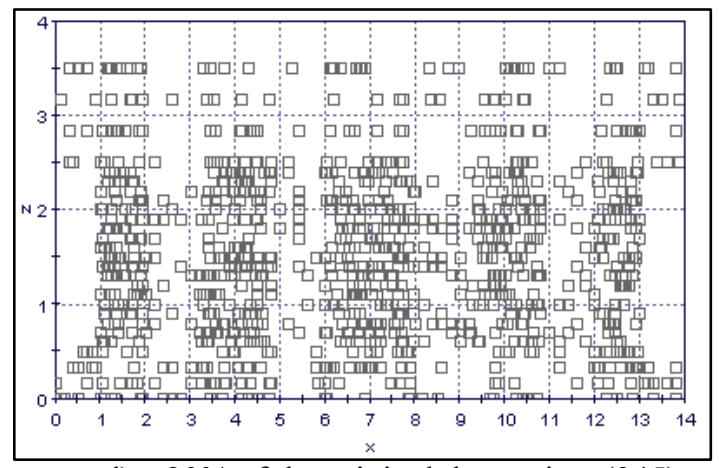

d) $20 \%$ of the original data points (945)

Fig. IV.6. Random samples of 5\%, 10\%, and 20\% of the original dataset

The RBF models (eq. (IV.4) - (IV.7)) are analyzed based on assessment criteria stated in eq. (IV.8) - (IV.10). The quality of the response surface relies on these assessments. As seen in Table IV.3, the polyharmonic spline RBF (eq. (IV.7)) consistently dominates other RBF models in the response surface training throughout three scenarios $(5 \%, 10 \%$, and $20 \%$ training points). It successfully passes the performance criteria with high fidelity. Although the multiquadric RBF has the three metrics comparable to the polyharmonic spline RBF, it is not chosen as the basis function for constructing response surface in this 
study. As stated before, the two most important performance assessment criteria are $R^{2}$ and RAAE. As seen in Table IV.3, multiquadric RBF has higher RAAE in the case of $20 \%$ training points, which makes it less desirable to use. Other RBF models show inferior performance as compared to multiquadric and polyharmonic spline RBFs.

Table IV.3. Performance measurement among different RBF models

\begin{tabular}{|c|c|c|c|c|c|c|c|c|c|}
\hline \multirow{2}{*}{ RBF Models } & \multicolumn{3}{|c|}{ R square } & \multicolumn{3}{c|}{$\boldsymbol{R A A E}$} & \multicolumn{3}{c|}{$\boldsymbol{R M A E}$} \\
\cline { 2 - 10 } & $20 \%$ & $10 \%$ & $5 \%$ & $20 \%$ & $10 \%$ & $5 \%$ & $20 \%$ & $10 \%$ & $5 \%$ \\
\hline Multiquadrics & 0.9501 & 0.9601 & 0.9559 & 0.1399 & 0.0837 & 0.1016 & 3.1458 & 2.0396 & 2.0371 \\
\hline $\begin{array}{c}\text { Inverse } \\
\text { Multiquadrics }\end{array}$ & 0.2185 & 0.9452 & 0.9525 & 0.0961 & 0.1020 & 0.1091 & 21.3815 & 2.5509 & 2.0755 \\
\hline Gaussian & 0.7403 & 0.9036 & 0.8056 & 0.1090 & 0.2088 & 0.2424 & 12.2094 & 3.0399 & 2.3340 \\
\hline $\begin{array}{c}\text { Polyharmonic } \\
\text { Spline }\end{array}$ & 0.9623 & 0.9524 & 0.9554 & 0.0475 & 0.0767 & 0.1040 & 2.6154 & 2.3691 & 2.0453 \\
\hline $\begin{array}{c}\text { Wendland's } \\
\text { form }\end{array}$ & 0.9468 & 0.9231 & 0.9473 & 0.0567 & 0.1034 & 0.1103 & 3.6419 & 2.5862 & 2.0556 \\
\hline
\end{tabular}

Fig. IV.7 shows the temperature output computed from the validation table for two best and worst RBFs in the case of using $20 \%$ training points from the original CFD data. Ideally, if a response surface is able to identify the behavior of the system, the temperature output computed by the response surface will coincide with the original CFD temperature data. Therefore, a diagonal line of approximately $45^{\circ}$ is plotted. In Fig. IV.7, real and virtual points represent the original CFD temperature points and the calculated temperature from validation table based off the constructed response surface.

Fig. IV.8 shows three types of residual calculated from the validation table for the case of $20 \%$ training point utilization. The area where most error occurs is within the first 1000 data points. The maximum absolute error is about $4 \mathrm{~K}$, while the maximum relative error is approximately $1.5 \mathrm{~K}$ 


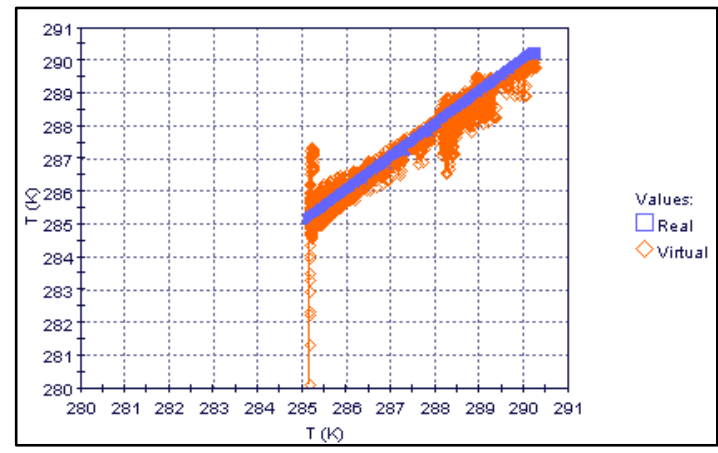

a) Multiquadric RBF (20\% training points of the original data)

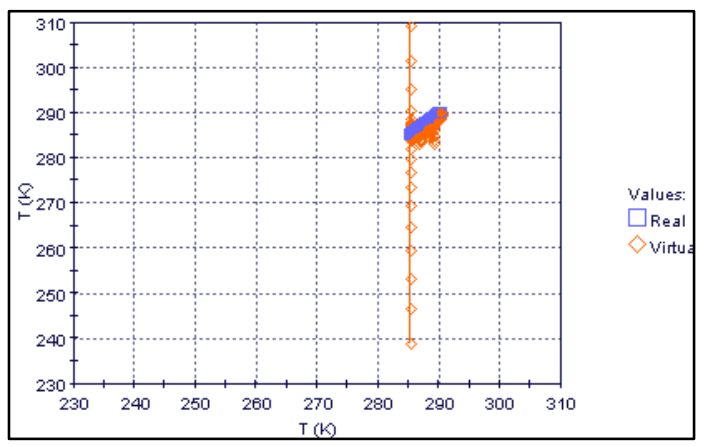

c) Inverse Multiquadrics RBF (20\% training points of the original data)

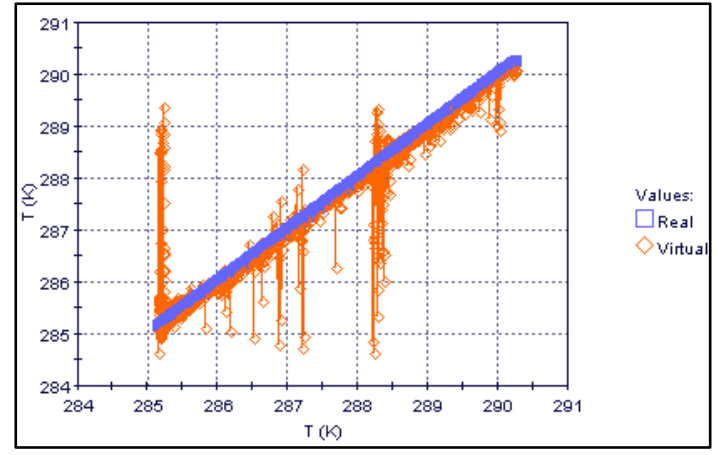

b) Polyharmonic Spline RBF (20\% training points of the original data)

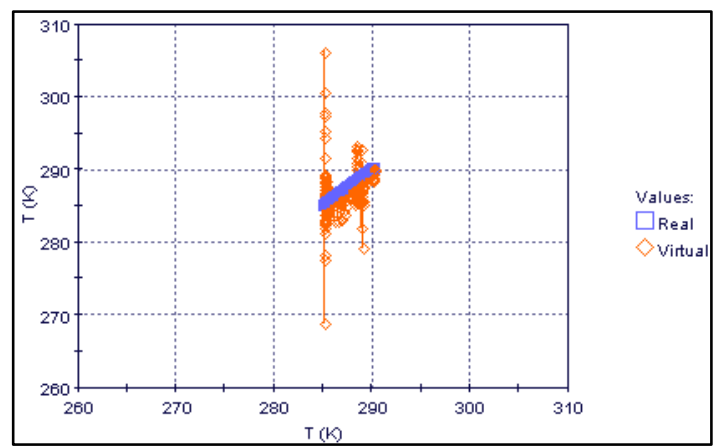

d) Gaussian RBF (20\% training points of the original data)

Fig. IV.7. Distance chart comparison among 4 different RBF models

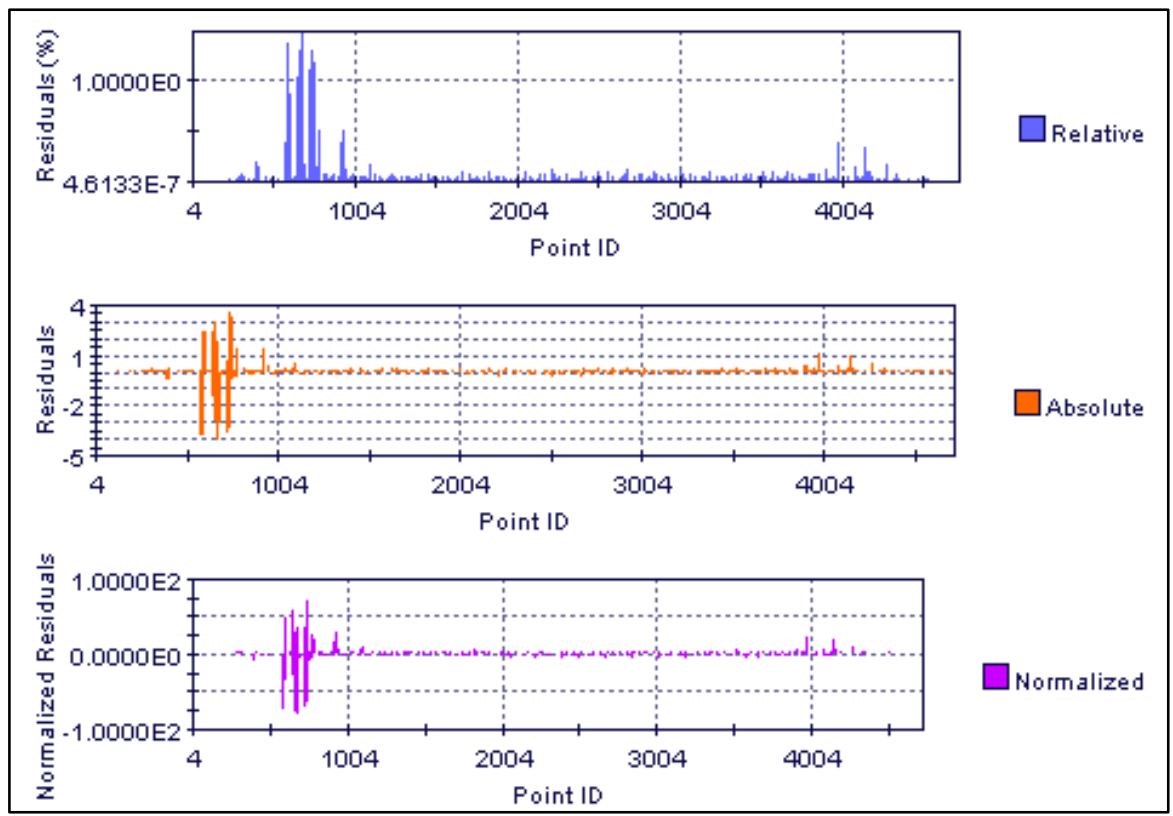

Fig. IV.8. Residual chart for Polyharmonic Spline 


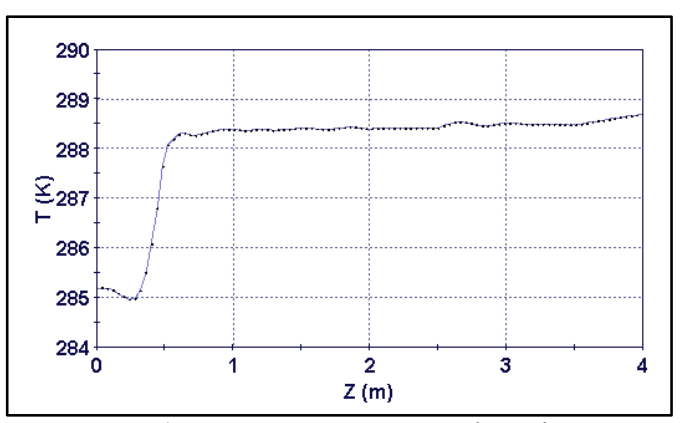

a) Temperature vs. $z$ location

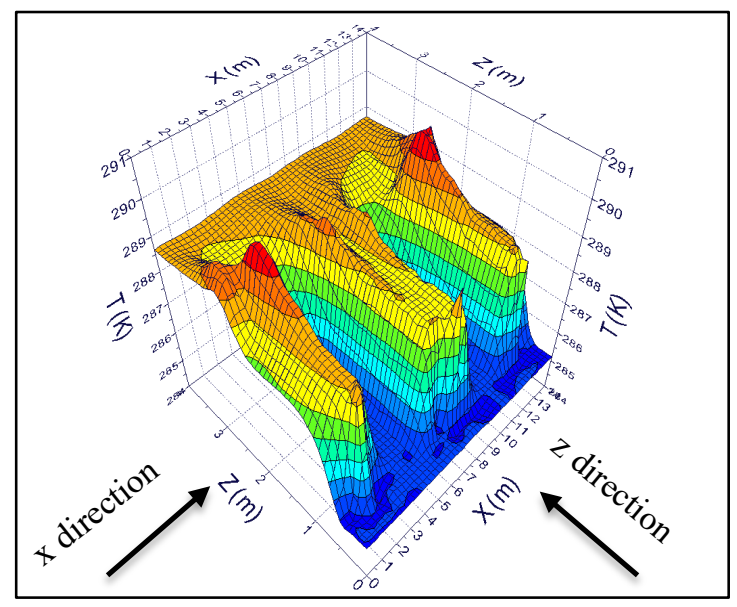

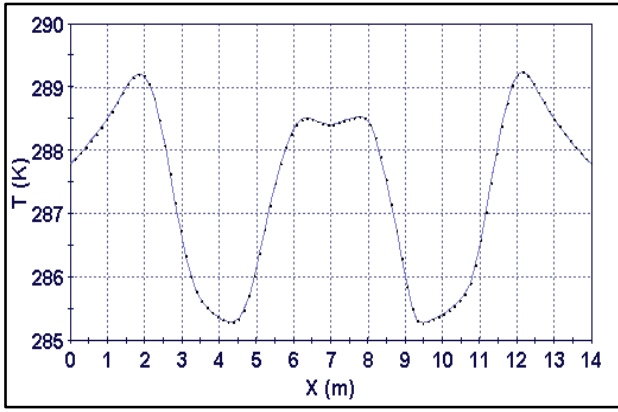

b) Temperature vs. $x$ location

Fig. IV.9.RSM visualization for T vs. $x z$ coordinates for only $20 \%$ training points

\section{b. Response surface exploration for $\mathrm{T}$ vs. $\mathrm{ZX}$ coordinates}

Fig. IV.9c shows the topology of the constructed response surface in 3D. Due to space limitation, only the $20 \%$ case is presented here. The figure depicts the nature of hot and cold aisles with hot aisles associated with high temperature, therefore, having higher elevation in the y direction. The cold aisles, and especially, the cold underfloor plenum have a lower elevation associated with low temperature in the y direction. Fig. IV.9a shows the air stratification inside the data room, and it is the projection of the response surface in the $\mathrm{x}$ direction onto the yz plane (as shown by the arrow). At about half a meter, the 
temperature is approximately $285 \mathrm{~K}\left(11.8^{\circ} \mathrm{C}\right)$. This is reasonable since the raised floor height is exactly $2 \mathrm{ft} .(0.61 \mathrm{~m})$. Fig. IV. $9 \mathrm{~b}$ is the projection of the response surface in the $\mathrm{z}$ direction onto the xy plane (as shown by the arrow). It clearly demonstrates the temperature variation in the data center model with three hot and two cold zones.

\section{c. Thermal profile reconstruction}

Now that the response surface has been formulated, we will see how close the predicted model compared to the original CFD model. By inputing all the CFD points coordinates and using the constructed response surface function, the corresponding predicted temperature for theses points are calculated. Fig. IV.10 shows the contour plot of temperature profile at the middle plane of the zx plane of the data center model (refer to

Fig. II.1). Three scenarios of different training points utilization are compared with the original CFD data points. Apparently, the higher the training points used for constructing the response surface, the more accurate the predicted model is. In the case of $20 \%$ training points utilization, the temperature profile almost resembles the orgianl CFD model with minor difference in some hotspot areas. The case of $5 \%$ training points utilization, though with many wiggling lines especially in the cold spots areas, still captures most of the aspects of the CFD model. 

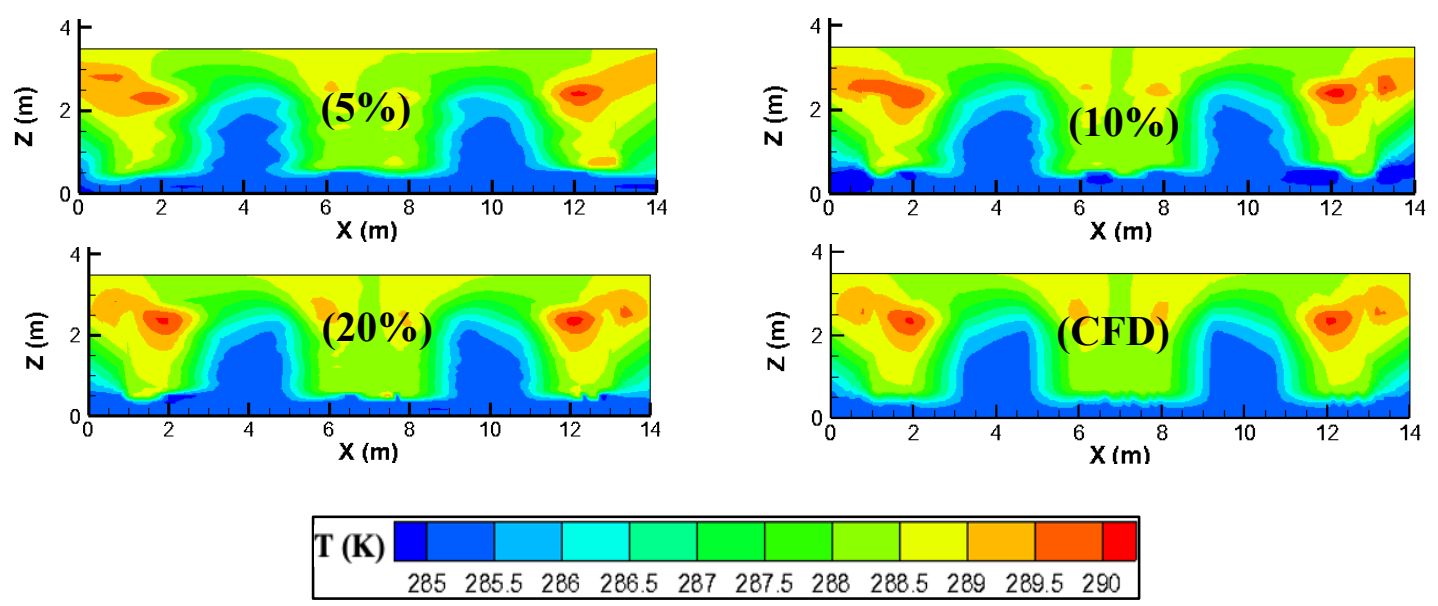

Fig. IV.10. Temperature comparison between response surface case and CFD.

\section{d. Error assessment}

In this section, we quantify how much error associated with each predicted scenario. Table IV.4 shows the error assessment for three different scenarios of training point utilization. Root mean square error calculation by eq. (IV.14) between the predicted RSM and the CFD data is calculated for comparison.

$$
R M S E=\sqrt{\frac{\sum_{i=1}^{N}\left(\hat{y}_{i}-y_{i}\right)^{2}}{n}}
$$

where $\hat{y}_{i}$ is the predicted values by RSM and $y_{i}$ is the CFD values, $n$ represents total data points.

It is shown that as the number of training points increases, the accuracy is also increasing. The max absolute error and the mean absolute error are presented for a local reference of the error, while overall RMSE shows the global assessment of the error. Though case 20 
\% shows a slightly higher in max absolute error, it has lowest RMSE and mean absolute error between the predicted RSM values and CFD data as compared to other cases.

Table IV.4. Error assessment between RSM and CFD data

\begin{tabular}{|c|c|c|c|}
\hline Cases & RMSE (K) & Max Absolute & Mean Absolute \\
& & Error (K) & Error (K) \\
\hline 5\% of original data points & 0.331 & 3.19 & 0.162 \\
\hline 10\% of original data points & 0.334 & 3.81 & 0.111 \\
\hline 20\% of original data points & 0.280 & 4.18 & 0.0607 \\
\hline
\end{tabular}

\subsubsection{Application 2: Data Center Input Design Parameter Optimization}

Normally, in order to prepare design space for optimization process requires thousands of full HT/CFD simulations, which is prohibitive and impractical to implement in reality. In this section, we demonstrate how a CFD-based RSM can be used in facilitating the optimization process of multiple design input of a data center model.

\section{a. RBF-based response surface training}

Similar to the first application, the selection of five different RBF models (eq. (IV.4) (IV.7)) was thoroughly inspected through a modeFrontier [25]. The aim is to search for a suitable RBF model that will serve as a basis function for the response surface training process. The reliability of the RBF model and the quality of the response surface 
interpolation depend upon the accuracy obtained through assessment criteria stated in eq. (IV.8) - (IV.10). 
First of all, response surface building requires a sufficient number of training points; naturally, the higher the number of training points, the more reliable the response surface. A basic rule for finding the order of magnitude of the necessary training points consists in identifying the minimum number of training points which would be necessary to compute a second order polynomial response surface and considering a number of training points which is about twice that minimum number or at least greater than that minimum. In order to obtain a good response surface, it is also important that the input parameters space is uniformly explored in the range of variations of the input parameters. The minimum number of training points for a second order polynomial response surface considering 3 input variables (i.e. mass flow inlet, inlet temperature, and server heat load):

$$
\frac{(3+2) !}{3 ! 2 !}=10
$$

Therefore, the number of training points should be about 20 .

Table IV. 5 shows the total available designs obtained from CFD simulations. These designs will be used to train the response surface.

As seen in Table IV.6, the Gaussians RBF (eq. (IV.6)) consistently dominates other RBF models in the response surface training for the $\Delta T$ output. It successfully passes the performance criteria with high fidelity in all categories. As stated before, the two most important performance assessment criteria are the $R^{2}$ and the $R A A E$. As seen this table, the Gaussian RBF provides the largest $R^{2}$, as well as the smallest $R A A E$, which gives a confidence for the construction of the response surface. Furthermore, it is also the case that has the smallest $R M A E$, meaning the consistency is uniformly kept throughout the computational domain. 
Table IV.5. CFD-simulated real designs for the RSM training

\begin{tabular}{|c|c|c|c|c|c|c|}
\hline Cases & $\mathbf{T}_{\text {in }}\left({ }^{\circ} \mathbf{C}\right)$ & $\mathbf{m}_{\text {flow_in }}(\mathbf{k g} / \mathbf{s})$ & $Q_{1}(W)$ & $\operatorname{Tmin}\left({ }^{\circ} \mathbf{C}\right)$ & $\operatorname{Tmax}\left({ }^{\circ} \mathrm{C}\right)$ & $\Delta \mathbf{T}\left({ }^{\circ} \mathbf{C}\right)$ \\
\hline 1 & 11.8 & 5.5 & 900 & 11.95 & 21.959 & 10.009 \\
\hline 2 & 12.8 & 5.5 & 900 & 12.95 & 22.916 & 9.966 \\
\hline 3 & 13.8 & 5.5 & 900 & 13.95 & 23.916 & 9.966 \\
\hline 4 & 14.8 & 5.5 & 900 & 14.95 & 24.959 & 10.009 \\
\hline 5 & 15.8 & 5.5 & 900 & 15.95 & 25.916 & 9.966 \\
\hline 6 & 11.8 & 5.0 & 900 & 15.95 & 25.916 & 9.966 \\
\hline 7 & 12.8 & 6.0 & 900 & 12.95 & 22.475 & 9.525 \\
\hline 8 & 13.8 & 7.0 & 900 & 13.95 & 22.515 & 8.565 \\
\hline 9 & 14.8 & 8.0 & 900 & 14.95 & 23.377 & 8.427 \\
\hline 10 & 15.8 & 9.0 & 900 & 15.95 & 25.152 & 9.202 \\
\hline 11 & 12.8 & 5.3 & 900 & 12.95 & 23.133 & 10.183 \\
\hline 12 & 12.8 & 5.8 & 900 & 12.95 & 22.611 & 9.661 \\
\hline 13 & 12.8 & 6.3 & 900 & 12.95 & 22.174 & 9.224 \\
\hline 14 & 12.8 & 6.8 & 900 & 12.95 & 21.607 & 8.657 \\
\hline 15 & 12.8 & 7.3 & 900 & 12.95 & 21.862 & 8.912 \\
\hline 16 & 12.8 & 5.6 & 1000 & 12.95 & 23.929 & 10.979 \\
\hline 17 & 12.8 & 6.1 & 1500 & 12.95 & 28.653 & 15.703 \\
\hline 18 & 12.8 & 6.6 & 2000 & 12.95 & 32.631 & 19.681 \\
\hline 19 & 12.8 & 7.1 & 2500 & 12.95 & 38.042 & 25.092 \\
\hline 20 & 12.8 & 7.6 & 3000 & 12.95 & 42.457 & 29.507 \\
\hline
\end{tabular}

Table IV.6. Performance measurement among different RBF models for $\Delta \mathrm{T}$ output

\begin{tabular}{|c|c|c|c|}
\hline & R square & RAAE & RMAE \\
\hline Multiquadrics & 0.9980 & 0.0055 & 0.0368 \\
\hline Inverse Multiquadrics & 0.9981 & 0.0053 & 0.0379 \\
\hline Gaussians & 0.9984 & 0.0048 & 0.0321 \\
\hline Polyharmonic Splines & 0.9978 & 0.0055 & 0.0373 \\
\hline Wendland's form & 0.9980 & 0.0052 & 0.0369 \\
\hline
\end{tabular}




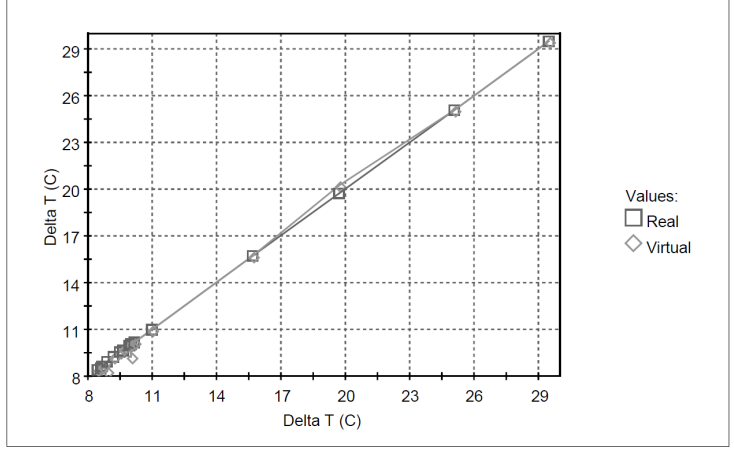

a) Multiquadrics

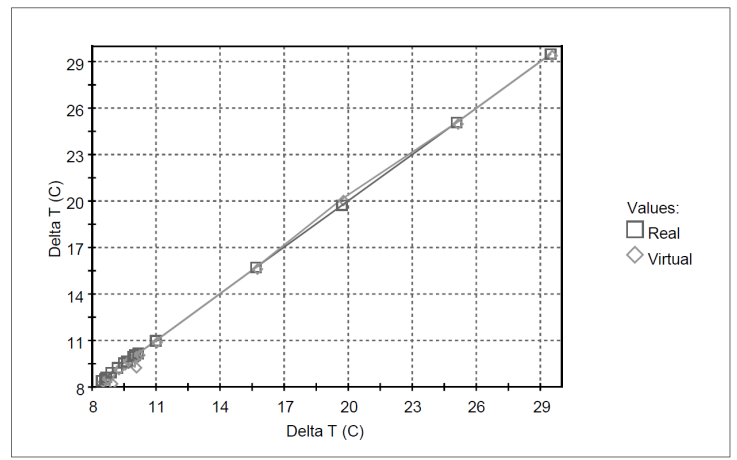

c) Gaussians

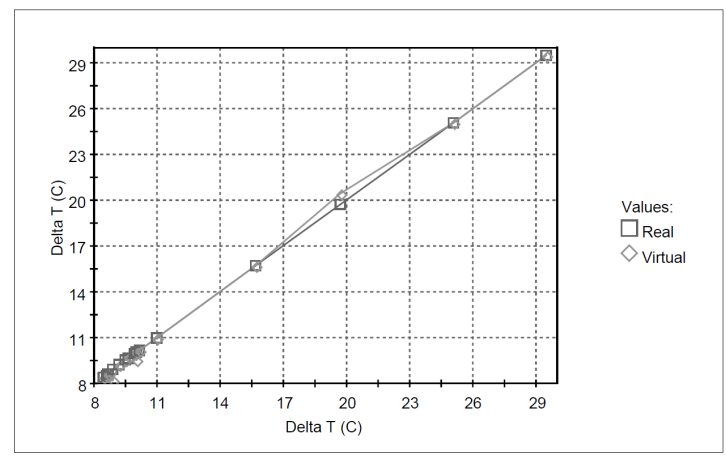

e) Wendland's form

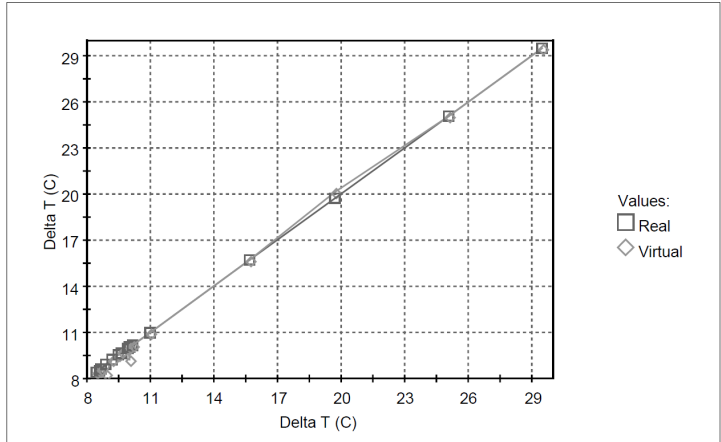

b) Inverse Multiquadrics

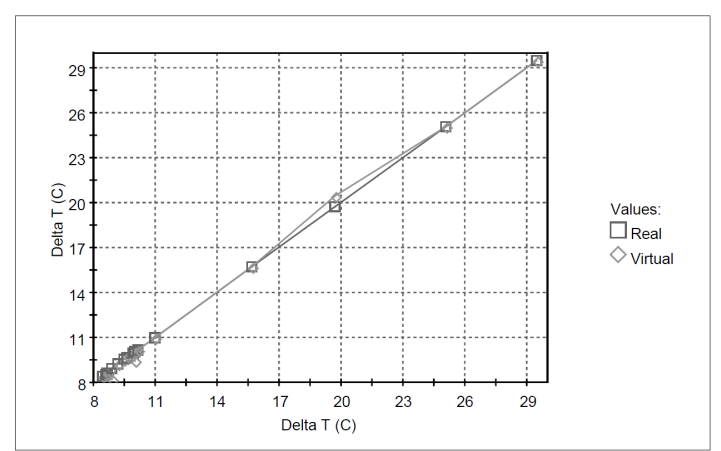

d) Polyharmonic Splines

Fig. IV.11. Distance chart comparison among 5 different RBF models

Fig. IV.11 shows the deflection comparison of the $\Delta \mathrm{T}$ output for five different RBF models. Ideally, if a response surface is able to identify the behavior of the system, the $\Delta \mathrm{T}$ outputs computed by the response surface (virtual designs) will coincide with the ones obtained from CFD-based data (real designs). Therefore, a diagonal line of approximately $45^{\circ}$ is plotted. In this figure, real and virtual points are plotted together, so the deflection can be visualized and compared among different RBF models. The stronger the deflection is, the 
unlikely the RBF model will be selected as the basis of the response surface construction. In general, five RBF models show indistinctive differences and are equally good. However, the Gaussians model will be picked according to its best performance proven in Table IV.6.

\section{b. Response surface exploration for $\Delta T$ vs. design input}

After selecting the proper RBF model for the response surface training, we can generate the hypersurface using the training points. As a rule of thumb, the more training points the more accurate the response surface is. However, in real life situation, sometimes it is very expensive to achieve many data points due to serious simulation time. As mentioned before, the minimum number of training points for three design parameters, i.e. 20 points, were utilized to construct the response surface.

In order to obtain a good response surface, it is also important that the input parameters space is uniformly explored in the range of variations of the input parameters. Here, the input parameters are the mass flow inlet $(5.3-9.0 \mathrm{~kg} / \mathrm{s})$, the inlet temperature $\left(11.8^{\circ} \mathrm{C}-\right.$ $\left.15.8^{\circ} \mathrm{C}\right)$, and the server head load $(900-3000 \mathrm{~W})$, while the output parameter is the temperature.

Fig. IV.12 shows the topology of the constructed response surface in 3D looking at three different combinations of the design parameter inputs. All charts are plotted based upon the $\Delta T$ between the maximum and the minimum temperatures inside the data center model

against the design parameters, i.e., supply air mass flowrate, supply air temperature, and 
server heat load. These charts give a good visualization of the hypersurface constructed in space, and they all refer to only one response surface. The trained response surface is then used in place of an actual HT/CFD simulation during the optimization process for a much faster calculation.

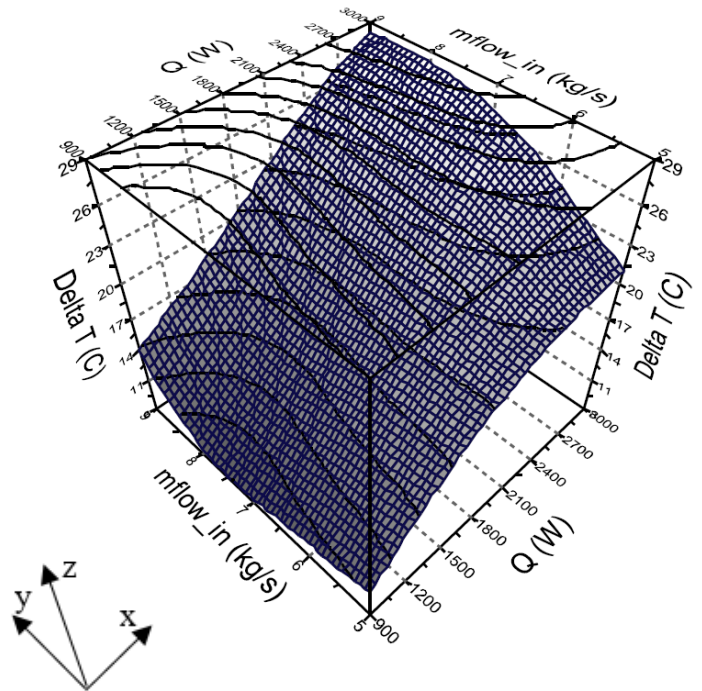

a) Perspective 1

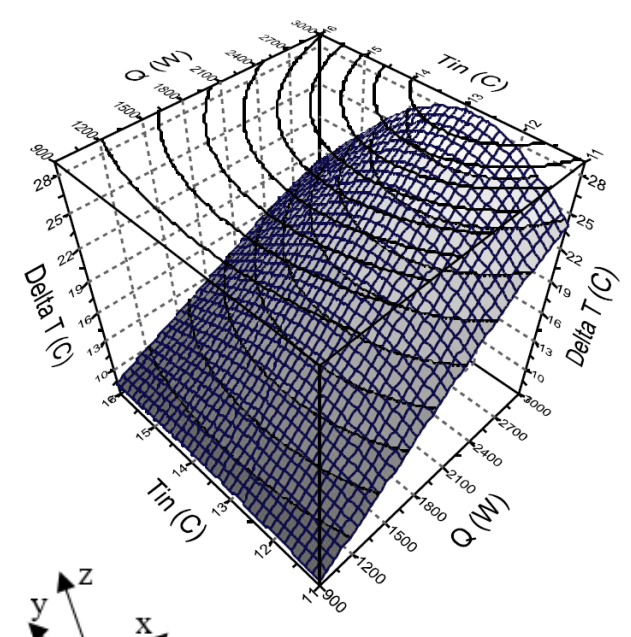

b) Perspective 2

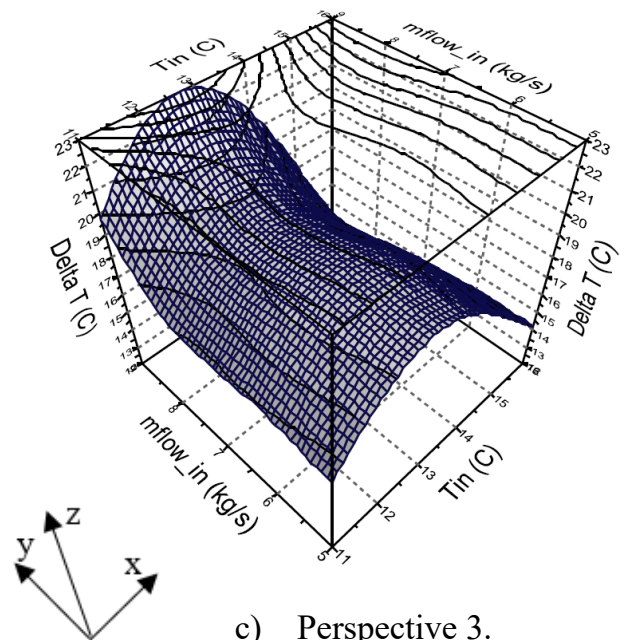

Fig. IV.12. RSM visualization for $\Delta T$ versus different design parameters inputs 
The effect of each design parameter on the $\Delta T$ across the server racks is shown in Fig. IV.13. Ideally, our goal is to keep a lower $\Delta T$ within the data center, which indicates that a more uniform air surrounding the server racks is present and that the server racks will not suffer a hot spot problem. In Fig. IV.13a, though showing some fluctuations at lower inlet mass flow rates, the $\Delta T$ is kept below $19.2^{\circ} \mathrm{C}$. As the mass flow rate goes beyond $8.3 \mathrm{~kg} / \mathrm{s}$ the $\Delta T$ increases exponentially.

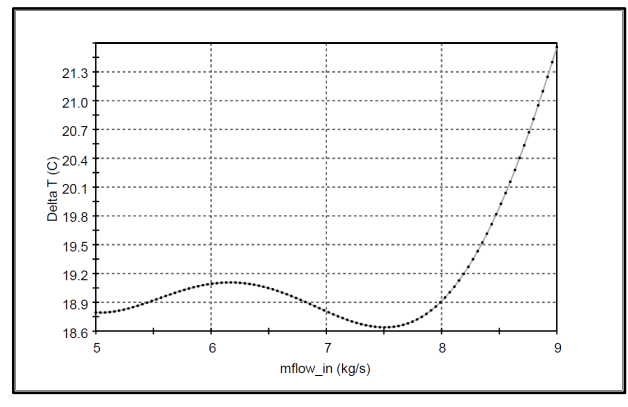

a) $\Delta T$ vs. mass flow inlet

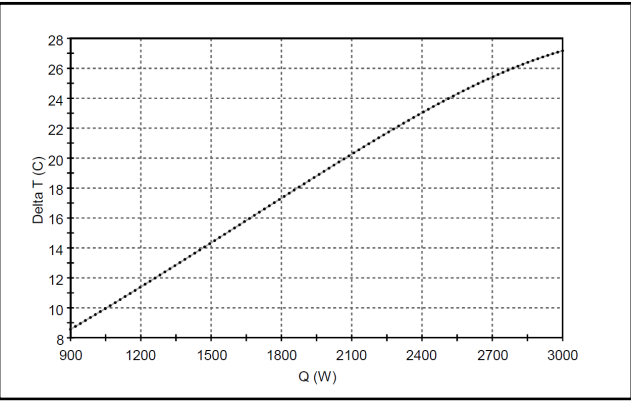

b) $\Delta T$ vs. server heat load

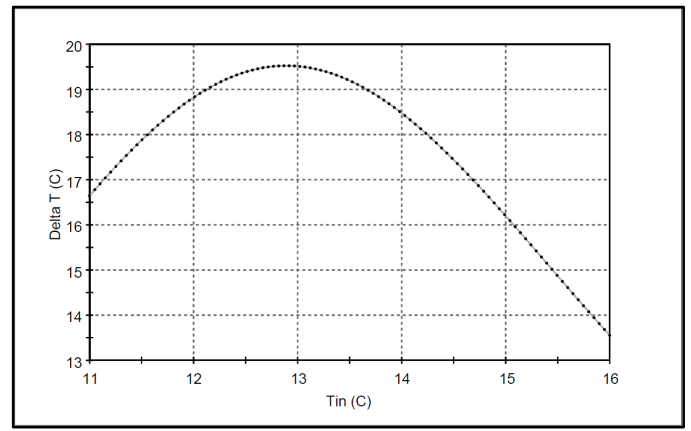

c) $\Delta T$ vs. inlet temperature

Fig. IV.13. The effect of each design parameter on the $\Delta T$

Next, we look at the effect of server heat load on the $\Delta T$ as seen in Fig. IV.13b. Generally, as the server heat load is increased, the temperature inside the data center will also increase. The relationship is quite linear. For each $100 \mathrm{~W}$ increase in server heat load, the $\Delta \mathrm{T}$ will 
increase by $1^{\circ} \mathrm{C}$. In Fig. IV.13c, the effect of inlet temperature is investigated. Within the inlet temperature range investigated, it shows that a peak in $\Delta T$ is spotted at about $13^{\circ} \mathrm{C}$. If we go lower than this temperature point, we will have a smaller $\Delta \mathrm{T}$, but then we will consume more energy to achieve this cooler temperature. On the other hand, if we go higher $13^{\circ} \mathrm{C}$, we will also get a lower $\Delta \mathrm{T}$, which is good for the current configuration in terms of both energy efficiency and safety for the servers.

We have looked at each individual effect of the design parameters on the $\Delta \mathrm{T}$. In the next section, we will see how three of the design parameters act together to affect the $\Delta \mathrm{T}$ as well as the maximum temperature outcomes. The goal is to minimize both of these outputs. In this case, we are forming a two-objective optimization. The optimization process is carried out to search for a set of points in the objective space that dominate all other points. These points are non-dominant with respect to each other and form a hyper-surface in the objective space, called Pareto front.

\section{c. RSM-based Multi-objective optimization}

Multi-objective genetic algorithm (MOGA) was used for a fast Pareto convergence. It is imperative to use the response surface metamodel to create the generations for the MOGA scheme since real simulated designs are difficult to achieve due to a large number of generations must be created and their huge simulation time required. ModeFrontier proram was used to carry out the study. 


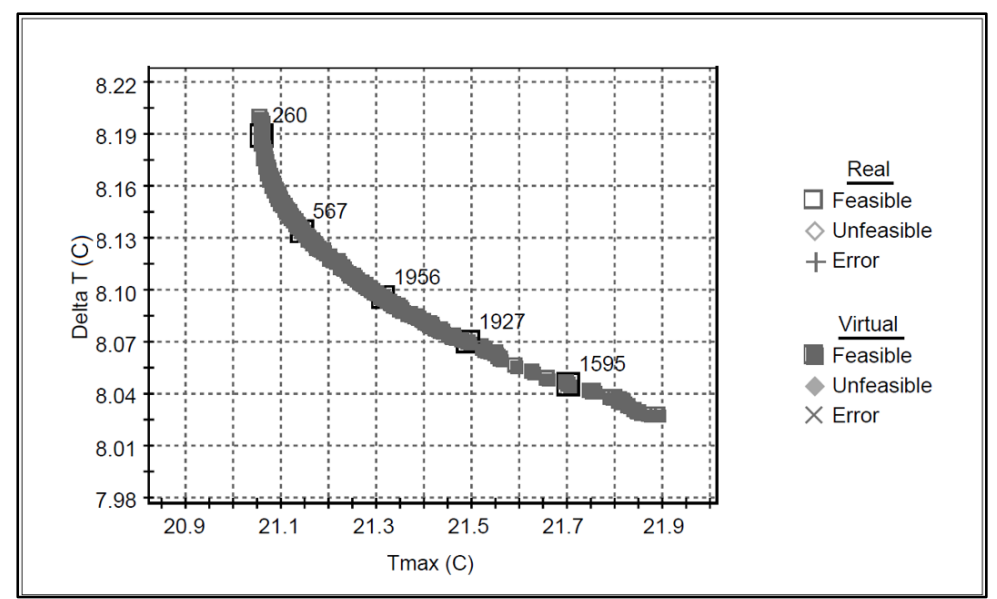

Fig. IV.14. Pareto designs

Fig. IV.14 shows the Pareto design of the best tradeoffs between the $\Delta \mathrm{T}$ and the maximum temperature within the data center model. These Pareto designs form a border line called Pareto front. Points lied within this front represent an equally good design toward the two objectives studied, i.e., $\Delta \mathrm{T}$ and the maximum temperature. It is found that the inlet temperature and the inlet mass flow rate ranges for these optimal designs are from $11.8-$ $12.62^{\circ} \mathrm{C}, 7.36-7.67 \mathrm{~kg} / \mathrm{s}$, respectively. Also, all of the Pareto designs have a server heat load of $900 \mathrm{~W}$. In order to test the confidence of the Pareto designs, let us randomly select five designs in the Pareto Front and run the CFD simulations for these designs and not using the response surface. We will plot them together with the Pareto designs to see if they still lie on the Pareto Front. The locations of the five randomly selected Pareto designs are also shown in Fig. IV.14. 


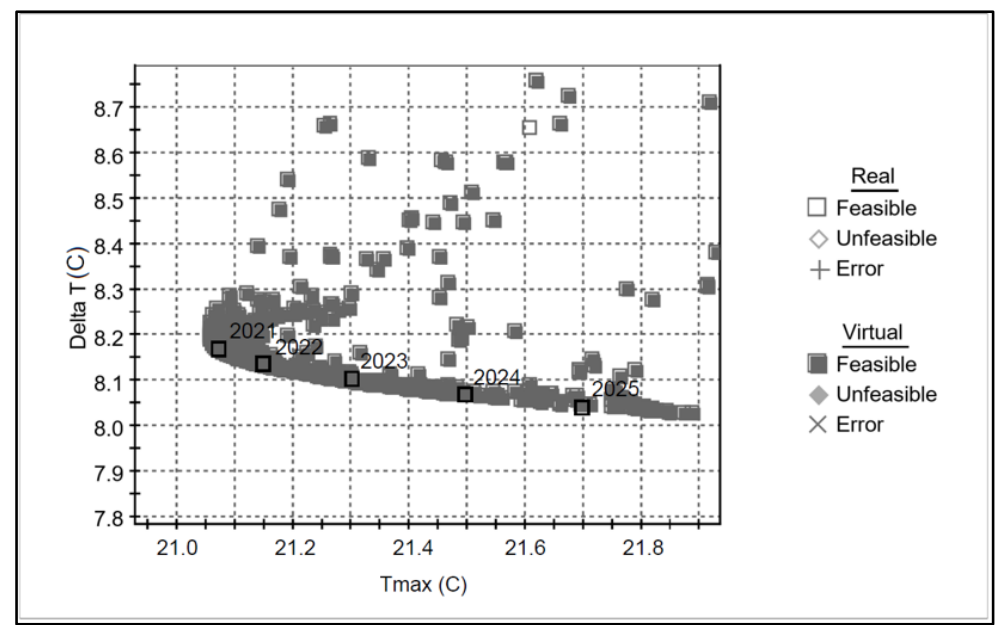

Fig. IV.15. Validation for the five selected Pareto designs

After running the five selected designs using the CFD simulations, we plotted them along with all the generated designs from the MOGA run. As expected, the positions of the new designs are almost the same as identified by the response surface, meaning that they represent the three solutions on the real Pareto Front. (see Fig. IV.15).

\subsubsection{Application 3: Floor Tile Airflow Uniformity Optimization}

In this section, the objective is to use optimization based on RSM to seek the best distribution in tile porosities that can provide a uniform airflow distribution for all tiles. For achieving this goal, response surfaces associated with four different RBF models (eqs. (IV.4) - (IV.7)) were carefully trained and evaluated. The aim was to search for a suitable RBF corresponding to a response surface training. The reliability of the RBF model and the accuracy of the response surface interpolation depend upon the accuracy obtained through assessment criteria stated in eqs. (IV.8) - (IV.10). Each response surface 
construction requires a sufficient number of training points. Naturally, the higher the number of training points, the more reliable the response surface. A basic rule for finding the order of magnitude of the necessary training points consists in identifying the minimum number of training points which would be necessary to compute a second order polynomial response surface and considering a number of training points which is about twice that minimum number or at least greater than that minimum. The minimum number of training points to ensure the input parameter space is uniformly explored in the range of variations of the input parameters is shown in eq. (IV.15). [25]

$$
P \geq \frac{(L+k) !}{L ! k !}
$$

where $P$ is the number of training points, $L$ is number of input variables, $k$ is the degree order of polynomial. Therefore, with eight tile porosities input, the minimum number of training point for a second order polynomial response surface is 45 design cases. Due to space limit, only 10 out of 45 designs are shown in Table IV.7. In order to obtain a good response surface, it is also important that the input parameters space is uniformly explored in the range of variations of the input parameters. In the range of exploration from $10 \%$ to $80 \%$ tile porosity, Sobol's algorithm [66] was used for the preparation of training design cases. The algorithm provides a random selection of porosity arrangement uniformly distributed in the proposed range of exploration.

After performing the response surface training for four different RBF models, the next step is to assess the credibility of each model. Ideally, if a response surface is able to identify the behavior of the system, the output (standard deviation of the tile mass flow rate) computed by the response surface will coincide with the CFD-simulated values. In Fig. 
IV.16, real and virtual points represent CFD-simulated values and the RSM-predicted values, respectively. Both multiquadrics and inverse multiquadrics RBFs do a good job in predicting the designs. However, out of four RBF models investigated, the values predicted by multiquadrics RBF are the closest to the simulated values with smallest difference, while the values predicted by Gaussians RBF are the worst in terms of fluctuating distance.

Table IV.7. Initial designs for response surface construction

\begin{tabular}{|c|c|c|c|c|c|c|c|c|}
\hline Cases & \multicolumn{9}{|c|}{ Tile Porosity } \\
\hline & Tile 1 & Tile 2 & Tile 3 & Tile 4 & Tile 5 & Tile 6 & Tile 7 & Tile 8 \\
\hline $\mathbf{1}$ & 0.28 & 0.63 & 0.63 & 0.63 & 0.28 & 0.28 & 0.63 & 0.28 \\
\hline $\mathbf{2}$ & 0.63 & 0.28 & 0.28 & 0.28 & 0.63 & 0.63 & 0.28 & 0.63 \\
\hline $\mathbf{3}$ & 0.71 & 0.71 & 0.19 & 0.36 & 0.71 & 0.54 & 0.71 & 0.36 \\
\hline $\mathbf{4}$ & 0.36 & 0.36 & 0.54 & 0.71 & 0.36 & 0.19 & 0.36 & 0.71 \\
\hline $\mathbf{5}$ & 0.19 & 0.54 & 0.36 & 0.19 & 0.19 & 0.36 & 0.54 & 0.54 \\
\hline $\mathbf{6}$ & 0.54 & 0.19 & 0.71 & 0.54 & 0.54 & 0.71 & 0.19 & 0.19 \\
\hline $\mathbf{7}$ & 0.58 & 0.67 & 0.41 & 0.76 & 0.14 & 0.67 & 0.76 & 0.41 \\
\hline $\mathbf{8}$ & 0.23 & 0.32 & 0.76 & 0.41 & 0.49 & 0.32 & 0.41 & 0.76 \\
\hline $\mathbf{9}$ & 0.41 & 0.49 & 0.23 & 0.58 & 0.67 & 0.14 & 0.58 & 0.58 \\
\hline $\mathbf{1 0}$ & 0.76 & 0.14 & 0.58 & 0.23 & 0.32 & 0.49 & 0.23 & 0.23 \\
\hline
\end{tabular}

For an assurance of the best RBF model, performance criteria recommended in eq. (IV.8) - (IV.10) were evaluated. The iterative stages were executed to seek a converged result (optimum design), and at each stage validation was performed to ensure the suitable designs are selected to proceed to the next stage. After four stages, a desirable design was achieved with no further stage required since only minimal change was occurred after the fourth stage. As seen in Table IV.8a, for the first iterative stage of the optimization process, the multiquadric RBF (eq. (IV.4)) consistently dominates other RBF models in three performance metrics in terms of $R$-squared, $R A A E$, and $R M A E$. Indeed, it has the highest 
$R$-squared measurement, lowest $R A A E$ and $R M A E$. In the next three iterative stages (Table IV. $8 \mathrm{~b}, \mathrm{c}, \& \mathrm{~d})$, it is also the multiquadrics that stands out as the most suitable RBF model for training the response surface.

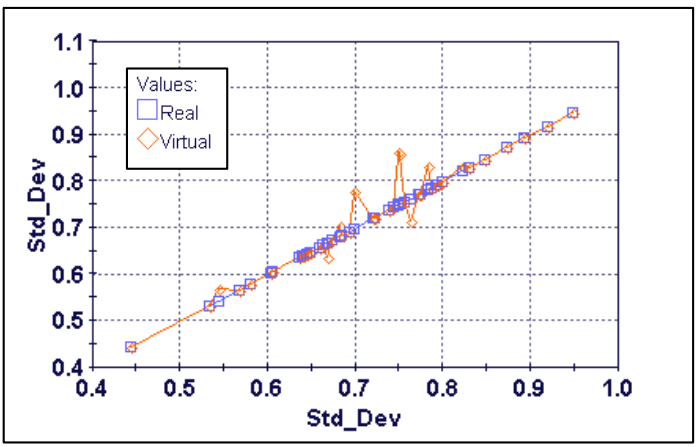

a) Multiquadrics

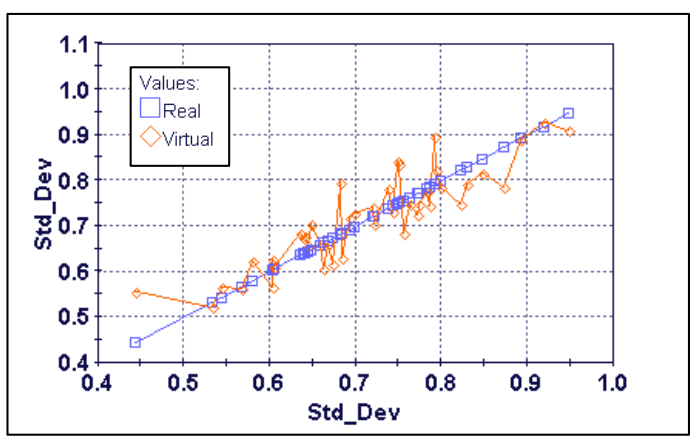

c) Gaussians

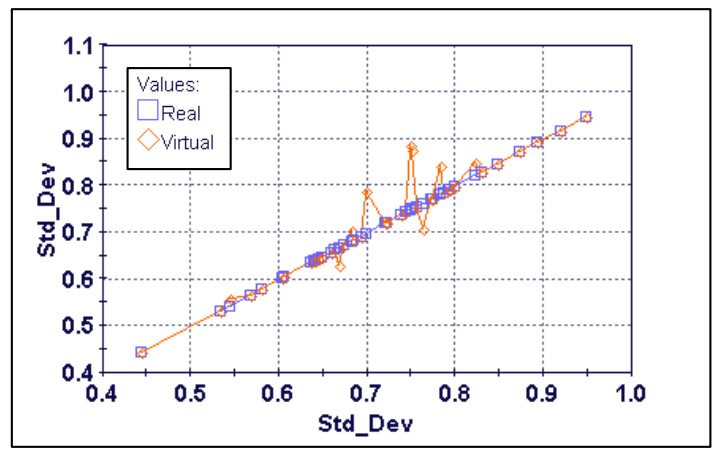

b) Inverse Multiquadrics

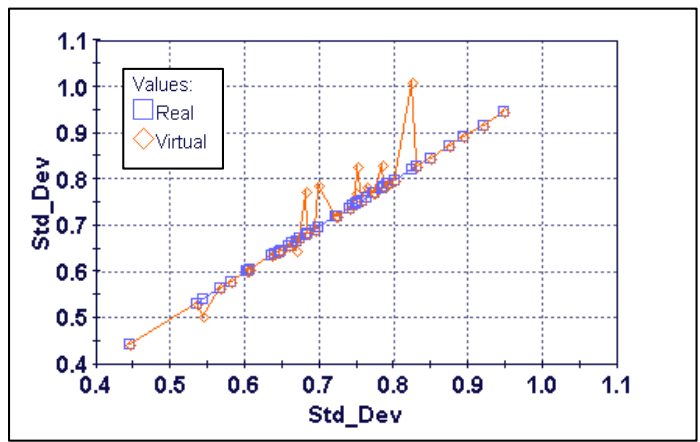

d) Polyharmonic Splines

Fig. IV.16. Distance chart between real designs by CFD and virtual designs by RSM

Now that the suitable response surface based on the multiquadrics RBF is selected, the 3$\mathrm{D}$ response surface is then explored. Because of the limit of space in this section, only the response surface constructed for the optimization in the $1^{\text {st }}$ iterative design stage is presented. There are also 8 tile porosity inputs, but only the first two tiles are presented. It should be pointed out that tiles with different porosities can be created from tiles where the 
same number, size, shape and distribution of the perforations is used. Then, a parallel thin plate with identical perforations can be moved adjacent to the perforated tile, thus, changing effective porosity of the perforated tile.

Table IV.8. RBF performance assessment for 4 different stages

\begin{tabular}{|c|c|c|c|}
\hline Functions & RAAE & RMAE & R-squared \\
\hline Multiquadrics & 0.098 & 1.018 & 0.934 \\
\hline Gaussians & 0.379 & 1.148 & 0.789 \\
\hline Inverse Multiquadrics & 0.111 & 1.215 & 0.915 \\
\hline Polyharmonic Splines & 0.114 & 1.589 & 0.909 \\
\hline
\end{tabular}

\begin{tabular}{|c|c|c|c|}
\hline Functions & RAAE & RMAE & R-squared \\
\hline Multiquadrics & 0.061 & 0.706 & 0.977 \\
\hline Gaussians & 0.108 & 1.444 & 0.913 \\
\hline Inverse Multiquadrics & 0.067 & 0.825 & 0.971 \\
\hline Polyharmonic Splines & 0.109 & 1.842 & 0.901 \\
\hline
\end{tabular}

a) Stage 1

\begin{tabular}{|c|c|c|c|}
\hline Functions & RAAE & RMAE & R-squared \\
\hline Multiquadrics & 0.034 & 0.364 & 0.994 \\
\hline Gaussians & 0.071 & 1.258 & 0.961 \\
\hline Inverse Multiquadrics & 0.036 & 0.472 & 0.993 \\
\hline Polyharmonic Splines & 0.068 & 0.954 & 0.967 \\
\hline
\end{tabular}

c) Stage 3 b) Stage 2

\begin{tabular}{|c|c|c|c|}
\hline Functions & RAAE & RMAE & R-squared \\
\hline Multiquadrics & 0.028 & 0.311 & 0.996 \\
\hline Gaussians & 0.099 & 0.921 & 0.951 \\
\hline Inverse Multiquadrics & 0.031 & 0.314 & 0.996 \\
\hline Polyharmonic Splines & 0.048 & 0.736 & 0.987 \\
\hline
\end{tabular}

d) Stage 4

The shape of the 3D response surface is shown in Fig. IV.17a, while the projections onto each plane surface are presented in Fig. IV.17a, b, \& c.

After the suitable response surface was constructed, the optimization process proceeded in searching for the best arrangement of mixed-porosity floor tiles to obtain the minimum total deviation in air flow rates. During one stage, thousands of designs with different tile porosity combinations between $10 \%-80 \%$ (labeled with $\mathrm{t} 1, \mathrm{t} 2, \ldots, \mathrm{t} 8$ ) were generated with GA based entirely on the corresponding trained response surface. These generated designs 
are all plotted in Fig. IV.18, and each chart in this figure corresponds to one stage labeled. A constraint of 0.05 is placed on the standard deviation to highlight the best population at each stage. A few best designs from this population were then selected and validated against the simulated designs. The predicted designs passing the selection test criteria discussed later in this section, were added to the original data set for the next stage of optimization, and the whole process including RSM training and generation of predicted designs by GA was repeated. It was found that after four stages of optimization, the objective for best floor arrangement was reached.

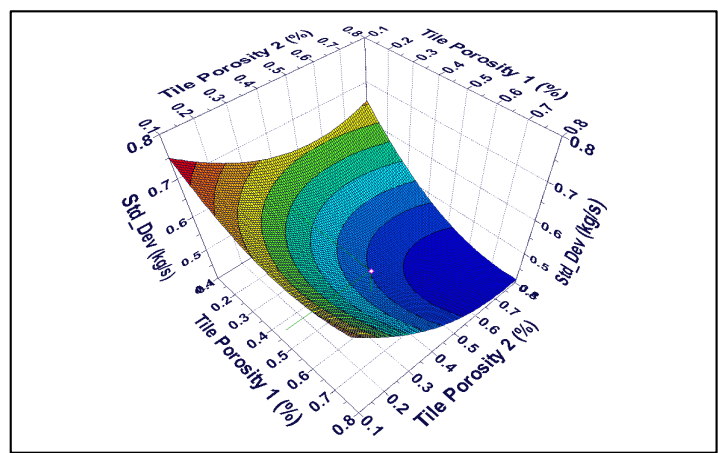

a) 3D response surface of the STD deviation

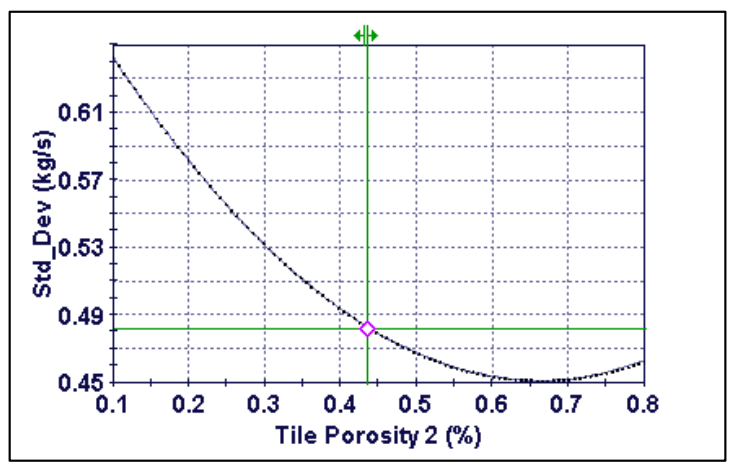

c) Standard deviation vs. tile 2 porosity

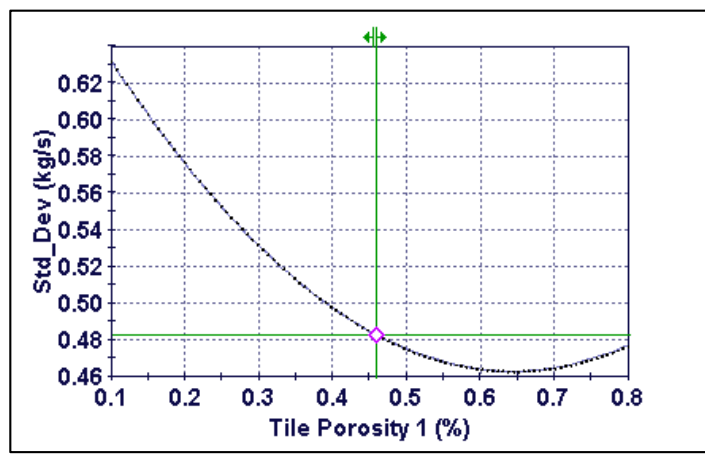

b) Standard deviation vs. tile 1 porosity

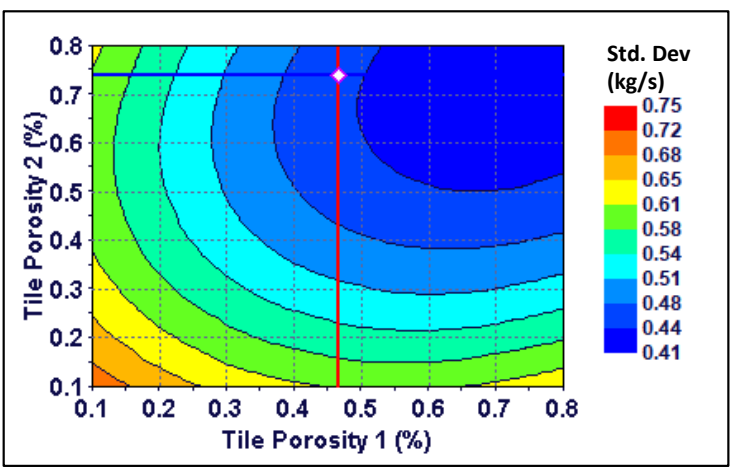

d) Tile 2 porosity vs. tile 1 porosity

Fig. IV.17. 3D response surface exploration for multiquadrics in the $1^{\text {st }}$ stage (modeFrontier) 


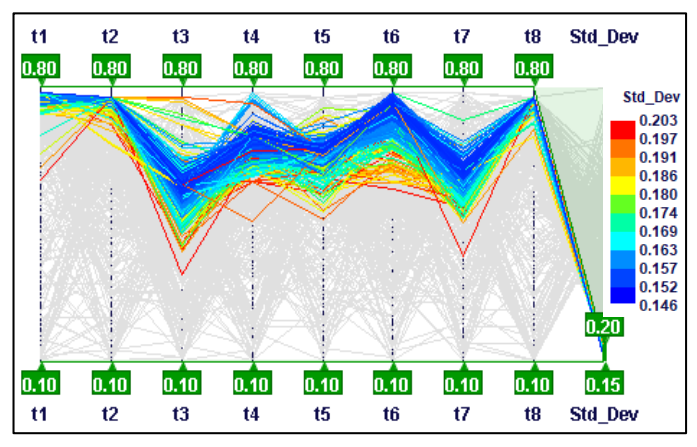

a) Stage 1

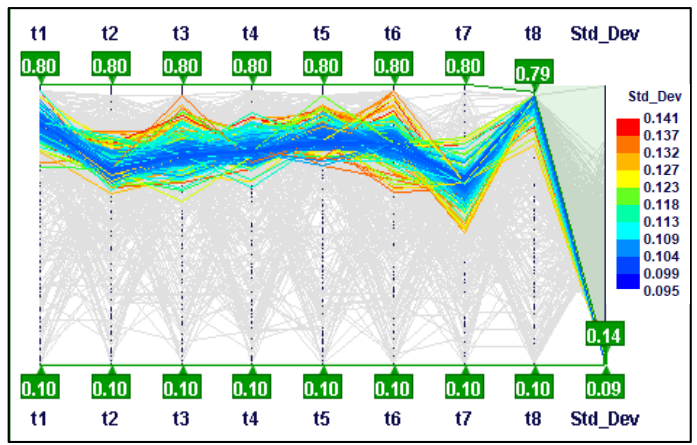

c) Stage 3

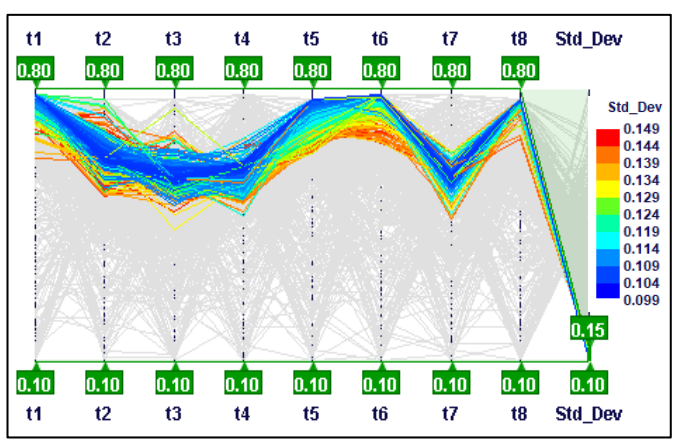

b) Stage 2

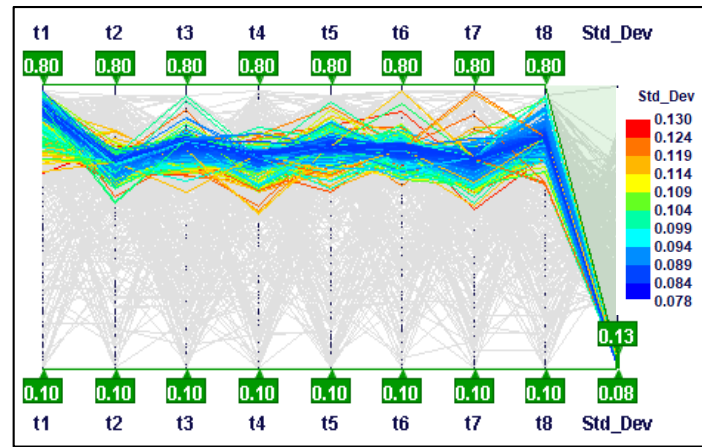

d) Stage 4

Fig. IV.18. Optimum tile porosity combinations at different stages (modeFrontier)

The selection criteria were considered with priority order given as follows: 1) the simulated design value is smaller than the predicted design value, or the difference between predicted and simulated designs is within 10 percent; 2) designs with smallest differences are acceptable. The first criterion is considered because simulated designs with smaller standard deviations help driving the process closer to the desirable solution. If the first criterion cannot be met, then the second criterion helps selecting the closest simulated designs for the next stage of optimization. At least five simulated designs corresponding to five predicted designs that are selected by the selection criteria were then added to the original dataset for the next stage of optimization including new RSM. 


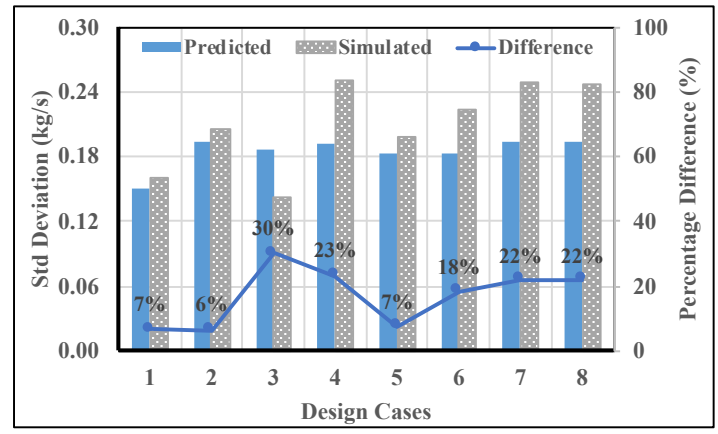

a) Stage 1

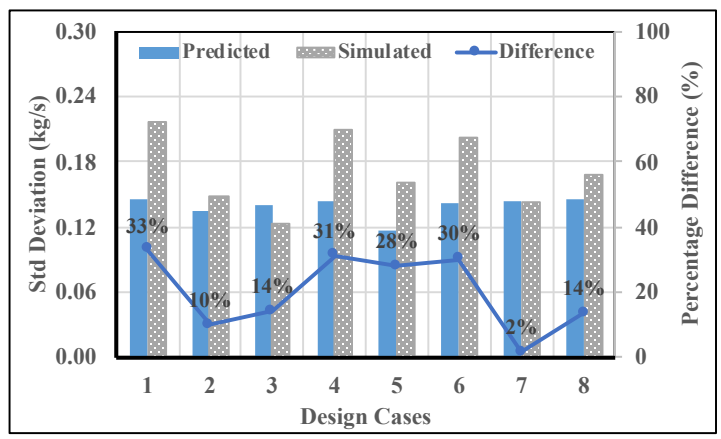

c) Stage 2

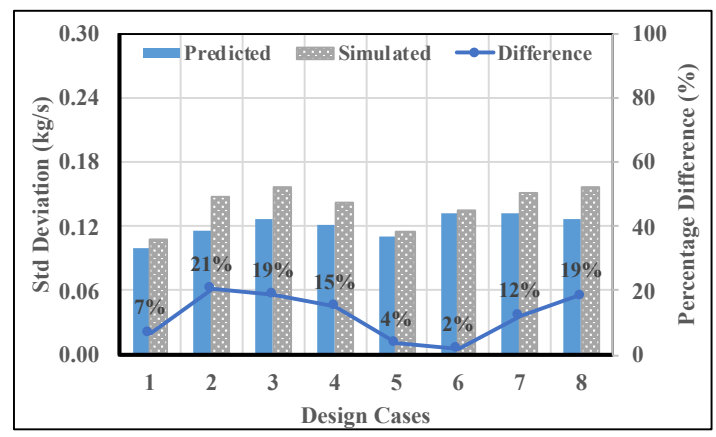

e) Stage 3

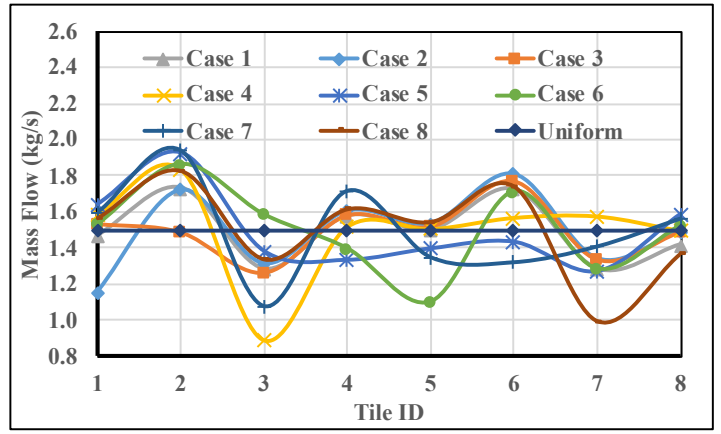

b) Stage 1

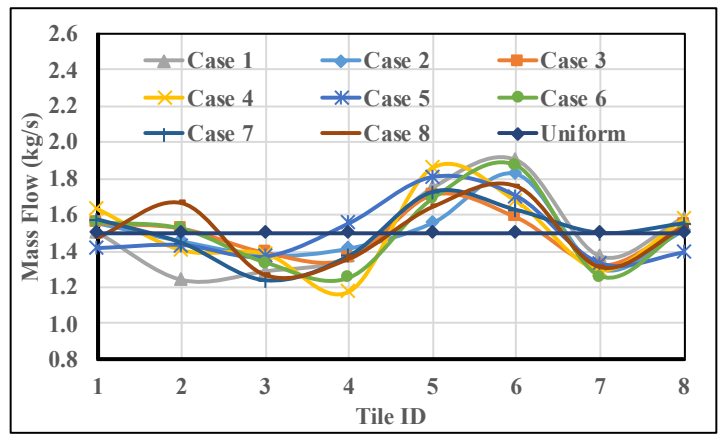

d) Stage 2

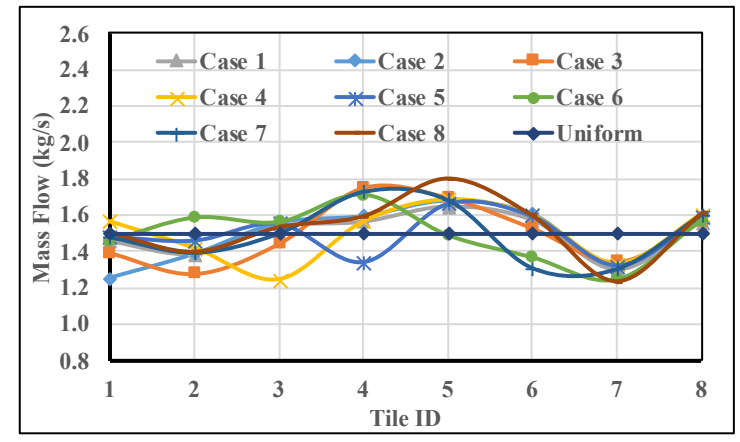

f) Stage 3

Fig. IV.19. Design validation via stages

a), c), e) Comparison of the tile mass flow rate standard deviation between the predicted RSM and the simulated CFD values. b), d), f) The simulated CFD values of tile mass flow rate for cases validated at each stage

Fig. IV.19 shows the validation process at the first three stages of the design process. This

figure shows that "difference" reduces with increasing number of "stages", which suggests 
that the entire design process is converging. Fig. IV.19b, d, and f also shows the mass flow rates for all tiles slowly become flatter throughout the stages. This serves as a basis for the success of the fourth stage when the final design outperforms the original design in standard deviation as shown in Fig. IV.2la. Although there are eight design cases are validated as each stage, only five good ones are selected to carry on the next optimization stage.

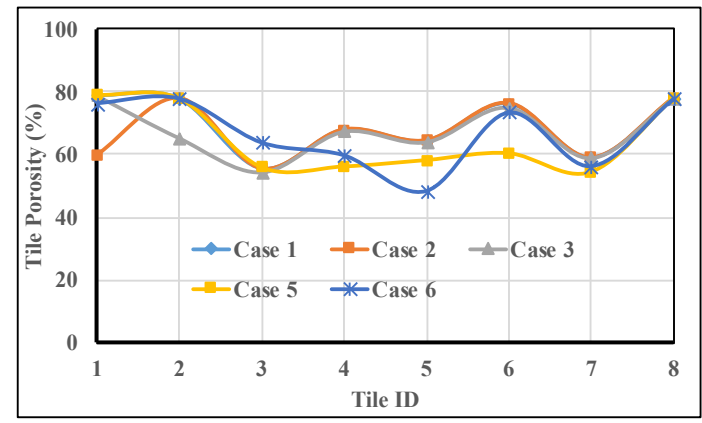

a) Stage 1

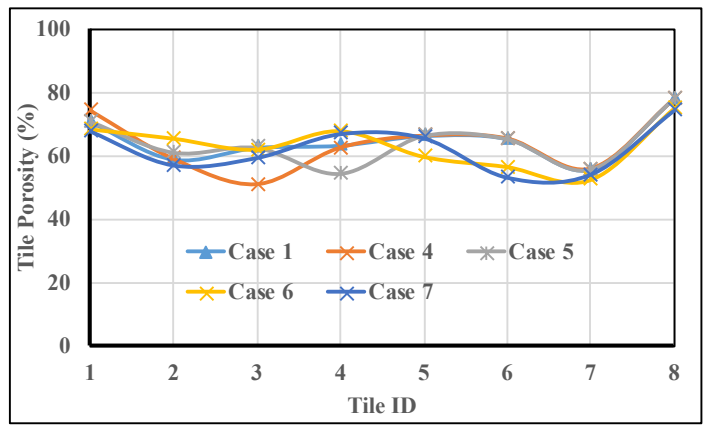

c) Stage 3

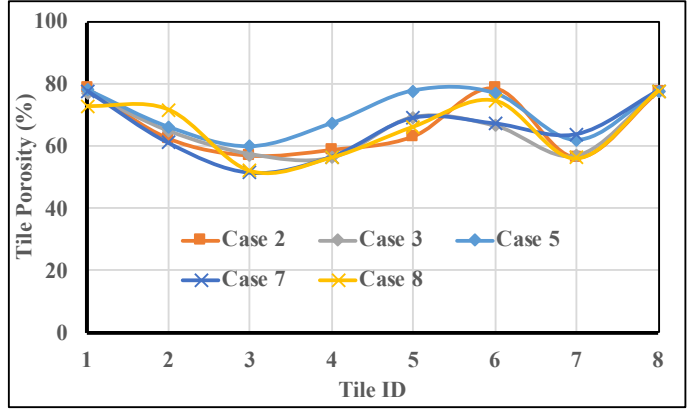

b) Stage 2

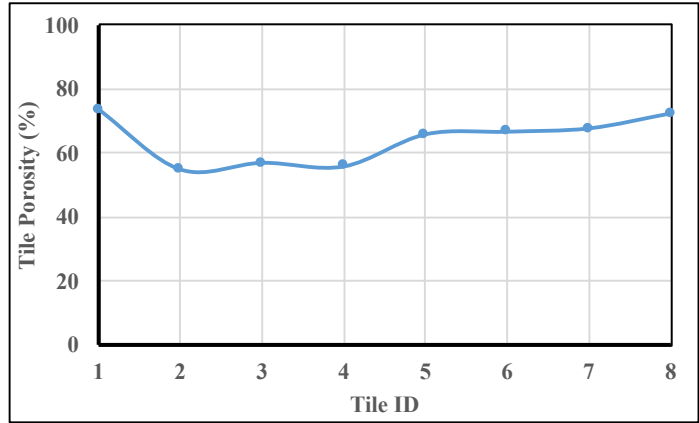

d) Stage 4

Fig. IV.20. Tile porosity for best-validated designs at each stage

The tile porosity configuration of design cases selected at each stage are shown in Fig. IV.20. From the mentioned selection criteria, there are five validated designs are selected in the first three stages. The predicted design shown in stage 4 is the final product of the 
optimization process. The tile porosity arrangement of the final design is varied from $50 \%$ $-70 \%$. The final predicted design was also validated and reflected in the simulated design. This design is improved over the original design with all uniform tiles at $56 \%$ porosity. (see Fig. IV.21).

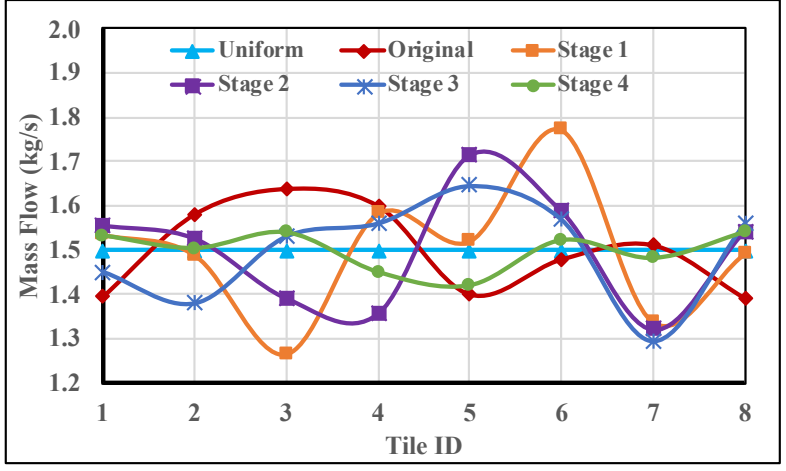

a) Tile mass flow rate for all best designs at each stage compared to the original and uniform cases

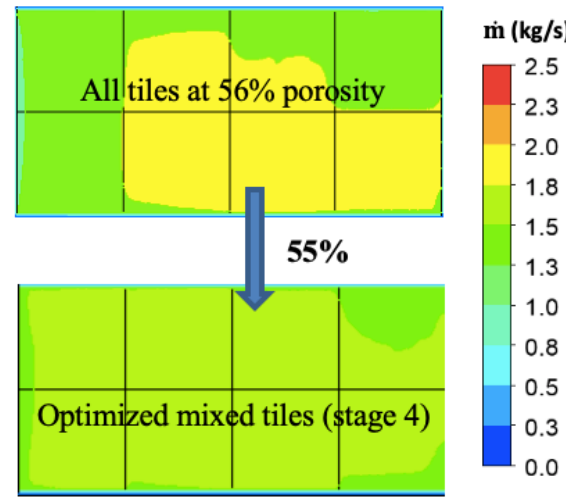

b) Relative tile airflow uniformity improvement

Fig. IV.21. Tile mass flow rate comparison

The tile mass flow rate evolution throughout iterative stages of the optimization process is demonstrated in Fig. IV.2la. Indeed, improvement through stages with respect to the airflow uniform distribution line is observed. Also, the contour plot of the final best design compared with the original design is shown in Fig. IV.2lb. The final design in stage 4 almost reaches the uniformity with a relative improvement in standard deviation of 55\% compared to the original design with all tiles at $56 \%$ porosity. 


\subsubsection{Application 4: Inverse Design for Floor Tile Optimization}

Sometimes, due to the uneven thermal load distribution of server racks within a data center, the demand for supply cool air from perforated tiles are also varied in the cold aisle. Even with a uniform thermal load provision, the server racks that are positioned near the two ends of the perforated tiles rows tend to be hotter compared to the ones in the middle of the tile rows. This is referred to end effect which is well-known in the data center industry. For resolving these issues, the tile airflow needs to be adjusted to overcome these drawbacks. An intentional tile airflow distribution in a specific arrangement is, therefore, the subject in this inverse design optimization.

Table IV.9. Targeted tile mass flow rate distribution at different shapes

\begin{tabular}{|l|c|c|c|c|c|c|c|c|c|}
\hline & \multicolumn{8}{|c|}{ Tile Mass Flow Distribution (kg/s) } & \multirow{2}{*}{$\begin{array}{c}\text { Total Mass } \\
\text { Flow (kg/s) }\end{array}$} \\
\hline \multicolumn{1}{|c|}{ Tile ID } & $\mathbf{1}$ & $\mathbf{2}$ & $\mathbf{3}$ & $\mathbf{4}$ & $\mathbf{5}$ & $\mathbf{6}$ & $\mathbf{7}$ & $\mathbf{8}$ & 12.0 \\
\hline Scenario 1 (Linear) & 0.66 & 0.90 & 1.14 & 1.38 & 1.62 & 1.86 & 2.10 & 2.34 & 12.0 \\
\hline Scenario 2 (Parabolic) & 2.70 & 1.67 & 0.99 & 0.64 & 0.64 & 0.99 & 1.67 & 2.70 & 12.0 \\
\hline Scenario 3 (Sinusoidal) & 0.50 & 2.50 & 0.50 & 2.50 & 0.50 & 2.50 & 0.50 & 2.50 & \\
\hline
\end{tabular}

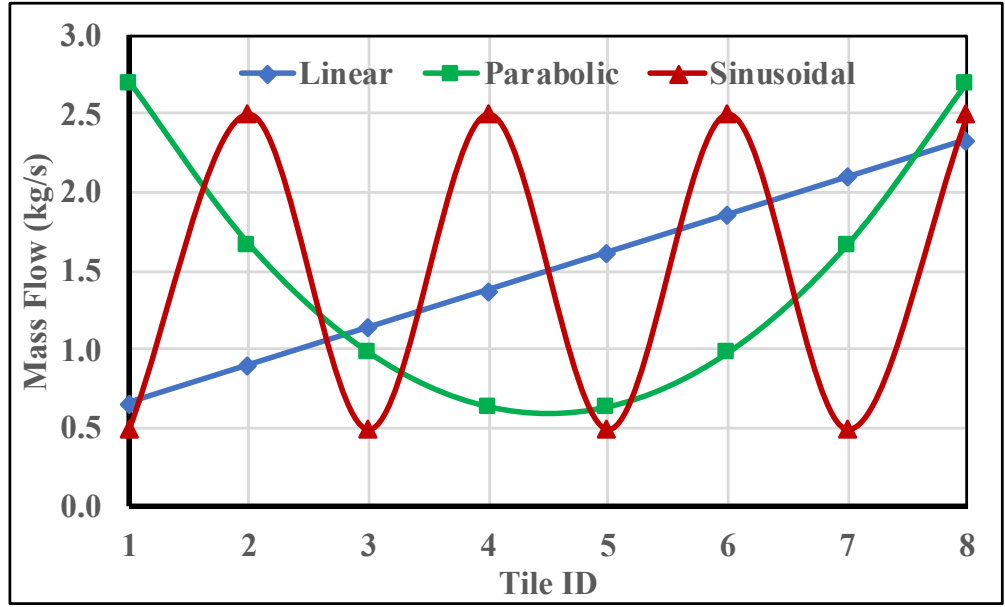

Fig. IV.22. Targeted shape for different tile mass flow rate distribution 
The idea is to use optimization to pick out the best tile porosity arrangement that satisfies the proposed airflow distribution objective. For demonstrating this concept, three airflow distribution scenarios in tiles are proposed, i.e., the linear, parabolic, and sinusoidal distributions as shown in Table IV.9. For all scenarios, the total mass flow rate through all 8 tiles are maintained at $12 \mathrm{~kg} / \mathrm{s}$ or 20,756 CFM. The respective chart for Table IV. 9 is

plotted in Fig. IV.22. The next three sections discuss the optimization processes similar to the uniformity design optimization with the objective function is the root mean square error (RMSE) defined in eq. (IV.13). An optimal design in each scenario is reached when the RMSE is less than 5\% compared to the proposed airflow distribution curve.

\section{a. Linear tile airflow distribution}

The first scenario of tile airflow distribution is the linear shape with a gradual increase in tile airflow from tile 1 through 8 . The optimization process to minimize the RMSE is similar to the uniformity design optimization where iterative stages are deployed to reach the final objective. A response surface was trained in each stage and assisted in the prediction of the designs. Performance measurement was also carried out to ensure the best suitable RBF model used. 


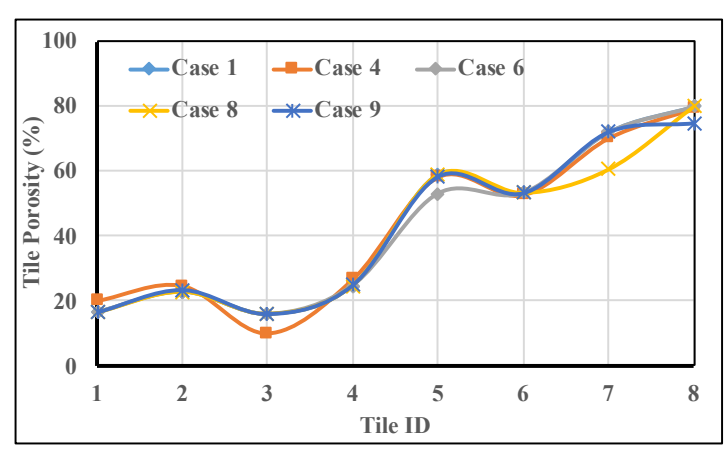

a) Stage 1

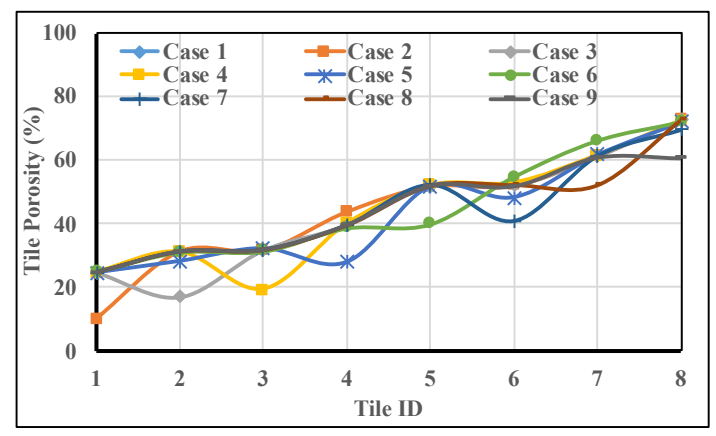

c) Stage 3

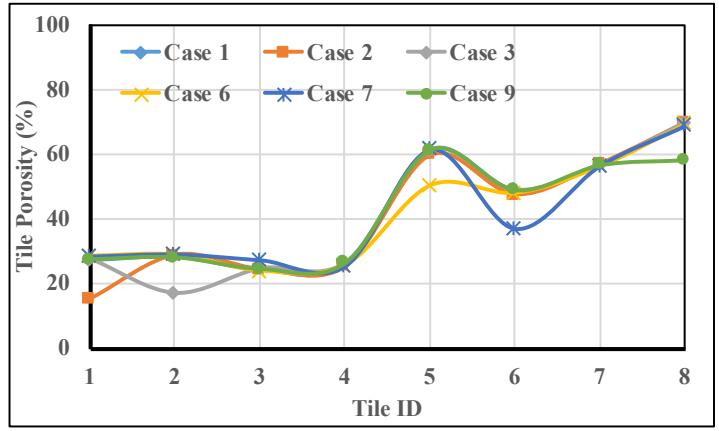

b) Stage 2

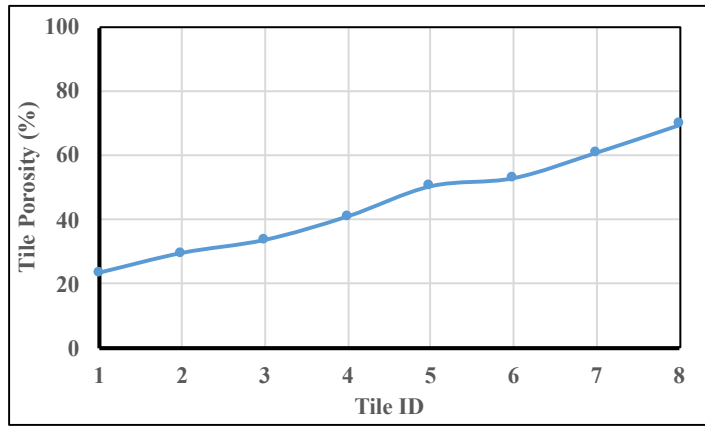

d) Stage 4

Fig. IV.23. Tile porosity for best-validated designs at each stage

Due to the space limit in the section, all of the charts describing the validation process at each stage can be retrieved from the appendix. Here, only the final designs after validation are presented. Fig. IV.23 shows the tile porosity for best validated designs at each stage. The validation process is very successful in overall, especially in stage 3 where all of the best designs suggested from the RSM-based optimization. The evolving progress of tile porosity throughout each stage is gradually shaped into a linear arrangement as seen Fig. IV.23. The final best design is presented in stage 4. 
Fig. IV.24 shows the tile mass flow rate for all best designs at each stage compared to the original and proposed linear airflow distribution designs. Clearly, it is shown that the design presented in stage 4 meets the expectation proposed with only $6 \%$ in RMSE compared to the proposed linear distribution. The contour plot in Fig. IV.24b also confirms the agreement with a relative improvement in RMSE of $90 \%$ of the optimized over the original designs when both are compared to the targeted linear airflow distribution line.
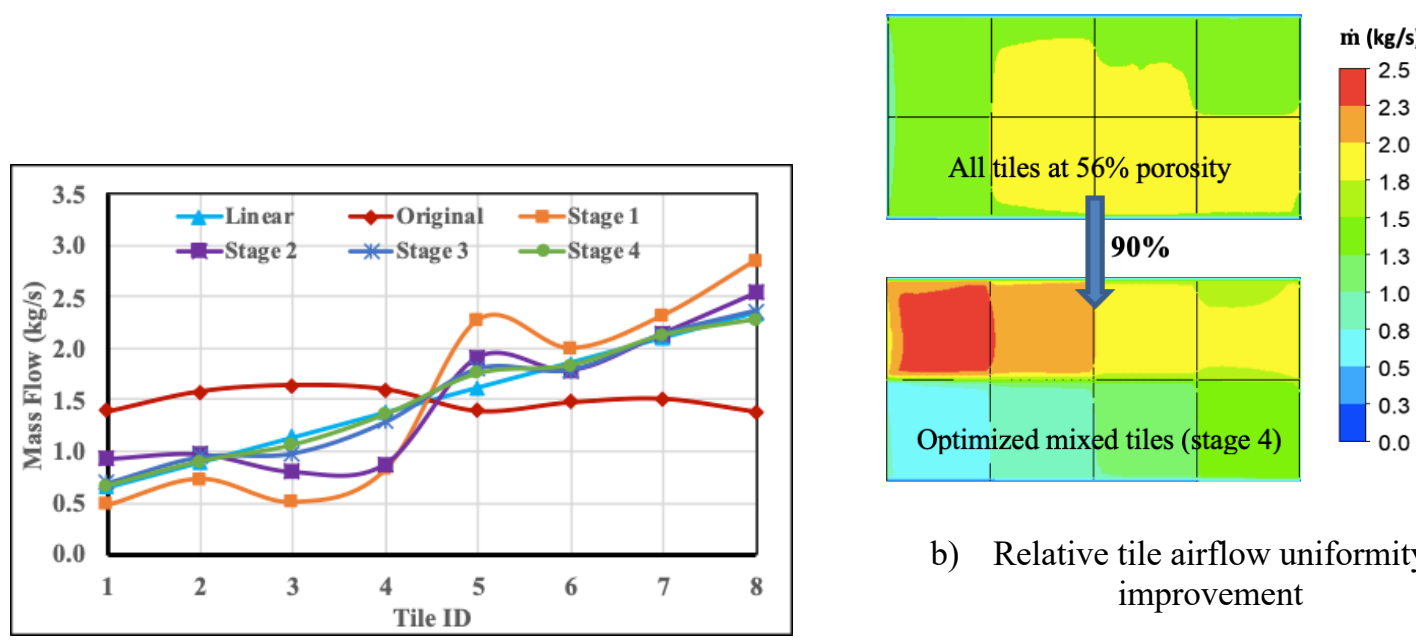

b) Relative tile airflow uniformity improvement

a) Tile mass flow rate for all best designs at each stage compared to the original and proposed linear airflow distribution designs

Fig. IV.24. Tile mass flow rate comparison

\section{b. Parabolic tile airflow distribution}

The whole optimization process for parabolic airflow distribution scenario is also identical to previous scenarios. The final results are shown in Fig. IV.25, while the validation demonstration can be referred to the appendix. Overall, the progress to the final best design 
presented in the final stage is very successful. Unlike the uniformity design optimization, the validation is much smoother in the inverse design optimization where many suggested best designs are well-validated and exceed the expectation. The reason is that the original design with uniform tile porosity of $56 \%$ has almost uniform airflow distribution in the tiles due to the symmetrical position of the CRAH unit placed in the data room. Therefore, there are little rooms for improvement in that case. For the inverse design optimization, a distinct airflow distribution shape is proposed which requires many more developments, as a result, the progress in each iterative stage shows a distinct improvement.

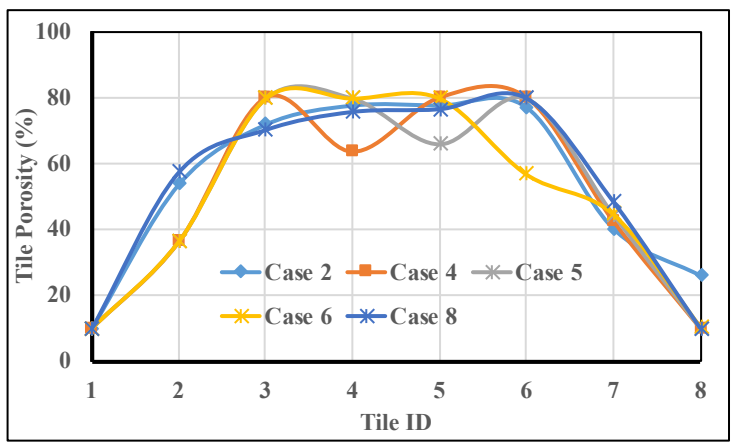

a) Stage 1

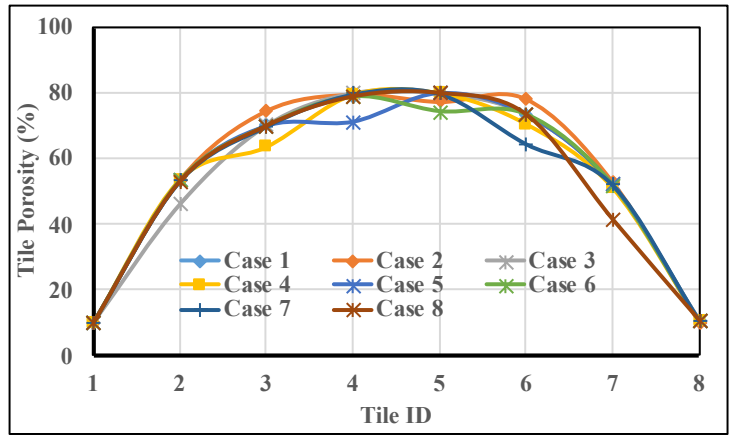

c) Stage 3

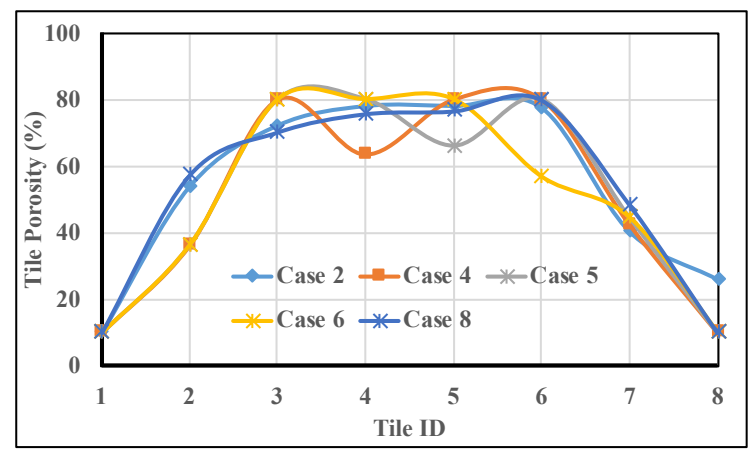

b) Stage 2

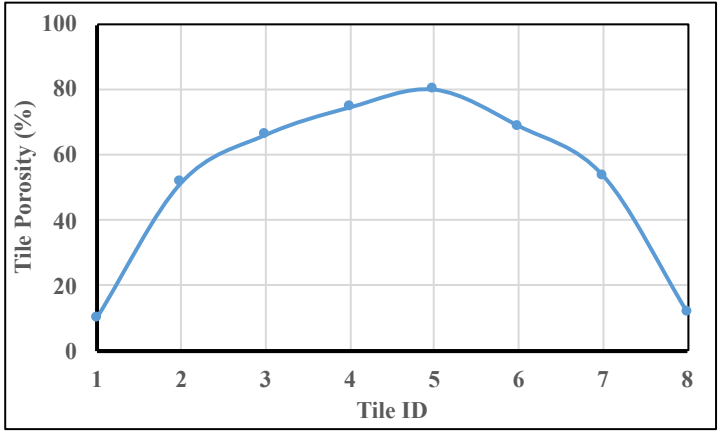

d) Stage 4

Fig. IV.25. Tile porosity for best-validated designs at each stage 
The progress to reach the final design that matches the proposed shape is demonstrated in Fig. IV.26. As expected, the final design in the last stage shows particularly good agreement with the proposed design ( $\mathrm{RMSE}=6 \%$ ). The relative improvement of $91 \%$ in RMSE of the optimized over the original designs is reflected in Fig. IV.26b, when both are compared to the proposed parabolic distribution line. Tiles $1 \& 8$ show the smallest mass flow rate, while tiles $5 \& 6$ receive the peak airflow rate.

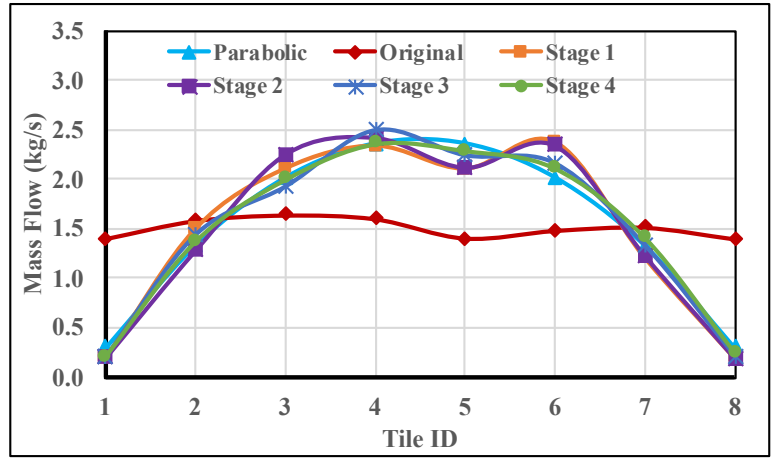

a) Tile mass flow rate for all best designs at each stage compared to the original and uniform cases

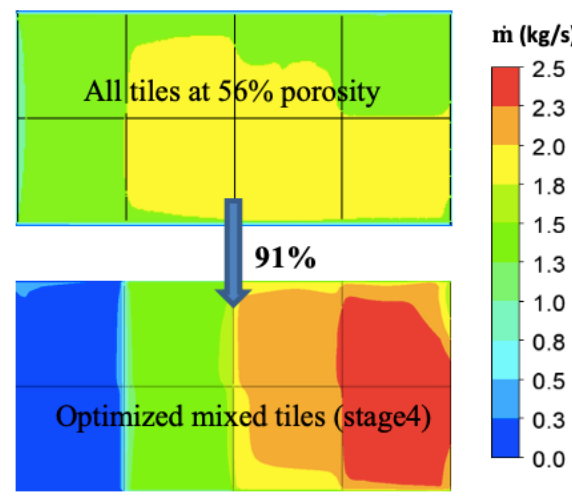

b) Relative tile airflow uniformity improvement

Fig. IV.26. Tile mass flow rate comparison

\section{c. Sinusoidal airflow distribution}

Lastly, the sinusoidal airflow distribution shape explored in this section is an extreme condition which the tiles are undergoing an oscillating form of airflow rate. The validation process can be referred to the appendix. Here, the final optimal designs in each iterative stage are observed. Fig. IV.27 shows best designs selected at each iterative stage. 
Fig. IV.28 shows the progress toward the proposed objective. The best design in stage 4 matches well with the proposed shape with the RMSE only $5.9 \%$. It is also shown that the original design with uniform porosity of $56 \%$ cannot create an alternating trend in tile airflow as compared to the optimized design in this work, and Fig. IV.28b clearly shows this comparison with a relative improvement in RMSE of $94 \%$ of the optimized over the original designs, when both are compared to the proposed sinusoidal distribution line.

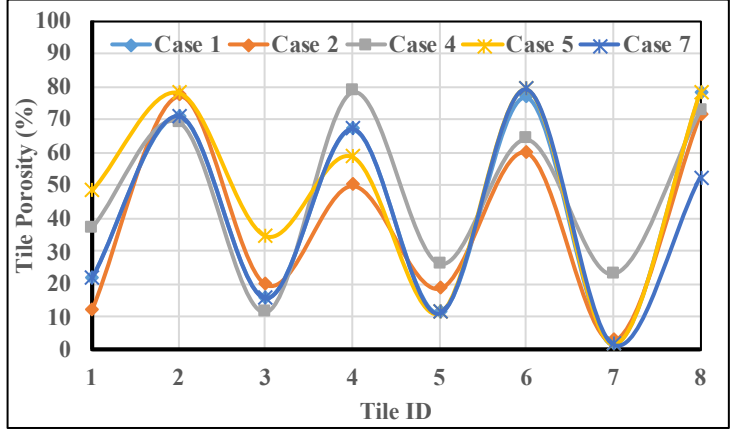

a) Stage 1

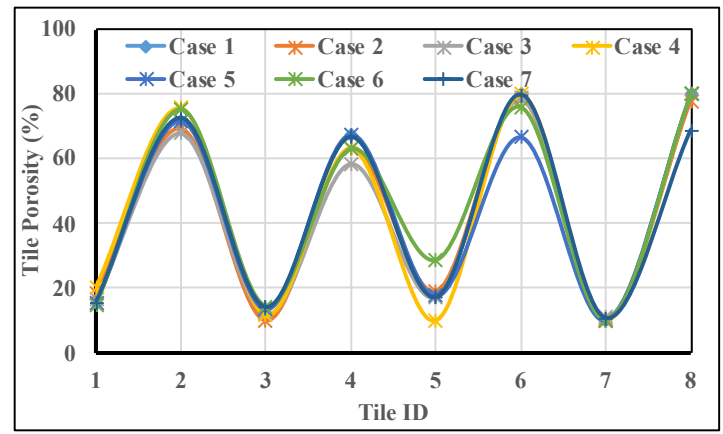

c) Stage 3

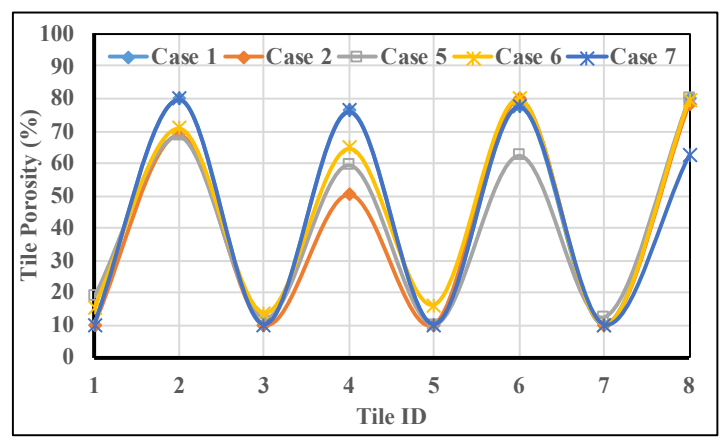

b) Stage 2

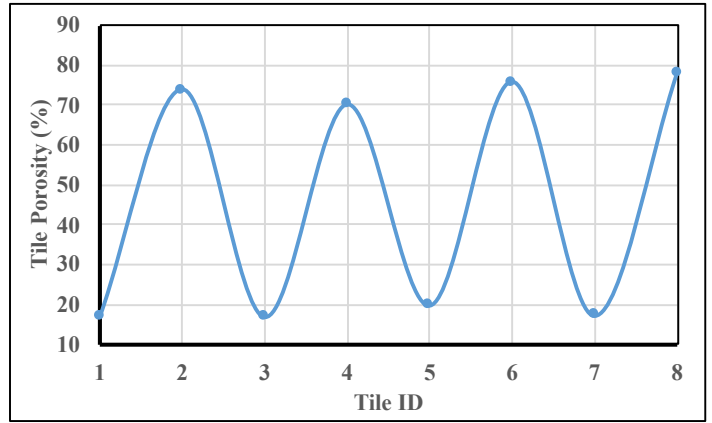

d) Stage 4

Fig. IV.27. Tile porosity for best-validated designs at each stage

In addition to reaching all design objectives for both the uniformity design and the three inverse designs, the time required for the optimization processes is also advantageous 
aspect when using RSM. In fact, if CFD method was to be used instead of relying on the response surfaces, the total time spent for generating all predicted designs during an optimization process takes 9000 hours or 375 days since each simulation costs approximately 2 hours of running time and there are 4500 predicted designs generated with GA. This is based on a 7-core personal Dell computer with Intel ${ }^{\circledR}$ Core $^{\mathrm{TM}}$ i7-4770 CPU at $3.4 \mathrm{GHz}$ with $32 \mathrm{~GB}$ of memory. For a response surface trained at each iterative stage, the total time for obtaining the predicted designs is only a few seconds. Overall, the total time required for the entire design process from preparing the original data set to the complete final design is outlined in Table IV.10. This proves a substantial time reduction when using RSM for optimization processes. Moreover, the RSM can be used in conjunction with experimental testing at each iterative design stage to increase the level of accuracy and fidelity of the entire design method.

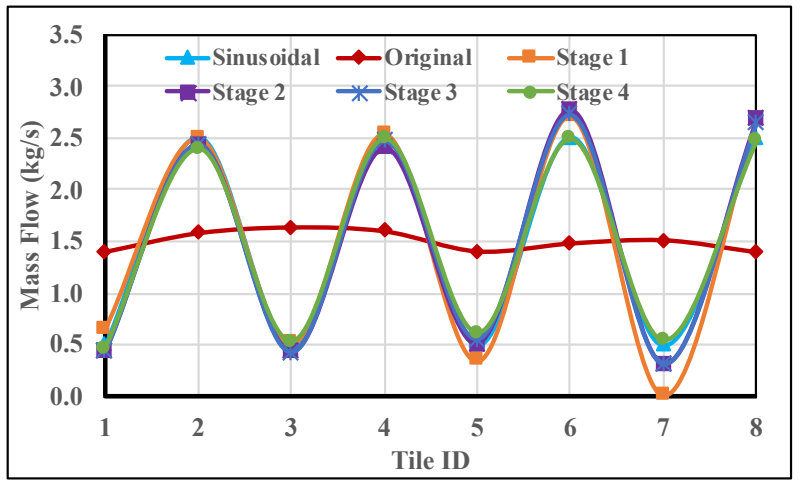

a) Tile mass flow rate for all best designs at each stage compared to the original and uniform cases

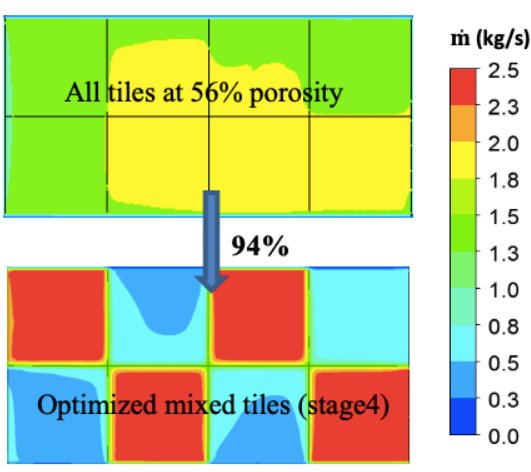

b) Relative tile airflow uniformity improvement

Fig. IV.28. Tile mass flow rate comparison 
Table IV.10. Total time comparison between CFD and RSM methods.

\begin{tabular}{|c|c|c|}
\hline Tasks & Design optimization without RSM & Design optimization with RSM \\
\hline Original data set & 45 cases x 2 hrs./case $=90$ hours & 45 cases x 2 hours/case $=90$ hrs. \\
\hline Optimization & 4500 designs x $2 \mathrm{hrs} /$ design $=9000$ & RSM training: a few seconds \\
process & hrs. & 40 hrs. \\
\hline Total & $\sim 9090 \mathrm{hrs}$. or $~ 379$ days & $\sim 170$ hrs. or $\sim 7$ days \\
\hline
\end{tabular}

\subsection{Remarks}

In this chapter, response surface methodology based on radial basis function is studied for the application of thermal management in data centers. RSM shows a significant reduction in running time compared to a CFD simulation, and therefore is suitable during an optimization process. For demonstration, RSM is used to aid in an optimization effort to seek an optimal arrangement of mixed-porosity tiles for either a uniform (uniformity design optimization) or a specific (inverse design optimization) tile airflow distribution. Details of the RSM-based optimization through iterative stages are demonstrated including the response surface training, performance assessment, as well as validation with CFD simulated results. Other important advantage is the capability to rapidly reconstruct the temperature profile, which makes it possible for near real-time thermal analysis of data centers.

In the first application, RSM was utilized to reconstruct the temperature profile inside a data center model. Three different scenarios are presented, i.e., $5 \%, 10 \%$, and $20 \%$ of the original data points reserved as training points for response surface construction. The 
advantage of using response surface is clearly excellent. By using a small set of training points, we can construct a response surface (hypersurface) to interpolate other missing points with accepted accuracy. It is extremely useful for real life situation since limited probes are used in experiment. With a certain experimental data points, one can construct the response surface and interpolate other missing data point within the working domain. Among the three predicted cases, case $20 \%$ shows higher accuracy as compared to others. With only $20 \%$ of the original data used for training the response surface, the case can capture most of the aspects of the original CFD model.

In the second application, RSM is utilized in a multi-objective optimization process that uses Genetic Algorithm as its engine. Both the temperature difference and the maximum temperature inside the data center are minimized for a reduction of the energy use and a provision of a healthy environment for the servers. A range of design parameters including the mass flow inlet, the inlet temperature, and the server heat load are optimized to search for the best combinations that satisfy both objectives. A detailed procedure for RSM surface training is shown, and a final set of Pareto designs are obtained. These designs are equally good and non-dominant of each other in terms of the studied two objectives. It is found that for an inlet temperature range and an inlet mass flow rate range of $11.8-$ $12.62^{\circ} \mathrm{C}, 7.36-7.67 \mathrm{~kg} / \mathrm{s}$, respectively, the two objectives are equally achieved. The Pareto designs are validated against high fidelity $\mathrm{CFD} / \mathrm{HT}$ results and show very good agreement.

In both the uniformity design and the inverse design optimization processes, the method successfully suggests better arrangements of mixed-porosity tiles over the original design 
of deploying all uniform tiles at $56 \%$ porosity in data centers. In fact, the relative improvement over the original design is $55 \%$ in terms of standard deviation in the uniformity design optimization, whereas it is 90\%, 91\%, and 94\% in RMSE for the linear, parabolic, and sinusoidal tile airflow distributions, respectively, in the inverse design optimization. Without relying on CFD method for generating virtual designs, the RSM shows a substantial time reduction at each iterative stage during an optimization process. With the available tool for tile porosity adjustment, the method presents an innovative technique that allows better arrangements of mixed-porosity tiles over traditional uniform tiles practice to provide maximum cold-air to server racks with or without uniform thermal loadings. As a result, much cooling energy can be saved toward a more energy-efficient data center. 


\section{CHAPTER V ENERGY MODELING}




\subsection{Data Center Modeling}

In this chapter, a new multi-zone modeling strategy was proposed for a popular data center model that has both hot and cold aisles. A parametric study was conducted to study the effects of five different surface boundary conditions (surface exposures) on the cooling electricity consumption and the zone mean air temperature of the data center. The studies were carried out for two typical U.S. climates, very hot and humid (Miami, FL), cool and humid (Chicago, IL). In addition, both supply temperature and volumetric flow rate of the supply side were also investigated to seek the optimal operating conditions in a representative city, Miami, Florida.

\subsubsection{Data Center Description}

The data center model (Fig. II.1) continues to be investigated in this study. The CRAC units supply cooling air at $55 \mathrm{~F}\left(12.8^{\circ} \mathrm{C}\right)$ from the pressurized 2 -foot raised floor plenum. The data center was configured using the current best practice of hot and cold aisles. The server racks are placed in such a way that their back sides are facing each other to form hot aisles, while the front sides, which receive cooling air from the perforated floor tiles, form cold aisles. The load density of the data center model consisting of IT equipment and lighting load was specified to be $100 \mathrm{~W} / \mathrm{ft}^{2}$ at all time (totally 8760 hours a year) based on a previous study by Hong et al. [33]. The HVAC system used was a packaged single zone (PSZ) direct expansion (DX) system with air-cooled condenser, constant supply fan, and without humidity control as well as air-side economizer. Similar to the load schedule, the fan schedule was also assumed to be on at all time. The current geometrical model was created 
using Google Sketchup tool [28], then transferred to EnergyPlus [23] to perform building energy simulation.

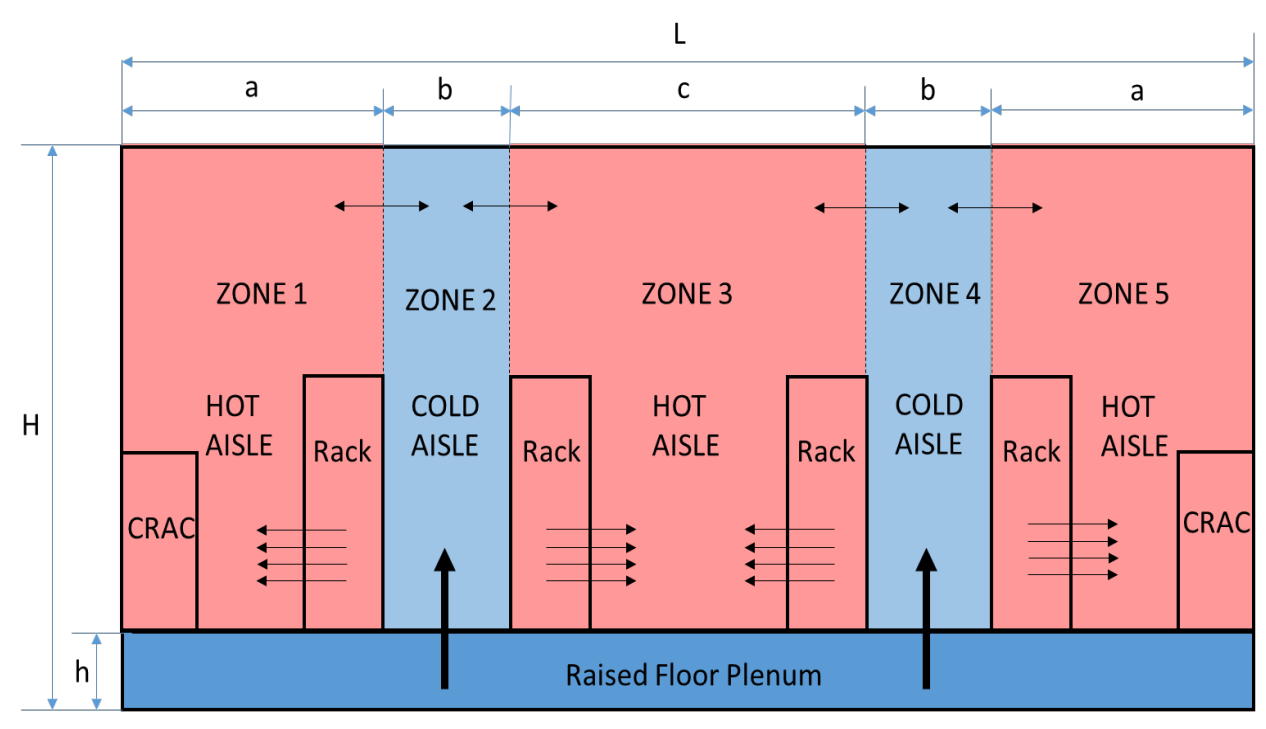

Fig. V.1 Multi-Zone Data Center Model

The main focus of this chapter is to use a proposed multi-zone model which will be described in the next section to separate the computer room into subzones, i.e., hot and cold aisles. We have proposed to use five zones ( 3 hot and 2 cold zones) for the data center model, as seen in Fig. V.1. Zone 2 and 4 were specified as cool zones with no loads. Zone 1 and 5 were assigned a thermal load equals to $29,400 \mathrm{~W}$. Finally, zone 3 (middle zone) was assigned a thermal load of 58,800 Watts since this zone holds up to two rows of rack unit. These figures are equivalent to a load density of $100 \mathrm{~W} / \mathrm{ft}^{2}\left(1076.4 \mathrm{~W} / \mathrm{m}^{2}\right)$. Volume subtraction was used to account for the occupied space of the servers, as well as the CRAC units. Therefore, the computational volume is less than the total volume of the entire data center. Interzone air mixing was properly handled by applying ZRDM object to ensure that 
air is interchangeable among the subzones. Weather information of Miami and Chicago was obtained through the database supported by EnergyPlus website.

Five building setups were investigated to study the effect of boundary conditions. Detailed boundary condition information is listed in Table V.1. In order to emphasize on the wall boundary condition and location effects rather than construction materials, all the building material used to construct these five cases for both locations were identical based on the generic material in EnergyPlus database as shown in Table V.2, although the construction materials might be different for different states.

Table V.1 Various surface exposure conditions of the data center model

\begin{tabular}{|c|l|l|l|l|l|}
\hline $\begin{array}{c}\text { Boundary } \\
\text { Condition }\end{array}$ & \multicolumn{1}{|c|}{ All } & \multicolumn{1}{c|}{ Roof } & Roof and Ground & \multicolumn{1}{c|}{ Side Walls } & \multicolumn{1}{c|}{ Floor } \\
\hline Description & $\begin{array}{l}\text { All surfaces are } \\
\text { exposing to } \\
\text { outside weather }\end{array}$ & $\begin{array}{l}\text { Only roof } \\
\text { surface is } \\
\text { exposing to the } \\
\text { outside weather }\end{array}$ & $\begin{array}{l}\text { Only roof and } \\
\text { ground surfaces } \\
\text { are exposing to } \\
\text { the outside } \\
\text { weather }\end{array}$ & $\begin{array}{l}\text { Only side walls } \\
\text { are exposing to } \\
\text { the outside } \\
\text { weather }\end{array}$ & $\begin{array}{l}\text { Only the floor } \\
\text { surface is } \\
\text { exposing to the } \\
\text { ground }\end{array}$ \\
\hline
\end{tabular}

Table V.2 Prescribed construction set for the current data center model

\begin{tabular}{|c|c|c|c|c|c|c|c|}
\hline & $\begin{array}{c}\text { Interior } \\
\text { Ceiling }\end{array}$ & IRT Surface & Interior Floor & Exterior Slab & Interior Wall & $\begin{array}{c}\text { Exterior } \\
\text { Wall }\end{array}$ & $\begin{array}{c}\text { Exterior } \\
\text { Roof }\end{array}$ \\
\hline $\begin{array}{c}\text { Layer } \\
1\end{array}$ & $\begin{array}{l}\text { Lightweight } \\
\text { concrete } \\
(100 \mathrm{~mm})\end{array}$ & $\begin{array}{l}\text { IRT material } \\
\quad(0 \mathrm{~mm})\end{array}$ & $\begin{array}{l}\text { Acoustic tile } \\
(19.1 \mathrm{~mm})\end{array}$ & $\begin{array}{l}\text { Concrete } \\
(100 \mathrm{~mm})\end{array}$ & $\begin{array}{l}\text { Gypsum } \\
\text { board } \\
(19 \mathrm{~mm})\end{array}$ & $\begin{array}{c}\text { Brick } \\
(100 \mathrm{~mm})\end{array}$ & $\begin{array}{c}\text { Exterior } \\
\text { roof } \\
\text { material } \\
(100 \\
\mathrm{mm})\end{array}$ \\
\hline $\begin{array}{c}\text { Layer } \\
2\end{array}$ & $\begin{array}{l}\text { Ceiling air } \\
(30 \mathrm{~mm})\end{array}$ & & $\begin{array}{l}\text { Ceiling air } \\
(30 \mathrm{~mm})\end{array}$ & $\begin{array}{c}\text { Carpet Pad } \\
(10 \mathrm{~mm})\end{array}$ & $\begin{array}{l}\text { Wall air } \\
\text { space } \\
\text { resistance } \\
(15 \mathrm{~mm})\end{array}$ & $\begin{array}{l}\text { Heavyweight } \\
\text { concrete } \\
(200 \mathrm{~mm})\end{array}$ & $\begin{array}{c}\text { Ceiling } \\
\text { air } \\
(30 \mathrm{~mm})\end{array}$ \\
\hline $\begin{array}{c}\text { Layer } \\
3\end{array}$ & $\begin{array}{c}\text { Acoustic } \\
\text { tile } \\
(19.1 \mathrm{~mm})\end{array}$ & & $\begin{array}{l}\text { Lightweight } \\
\text { concrete } \\
(100 \mathrm{~mm})\end{array}$ & & $\begin{array}{l}\text { Gypsum } \\
\text { board } \\
(19 \mathrm{~mm})\end{array}$ & $\begin{array}{l}\text { Insulation } \\
\text { board } \\
(50 \mathrm{~mm})\end{array}$ & $\begin{array}{c}\text { Acoustic } \\
\text { tile } \\
(19.1 \\
\mathrm{mm}) \\
\end{array}$ \\
\hline $\begin{array}{c}\text { Layer } \\
4\end{array}$ & & & & & & $\begin{array}{l}\text { Wall air } \\
\text { space } \\
\text { resistance } \\
(15 \mathrm{~mm})\end{array}$ & \\
\hline $\begin{array}{c}\text { Layer } \\
5\end{array}$ & & & & & & $\begin{array}{l}\text { Gypsum } \\
\text { board } \\
(19 \mathrm{~mm})\end{array}$ & \\
\hline
\end{tabular}




\subsubsection{Single Zone Model}

For comparison purpose, the conventional single zone model was used in the data center modeling. The single zone model is usually modeled with the assumption that air within a zone is well-mixed. Therefore, zone air temperature is basically uniform throughout the entire room. Normally, this corresponds to the desired physical state of the air within a zone. Practically, this is accomplished with the mixing caused by the air distribution system or even with the addition of ceiling fans. However, there are situations where the wellstirred model condition does not fit the physical conditions within a space (e.g., large atria spaces, displacement ventilation systems, and under floor air distribution systems). In these cases, the air temperature varies spatially within the zone, but the surfaces in the regions that have different air temperatures still exchange IR radiation with the other surfaces in the zone, even those in regions having a different air temperature.

\subsubsection{Multi-zone Model}

The proposed multi-zone model used in this study was constructed for the data center using the infrared transparent (IRT) model. That is, the space is divided into subzones, each having the basic well-stirred air model, but surfaces in these sub-zones are also exchanging infrared (IR) radiation with other surfaces throughout the original space. In other words, the sub-zones are standard EnergyPlus zones but they have been given the capability of allowing IR radiation to be exchanged with surfaces in adjacent zones. 
In the present study, the IRT surface was used in the multi-zone models to divide the computer room in several sub-zones representing the hot and cold aisles. Since the IRT surface is acting as a virtual wall (air wall) to divide the zone into subzones, its surface should not participate in a convective/conductive exchange between the zones it separates. In order to minimize this effect, the outside and inside values for the surface's convection coefficients should be on the order of $0.1 \mathrm{~W} / \mathrm{m}^{2} \mathrm{~K}$. However, it allows the IR radiation exchange among the surfaces within the computer room zone. Although the air temperature varies spatially within the zone, the surfaces in the regions that have hotter air temperatures, i.e., hot aisles, will exchange IR radiation with the colder surfaces, i.e., cold aisles.

\subsubsection{Description of CFD Flow Modeling}

For validation purpose, CFD simulation is also carried out in this study. In the CFD modeling, the standard $k$ - $\varepsilon$ turbulence model was used. The following turbulence model constants were used $C_{\mu}=0.09, C_{1 \varepsilon}=0.09, C_{2 \varepsilon}=1.92$, and turbulence kinetic energy Prandtl number $=1.0$. Standard wall function was used in this turbulence model. The grid was generated in such a way that the $y+$ values meet the recommended range for the standard turbulence model. For the velocity-pressure coupling, the phase coupled SIMPLE algorithm was selected, while least squares cell based was used for gradient in spatial discretization. Upwind schemes were applied for momentum, volume fraction, turbulent kinetic energy, and turbulent dissipation rate equations. Numerical computations were carried out using the commercial CFD package [6]. The convergence criteria are set as less than $10^{-5}$ in residuals for all computed variables. 


\subsection{Fundamental of Energy Modeling}

Building energy simulation programs are essentially developed based on energy balance equations for zone air and surface heat transfer inside a control volume or a room air model. In EnergyPlus, the following ordinary differential equations are solved

$$
C_{z} \frac{d T_{z}}{d t}=\sum_{i=1}^{N_{s l}} \dot{Q}_{\imath}+\sum_{i=1}^{N_{\text {surfaces }}} h_{i} A_{i}\left(T_{s i}-T_{z}\right)+\sum_{i=1}^{N_{z o n e s}} \dot{m}_{\iota} C_{p}\left(T_{z i}-T_{z}\right)+\dot{m}_{\text {inf }} C_{p}\left(T_{\infty}-T_{z}\right)+\dot{Q}_{s y s}
$$

where, $\sum_{i=1}^{N_{s l}} \dot{Q}_{l}=$ sum of the convective internal loads, $\sum_{i=1}^{N_{\text {surfaces }}} h_{i} A_{i}\left(T_{s i}-T_{z}\right)=$ convective heat transfer from the zone surfaces, $\dot{m}_{\text {inf }} C_{p}\left(T_{\infty}-T_{z}\right)=$ heat transfer due to infiltration of outside air, $\sum_{i=1}^{N_{\text {zones }}} \dot{m}_{l} C_{p}\left(T_{z i}-T_{z}\right)=$ heat transfer due to interzone air mixing, $\dot{Q}_{s y s}=$ air systems output, $C_{z} \frac{d T_{z}}{d t}=$ energy stored in the zone air, $C_{z}=\rho_{a i r} C_{p} C_{T}$.

Typically, the capacitance $C_{z}$ would be that of the zone air only. However, thermal masses assumed to be in equilibrium with the zone air could be included in this term. In Eq. (V.1), the convective heat transfer coefficient, $h_{i}$, is unknown. Most energy programs estimate $h_{i}$ by empirical equations or as a constant. In EnergyPlus, it is modeled from a choice of correlations. Air system control in EnergyPlus is based on the calculation of how much energy enters or leaves the zones as a function of zone air temperature, i.e., the zone load. The net zone load is given by

$$
\dot{Q}_{\text {load }}=\sum_{i=1}^{N_{s l}} \dot{Q}_{i}+\sum_{i=1}^{N_{\text {surfaces }}} h_{i} A_{i}\left(T_{s i}-T_{z}\right)+\sum_{i=1}^{N_{\text {zones }}} \dot{m}_{i} C_{p}\left(T_{z i}-T_{z}\right)+\dot{m}_{\text {inf }} C_{p}\left(T_{\infty}-T_{z}\right)
$$


In addition, $T_{z}$ is now the desired zone temperature as defined by the control system setpoints that must be specified for each zone. An assumption was made that if the air system has sufficient capacity (based on the desired zone air temperature) to meet the zone

conditioning requirements (i.e. $\dot{Q}_{s y s}=\dot{Q}_{l o a d}$ ) at the desired zone air temperature then those requirements will be met.

Typically, a single zone draw through air system has a cooling coil and heating coil in series, and constant air volume flow rate. Single zone draw-through systems run at maximum capacity when turned on; so the only way to regulate net air system output and keep the zone air temperature within the desired range is to turn the air system on and off. The amount of heating or cooling provided by the air system in relation to the desired zone air temperature is given by

$$
\dot{Q}_{s y s}=\dot{m}_{s y s} C_{p} \eta\left(T_{\text {sup }}-T_{z, \text { desired }}\right)
$$

where $\eta$ is the fraction of the time step that the air system is turned on and varies between 0 and 1 . The supply air temperature is also implicitly limited by the effectiveness of the coils and the operating parameters of the central plant component.

\subsection{Results and Discussion}

\subsubsection{Verification Study}

In Hong's paper [33], a simple single zone model was used to isolate the complexity of building loads and other end uses so that the focus is on the HVAC system performance. First, the present study tried to replicate the simulation process as done by Hong et al. to 
make sure the result was consistent. To this end, a rectangular box with dimensions reported by Hong et al., as shown in Fig. V.2, was simulated. Fig. V.3 shows the calculated monthly cooling load and different types. As shown from the figure, the current result of the replicated simulation perfectly matched Hong's results. This granted confidence of utilizing EnergyPlus in the current study.

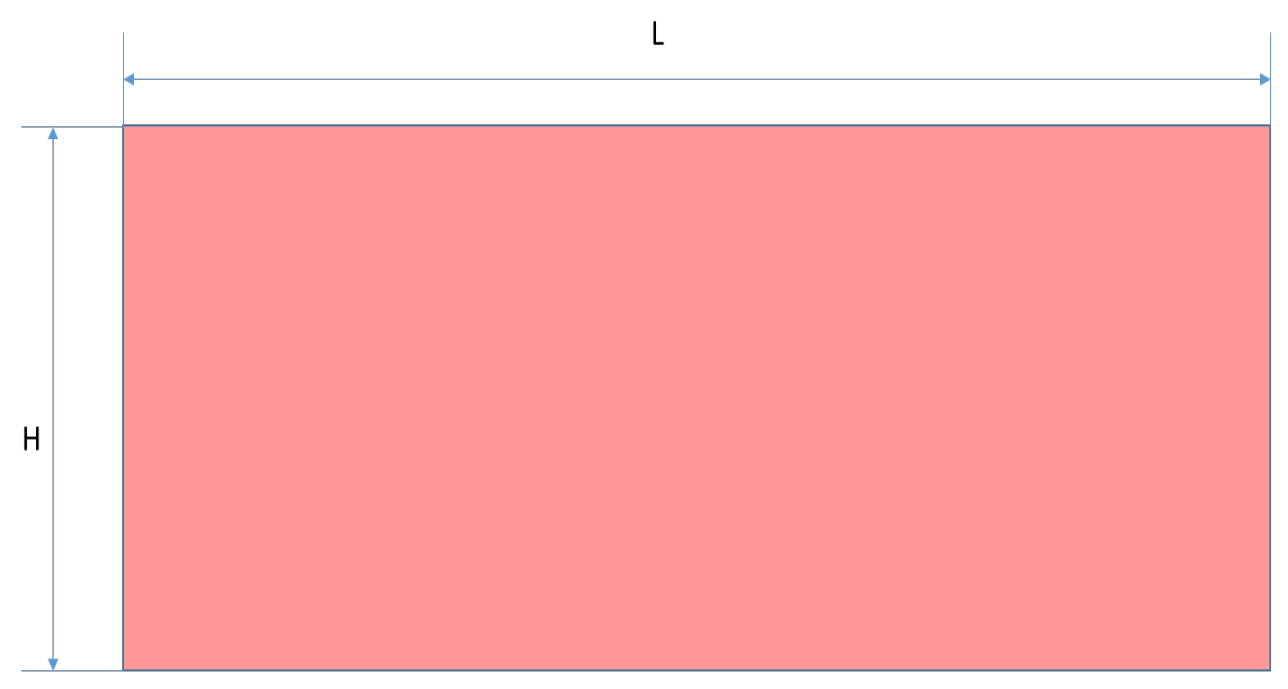

Fig. V.2 Single-Zone Data Center Model

Fig. V.4 shows the validation between the CFD and EnerguPlus simulation results for the data center with adiabatic walls. In Fig. V.4a, the result for multi-zone approach is very close to the CFD simulation, while the singe zone temperature result remains constant throughout computer room. Apparently, the multi-zone approach resolves the temperature profile better than the single zone approach when compared with CFD. Also, the average room temperatures for $\mathrm{CFD}$, multi-zone, and single zone are calculated to be $25.50^{\circ} \mathrm{C}$, $23.67^{\circ} \mathrm{C}$, and $20.60^{\circ} \mathrm{C}$, respectively. The multi-zone approach shows only $7 \%$ average zone temperature difference with CFD, while it is $19 \%$ for single zone approach. Fig. V.4b 
further shows the temperature profile of the computer room in the middle plane across the entire data center.

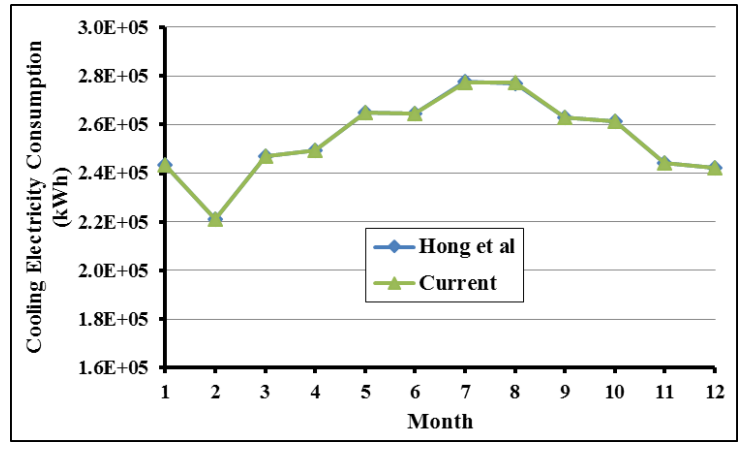

a) Monthly Cooling Load

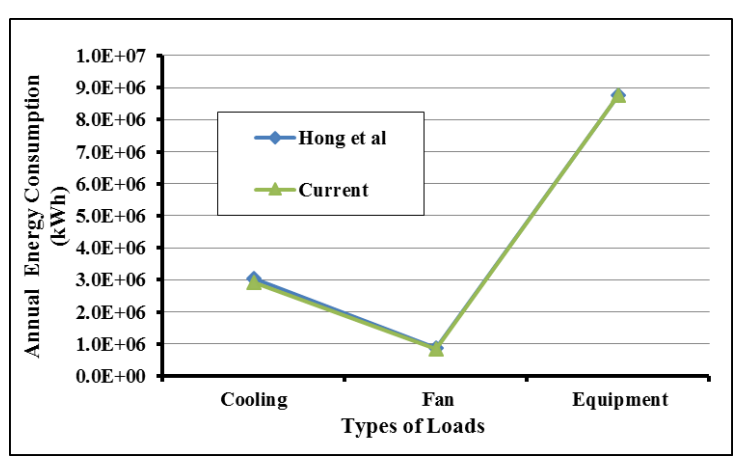

b) Types of Load

Fig. V.3 Hong's paper verification

Fig. V.5 shows the comparison between single zone (baseline) and multi zone models. Both Miami and Chicago cases are presented in this study. In Fig. V.5a, it is clear that there is a big difference between two approaches. The multi-zone approach suggests that the monthly cooling electricity consumption has a peak of $27,500 \mathrm{kWh}$, while it is $33,000 \mathrm{kWh}$ in the case of single zone model. Similarly, in Fig. V.5b, it is in a peak of $31,000 \mathrm{kWh}$ for single zone approach and 26,000 kWh for multi-zone approach. The average percent difference between two approaches for Miami is $16 \%$ and $14 \%$ for Chicago as seen in Table V.3. Although these figures were not validated with real experimental data, the CFD simulation results from Fig. V.4 suggest that multi-zone approach for data centers is much closer to resolve the real situation's energy consumption. In fact, the closer temperature agreement from multi-zone approach with CFD results suggests that energy consumption in the multizone model has a strong reliability compared to single zone model. Also, the 19\% 
temperature difference between CFD and single zone results (Table V.4) emphasizes the invalidity of the single model compared to the multi-zone model. However, further experimental work needs to be carried out to fully confirm the usefulness of the multi-zone approach. The percentage difference of temperature for CFD and multi-zone as well as multi-zone and single zone are $7 \%$ and $13 \%$, respectively.

Table V.3 Multi-zone and single zone comparison in Miami and Chicago

\begin{tabular}{|c|c|c|c|}
\hline \multicolumn{3}{|c|}{ Cooling Electricity Consumption (kWh) } \\
\hline Location & Multi-zone (E+) & Single Zone (E+) & Deviation (\%) \\
\hline Miami & 25,542 & 30,450 & $16 \%$ \\
\hline Chicago & 23,883 & 27,691 & $14 \%$ \\
\hline
\end{tabular}

Table V.4 Comparison between two methods with CFD for adiabatic condition

\begin{tabular}{|c|c|c|c|}
\hline \multicolumn{2}{|c|}{ Temperature $\left({ }^{\circ} \mathrm{C}\right)$} & \multicolumn{2}{c|}{ Deviation (\%) } \\
\hline Single zone & 20.60 & CFD vs. Single & $19 \%$ \\
\hline Multi-zone & 23.67 & CFD vs. Multi & $7 \%$ \\
\hline CFD & 25.50 & Multi vs. Single & $13 \%$ \\
\hline
\end{tabular}
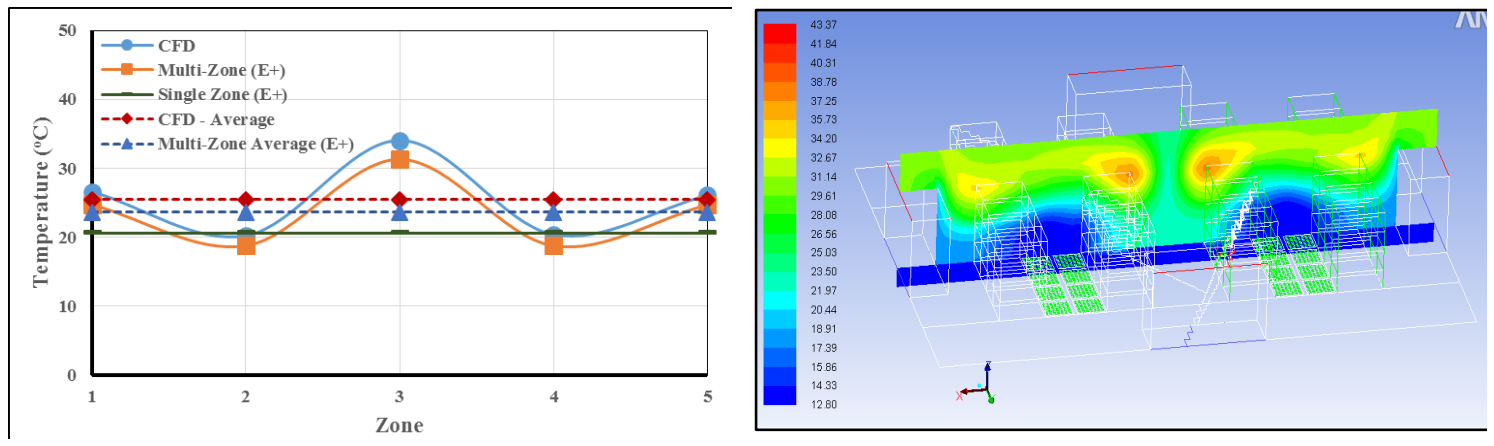

a) Zone mean air temperature comparison between CFD and energy plus (adiabatic condition)
b) Zone mean air temperature result of the multi-zone model approach using CFD

Fig. V.4 CFD and Energy Plus validation for the multi-zone model approach

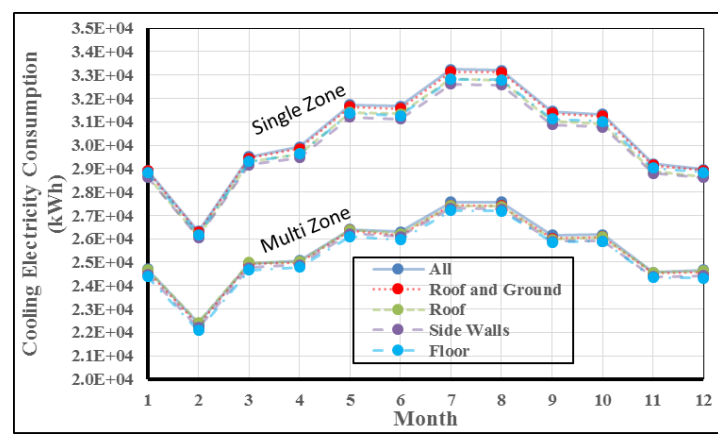

a) Miami

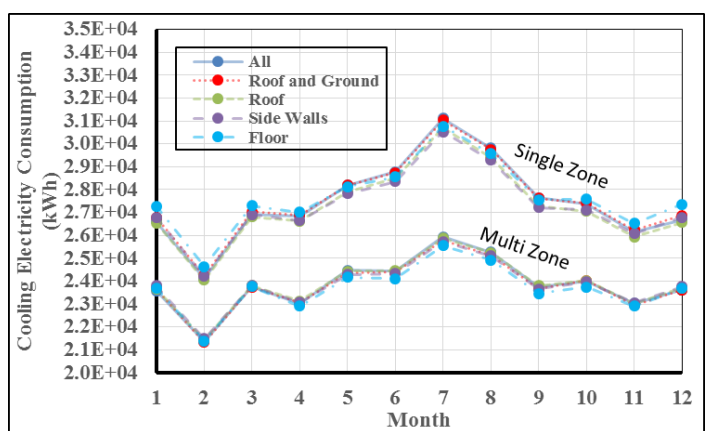

b) Chicago

Fig. V.5 Monthly cooling electricity consumption for single zone and multi zone

\subsubsection{Effect of Location}

Fig. V.6 shows monthly cooling electricity consumption between Miami and Chicago. Generally, it is higher in Miami than in Chicago due to the fact that Chicago is cooler compared to Miami. Therefore, the HVAC system needs less power to run in Chicago than Miami. In terms of boundary conditions, Roof and Ground exposure shows a greater impact than Side Walls exposure. Clearly, the cases where the outside surfaces of the data center are any combination of roof exposure tend to consume more electricity (these cases include: All, Roof, and Roof and Ground exposures). When only the floor surface is connected to the ground, the data center consumes least cooling power in both locations. The location factor plays an important role to decide whether the boundary condition is 
significant throughout the months. Separation among the boundary conditions in Chicago is typically smaller than Miami. It is higher in summer months for both locations. However, in the case of Chicago, the lines are almost overlapping in winter and the line separation is relatively smaller in summer compared to Miami. This indicates that boundary condition factor has a lesser effect on monthly cooling electricity consumption of the data center in chiller climate.

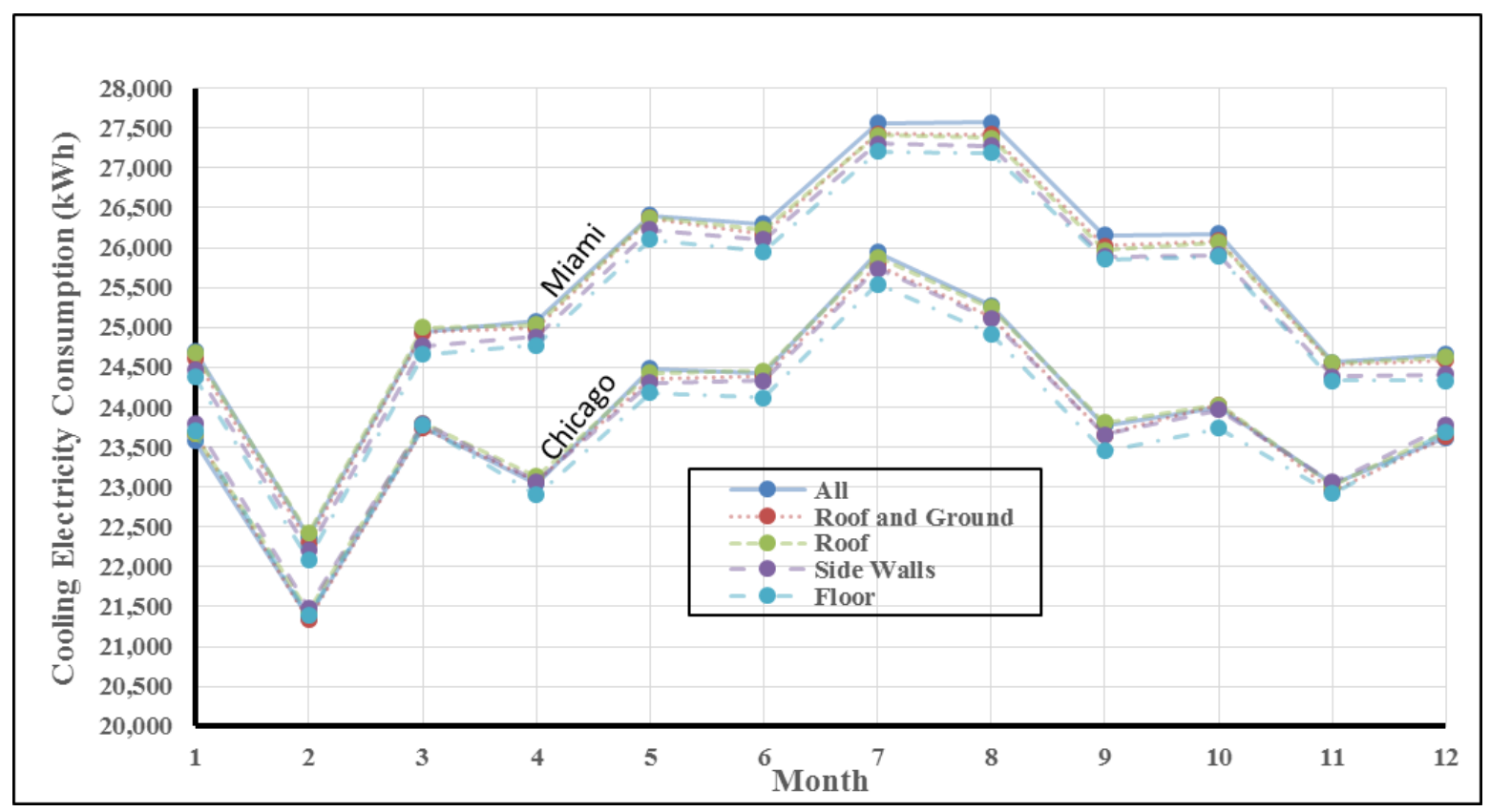

Fig. V.6 Monthly cooling electricity consumption between Miami and Chicago

Fig. V.7 shows the zone mean air temperature at different subzones inside the data center for Miami and Chicago, respectively. Zones $2 \& 4$ are representing the cold aisles; zones 1,3 , and 5 are the hot aisles zones. Zone 3 has the hottest temperature since it receives hot air from both sides of the data center racks aligned back to back. The heat loads are assigned to be constant for each server rack. Therefore, the zone mean air temperature profile is 
essentially symmetrical. In Fig. V.7b, the effect of location shows a great influence on the temperature of the subzones. Namely, in the cases of Side Walls and Floor exposure, the temperature is elevated. Other boundary conditions (All, Roof and Ground, Roof) is lower by $0.5^{\circ} \mathrm{C}$ in zone mean air temperature.

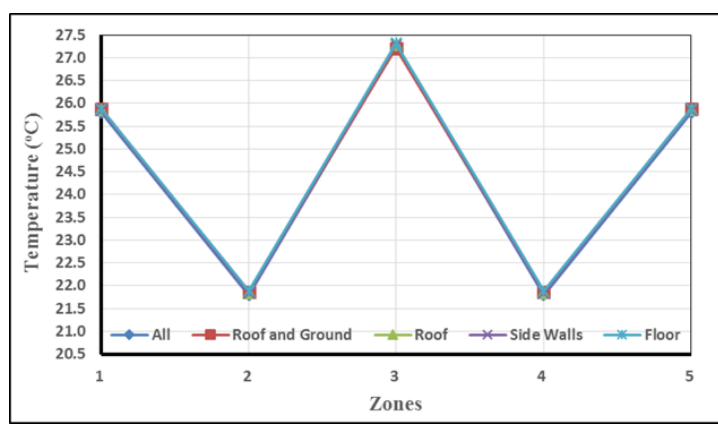

a) Miami

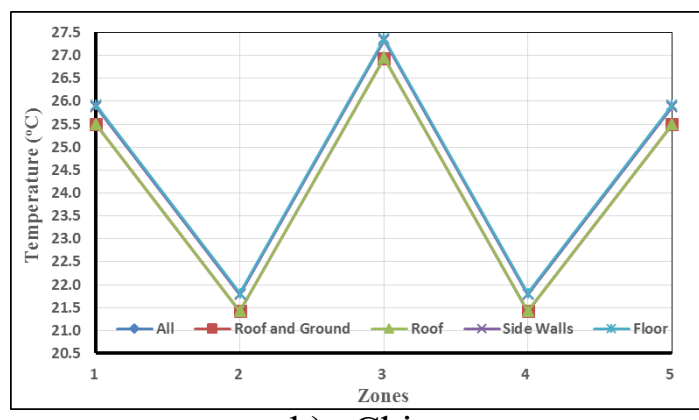

b) Chicago

Fig. V.7 Zone mean air temperature at different zones

Fig. V.8 and Fig. V.9 show the monthly temperature profile at different subzones inside the data center model in Miami and Chicago, respectively. In Fig. V.8, the zone mean air temperature stratification inside the data center model is relatively identical in Miami case, though Side Walls and Floor conditions are quite sensitive in the summer months. This can be seen by a larger bent curve from April to October in these two cases compared with others. The maximum air temperature is in the order of $27.5^{\circ} \mathrm{C}$ (Zone 3 ) and the lowest is $21.5^{\circ} \mathrm{C}$ (Zone $1 \& 5$ ). For the case of Chicago, as seen in Fig. V.9, there is a remarkable difference in winter months as the lines are diverging from the pivot point, namely, the month of July, which clearly indicates the effect of locations on the zone temperature. As the matter of fact, Roof as well as Roof and Ground exposure conditions are easily 
distinguishable from others as the lines are bending over in winter months while other boundary conditions remain quite constant throughout the year.

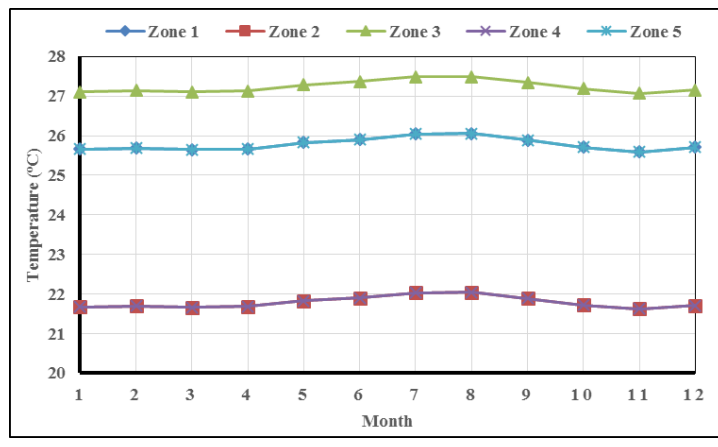

a) All

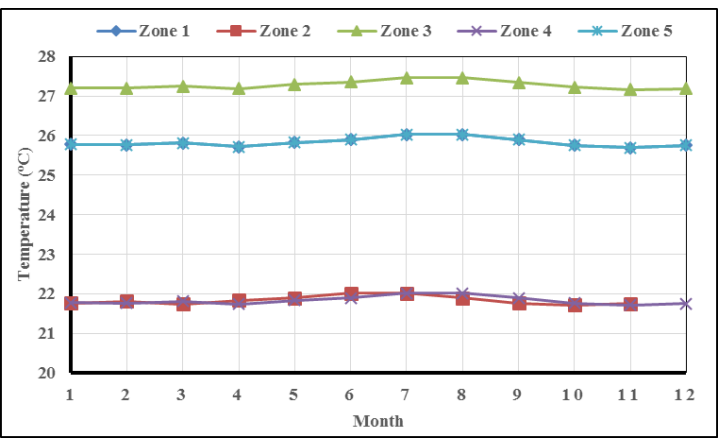

b) Roof and Ground

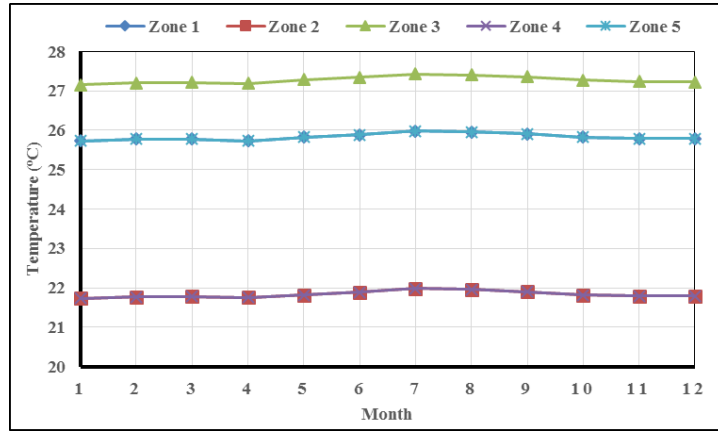

c) Roof

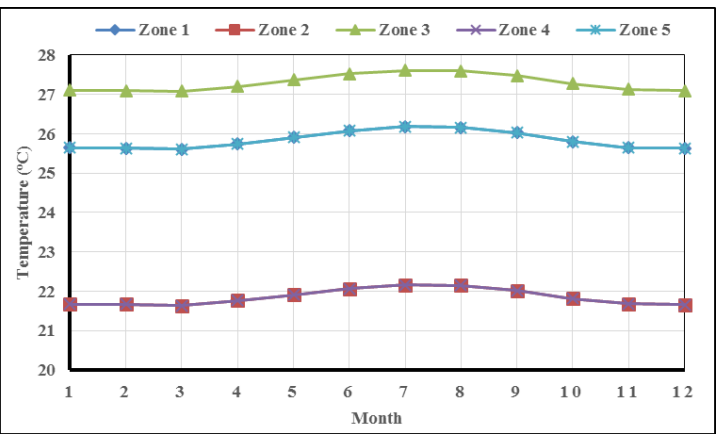

d) Sidewalls

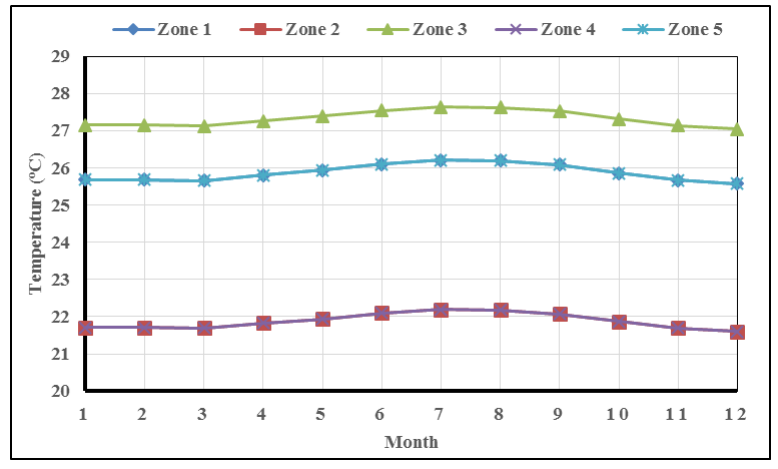

e) Floor 


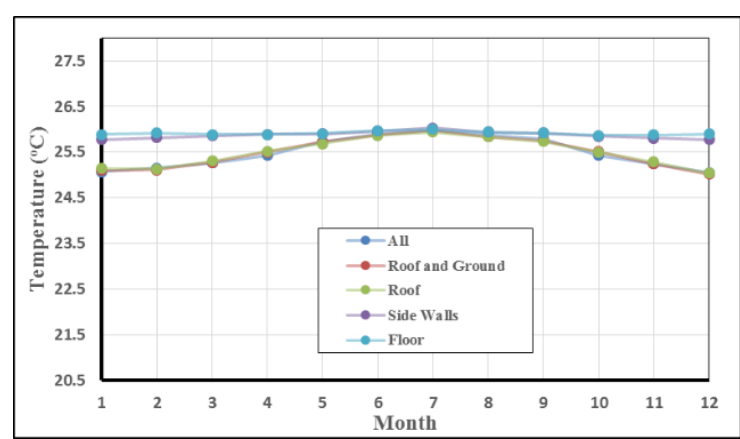

a) Zone 1 (Hot Aisle)

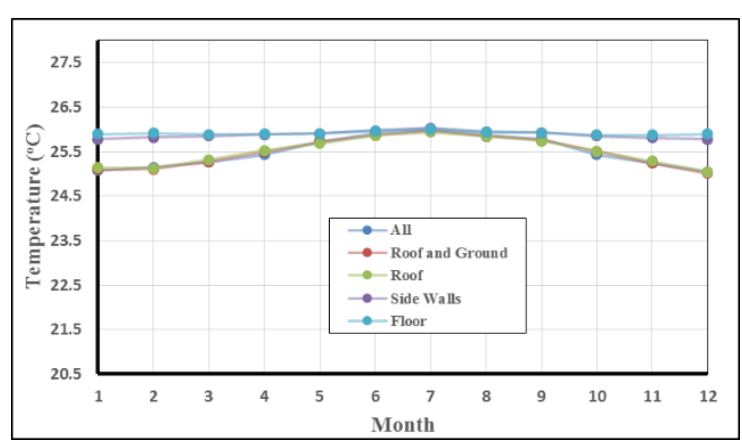

c) Zone 5 (Hot Aisle)

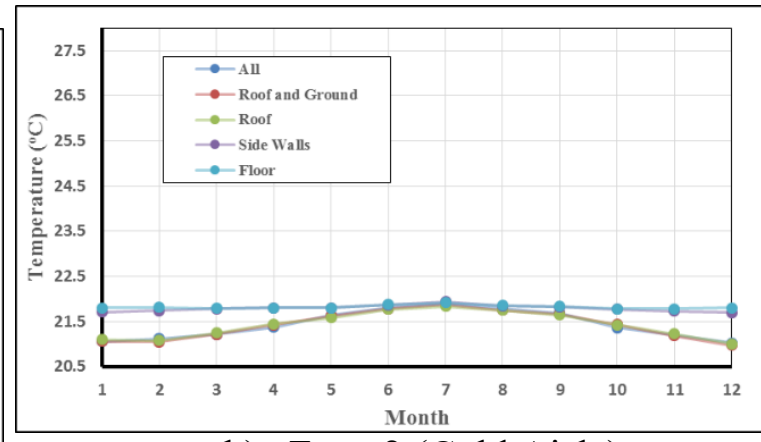

b) Zone 2 (Cold Aisle)

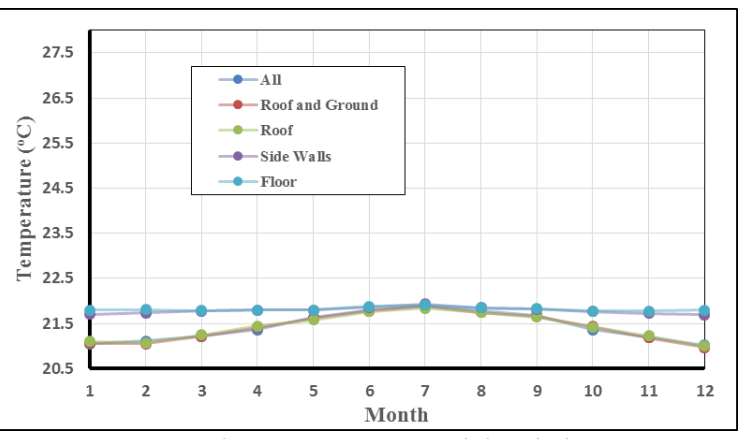

d) Zone 4 (Cold Aisle)

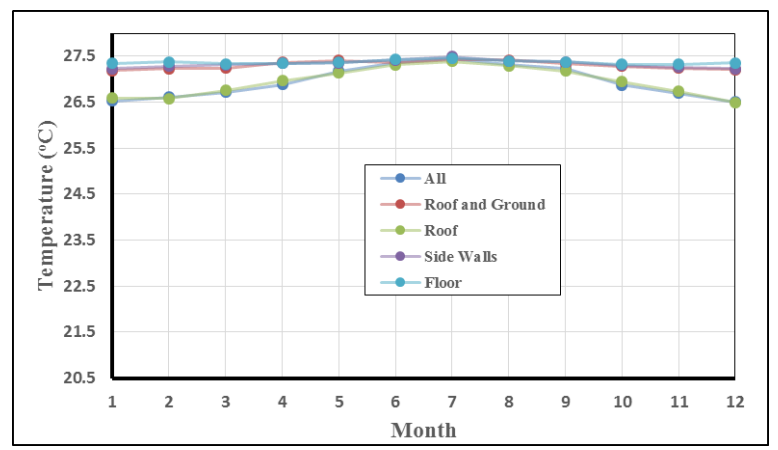

e) Zone 3 (Hot Aisle)

Fig. V.9 Chicago monthly temperature profile at different zones 
Fig. V.10 shows the cooling electricity consumption for winter design day (January 21) in Miami and Chicago, respectively. It is interesting to see that though showing much fluctuation, the lines are comparatively constant throughout the day in both locations. This is understandable since the HVAC system can readily make use of the available chill air from the outside to cool the data center with a less intensive use of cooling electricity.

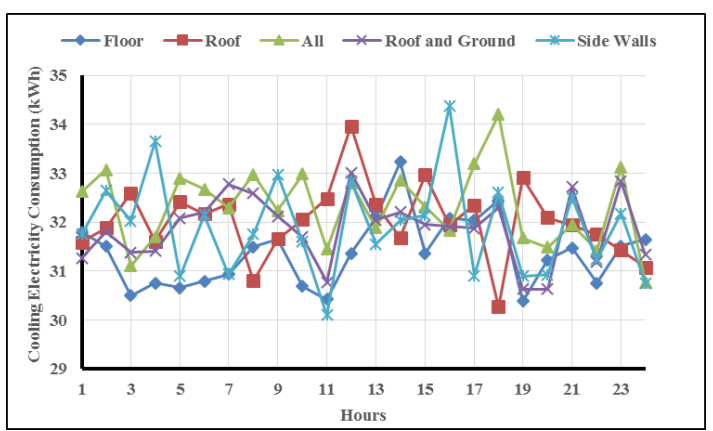

a) Miami

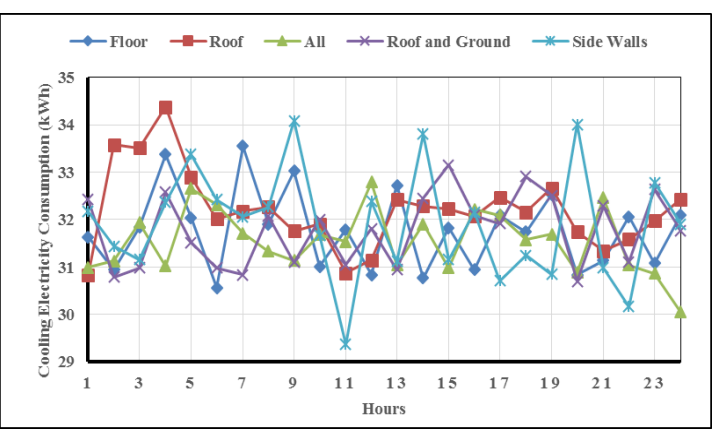

b) Chicago

Fig. V.10 Winter design day for cooling electricity consumption

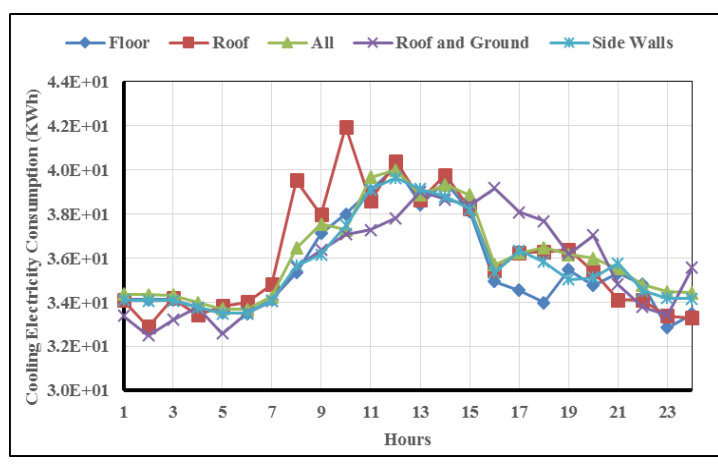

a) Miami

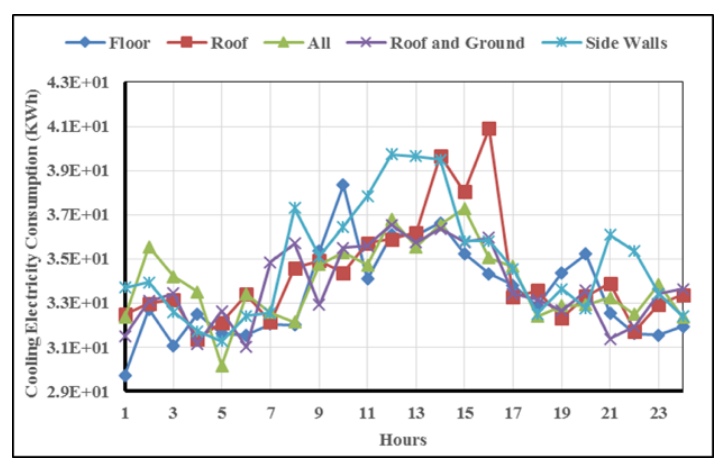

b) Chicago

Fig. V.11 Summer design day for cooling electricity consumption 
Fig. V.11 shows the cooling electricity consumption for summer design day (July 21) in Miami and Chicago, respectively. Evidently, from these two figures, it can be seen that Miami weather tends to give a more stable cooling power consumption as the lines overlapping one another, while there is an apparent fluctuation in cooling power consumption among the various boundary conditions. Comparatively, there is less cooling power consumption in Chicago than in Miami as the lines suggest in both figures.

\subsubsection{Effects of Boundary Conditions}

To further illustrate the influences of boundary conditions, Fig. V.12 shows the annual cooling load at different boundary conditions. As seen on this figure, the effect of surface exposure has a significant control of the annual cooling load. Miami belongs to a very hot and humid climate, so it is obvious that the cooling load is much higher than that of Chicago. The figure demonstrates, in both locations, the Floor exposure condition provides a least amount of annual cooling load. That is, when only the floor of the data center model is connected to the ground, the building requires a lower cooling load from the HVAC system. In addition, Fig. V.12 also shows the Roof exposure condition in Chicago requires most cooling load, while it is the case when All surfaces are exposing to the outside condition in Miami. 


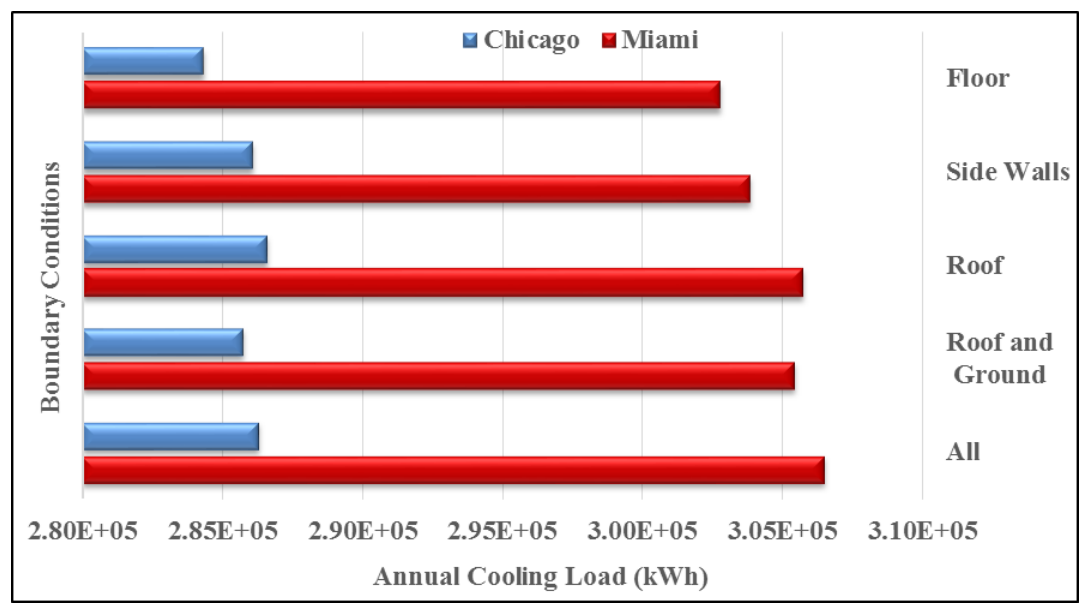

Fig. V.12 Annual cooling load at different boundary conditions

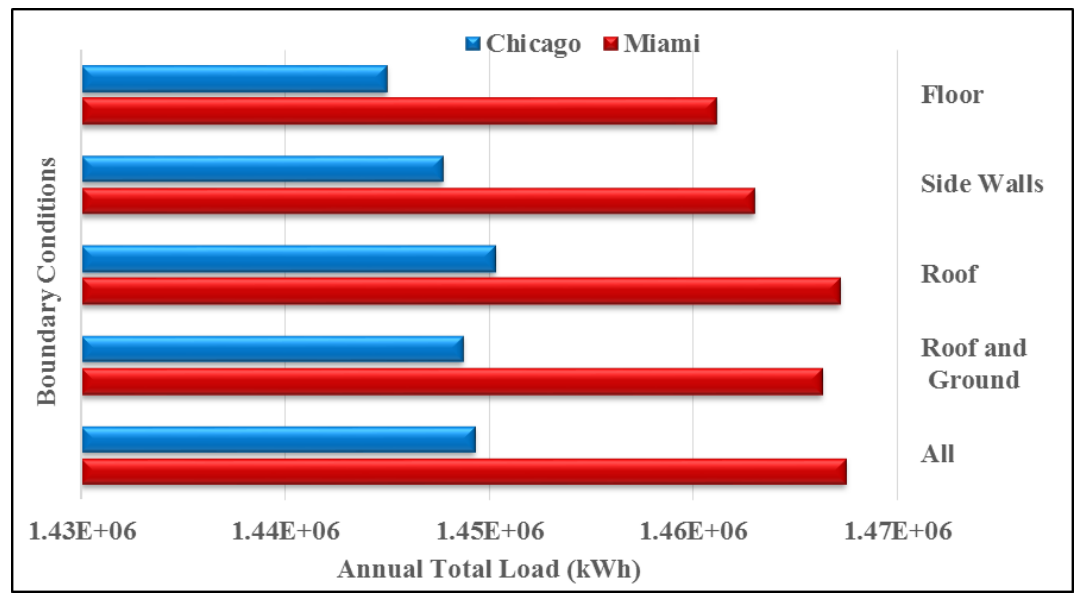

Fig. V.13 Annual total load at different boundary conditions

Fig. V.13 shows the annual total load including fan, cooling, and equipment loads at different boundary conditions. First, Floor exposure conditions in both locations still holds as the case of demanding least load compared to other boundary conditions. Second, Fig. V.13 also shows that the highest annual total energy consumption for Miami happens when 
all surfaces expose to the outside weather, and it is highest for Chicago when only Roof exposes.

Fig. V.7b also shows the effect of boundary condition on zone mean air temperature for Chicago. There is a temperature deviation between two groups of surface exposure. The cases whose Floor and Side Walls are exposing to the outside conditions are prone to produce a higher zone mean air temperature inside the data center compared to other cases. Nevertheless, in the case of Miami (Fig. V.7a), there is no distinction among the boundary conditions. The zone mean air temperature remains the same regardless of the different types of surface exposure. The mild change in Miami's temperature around the year technically accounts for this behavior.

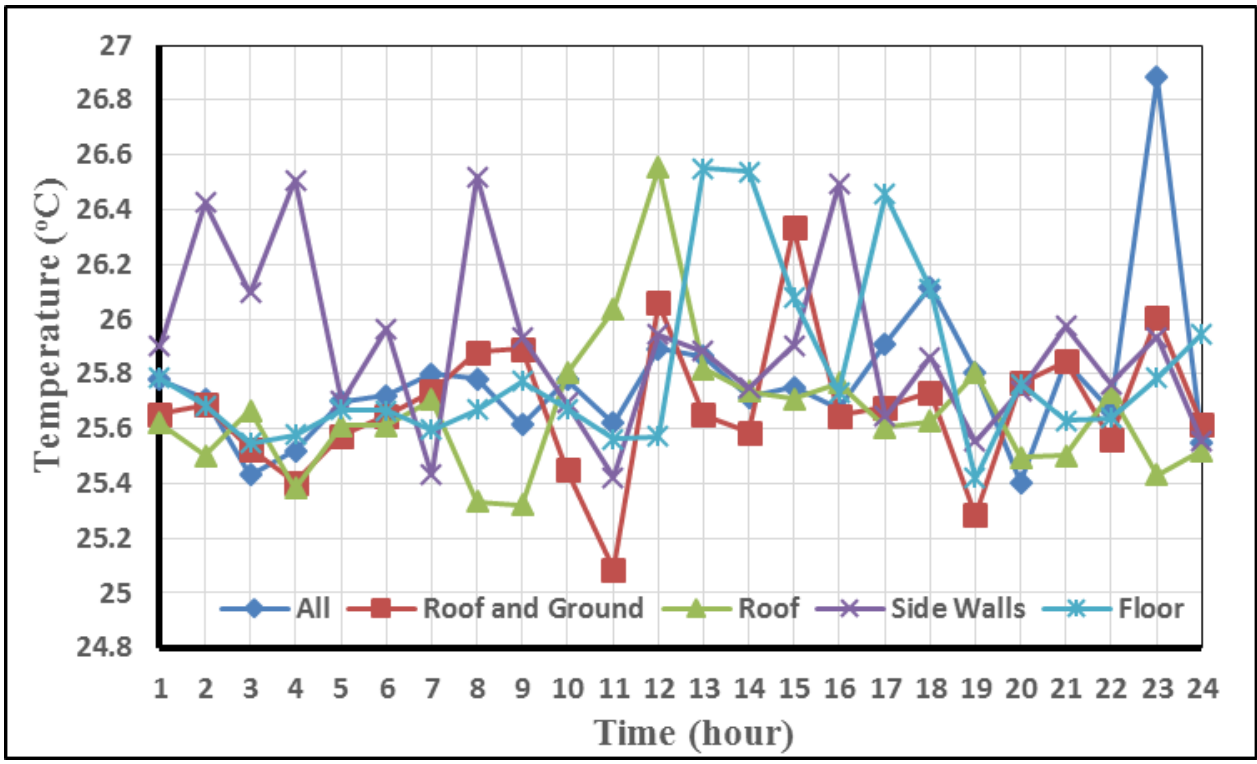

Fig. V.14 Miami winter design day temperature profile 


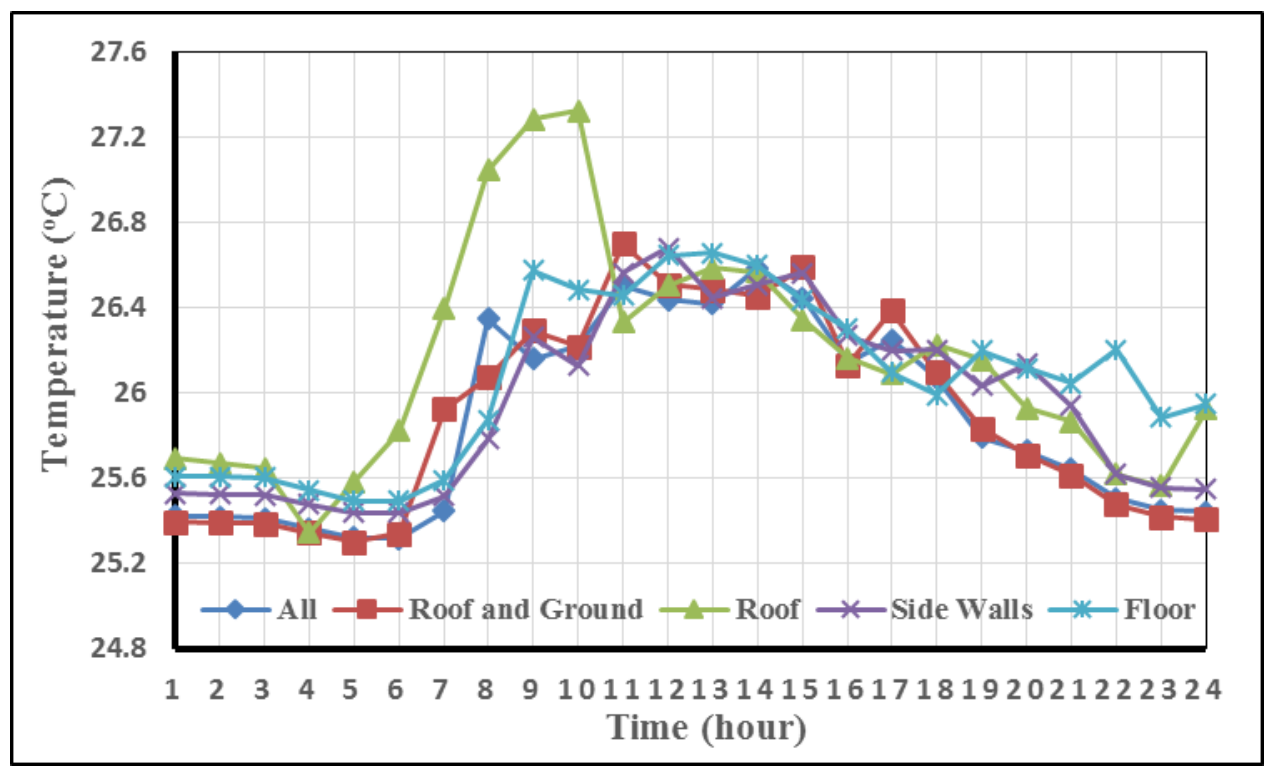

Fig. V.15 Miami summer design day temperature profile

Fig. V.14 and Fig. V.15 show the zone mean air temperature profile for winter and summer design days in Miami. In Fig. V.14, though the lines are wavy, they all alter around a constant temperature line at approximately $25.7^{\circ} \mathrm{C}$ throughout the winter design day. In the same fashion, however, on summer design day as seen in Fig. V.15, the temperature curves are fairly constant during the night hours but start to increase in the afternoon and then settle back on at a constantly lower temperature during the evening hours. There are little to no changes regardless of the presence of various boundary conditions.

\subsubsection{Effects of Supply Temperature}

The effect of supply temperature on the data center energy consumption is shown in Fig. V.16. Although the default temperature (ASHRAE recommended) is $12.8^{\circ} \mathrm{C}$, two more temperature points were chosen both on the left and right hand sides of this default 
temperature. In Fig. V.16, the supply volumetric flow rate was set to be auto-calculated by the software, and the supply temperature was varied. For all boundary conditions, the supply temperature variation shows a consistently parabolic trend. In Fig. V.16a, the cases are separated in three distinct level of energy consumption. The All and the Roof and Ground exposure cases show highest energy consumption. This reflects the typical high temperature of Miami weather, especially in the ground surface and the long hour of solar irradiation. Two cases in the middle range are the Floor and the Roof cases, while the Side Walls condition shows the least energy consumption. Above $12.8^{\circ} \mathrm{C}$, the lines are relatively consistent, while below $12.8^{\circ} \mathrm{C}$, the Floor and the Roof cases are slightly deviated from each other, and the Floor consumes more energy. This is due to the preheated condition when the floor is in contact with the ground surface, thus, the cool air is partially heated by the ground surface temperature.

In Fig. V.16b, the energy consumption of the fan is basically directly proportional to the supply temperature. The higher the supply temperature, the harder the fan has to work to push the cool air through the perforated tiles to maintain a desired temperature inside the data center. The fan load is minimum at $10.8^{\circ} \mathrm{C}$ and maximum at $14.8^{\circ} \mathrm{C}$.

In Fig. V.16c, the PUE for all the cases show a very highly efficient data center since they are all below 1.13, which corresponds to $88.5 \%$ efficiency. The Side Walls is the most efficient case, while the All exposure condition is the least. Clearly, when the supply temperature is lower, the PUE is close to 1 (the ideal case). However, if the supply 
temperature is lower than $10.8^{\circ} \mathrm{C}$, it results in a diverge simulation. Hence, it will not reflect the real-life situation of an HVAC system.

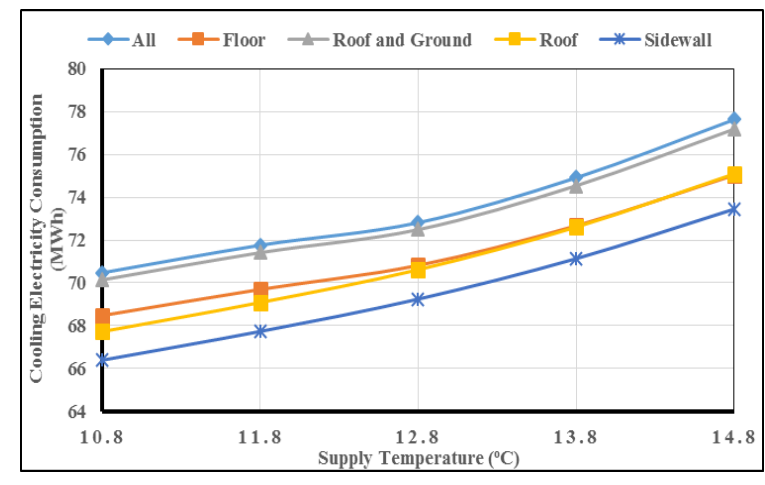

a) Cooling Load

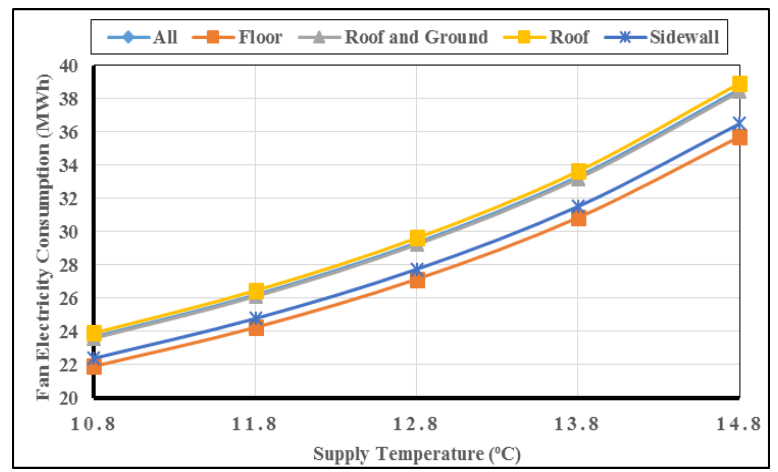

b) Fan Load

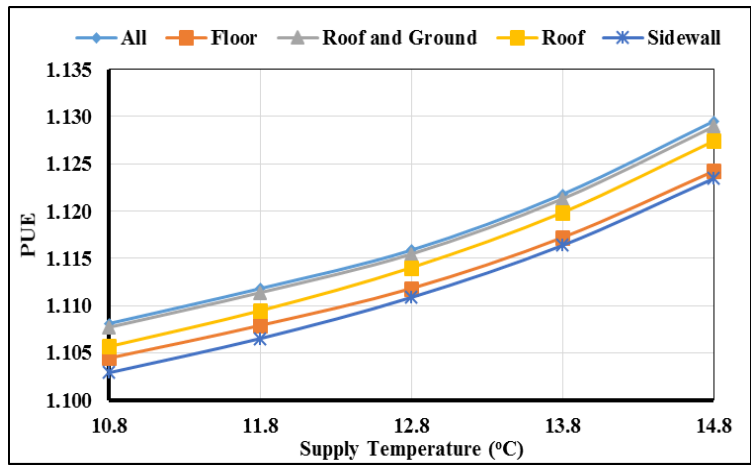

c) Power Use Effectiveness

Fig. V.16 The effect of supply temperature on energy consumption 


\subsubsection{Effects of Volumetric Flow Rate}

Fig. V.17 shows the effect of volumetric flow rate on the data center energy consumption. In this case, the supply temperature was fixed at $12.8^{\circ} \mathrm{C}$, and various volumetric flow rates including auto-size scenario on the supply fan at the raise floor plenum were simulated to seek the optimal energy consumption. In Fig. V.17a, the auto-size data points are represented in green points. Clearly, in the case of auto-size, they tend to scatter around 71-75 MWh with an air flow rate range from 1.1 to $1.5 \mathrm{~m}^{3} / \mathrm{s}$. Since the air flow rate was set to be automatically sizing at both the raised floor plenum end and the system supply fan end, the auto-sizing data are not lying on the solid lines representing their corresponding boundary conditions. In terms of fan cooling electricity consumption, the auto-size results seem to be intensified for the cases of All and Roof boundary conditions and undermined for the case of Floor, Sidewall, and Roof and Ground. For instance, in All boundary condition, with $1.1 \mathrm{~m}^{3} / \mathrm{s}$ of air flow rate, it only gives $73 \mathrm{MWh}$ of energy consumption. Apparently, it is $75 \mathrm{MWh}$ in the case of auto-size condition at the same flow rate. However, if we lower the flow rate to $0.8 \mathrm{~m}^{3} / \mathrm{s}$, energy consumption can be lowered as much as only $65 \mathrm{MWh}$. 


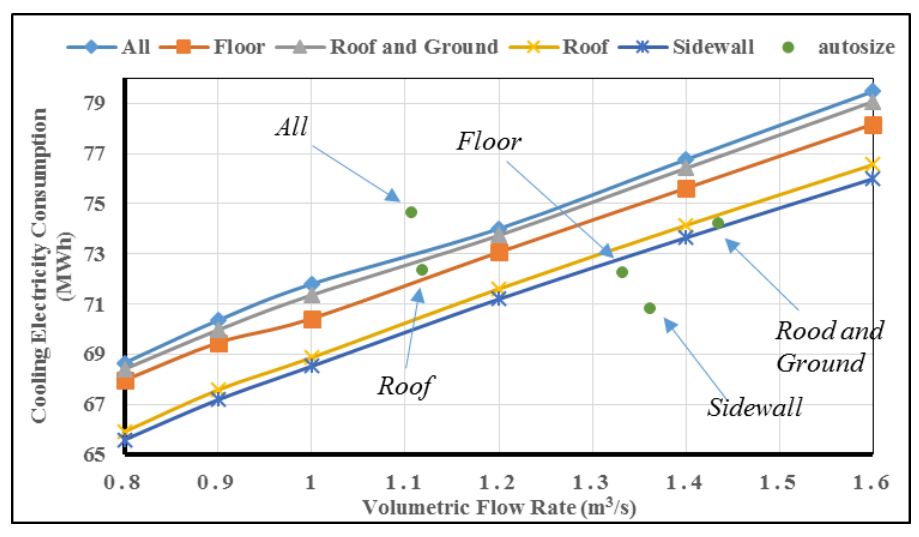

a) Cooling Load

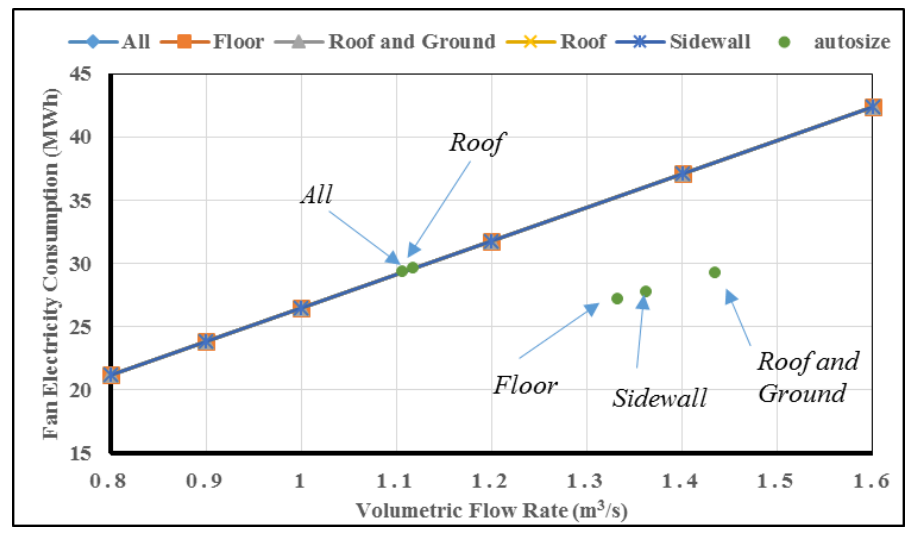

b) Fan Load

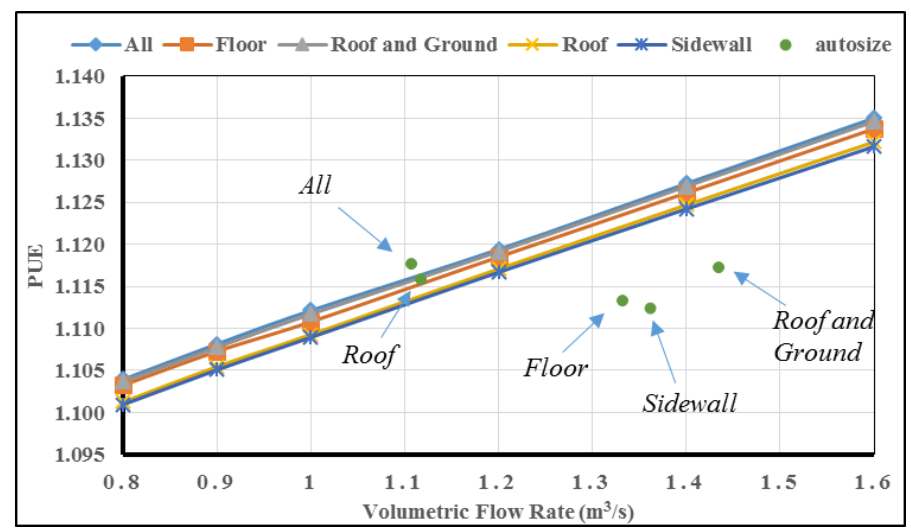

c) Total Energy Consumption

Fig. V.17 The effect of volumetric flow rate on energy consumption 
Fig. V.17b shows the linear relationship of energy consumption and volumetric flow rate. When the air flow rate increases, the energy used in the fan system also increases. The lowest energy consumption is at $0.8 \mathrm{~m}^{3} / \mathrm{s}$ and the highest is at $1.6 \mathrm{~m}^{3} / \mathrm{s}$. Again, we cannot lower the flow rate any further than $0.8 \mathrm{~m}^{3} / \mathrm{s}$ because it will result in an inconsistent data in the simulation process.

Fig. V.17c shows the power use effectiveness (PUE) of different flow rates. All the cases have the PUE less than 1.14. The auto-size cases for all the boundary conditions are relatively constant (1.110-1.115). The cases are very close to one another. At lower air flow rates, the PUE for different boundary conditions is close to 1 . The auto-size scenario is clearly not the case for optimal energy consumption. Rather, the range of $0.8-1.1 \mathrm{~m}^{3} / \mathrm{s}$ gives a better PUE. However, keep in mind that lowering the flow rate temperature will also result in a higher air temperature inside the data center.

\subsection{Remarks}

In the present chapter, a multi-zone modeling strategy was proposed to study the effects of both location and boundary conditions on data center energy and thermal performance. Two typical U.S. climates, Miami (hot and humid) and Chicago (cool and humid), and five boundary conditions were considered. In addition, the effects of both supply temperature and volumetric flow rate conditions on data center energy performance for Miami was also investigated. 
The multi-zone model was verified with CFD and the temperature results are very close to each other compared to single zone approach. Since zone temperature has a direct impact on the energy consumption inside the data center model, the confidence in multi-zone model's energy usage is considered more reliable as compared to single zone model.

The location factor plays an important role to decide whether some of boundary conditions are affecting the cooling electricity consumption throughout the months or not. Miami cases have shown significant differences among the surface exposure conditions, while Chicago cases have almost no effect on the cooling power consumption. In addition, location effect also has a great impact on the zone mean air temperature inside the data center. Miami cases have no changes in subzone temperature among various boundary conditions, whereas Chicago cases show clear temperature distinction on the surface exposure conditions.

The boundary condition is a useful indicator to recognize whether a certain type of surface exposure would result in the least cooling electricity consumption. It is found that when only Floor is exposed, the data center model consumes the least amount of cooling power in both locations. However, in terms of maximum amount of cooling electricity consumption, it is the Roof exposure condition that requires most cooling power in Miami, while it is the All surface exposure condition in Chicago. 
It has been found that the effect of supply temperature has significant effect on the energy consumption. ASHRAE recommended supply temperature for general buildings were not the case in this study. Rather, with a supply temperature of $10.8^{\circ} \mathrm{C}$, the PUE is very close to 1 which also means a very efficient data center.

The effect of volumetric flow rate also plays an important role on the data center energy consumption. It is found that in the supply air flow rate range of $0.8-1.1 \mathrm{~m}^{3} / \mathrm{s}$, the PUE is closest to 1 . 
CHAPTER VI CONCLUSION 


\subsection{Summary}

The literature review in chapter 1 shows that common practices in using CFD for thermal management of data centers is quite popular. However, these CFD methods normally require an extensive amount of run time and computational resource. For hyperscale data centers, this issue is even magnified. In fact, CFD method is only proven useful for predicting both thermal and velocity profiles inside a data center in offline studies. For real-time thermal monitoring or online studies which require constant input/output results, the method becomes problematic. The purpose of this research is to explore the alternative methods to solve the two posed challenges, i.e., run time and accuracy. By adopting novel methods such as reduced order modeling, e.g., proper orthogonal decomposition, and metamodel approximation technique, e.g., response surface methodology, the research aims to reduce the modeling time while keeping a reasonable accuracy for data center studies. The tradeoffs between these two quantities are carefully investigated and valid conclusions are drawn. Besides, other studies related to data centers such tile models, turbulence models, hybrid turbulence model, and optimizations also discussed in detail to add values to for data center research.

In the first part of chapter 2, by acknowledging the two regions in a data center, namely, the "non-viscous domain" and "viscous domain," a hybrid turbulence combination is introduced to reduce the computational effort in simulation. The "non-viscous domain" is referred to the region where there is insignificant and less turbulent flow characteristics such as the region far away from the active tile region. In these low to non-viscous regions, 
the zero-equation model is used. The zero-equation model shows advantages in indoor airflow modeling because of its fast running time due to substantial grid size reduction, compared to the $k-\varepsilon$ models. In the viscous or more active regions such around the server racks and floor tiles, the standard $k-\varepsilon$ turbulence model is used to ensure the accuracy of the modeling results. This coupling method tries to preserve the accuracy in more active flow regions while reduce the computing effort in less active flow areas.

In the second part of chapter 2, a comprehensive evaluation of floor tile and turbulence models is presented. In this section, a detailed validation study of ten turbulence models is investigated. Also, seven tile models are studied and compared. The rack model at three tile-to-server airflow ratios, $20 \%, 60 \%$, and $100 \%$, are assessed. Discussion on the pros and cons within the available flow conditions, as well as suggestions based on computing time and accuracy of each turbulence model are also provided. By quantifying the root mean square error (RMSE) and assessing the computing time of each turbulence model at various flow conditions, an overall performance index is proposed for each turbulence and title model relative to the baseline model. Top tile and turbulence model combinations are recommended based on the targeting criteria in regard to the tradeoff between computing time and accuracy.

In chapter 3 , thermal modeling of data center is carried out using a proper orthogonal decomposition method based on CFD data. A well-representative model of a real data center having three hot aisles and two cold aisles, and four CRAC units placed at four opposite walls is studied. The effects in the number of parameters used for the POD basis 
function on the accuracy of the thermal modeling of data centers are investigated. In addition, the main focus is on the number of input parameter used to construct the POD basis function. In the current study, POD method is applied to obtain the temperature profile of a data center model using three input parameters, i.e., rack heat load, mass flow rate, and inlet temperature. An interpolation technique of constructing 3-D picture of the temperature profiles using 2-D POD simulation is introduced for the first time in data center modeling at various input conditions. Effects of various combinations of three design parameters previously mentioned are also investigated. Sensitivity analysis of each design parameter on the overall temperature is done individually to assess the fidelity of the POD model as compared to the CFD model.

In chapter 4 , response surface methodology is studied for the first time in thermal modeling and optimization studies for data centers. Due to its rapid running time, RSM can be used to accommodate the optimization of multiple design parameters where thousands of virtual design scenarios need to be generated. In addition, RSM can also be applied to reconstruct the airflow and temperature fields quickly, which is potentially useful for real-time thermal monitoring of data centers. Throughout the chapter, four applications of RSM for data centers are introduced. The first application focuses on finding an interpolation function that can be used to reconstruct the thermal profile within a data center model. Three different percentages of the original CFD data points $(5 \%, 10 \%$, and $20 \%)$ are compared with each other for training the response surface model in to get the overall temperature profile of a data center model. In second application, RSM model is applied with the goal of minimizing both the temperature difference of across the server racks and the maximum 
temperature within a data center model. The optimization process allows to seek the best design parameters that satisfy both of the said objectives. In the third application, the RSM approach is demonstrated with the objective of seeking the best tile porosity arrangement that can create a uniform airflow distribution through perforated tiles. In the fourth application, a prescribed airflow distribution of choice is obtained through the inverse design optimization, and three different scenarios including linear, parabolic, and sinusoidal tile airflow distributions are optimized with success.

In chapter 5, although building energy simulation can be used to estimate annual energy consumption of any building, its application in data centers has been very limited in the open literatures. Previous studies have only adopted the well mixed single-zone approach, without considering the existence of hot and cold aisles in the data center. Considering the importance of the hot and cold isles in thermal management, a multi-zone modeling approach that resolves the existence of the hot/cold aisles is proposed. In addition, data center thermal and energy performance under different boundary conditions are also investigated systematically in this chapter. The objectives are to first develop a more reasonable simulation model for data center in order to resolve the hot and cold aisles, and then apply the developed model to perform systematic studies of data center's thermal and energy performance under different conditions. A parametric study using the BES code, EnergyPlus, is conducted to study the effects of five different surface boundary conditions (surface exposures) on the cooling electricity consumption and the zone mean air temperature in the data center. The studies are carried out for two typical U.S. climates: 
hot and humid (Miami, FL), and cool and humid (Chicago, IL). In addition, the effects of both supply temperature and volumetric flow rate of the supply side are also investigated.

\subsection{Conclusions}

Throughout the early dissertation work, coupled methods using zero-equation and standard $k-\varepsilon$ shows proven advantages over the common CFD practice of using one single viscous domain on with two-equation models such as standard $k$-E. A comparison between the two methods shows that the former method consumes half of the computing time compared to the latter within the same data center model. Next, regarding the comprehensives study of various RANS turbulence models and tile models in three typical flows (i.e., low, medium, high), a proposed composite performance index based on three modeling purposes (i.e., accuracy-targeting, time-targeting, and well-balanced models) is identified for data center modeling. Although some turbulence models are more advantageous compared to others in each modeling purpose, overall, it is found that the use of zero-equation turbulence model combined with either body force or modified body force tile model suggests reasonably good outcomes for data center modeling.

In developing faster methods for data center modeling, both POD and RSM have good potentials in reducing the computing time while keeping reasonable accuracy for data center modeling. Both of these methods, though different in approaches (reduced order vs. approximation), can successfully reconstruct the temperature profiles within a data center 
model. This is especially advantageous for online study of data centers. Further applications of RSM show usefulness in design input optimization on temperature differences across server racks and maximum temperature within a data center. The time reduction is superior compared to the traditional CFD method. It only takes 7 days for the entire process with RSM, whereas 379 days is required for CFD. Finally, the successful optimizations of both tile airflow uniformity and inverse designs of various tile airflows shows that RSM's capability in assisting best practice floor tile selection for an energyefficient data center.

Finally, in energy modeling for data centers, the proposed multi-zone model is found to be more effective compared to the commonly used single zone model. For two specific locations investigated, i.e., Miami and Chicago, the energy consumption would have been saved $16 \%$ in Miami and 14\% in Chicago if the multi-zone model was to be used over the single-zone model. In addition, the average zone temperature difference for the multi-zone model is much improved (7\%) compared to single-zone model (19\%). Location factor plays an important role in deciding whether some of boundary conditions are affecting the cooling electricity consumption on a monthly basis. In addition, the effect of supply temperature and volumetric flow rate have significant effects on the energy consumption. Within the goal of reaching PUE close to unity, a supply temperature of $10.8^{\circ} \mathrm{C}$ and a flow rate range of $0.8-1.1 \mathrm{~m}^{3} / \mathrm{s}$ are suggested for the data center investigated. 


\subsection{Future Work}

Although the hybrid turbulence model significantly reduces the computing time in half, it faces the problem of geometry-specific or application-specific. The current algorithm focuses on the detection of non-viscous and viscous regions based primarily on the vorticity magnitude level for the data center model studied; however, a more robust algorithm needs to be in place to better identify the non-viscous and viscous regions for any geometries.

As for the POD method, the current study focuses on CFD-based POD method using snapshots to construct 3-D temperature profile from 2D. In reality, sensor-based POD can be alternative used to construct the temperature field. Temperature sensors should be placed in a steady surface and sweeping through the entire data center for temperature input that is then analyzed by POD method. If successfully applied, it is particularly useful for detect hotspots in real-time for temperature control of data centers.

The POD and RSM methods are serving as the same purpose for time reduction and accuracy preservation. However, the comparison of these two methods have not been analyzed at the same time in this thesis work. Future studies should include the comparison in detail on the advantages and disadvantages of both methods. 


\section{LIST OF REFERENCES}

[1] Abdelmaksoud, W. A., Dang, T. Q., Khalifa, H. E., \& Schmidt, R. R. (2013). Improved computational fluid dynamics model for open-aisle air-cooled data center simulations. Journal of Electronic Packaging, 135(3), 030901.

[2] Abdelmaksoud, W. A., Khalifa, H. E., Dang, T. Q., Elhadidi, B., Schmidt, R. R., \& Iyengar, M. (2010, June). Experimental and computational study of perforated floor tile in data centers. In Thermal and Thermomechanical Phenomena in Electronic Systems (ITherm), 2010 12th IEEE Intersociety Conference on (pp. 1-10). IEEE.

[3] Abdelmaksoud, W. A., Khalifa, H. E., Dang, T. Q., Schmidt, R. R., \& Iyengar, M. (2010, June). Improved CFD modeling of a small data center test cell. In 2010 12th IEEE Intersociety Conference on Thermal and Thermomechanical Phenomena in Electronic Systems (pp. 1-9).

[4] Ahmad, F., \& Vijaykumar, T. N. (2010, March). Joint optimization of idle and cooling power in data centers while maintaining response time. In ACM Sigplan Notices (Vol. 45, No. 3, pp. 243-256). ACM.

[5] American Society of Heating, Refrigerating and Air-Conditioning Engineers. (2012). Datacom Equipment Power Trends and Cooling Applications.

[6] ANSYS Inc., Fluent. https://www.ansys.com/products/fluids/ansys-fluent

[7] Arghode, V. K., \& Joshi, Y. (2014). Room level modeling of air flow in a contained data center aisle. Journal of Electronic Packaging, 136(1), 011011.

[8] Arghode, V. K., \& Joshi, Y. (2014, May). Rapid modeling of air flow through perforated tiles in a raised floor data center. In Thermal and Thermomechanical Phenomena in Electronic Systems (ITherm), 2014 IEEE Intersociety Conference on (pp. 1354-1365). IEEE.

[9] Arghode, V. K., Kumar, P., Joshi, Y., Weiss, T., \& Meyer, G. (2013). Rack level modeling of air flow through perforated tile in a data center. Journal of Electronic Packaging, 135(3), 030902.

[10] Box, G. E. \& Wilson, K. B. (1951). On the experimental attainment of optimum conditions. Journal of the Royal Statistical Society: Series B (Methodological), 13(1), pp. $1-38$.

[11] Chatterjee, A. (2000). An introduction to the proper orthogonal decomposition. Current science,pp. 808-817. 
[12] Chen, H., Reuss, D. L., \& Sick, V. (2012). On the use and interpretation of proper orthogonal decomposition of in-cylinder engine flows. Measurement Science and Technology, 23(8), 085302.

[13] Chen, Q., \& Xu, W. (1998). A zero-equation turbulence model for indoor airflow simulation. Energy and buildings, 28(2), 137-144.

[14] Cho, J., \& Kim, B. S. (2011). Evaluation of air management system's thermal performance for superior cooling efficiency in high-density data centers. Energy and buildings, 43(9), 2145-2155.

[15] Chowdhury, A.A., Rasul, M.G., and Khan, M.M. Analysis of building systems performance through integrated computation fluid dynamics technique. Proceedings of the $13^{\text {th }}$ Asian Congress of Fluid Dynamics, pp. 625-628, 2010.

[16] Cizmas, P. G., Palacios, A., O'Brien, T., \& Syamlal, M. (2003). Properorthogonal decomposition of spatio-temporal patterns in fluidized beds. Chemical engineering science, 58(19), 4417-4427.

[17] Colaço, M. J. \& Dulikravich, G. S. (2008). A hybrid RBF based method for highly multidimensional response surfaces using scarce data sets. In 12th AIAA/ISSMO Multidisciplinary Analysis and Optimization Conference, Victoria, British Columbia, Canada, pp. 5892.

[18] Colaço, M. J. \& Dulikravich, G. S. (2009). A survey of basic deterministic, heuristic and hybrid methods for single-objective optimization and response surface generation. Thermal Measurements and Inverse Techniques, 1, pp. 355-405.

[19] Colaço, M. J., Dulikravich, G. S. \& Sahoo, D. (2008). A response surface method-based hybrid optimizer. Inverse Problems in Science and Engineering, 16(6), pp. 717-741.

[20] Dulikravich, G. S. \& Colaco, M. J. (2015). Hybrid optimization algorithms and hybrid response surfaces. Chapter 2 in Advances in Evolutionary and Deterministic Methods for Design, Optimization and Control in Engineering and Sciences (eds.: D. Greiner, B. Galván, J. Periaux, N. Gauger, K. Giannakoglou, G. Winter), Computational Methods in Applied Sciences Series, Springer Verlag, pp. 19-47.

[21] Durbin, P. A. (1995). Separated flow computations with the k-epsilon-v-squared model. AIAA journal, 33(4), 659-664.

[22] Egorov-Yegorov, I. N. \& Dulikravich, G. S. (2005). Chemical composition design of superalloys for maximum stress, temperature, and time-to-rupture using self-adapting response surface optimization. Materials and Manufacturing Processes, 20(3), pp. 569590. 
[23] EnergyPlus Engineering Reference, EnergyPlus 7.2, 2012, www.energyplus.gov.

[24] Escudier, M. P. (1965). The distribution of the mixing length in turbulent flows near walls. Mechanical Engineering Department.

[25] ESTECO S.R.L., modeFrontier ver. 450., Response surface training manual. http://www.estecom.com/

[26] Fulpagare, Y., Mahamuni, G., \& Bhargav, A. (2015). Effect of plenum chamber obstructions on data center performance. Applied Thermal Engineering, (80), pp. 187195.

[27] Gibson, M. M., \& Launder, B. E. (1978). Ground effects on pressure fluctuations in the atmospheric boundary layer. Journal of Fluid Mechanics, 86(3), 491-511.

[28] Google Sketchup, www.sketchup.com

[29] Greenberg, S., Mills, E., Tschudi, B., \& Rumsey, P. (2006). Myatt. Best practices for data centers: results from benchmarking 22 datacenters. In Proceedings of the 2006 ACEEE Summer Study on Energy Efficiency in Buildings (Vol. 16).

[30] Hardy, R. L. (1971) Multiquadric equations of topography and other irregular surfaces. Journal of Geophysics Res., Vol. 176, pp. 1905-1915.

[31] He, J., Sætrom, J., \& Durlofsky, L. J. (2011). Enhanced linearized reduced-order models for subsurface flow simulation. Journal of Computational Physics, 230(23), 83138341.

[32] Healey, C. M., VanGilder, J. W., Sheffer, Z. R., \& Zhang, X. S. (2011, January). Potential-flow modeling for data center applications. In ASME 2011 Pacific Rim Technical Conference and Exhibition on Packaging and Integration of Electronic and Photonic Systems (pp. 527-534).

[33] Hong, T., Sartor, D., Mathew, P., \& Yazdanian, M. (2008). Comparisons of HVAC simulations between EnergyPlus and DOE-2.2 for data centers (No. LBNL1138E). Lawrence Berkeley National Lab.(LBNL), Berkeley, CA (United States).

[34] https://www.theseverngroup.com/data-center-hot-aisle-cold-aisle-layout/. Accessed 04.23.2019

[35] Hu, W., Enying, L. \& Yao, L. (2008) Optimization of sheet metal forming processes by adaptive response surface based on intelligent sampling method. Journal of Materials Processing Technology 197 (1-3) pp. 77- 88.

[36] Iyengar, M., Schmidt, R. R., Hamann, H., \& VanGilder, J. (2007, January). Comparison between numerical and experimental temperature distributions in a small 
data center test cell. In ASME 2007 InterPACK Conference collocated with the ASME/JSME 2007 Thermal Engineering Heat Transfer Summer Conference (pp. 819826).

[37] Jin, M., Zuo, W., \& Chen, Q. (2012). Improvements of fast fluid dynamics for simulating air flow in buildings. Numerical Heat Transfer, Part B: Fundamentals, 62(6), 419-438.

[38] Jin, R., Chen, W. and Simpson, T.W. (2000) Comparative studies of metamodeling techniques under multiple modeling criteria. In Proceedings of the 8th AIAA/USAF/NASA/ISSMO Multidisciplinary Analysis \& Optimization Symposium, AIAA 2000-4801, Long Beach, CA, 6-8 September 2000.

[39] Kansa, E. J., (1990) Multiquadrics - a scattered data approximation scheme with applications to computational fluid dynamics - ii: solutions to parabolic, hyperbolic and elliptic partial differential equations. Comput. Math. Appl., Vol. 19, pp. 149-161.

[40] Kerschen, G., Golinval, J.C., Vakakis, A.F., \& Bergman, L.A. (2005). The method of proper orthogonal decomposition for dynamical characterization and order reduction of mechanical systems: an overview, Nonlinear Dyn. (41)147-169.

[41] Kumar, P., \& Joshi, Y. Experimental investigations on the effect of perforated tile air jet velocity on server air distribution in a high-density data center. In Thermal and Thermomechanical Phenomena in Electronic Systems (ITherm), 2010 12th IEEE Intersociety Conference on (pp. 1-7). IEEE.

[42] Lang, Y., Zitney, S. E., \& Biegler, L. T. (2011). Optimization of IGCC processes with reduced order CFD models. Computers \& Chemical Engineering, 35(9), 1705-1717.

[43] Launder, B. E., \& Sharma, B. I. (1974). Application of the energy-dissipation model of turbulence to the calculation of flow near a spinning disc. Letters in heat and mass transfer, 1(2), 131-137.

[44] Launder, B.E., Spalding, D.B., The numerical computation of turbulent flows. Computer Methods in Applied Mechanics and Engineering, 3(2), pp. 269-289, 1974.

[45] Lettieri, D. J., Toulouse, M. M., Bash, C. E., Shah, A. J., \& Carey, V. P. (2013). Computational and experimental validation of a vortex-superposition-based buoyancy approximation for the COMPACT code in data centers. Journal of Electronic Packaging, 135(3), 030903.

[46] Li, K., Xue, W., Xu, C., Su, H. Optimization of ventilation system operation in office environment using POD model reduction and genetic algorithm. Energy and Building 67 (2013), pp. $34-43$. 
[47] Menter, F. R. (1994). Two-equation eddy-viscosity turbulence models for engineering applications. AIAA journal, 32(8), 1598-1605.

[48] Moore, J. D., Chase, J. S., Ranganathan, P., \& Sharma, R. K. (2005, April). Making scheduling" cool": temperature-aware workload placement in data centers. In USENIX annual technical conference, General Track (pp. 61-75).

[49] Moore, J., Chase, J. S., \& Ranganathan, P. (2006, June). Weatherman: Automated, online and predictive thermal mapping and management for data centers. In 2006 IEEE international conference on Autonomic Computing (pp. 155-164). IEEE.

[50] Moral, R. \& Dulikravich, G. S. (2008). A hybridized self-organizing response surface methodology. In 12th AIAA/ISSMO Multidisciplinary Analysis and Optimization Conference, Victoria, British Columbia, Canada, pp. 5891.

[51] Nielsen, P. V. (1990). Specification of a two-dimensional test case. Aalborg, Denmark: Aalborg University.

[52] Pan, Y., Yin, R., \& Huang, Z. (2008). Energy modeling of two office buildings with data center for green building design. Energy and Buildings, 40(7), 1145-1152.

[53] Pan, Y., Yin, R.X., Huang, Z. (2008). Energy modeling of two office buildings with data center for green building design. Energy and Building 40, pp. 1145-1152.

[54] Patel, C. D., Bash, C. E., Belady, C., Stahl, L., \& Sullivan, D. (2001, July). Computational fluid dynamics modeling of high compute density data centers to assure system inlet air specifications. In Proceedings of IPACK (Vol. 1, pp. 8-13).

[55] Phan, L., \& Lin, C. X. (2016) November). Multi-objective optimization of a data center modeling using response surface. In ASME 2016 International Mechanical Engineering Congress and Exposition, Phoenix, AZ, pp. V008T10A044-V008T10A044.

[56] Phan, L., Telusma, M., \& Lin, C. X. (2015) Data center modeling using response surface with multiple-parameters approach. ASME 2015 International Mechanical Engineering Congress \& Exposition, Houston, TX, USA, November 13 - 19.

[57] Rodríguez, T., and Diego, I. 2006. Computational fluid dynamics (CFD) use in the simulation of the death end ventilation in tunnels and galleries. WIT Transactions on Engineering Sciences 52.

[58] Samadiani, E., \& Joshi, Y., "Multi-parameter Model Reduction in Multi-scale Convection Systems," International Journal of Heat and Mass Transfer, Vol. 53 (2010), pp. $2193-2205$. 
[59] Samadiani, E., \& Joshi, Y., "Proper Orthogonal Decomposition for Reduced Order Thermal Modeling of Air-Cooled Data Centers," Journal of Heat Transfer, Vol. 132 (2010), pp. $1-14$.

[60] Samadiani, E., \& Joshi, Y., "Reduced Order Thermal Modeling of Data Centers via Proper Orthogonal Decomposition: A Review," Int'1 Journal of Numerical Methods for Heat \& Fluid Flow, Vol. 20, No. 5 (2010), pp. 529 - 550.

[61] Sharma, R. K., Bash, C. E., Patel, C. D., Friedrich, R. J., \& Chase, J. S. (2005). Balance of power: Dynamic thermal management for internet data centers. IEEE Internet Computing, 9(1), 42-49.

[62] Sharma, R., Bash, C., \& Patel, C. (2002, June). Dimensionless parameters for evaluation of thermal design and performance of large-scale data centers. In 8th AIAA/ASME Joint Thermophysics and Heat Transfer Conference (p. 3091).

[63] Shehabi, A., Smith, S., Sartor, D., Brown, R., Herrlin, M., Koomey, J., ... \& Lintner, W. (2016). United states data center energy usage report.

[64] ShihT, H., LiouW, W., \& Shabbir, A. (1995). A New Eddy Viscosity Model for High Reynolds Number Turbulent flows. Journal of Computational Fluids, 24(3), 227238.

[65] Sirovich, L. (1987). Turbulence and the dynamics of coherent structures. I. Coherent structures. Quarterly of applied mathematics, 45(3), 561-571.

[66] Sobol, I.M. (1976) Uniformly distributed sequences with an additional uniform property. USSR Computational Mathematics and Mathematical Physics. (16), pp. 236242.

[67] Reddy, S. R., Freno, B. A., Cizmas, P. G., Gokaltun, S., McDaniel, D., \& Dulikravich, G. S. (2017). Constrained reduced-order models based on proper orthogonal decomposition. Computer Methods in Applied Mechanics and Engineering, 321, 18-34.

[68] Spalart, P., \& Allmaras, S. (1992, January). A one-equation turbulence model for aerodynamic flows. In 30th aerospace sciences meeting and exhibit (p. 439).

[69] Toulouse, M. M., Doljac, G., Carey, V. P., \& Bash, C. (2009, January). Exploration of a potential-flow-based compact model of air-flow transport in data centers. In ASME 2009 international mechanical engineering congress and exposition (pp. 41-50).

[70] VanGilder, J. W., \& Schmidt, R. R. (2005, January). Airflow uniformity through perforated tiles in a raised-floor data center. In ASME 2005 Pacific Rim Technical Conference and Exhibition on Integration and Packaging of MEMS, NEMS, and Electronic Systems collocated with the ASME 2005 Heat Transfer Summer Conference, San Francisco, CA, pp. 493-501. 
[71] VanGilder, J. W., Pardey, Z. M., Bemis, P., \& Plamondon, D. W. (2016, May). Compact modeling of data center raised-floor-plenum stanchions: Pressure drop through sparse tube bundles. In Thermal and Thermomechanical Phenomena in Electronic Systems (ITherm), 2016 15th IEEE Intersociety Conference on (pp. 1148-1155). IEEE. [72] VanGilder, J. W., Sheffer, Z. R., Zhang, X. S., \& Healey, C. M. (2011). Potential Flow Model for Predicting Perforated Tile Airflow in Data Centers. ASHRAE Transactions, 117(2).

[73] VanGilder, J. W., Zhang, X. S., \& Healey, C. M. (2013, July). Data center airflow prediction with an enhanced potential flow model. In ASME 2013 International Technical Conference and Exhibition on Packaging and Integration of Electronic and Photonic Microsystems (pp. V002T09A005-V002T09A005).

[74] Wang, G. G. (2003) Adaptive response surface method using inherited latin hypercube design points. ASME J. Mech. Des., 12 5, pp. 210-220.

[75] Wang, H., \& Zhai, Z. (2012). Application of coarse-grid computational fluid dynamics on indoor environment modeling: Optimizing the trade-off between grid resolution and simulation accuracy. HVAC\&R Research, 18(5), 915-933.

[76] Wang, H., Zhai, Z. J., \& Liu, X. (2014, April). Feasibility of utilizing numerical viscosity from coarse grid CFD for fast turbulence modeling of indoor environments. In Building Simulation (Vol. 7, No. 2, pp. 155-164). Tsinghua University Press.

[77] Wilcox, D. C. (1998). Turbulence modeling for CFD (Vol. 2, pp. 172-180). La Canada, CA: DCW industries.

[78] Willcox, K., \& Megretski, A. (2005). Fourier series for accurate, stable, reducedorder models in large-scale linear applications. SIAM Journal on Scientific Computing, 26(3), 944-962.

[79] Yakhot, V., \& Orszag, S. A. (1986). Renormalization group analysis of turbulence. I. Basic theory. Journal of scientific computing, 1(1), 3-51.

[80] Yuan, T., Cizmas, P. G., \& O’Brien, T. (2005). A reduced-order model for a bubbling fluidized bed based on proper orthogonal decomposition. Computers \& chemical engineering, 30(2), 243-259.

[81] Zhai, J. Z., Hermansen, K. A., \& Al-Saadi, S. (2012). The Development of Simplified Rack Boundary Conditions for Numerical Data Center Models. ASHRAE Transactions, 118(2).

[82] Zhai, Z. and Chen, Q. (2003). Solution characters of iterative coupling between energy simulation and CFD programs. Energy and Buildings, 25(5), pp. 493-505. 
[83] Zhai, Z. and Chen, Q. (2005). Performance of coupled building energy and CFD simulations. Energy and Buildings 37, pp. $333-344$.

[84] Zhai, Z. and Chen, Q. (2006). Sensitivity analysis and applications guides for integrated building energy and CFD simulation. Energy and Building 38, pp. 1060 1068.

[85] Zhai, Z. and Chen, Q. Strategies for coupling energy simulation and computational fluid dynamics programs. Seventh International IBPSA Conference, Rio de Janeiro, Brazil, pp 59-66, 2001.

[86] Zhang, X., VanGilder, J. W., Iyengar, M., \& Schmidt, R. R. (2008, May). Effect of rack modeling detail on the numerical results of a data center test cell. In 2008 11th Intersociety Conference on Thermal and Thermomechanical Phenomena in Electronic Systems (pp. 1183-1190). IEEE.

[87] Zhang, Z., Zhang, W., Zhai, Z. J., \& Chen, Q. Y. (2007). Evaluation of various turbulence models in predicting airflow and turbulence in enclosed environments by CFD: Part 2 - Comparison with experimental data from literature. Hvac\&R Research, 13(6), 871-886.

[88] Zuo, W., \& Chen, Q. (2010). Fast and informative flow simulations in a building by using fast fluid dynamics model on graphics processing unit. Building and environment, 45(3), 747-757. 


\section{Appendix A}

Table A.1 Turbulence model governing equations and model constants

\begin{tabular}{|c|c|c|}
\hline Turbulence Models & Transport Equation (s) & Terms and Model Constant(s) \\
\hline 0-equation (Chen $-\mathrm{Xu})$ & & $\mu_{t}=0.03874 \rho v l$ \\
\hline $\begin{array}{c}\text { 1-equation (Spalart - } \\
\text { Allmaras) }\end{array}$ & $\begin{aligned} \frac{\partial}{\partial t}(\rho \widetilde{v})+\frac{\partial}{\partial x_{i}}\left(\rho \widetilde{v} u_{i}\right)=G_{v} & +\frac{1}{\sigma_{\widetilde{v}}}\left[\frac{\partial}{\partial x_{j}}\left\{(\mu+\rho \widetilde{v}) \frac{\partial \widetilde{v}}{\partial x_{j}}\right\}\right. \\
& \left.+C_{b 2} \rho\left(\frac{\partial \widetilde{v}}{\partial x_{j}}\right)^{2}\right]-Y_{v}+S_{\widetilde{v}}\end{aligned}$ & $\begin{aligned} & \mu_{t}=\rho \widetilde{v} f_{v 1} ; G_{v}=C_{b 1} \rho\left[\sqrt{2 \Omega_{i j} \Omega_{i j}}+\frac{\widetilde{v}}{k^{2} d^{2}} f_{v 2}\right], \text { where } f_{v 2}=1-\frac{X}{1+X f_{v 1}}, f_{v 1}=\left[\frac{X^{3}}{X^{3}+C_{v 1}^{3}}\right], X \equiv \frac{\widetilde{v}}{v} \\
& Y_{v}=C_{w 1} \rho f_{w}\left(\frac{v}{d}\right)^{2}, \text { where } f_{w}=g\left[\frac{1+c_{w 3}^{6}}{g^{6}+C_{w 3}}\right]^{\frac{1}{6}}, g=r+C_{w 2}\left(r^{6}-r\right), r \equiv \frac{v}{\bar{S}_{k^{2} d^{2}}} \\
& C_{b 1}=0.1355 ; C_{b 2}=0.622 ; \sigma_{\tilde{v}}=\frac{2}{3} ; C_{v 1}=7.1 ; C_{w 1}=\frac{C_{b 1}}{k^{2}}+\frac{\left(1+C_{b 2}\right)}{\sigma_{\tilde{v}}} ; C_{w 2}=0.3 ; C_{w 3}=2.0 ; \kappa=0.4187\end{aligned}$ \\
\hline $\begin{array}{l}\text { Standard } k-\varepsilon \text { (Launder } \\
\text { and Spalding) }\end{array}$ & $\begin{array}{c}\frac{\partial}{\partial t}(\rho k)+\frac{\partial}{\partial x_{i}}\left(\rho k u_{i}\right)=\frac{\partial}{\partial x_{j}}\left[\left(\mu+\frac{\mu_{t}}{\sigma_{k}}\right) \frac{\partial k}{\partial x_{j}}\right]+G_{k}+G_{b}-\rho \varepsilon \\
\frac{\partial}{\partial t}(\rho \varepsilon)+\frac{\partial}{\partial x_{i}}\left(\rho \varepsilon u_{i}\right)=\frac{\partial}{\partial x_{j}}\left[\left(\mu+\frac{\mu_{t}}{\sigma_{\varepsilon}}\right) \frac{\partial \varepsilon}{\partial x_{j}}\right]+C_{1 \varepsilon} \frac{\varepsilon}{k}\left(G_{k}+C_{3 \varepsilon} G_{b}\right) \\
-C_{2 \varepsilon} \rho \frac{\varepsilon^{2}}{k}+S_{\varepsilon}\end{array}$ & $\begin{array}{c}\mu_{t}=\rho C_{\mu} \frac{k^{2}}{\varepsilon} ; G_{k}=-\rho \overline{u_{u}^{\prime} u_{j}^{\prime}} \frac{\partial u_{j}}{\partial x_{i}} ; G_{b}=\beta g_{i} \frac{\mu_{t}}{P r_{t}} \frac{\partial T}{\partial X_{i}}, \text { where } \beta=-\frac{1}{\rho}\left(\frac{\partial \rho}{\partial T}\right)_{p} ; S_{k} \& S_{\varepsilon}=\text { user source terms; } \\
Y_{M}=2 \rho \varepsilon M_{t}^{2}, \text { where } M_{t}^{2}=\sqrt{\frac{k}{a^{2}}}, a \equiv \sqrt{\gamma R T} \\
C_{1 \varepsilon}=1.44 ; C_{2 \varepsilon}=1.92 ; C_{\mu}=0.09 ; \sigma_{k}=1.0 ; \sigma_{\varepsilon}=1.3\end{array}$ \\
\hline $\begin{array}{c}\text { RNG } k-\varepsilon \text { (Yakhot and } \\
\text { Orszag) }\end{array}$ & $\begin{array}{c}\frac{\partial}{\partial t}(\rho k)+\frac{\partial}{\partial x_{i}}\left(\rho k u_{i}\right)=\frac{\partial}{\partial x_{j}}\left[\alpha_{k} \mu_{e f f} \frac{\partial k}{\partial x_{j}}\right]+G_{k}+G_{b}-\rho \varepsilon-Y_{M} \\
+S_{k} \\
\frac{\partial}{\partial t}(\rho \varepsilon)+\frac{\partial}{\partial x_{i}}\left(\rho \varepsilon u_{i}\right)=\frac{\partial}{\partial x_{j}}\left[\alpha_{\varepsilon} \mu_{e f f} \frac{\partial \varepsilon}{\partial x_{j}}\right]+C_{1 \varepsilon} \frac{\varepsilon}{k}\left(G_{k}+C_{3 \varepsilon} G_{b}\right) \\
-C_{2 \varepsilon} \rho \frac{\varepsilon^{2}}{k}-R_{\varepsilon}+S_{\varepsilon}\end{array}$ & $\begin{array}{c}d\left(\frac{\rho^{2} k}{\sqrt{\varepsilon \mu}}\right)=1.72 \frac{\widehat{v}}{\sqrt{\hat{v}^{3}-1+C_{v}}} d \widehat{v}, \text { where } \widehat{v}=\frac{\mu_{e f f}}{\mu}, C_{v} \approx 100 ; G_{k}, G_{b}, Y_{M}=\text { same as standard } k-\varepsilon ; \\
S_{k} \& S_{\varepsilon}=\text { user source terms } ; R_{\varepsilon}=\frac{\left.C_{\mu \rho \eta^{3}\left(1-\frac{\eta}{\eta_{0}}\right)}\right)}{1+\beta \eta^{3}}, \frac{\varepsilon^{3}}{k} \text {, where } \eta \equiv \frac{s k}{\varepsilon}, \eta_{0}=4.38, \beta=0.012 \\
\alpha_{k}=\alpha_{\varepsilon}=1.393 ; C_{1 \varepsilon}=1.42 ; C_{2 \varepsilon}=1.68\end{array}$ \\
\hline $\begin{array}{l}\text { Realizable } k-\varepsilon \text { (Shih et } \\
\text { al.) }\end{array}$ & $\begin{array}{c}\frac{\partial}{\partial t}(\rho k)+\frac{\partial}{\partial x_{i}}\left(\rho k u_{i}\right)=\frac{\partial}{\partial x_{j}}\left[\left(\mu+\frac{\mu_{t}}{\sigma_{k}}\right) \frac{\partial k}{\partial x_{j}}\right]+G_{k}+G_{b}-\rho \varepsilon \\
\frac{\partial}{\partial t}(\rho \varepsilon)+\frac{\partial}{\partial x_{i}}\left(\rho \varepsilon u_{i}\right)=\frac{\partial}{\partial x_{j}}\left[\left(\mu+\frac{\mu_{t}}{\sigma_{\varepsilon}}\right) \frac{\partial \varepsilon}{\partial x_{x}}\right]+\rho C_{1} s \varepsilon \\
-\rho C_{2} \frac{\varepsilon^{2}}{k+\sqrt{v \varepsilon}}+C_{1 \varepsilon} \frac{\varepsilon}{k} C_{3 \varepsilon} G_{b}+S_{\varepsilon}\end{array}$ & 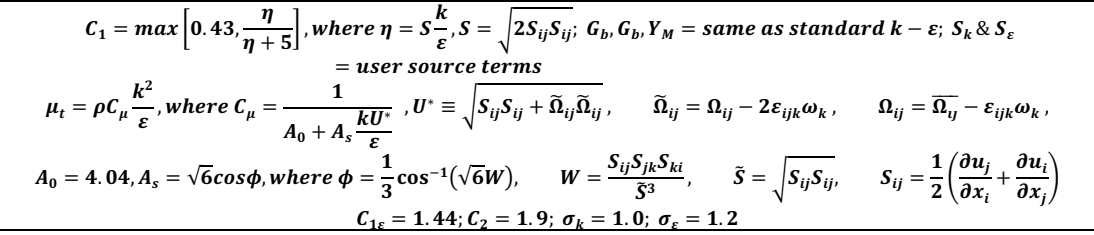 \\
\hline $\begin{array}{c}\text { LRN k- } \varepsilon \text { (Launder and } \\
\text { Sharma) }\end{array}$ & $\begin{array}{c}\frac{\partial}{\partial t}(\rho k)+\frac{\partial}{\partial i}\left(\rho k u_{i}\right)=\frac{\partial}{\partial x_{j}}\left[\left(\mu+\frac{\mu_{t}}{\sigma_{k}}\right) \frac{\partial k}{\partial x_{j}}\right]+G_{k}+G_{b}-\rho \varepsilon-Y_{M} \\
+D+S_{k} \\
\frac{\partial}{\partial t}(\rho \varepsilon)+\frac{\partial}{\partial x_{i}}\left(\rho \varepsilon u_{i}\right)=\frac{\partial}{\partial x_{j}}\left[\left(\mu+\frac{\mu_{t}}{\sigma_{\varepsilon}}\right) \frac{\partial \varepsilon}{\partial x_{j}}\right]+\frac{C_{\varepsilon 1}^{*} G_{k} \varepsilon}{k}-\frac{C_{\varepsilon 2}^{*} \rho \varepsilon^{2}}{k} \\
+E+S_{\varepsilon}\end{array}$ & $\begin{array}{c}\mu_{t}=\frac{f_{\mu} C_{\mu} \rho k^{2}}{\varepsilon} ; f_{\mu}=\exp \left[-\frac{3.4}{\left(1+\frac{R e_{t}}{50}\right)^{2}}\right] ; D=2 \mu_{t}\left(\frac{\partial k^{\frac{1}{2}}}{\partial x_{\perp}}\right) \\
E=\frac{2 \mu \mu_{t}}{\rho}\left(\frac{\partial^{2} u_{/ /}}{\partial^{2} x_{\perp}}\right) ; C_{\varepsilon 1}^{*}=C_{\varepsilon 1} ; C_{\varepsilon 2}^{*}=C_{\varepsilon 2}=\left[1-0.3 \exp \left(-R e_{t}^{2}\right)\right]\end{array}$ \\
\hline LRN k- $\omega$ (Wilcox) & $\begin{array}{l}\frac{\partial}{\partial t}(\rho k)+\frac{\partial}{\partial x_{i}}\left(\rho k u_{i}\right)=\frac{\partial}{\partial x_{j}}\left[\Gamma_{k} \frac{\partial k}{\partial x_{j}}\right]+G_{k}-Y_{k}+S_{k} \\
\frac{\partial}{\partial t}(\rho \omega)+\frac{\partial}{\partial x_{i}}\left(\rho \omega u_{i}\right)=\frac{\partial}{\partial x_{j}}\left[\Gamma_{\omega} \frac{\partial \omega}{\partial x_{j}}\right]+G_{\omega}-Y_{\omega}+S_{\omega}\end{array}$ & $\begin{array}{c}\Gamma_{k}=\mu+\frac{\mu_{t}}{\sigma_{k}} ; \Gamma_{\omega}=\mu+\frac{\mu_{t}}{\sigma_{\omega}} ; \mu_{t}=\alpha^{*} \frac{\rho k}{\omega}, \text { where } \alpha^{*}=\alpha_{\infty}^{*}\left(\frac{\alpha_{0}^{*}+\frac{R e_{t}}{R_{k}}}{1+\frac{R e_{t}}{R_{k}}}\right), R e_{t}=\frac{\rho k}{\mu \omega}, R_{k}=6, \alpha_{0}^{*}=\frac{\beta_{i}}{3}, \beta_{i}=0.072, \alpha^{*}=\alpha_{\infty}^{*} \\
=1 \\
G_{k}=-\rho \overline{u_{i}^{\prime} u_{j}^{\prime}} \frac{\partial u_{j}}{\partial x_{i}} ; G_{\omega}=\alpha \frac{\omega}{k} G_{k}, \text { where } \alpha=\frac{\alpha_{\infty}}{\alpha^{*}}\left(\frac{\alpha_{0}+\frac{R e_{t}}{R_{\omega}}}{1+\frac{R e_{t}}{R_{\omega}}}\right), R_{\omega}=2.95 ; Y_{k}=\rho \beta^{*} f_{\beta^{*}} k \omega ; Y_{\omega}=\rho \beta f_{\beta} \omega^{2} \\
\begin{aligned} \alpha_{\infty}^{*}=1 ; \alpha_{\infty}=0.52 ; \alpha_{0}=\frac{1}{9} ; \beta_{\infty}^{*}=0.09 ; \beta_{i}=0.072 ; R_{\beta}=8 ; R_{k}=6 ; R_{\omega}=2.95 ; \zeta^{*}=1.5 ; M_{t 0}=0.25 ; \sigma_{k}=2.0 ; \sigma_{\omega} \\
=2.0\end{aligned}\end{array}$ \\
\hline
\end{tabular}


(Table A.1 Continued)

\begin{tabular}{|c|c|c|}
\hline SST $k$ - $\omega$ (Menter) & $\begin{array}{c}\frac{\partial}{\partial t}(\rho k)+\frac{\partial}{\partial x_{i}}\left(\rho k u_{i}\right)=\frac{\partial}{\partial x_{j}}\left[\Gamma_{\frac{1}{k}} \frac{\partial k}{\partial x_{j}}\right]+G_{k}-Y_{k}+S_{k} \\
\frac{\partial}{\partial t}(\rho \omega)+\frac{\partial}{\partial x_{i}}\left(\rho \omega u_{i}\right)=\frac{\partial}{\partial x_{j}}\left[\Gamma_{\omega} \frac{\partial \omega}{\partial x_{j}}\right]+G_{\omega}-Y_{\omega}+D_{\omega}+S_{\omega}\end{array}$ & 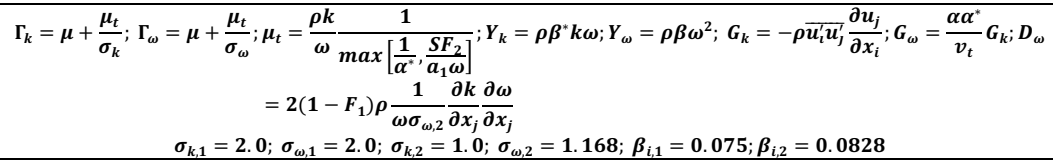 \\
\hline V2f (Durbin) & $\begin{array}{c}\frac{\partial}{\partial t}(\rho k)+\frac{\partial}{\partial x_{i}}\left(\rho k u_{i}\right)=P-\rho \varepsilon+\frac{\partial}{\partial x_{j}}\left[\left(\mu+\frac{\mu_{t}}{\sigma_{k}}\right) \frac{\partial k}{\partial x_{j}}\right]+S_{k} \\
\frac{\partial}{\partial t}(\rho \varepsilon)+\frac{\partial}{\partial x_{i}}\left(\rho \varepsilon u_{i}\right)=\frac{C_{\varepsilon 1}^{\prime} P-C_{2 \varepsilon} \rho \varepsilon}{T}+\frac{\partial}{\partial x_{j}}\left[\left(\mu+\frac{\mu_{t}}{\sigma_{\varepsilon}}\right) \frac{\partial \varepsilon}{\partial x_{j}}\right]+S_{\varepsilon} \\
\frac{\partial}{\partial t}\left(\rho \overline{v^{2}}\right)+\frac{\partial}{\partial x_{i}}\left(\rho \overline{v^{2}} u_{i}\right)=\rho k f-6 \overline{v^{2}} \frac{\varepsilon}{k}+\frac{\partial}{\partial x_{j}}\left[\left(\mu+\frac{\mu_{t}}{\sigma_{k}}\right) \frac{\partial \bar{v}^{2}}{\partial x_{j}}\right] \\
\quad+S_{\overline{v^{2}}} \\
f-L^{2} \frac{\partial^{2} f}{\partial x_{j}^{2}}=\left(C_{1}-1\right) \frac{\frac{2}{3}-\frac{\bar{v}^{2}}{k}}{T}+C_{2} \frac{P}{\rho k}+\frac{\frac{5 v^{2}}{k}}{T}+S_{f}\end{array}$ & 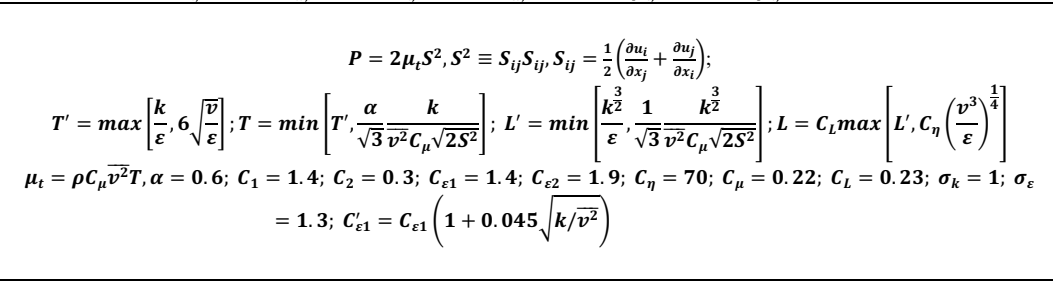 \\
\hline $\begin{array}{l}\text { RSM (Gibson and } \\
\text { Launder) }\end{array}$ & 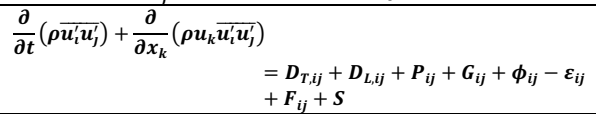 & 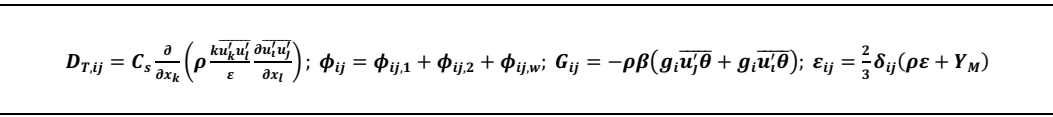 \\
\hline
\end{tabular}




\section{Appendix B}

\section{Linear tile airflow distribution}

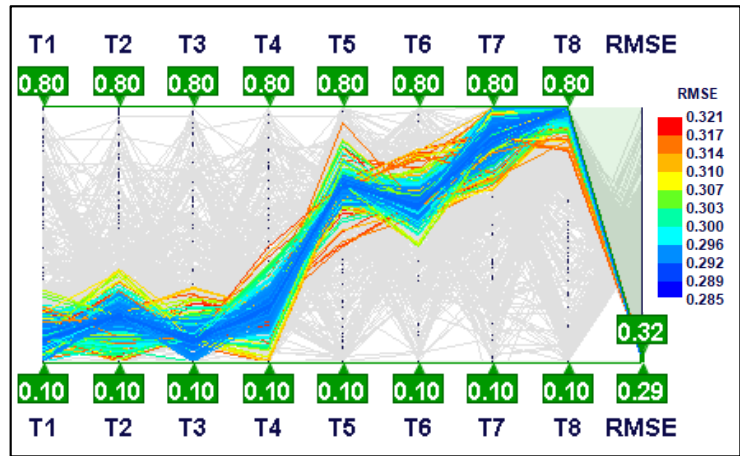

a) Stage 1

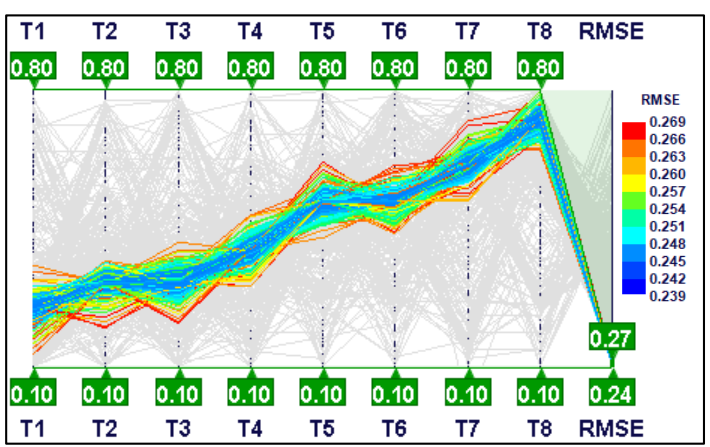

c) Stage 3

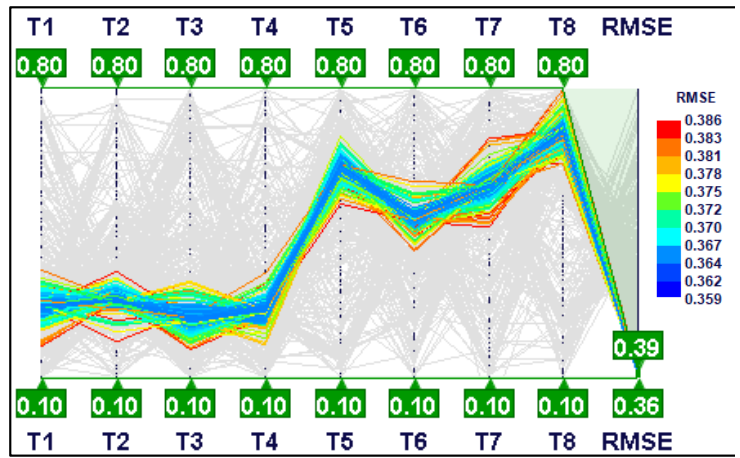

b) Stage 2

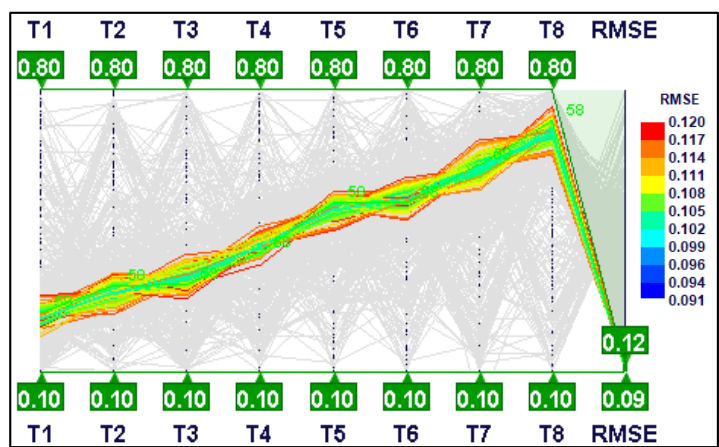

d) Stage 4

Fig. B.1 Optimal designs sort out at different stages 


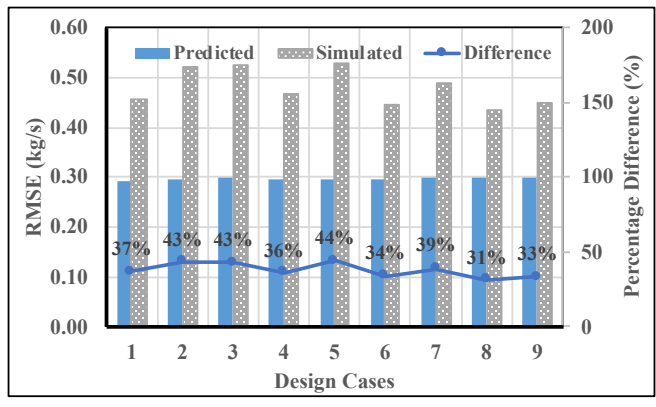

a) Stage 1

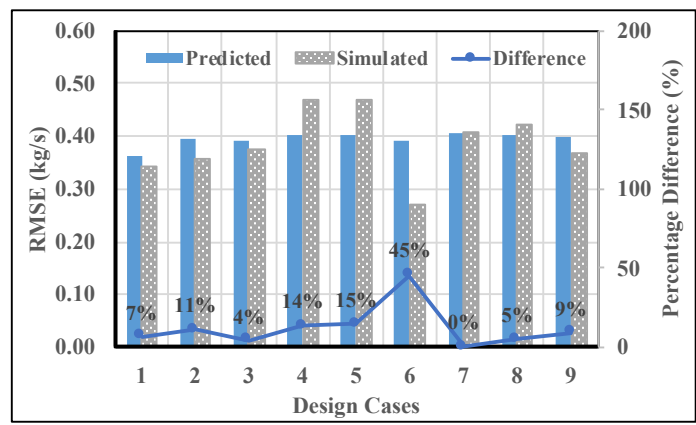

c) Stage 2

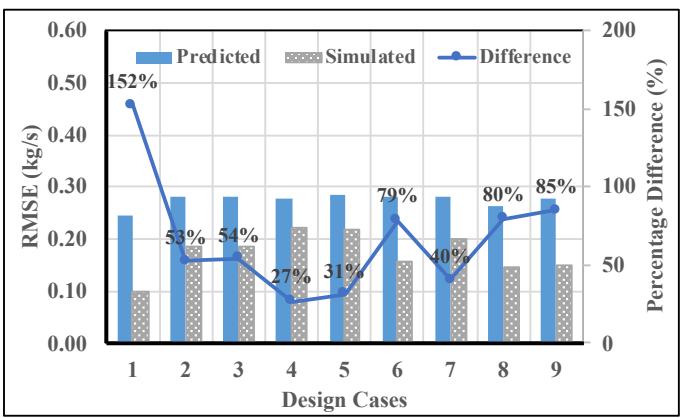

e) Stage 3

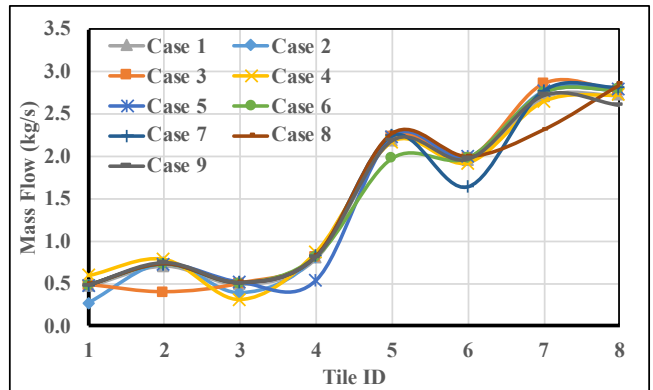

b) Stage 1

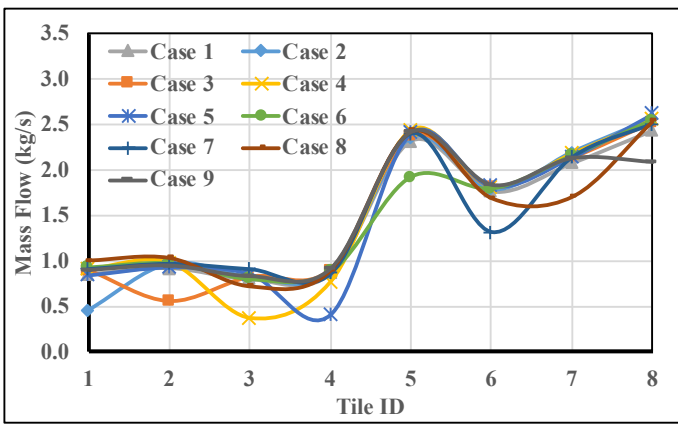

d) Stage 2

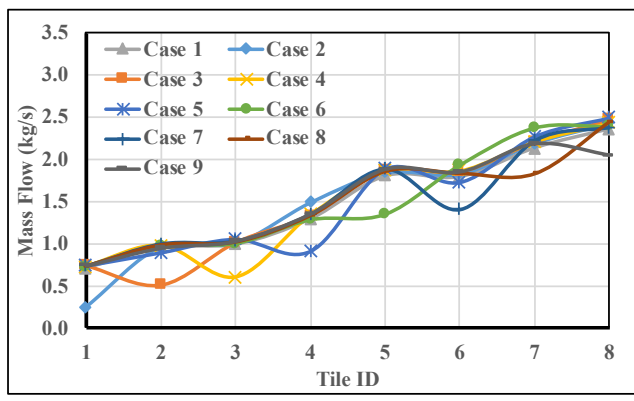

f) Stage 3

Fig. B.2 Designs validation via stages

a), c), e) Comparison of the tile mass flow rate standard deviation between the predicted RSM and the simulated CFD values. b), d), f) The simulated CFD values of tile mass flow rate for cases validated at each stage 


\section{Parabolic tile airflow distribution}

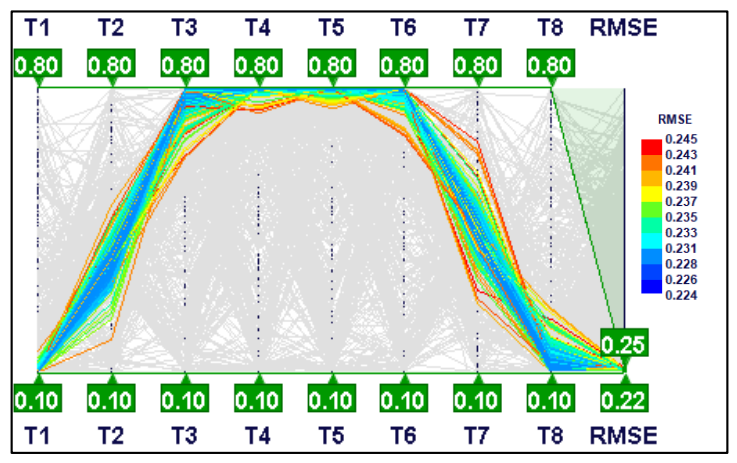

a) Stage 1

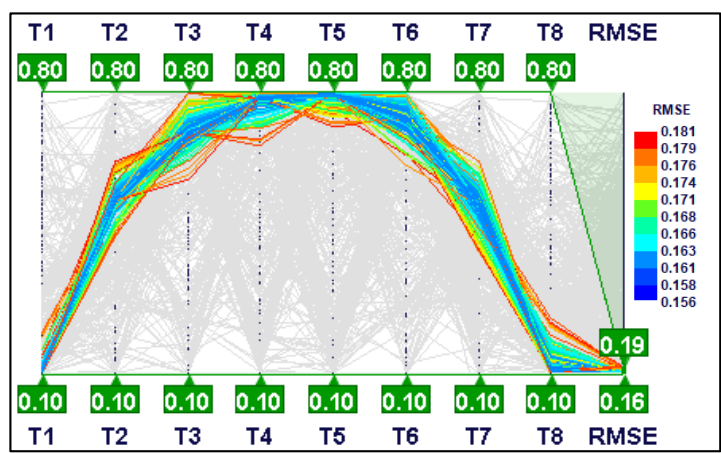

c) Stage 3

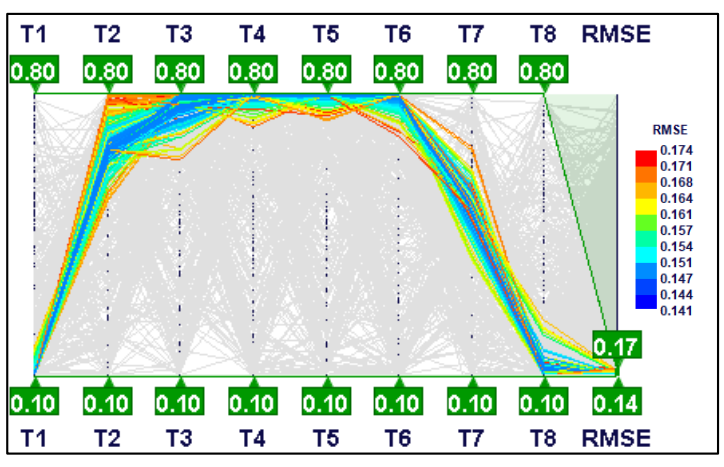

b) Stage 2

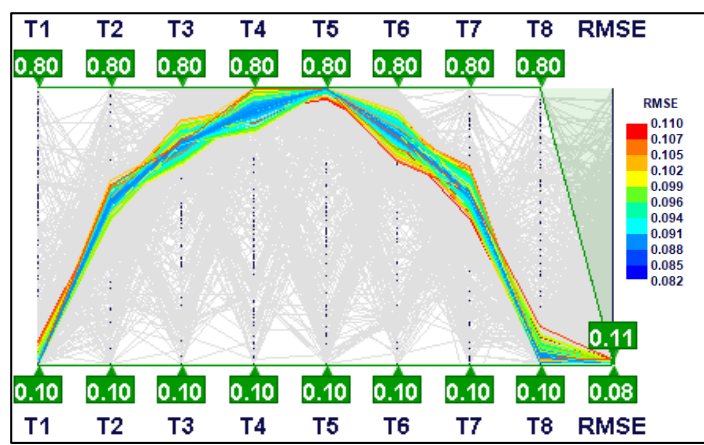

d) Stage 4

Fig. B.3 Optimal designs sort out at different stages 


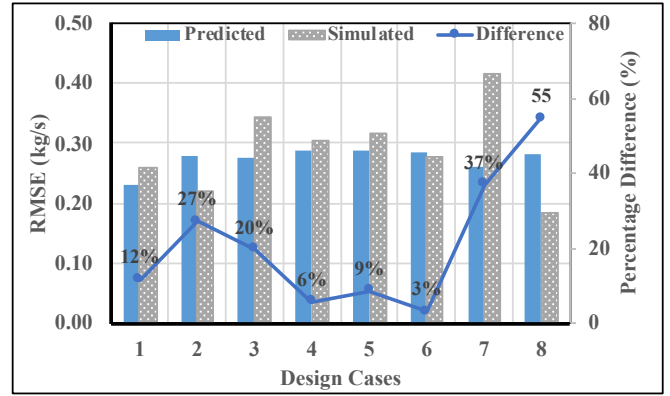

a) Stage 1

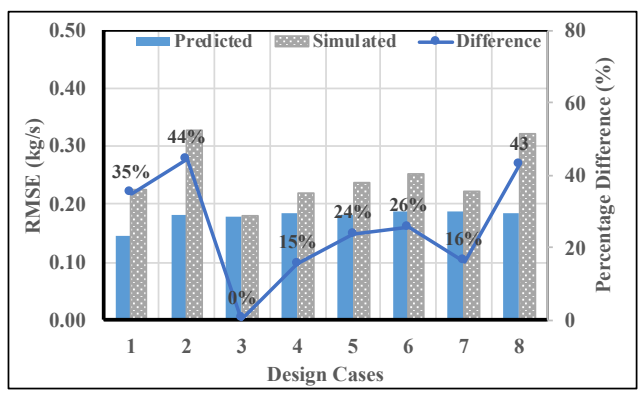

c) Stage 2

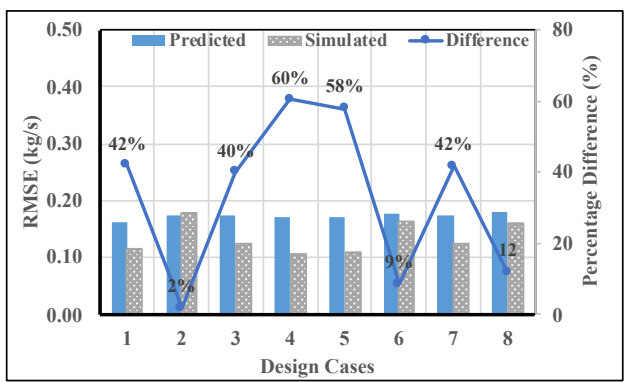

e) Stage 3

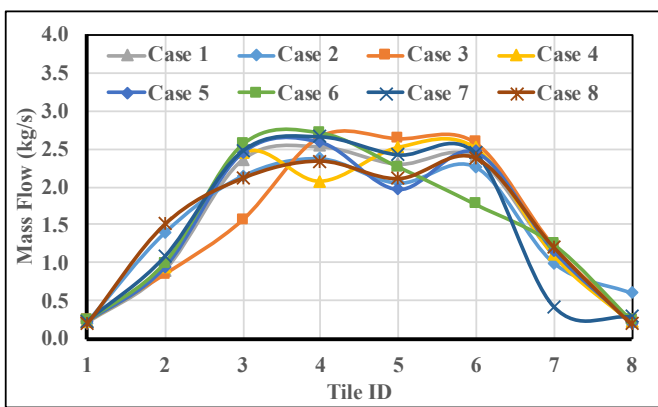

b) Stage 1

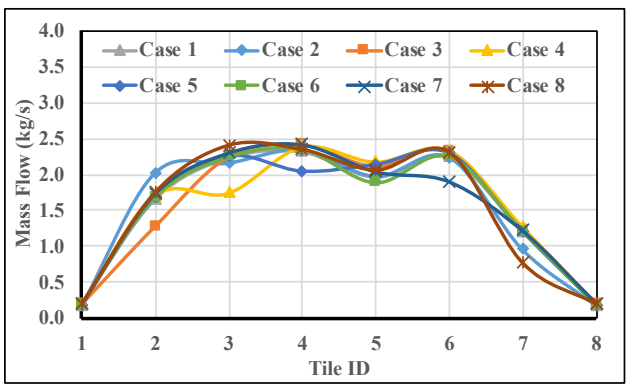

d) Stage 2

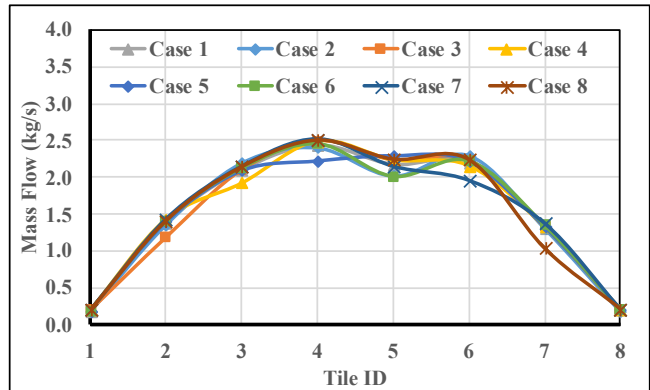

f) Stage 3

Fig. B.4 Design validation via stages ge

a), c), e) Comparison of the tile mass flow rate standard deviation between the predicted RSM and the simulated CFD values. b), d), f) The simulated CFD values of tile mass flow rate for cases validated at each stage 


\section{Sinusoidal tile airflow distribution}

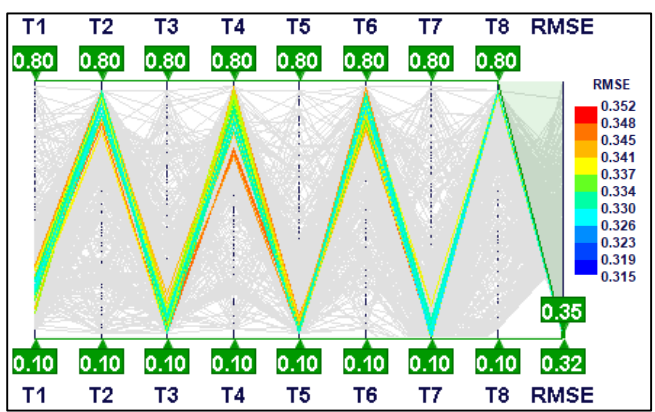

a) Stage 1

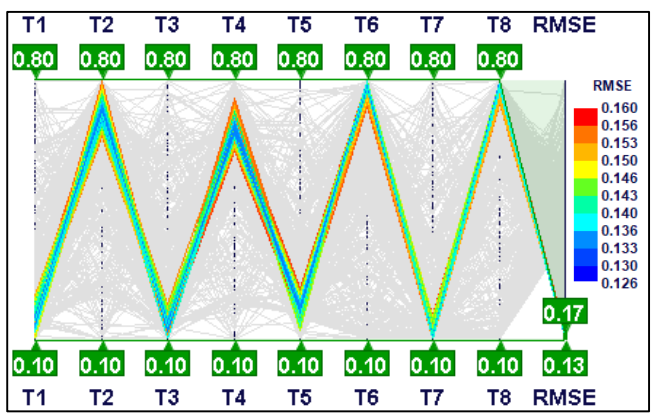

c) Stage 3

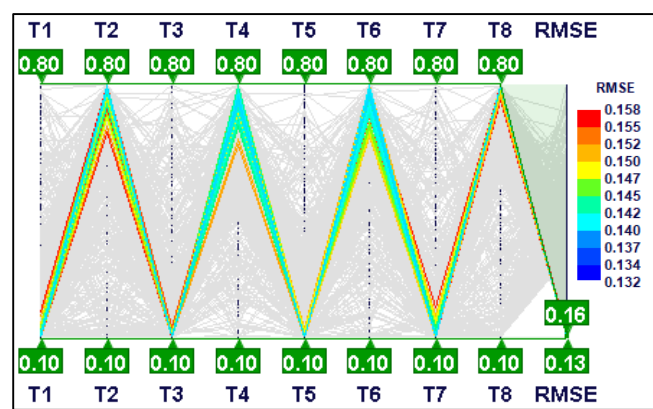

b) Stage 2

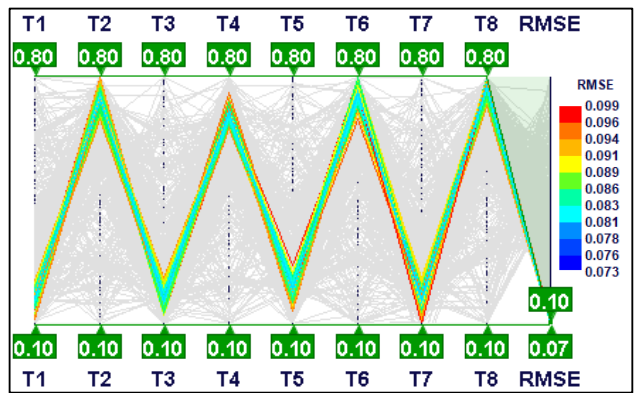

d) Stage 4

Fig. B.5 Optimal designs sort out at different stages 


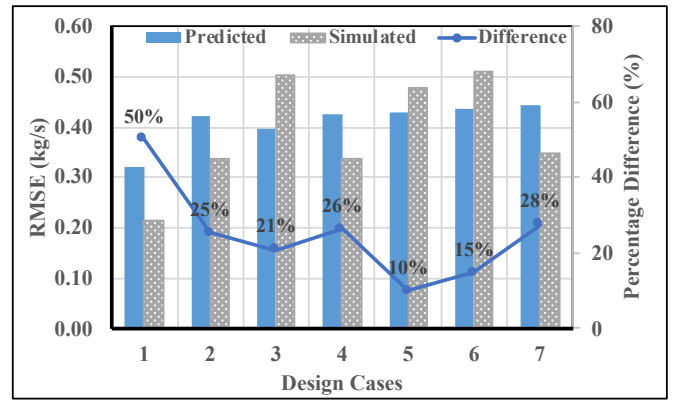

a) Stage 1

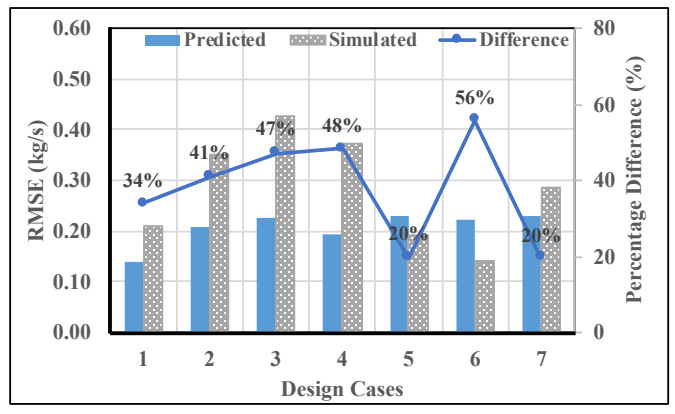

c) Stage 2

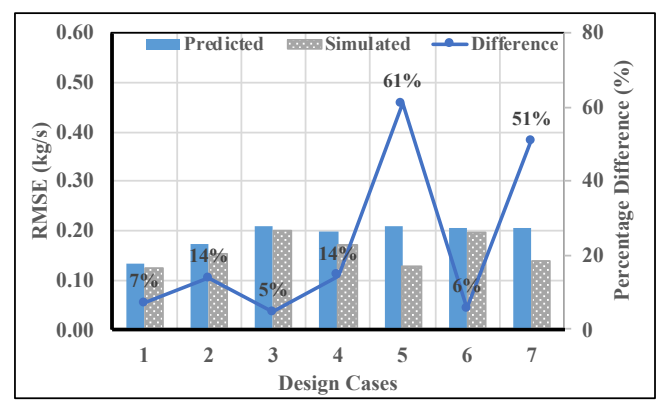

e) Stage 3

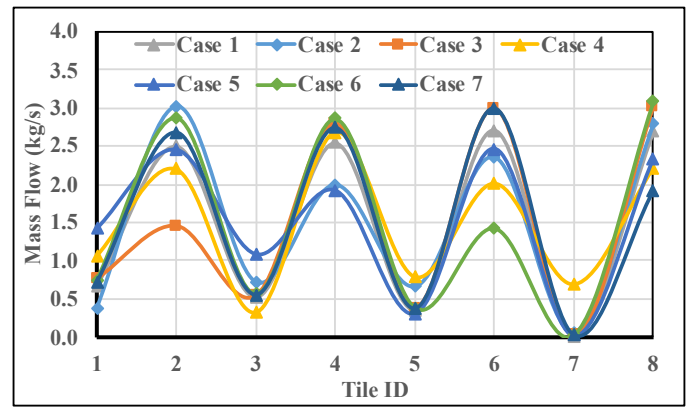

b) Stage 1

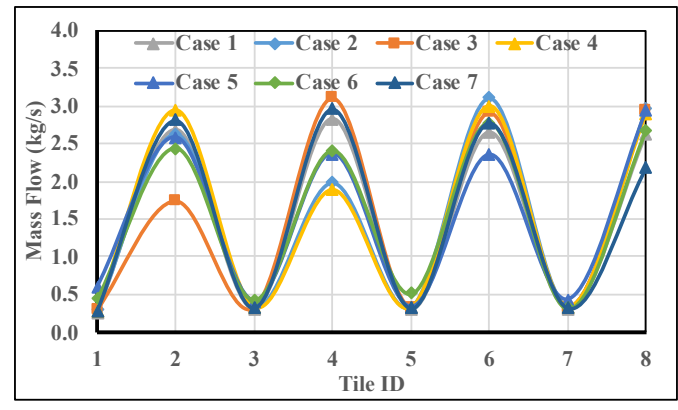

d) Stage 2

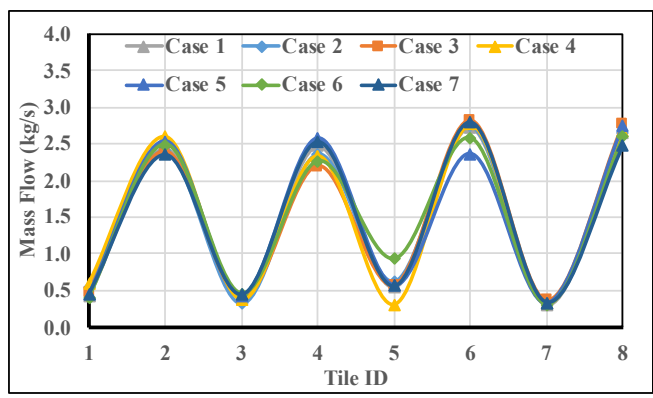

f) Stage 3

Fig. B.6 Design validation via stages

a), c), e) Comparison of the tile mass flow rate standard deviation between the predicted RSM and the simulated CFD values. b), d), f) The simulated CFD values of tile mass flow rate for cases validated at each stage 
VITA

\section{LONG PHAN}

Born, Laem Sing, Chanthaburi, Thailand

$2011-2013$

B.S., Mechanical Engineering

Florida International University

Miami, Florida

$2013-2015$

M.S., Mechanical Engineering

Florida International University

Miami, Florida

$2015-2019$

Doctoral Candidate

Florida International University

Miami, Florida

\section{PUBLICATIONS \& PRESENTATIONS}

Phan, L., \& Lin, C. X. (2019). CFD-based response surface methodology for rapid thermal simulation and optimal design of data centers. Advances in Building Energy Research, pp.1 -23 .

Phan, L., Hu, B., \& Lin, C. X. (May 2019). An evaluation of turbulence \& tile models at server rack level for data centers. Journal of Building \& Environment, Vol. 155., pp. 421 -435 .

Phan, L., \& Lin, C. X. (Jul 2014). A multi-zone building simulation of a data center model with hot \& cold aisles. Journal of Energy \& Buildings. Vol. 77, p. 364 - 376.

Phan, L., \& Lin, C.X. (Feb 2017). Reduced order modeling of a data center model with multi-parameters. Journal of Energy \& Buildings, Vol. 136, p. 86 - 99.

Lin, C. X., \& Phan, L. (Apr 2013). A numerical study of both internal and external twophase flows of a rotating disk atomizer. Drying technology, 31(5), 605-613.

Dunkelberg, H., Wagner, J., Hannen, C., Schlüter, B. A., Phan, L., Hesselbach, J., \& Lin, C. X. (Aug 2018). Optimization of the energy supply in the plastics industry to reduce the primary energy demand. Journal of Cleaner Production. Vol. 192, p. $790-800$.

Phan, L., Soleimanikutanei, S., Lee, E.J., \& Lin, C.X. CFD model validation of the turbulent flows in inserted pipes for a ground-air heat exchanger application. 3rd Thermal \& Fluids Engineering Conference. Fort Lauderdale, FL, March 4 - 7, 2018. 
Phan, L., Soleimanikutanei, S., Lee, E.J., \& Lin, C.X. CFD model validation of the turbulent flows in inserted pipes for a ground-air heat exchanger application. 3rd Thermal \& Fluids Engineering Conference. Fort Lauderdale, FL, March 4 - 7, 2018.

Phan, L., Bhusal, S., \& Lin, C.X. Improving cooling efficiency by using mixed tiles to control airflow uniformity of perforated tiles in a data center model. ASME 2017 Summer Heat Transfer Conference. Bellevue, WA, Jul 9 - 14, 2017.

Phan, L., Liu, B., \& Lin, C.X. (2016, June). A hybrid turbulence model coupling strategy for cfd simulation of a data center model. ASHRAE Annual Conference, St. Louis, MO, USA, ST-16-C005.

Phan, L., Telusma, M., \& Lin, C.X. Data center modeling using response surface with multi-parameters approach. ASME International Mechanical Congress \& Exposition. Houston, TX, Nov $13-19,2015$.

Phan, L., \& Lin, C. X. A parametric study of temperature \& volumetric flow rate parameters of a data center using multi-zone building simulation. 2013 ASME International Mechanical Engineering Congress \& Exposition. San Diego, CA. Nov 15 21, 2013.

Phan, L., \& Lin, C.X. A numerical investigation of internal \& external two-phase flows of a rotating disk atomizer. ASME Fluids Engineering Division Summer Meeting. Puerto Rico, USA, Jul 8 - 12, 2012.

Phan, L., \& Lin, C.X. Optimization study for stanchion layout \& flow partitioning to archive uniform airflow through perforated tiles in data centers. ASHRAE Winter Conference in Chicago, Jan $22-24,2018$.

Phan, L., \& Lin, C.X. Evaluation of strategies for uniform airflow through perforated tiles in data center. ASHRAE Winter Conference in Las Vegas, Jan 28 - Feb 1, 2017.

Phan, L., \& Lin, C.X. Recent advances in fast modeling of data centers. ASME 2016 Summer Heat Transfer Conference in Capitol Hill, Washington DC, Jul 10 - 14, 2016.

Phan, L., \& Lin, C.X. Data center modeling using response surface methodology. ASHRAE Annual Conference. St. Louis, June. 2016.

Phan, L., \& Lin, C.X. Reduced order modeling (rom) of airflow \& thermal fields in a data center. ASHRAE Winter Conference in Orlando, Jan 23-27, 2016.

Phan, L., \& Lin, C.X. FIU Solar House's Potential Performance: A study of natural ventilation strategies. Second Annual Go Solar \& Renewable Energy Fest in Fort Lauderdale, FL, Jun 6-7, 2014. 